\title{
On the Stability of Periodic Multi-Solitons of the KdV Equation
}

\author{
Thomas Kappeler $^{1}{ }^{\mathbb{D}}$, Riccardo Montalto ${ }^{2}$ \\ ${ }^{1}$ Institut für Mathematik, Universität Zürich, Winterthurerstrasse 190, 8057 Zürich, Switzerland. \\ E-mail: thomas.kappeler@math.uzh.ch \\ 2 University of Milan, Via Saldini 50, 20133 Milan, Italy. \\ E-mail: riccardo.montalto@unimi.it
}

Received: 9 September 2020 / Accepted: 29 March 2021

Published online: 11 May 2021 - (C) The Author(s) 2021

\begin{abstract}
In this paper we obtain the following stability result for periodic multisolitons of the $\mathrm{KdV}$ equation: We prove that under any given semilinear Hamiltonian perturbation of small size $\varepsilon>0$, a large class of periodic multi-solitons of the KdV equation, including ones of large amplitude, are orbitally stable for a time interval of length at least $O\left(\varepsilon^{-2}\right)$. To the best of our knowledge, this is the first stability result of such type for periodic multi-solitons of large size of an integrable PDE.
\end{abstract}

\section{Contents}

1. Introduction . . . . . . . . . . . . . . . . . . . . 1872

2. Para-Differential Calculus . . . . . . . . . . . . . . . . . . . 1888

3. Para-Differential Vector Fields . . . . . . . . . . . . . . . . . . . . . . . 1891

3.1 Definitions . . . . . . . . . . . . . . . . . . . . . . . . 1892

3.2 Commutators . . . . . . . . . . . . . . . . . . . . . . . . . 1894

3.3 Flows of para-differential vector fields . . . . . . . . . . . . . . . 1897

3.4 Flows of Fourier multiplier vector fields and smoothing vector fields . 1906

4. Reformulation of Theorem 1.1 and Normal Form Theorem . . . . . . . . 1912

5. Smoothing Normal Form Steps _. . . . . . . . . . . . . . . . . 1918

6. Normalization Steps by Para-Differential Calculus . . . . . . . . . . . 1925

6.1 Normalization of terms linear or quadratic in $w \ldots \ldots$. . . . . . . . 1925

6.2 Normalization of Fourier multiplier quadratic in $w \ldots \ldots$

6.3 Normalization of the smoothing remainders _ . . . . . . . . 1931

7. Proofs of Theorem 4.2 and Theorem $4.3 \ldots \ldots$. . . . . . . . . 1935

8. Measure Estimates . . . . . . . . . . . . . . . . . . . . . . . . . 1940

A. Linear Vector Fields on $H_{\perp}^{s}\left(\mathbb{T}_{1}\right) \ldots \ldots \ldots \ldots$

T. Kappeler: Supported in part by the Swiss National Science Foundation.

R. Montalto: Supported in part by the Swiss National Science Foundation and INDAM-GNFM. 
B. Standard Results on Homological Equations . . . . . . . . . . . . . . . . 1946

C. A Local Existence Result for $\partial_{t} \mathfrak{x}=X(\mathfrak{x}) \ldots \ldots$. . . . . . . . . . . . . . . . . 1946

D. On a Class of Linear Para-Differential Equations . . . . . . . . . . . . . . 1950

\section{Introduction}

The Korteweg-de Vries (KdV) equation

$$
\partial_{t} u=-\partial_{x}^{3} u+6 u \partial_{x} u
$$

is one of the most important model equations for describing dispersive phenomena. It is named after the two Dutch mathematician Korteweg and de Vries [29] (cf. also Boussinesq [14], Raleigh [40]) and originally was proposed as a model equation in one space dimension for long surface waves of water in a narrow and shallow channel. Today it is used in many branches of physics as well as in the engineering sciences. The seminal discovery in the late sixties that (1.1) admits infinitely many conservation laws $([34,38])$, and the development of the inverse scattering transform method ( [24]) led to the modern theory of integrable systems of finite and infinite dimension (see e.g. $[20,22]$, and references therein). More recently, as one of the most prominent examples among dispersive equations, (1.1) played a major role in the development of the theory of dispersive PDEs to which many of the leading analysts of our times contributed. In particular, the (globally in time) well-posedness theory of (1.1) has been established in various setups in great detail - see [19].

A distinguished feature of Eq. (1.1) is the existence of sharply localized traveling wave solutions of arbitrarily large amplitude and particle like properties. Kruskal and Zabusky, who studied them in numerical experiments in the early sixties (cf. [30]), coined the name 'soliton' for them. More generally, they found solutions, which are localized near finitely many points in space, referred to as multi-solitons. In the periodic setup, these solutions often are referred to as periodic multi-solitons or finite gap solutions. Due to their importance in applications, various stability aspects have been considered such as the long time asymptotics of solutions with initial data near (periodic) multisolitons (orbital stability, soliton resolution conjecture). Two major questions arise in connection with the structural stability of (1.1). One of them concerns the persistence of the (periodic) multi-solitons under perturbations of (1.1), and the other one concerns the long time asymptotics of solutions of perturbations of (1.1) with initial data close to a (periodic) multi-soliton. In the periodic setup, the first question has been studied quite extensively by developing KAM methods, pioneered by Kolmogorov, Arnold, and Moser to treat perturbations of finite dimensional integrable system, for PDEs (cf. [1,8, $12,15,28,31-33,35,39,41]$, and references therein), whereas the second one turned out to be quite challenging and little is known so far. Our goal is to address this longstanding open problem.

The aim of this paper is to study in the periodic setup the long time asymptotics of the solutions of Hamiltonian perturbations of (1.1) with initial data close to a periodic multisoliton of arbitrary large amplitude. To describe the class of perturbations considered, recall that (1.1) with the space periodic variable $x \in \mathbb{T}_{1}:=\mathbb{R} / \mathbb{Z}$ can be written in Hamiltonian form

$$
\partial_{t} u=\partial_{x} \nabla H^{k d v}(u), \quad H^{k d v}(u):=\int_{0}^{1}\left(\frac{1}{2}\left(\partial_{x} u\right)^{2}+u^{3}\right) d x,
$$


where $\nabla H^{k d v}(u)$ denotes the $L^{2}$-gradient of $H^{k d v}$ and where $\partial_{x}$ is the Poisson structure, corresponding to the Poisson bracket defined for functionals $F, G$ by

$$
\{F, G\}(u)=\int_{0}^{1} \nabla F \partial_{x} \nabla G d x .
$$

We consider semilinear Hamiltonian perturbations of (1.1) of the form

$$
\partial_{t} u=-\partial_{x}^{3} u+6 u \partial_{x} u+\varepsilon F(u)
$$

where $0<\varepsilon<1$ is a small parameter and $F$ is a semilinear Hamiltonian vector field

$$
F(u)=\partial_{x} \nabla P_{f}(u) .
$$

Here $P_{f}$ is a Hamiltonian of the form

$$
P_{f}(u):=\int_{0}^{1} f(x, u(x)) d x
$$

and $f$ a $C^{\infty}$-smooth density

$$
f: \mathbb{T}_{1} \times \mathbb{R} \rightarrow \mathbb{R}, \quad(x, \zeta) \mapsto f(x, \zeta),
$$

so that with $f^{\prime}(x, \zeta):=\partial_{\zeta} f(x, \zeta)$ and $f^{\prime \prime}(x, \zeta):=\partial_{\zeta}^{2} f(x, \zeta)$,

$$
F(u)(x)=\partial_{x} \nabla P_{f}(u)(x)=\partial_{x} f^{\prime}(x, u(x))+f^{\prime \prime}(x, u(x)) \partial_{x} u(x) .
$$

To state our main results, we first need to introduce some more notations. Since $u \mapsto\langle u\rangle_{x}:=\int_{0}^{1} u d x$ is a Casimir for the Poisson bracket (1.3) and hence a prime integral of (1.4), we restrict our attention to spaces of functions with zero mean (cf. [28], Section 13) and choose as phase spaces of (1.4) the scale of Sobolev spaces $H_{0}^{s}\left(\mathbb{T}_{1}\right)$, $s \in \mathbb{Z}_{\geq 0}$,

$$
H_{0}^{s}\left(\mathbb{T}_{1}\right):=\left\{q \in H^{s}\left(\mathbb{T}_{1}\right): \int_{0}^{1} q(x) d x=0\right\}, \quad L_{0}^{2}\left(\mathbb{T}_{1}\right) \equiv H_{0}^{0}\left(\mathbb{T}_{1}\right),
$$

where

$$
H^{s}\left(\mathbb{T}_{1}\right) \equiv H^{s}\left(\mathbb{T}_{1}, \mathbb{R}\right):=\left\{q=\sum_{n \in \mathbb{Z}} q_{n} e^{2 \pi i n x}: q_{n} \in \mathbb{C}, q_{-n}=\bar{q}_{n} \forall n \in \mathbb{Z},\|q\|_{s}<\infty\right\},
$$

and

$$
\|q\|_{s}=\left(\sum_{n \in \mathbb{Z}}\langle n\rangle^{2 s}\left|q_{n}\right|^{2}\right)^{\frac{1}{2}}, \quad\langle n\rangle:=\max \{1,|n|\}, \quad \forall n \in \mathbb{Z} .
$$

On $L_{0}^{2}\left(\mathbb{T}_{1}\right)$, the Poisson structure $\partial_{x}$ is nondegenerate and the corresponding symplectic form is given by

$$
\mathcal{W}_{L_{0}^{2}}(u, v):=\int_{0}^{1}\left(\partial_{x}^{-1} u\right) v d x, \quad \partial_{x}^{-1} u=\sum_{n \neq 0} \frac{1}{\mathrm{i} n} u_{n} e^{\mathrm{i} 2 \pi n x}, \quad \forall u, v \in L_{0}^{2}\left(\mathbb{T}_{1}\right)
$$


Note that the Hamiltonian vector field $X_{H}(u)=\partial_{x} \nabla H(u)$, associated with the Hamiltonian $H$, satisfies $d H(u)[\cdot]=\mathcal{W}_{L_{0}^{2}}\left(X_{H}, \cdot\right)$.

Our results can informally be stated as follows: for any $f \in C^{\infty}\left(\mathbb{T}_{1} \times \mathbb{R}\right), s$ sufficiently large, $\varepsilon>0$ sufficiently small, and for most of the finite gap solutions $q: t \mapsto q(t, \cdot)$ of (1.1), the following holds: for any initial data $u_{0} \in H_{0}^{s}\left(\mathbb{T}_{1}\right)$, which is $\varepsilon$-close in $H_{0}^{s}\left(\mathbb{T}_{1}\right)$ to the orbit $\mathcal{O}_{q}:=\{q(t, \cdot): t \in \mathbb{R}\}$ of $q$, the perturbed equation (1.4) admits a unique solution $t \mapsto u(t, \cdot)$ in $H_{0}^{s}\left(\mathbb{T}_{1}\right)$ with initial data $u(0, \cdot)=u_{0}$ and life span at least $[-T, T], T=O\left(\varepsilon^{-2}\right)$. The solution $u(t, \cdot)$ stays $\varepsilon$-close in $H_{0}^{s}\left(\mathbb{T}_{1}\right)$ to the orbit $\mathcal{O}_{q}$.

To state our results in precise terms, we need to define the notion of finite gap solution and the invariant tori, on which they evolve, and explain for which of these solutions the above stability results hold. Since these finite gap solutions are not small, we need to introduce coordinates to describe them. Most conveniently, this can be done in terms of a Euclidean version of action angle coordinates, referred to as Birkhoff coordinates. Let us now explain this in detail.

According to [28], the $\mathrm{KdV}$ equation (1.2) on $\mathbb{T}_{1}$ is an integrable PDE in the strongest possible sense, meaning that it admits globally defined canonical coordinates on $L_{0}^{2}\left(\mathbb{T}_{1}\right)$ so that when expressed in these coordinates, (1.2) can be solved by quadrature.

To describe these coordinates in more detail, we introduce for any $s \in \mathbb{Z}_{\geq 0}$ the weighted $\ell^{2}$-sequence spaces

$$
h_{0}^{s}:=\left\{\left(w_{n}\right)_{n \neq 0} \in h_{0, c}^{s}: w_{-n}=\bar{w}_{n} \forall n \geq 1\right\}, \quad \ell_{0}^{2} \equiv h_{0}^{0},
$$

where $h_{0, c}^{s} \equiv h^{s}(\mathbb{Z} \backslash\{0\}, \mathbb{C})$ is given by

$$
h_{0, c}^{s}:=\left\{w=\left(w_{n}\right)_{n \neq 0}: w_{n} \in \mathbb{C} \forall n \neq 0,\|w\|_{s}<\infty\right\}, \quad\|w\|_{s}:=\left(\sum_{n \neq 0}|n|^{2 s}\left|w_{n}\right|^{2}\right)^{\frac{1}{2}} .
$$

By [28] there exists a real analytic diffeomorphism, referred to as (complex) Birkhoff map,

$$
\Phi^{k d v}: L_{0}^{2}\left(\mathbb{T}_{1}\right) \rightarrow \ell_{0}^{2}, \quad q \mapsto w(q):=\left(w_{n}(q)\right)_{n \neq 0},
$$

which is canonical in the sense that

$$
\left\{w_{n}, w_{-n}\right\}=\int_{0}^{1} \nabla w_{n} \partial_{x} \nabla w_{-n} d x=2 \pi \mathrm{i} n, \quad \forall n \neq 0,
$$

whereas the brackets between all other coordinate functions vanish, and which has the property that for any $s \in \mathbb{N}$, the restriction of $\Phi^{k d v}$ to $H_{0}^{s}\left(\mathbb{T}_{1}\right)$ is a real analytic diffeomorphism with range $h_{0}^{s}, \Phi^{k d v}: H_{0}^{s}\left(\mathbb{T}_{1}\right) \rightarrow h_{0}^{s}$, so that the KdV Hamiltonian, when expressed in the coordinates $w_{n}, n \neq 0$, is in normal form. More precisely,

$$
H^{k d v} \circ \Psi^{k d v}: h_{0}^{1} \rightarrow \mathbb{R}, \quad \Psi^{k d v}:=\left(\Phi^{k d v}\right)^{-1},
$$

is a real analytic function $\mathcal{H}^{k d v}$ of the actions $I(w)=\left(I_{n}(w)\right)_{n \geq 1}$ alone,

$$
\mathcal{H}^{k d v}: \ell_{+}^{1,3} \rightarrow \mathbb{R}, I \mapsto \mathcal{H}^{k d v}(I), \quad I_{n}(w):=2 \pi n w_{n} w_{-n}, \forall n \geq 1,
$$

where $\ell_{+}^{1,3}$ denotes the positive quadrant of the weighted $\ell^{1}-$ sequence space,

$$
\ell^{1,3} \equiv \ell^{1,3}(\mathbb{N}, \mathbb{R}):=\left\{I=\left(I_{n}\right)_{n \geq 1} \subset \mathbb{R}: \sum_{n=1}^{\infty} n^{3}\left|I_{n}\right|<\infty\right\}, \quad \mathbb{N}:=\mathbb{Z}_{\geq 1} .
$$


Equation (1.2), when expressed in the coordinates $w_{n}, n \neq 0$, then takes the form

$$
\dot{w}_{n}=\mathrm{i} \omega_{n}^{k d v}(I) w_{n}, \quad \forall n \neq 0,
$$

where $\omega_{n}^{k d v}(I), n \neq 0$, denote the $\mathrm{KdV}$ frequencies

$$
\omega_{n}^{k d v}(I):=\partial_{I_{n}} \mathcal{H}^{k d v}(I), \quad \omega_{-n}^{k d v}(I):=-\omega_{n}^{k d v}(I), \quad \forall n \geq 1 .
$$

Since by (1.10) the action variables Poisson commute, $\left\{I_{n}, I_{m}\right\}, \forall n, m \geq 1$, it follows that they are prime integrals of (1.2) and so are the frequencies $\omega_{n}^{k d v}(\bar{I}), n \neq 0$. As a consequence, (1.11) can be solved by quadrature. Finally, the differential $d_{0} \Phi^{k d v}$ : $L_{0}^{2}\left(\mathbb{T}_{1}\right) \rightarrow \ell_{0}^{2}$ of $\Phi^{k d v}$ at $q=0$ is the Fourier transform (cf. [28], Theorem 9.8)

$$
\mathcal{F}: L_{0}^{2}\left(\mathbb{T}_{1}\right) \rightarrow \ell_{0}^{2}, \quad q \mapsto\left(q_{n}\right)_{n \neq 0}, \quad q_{n}:=\int_{0}^{1} q(x) e^{-2 \pi \mathrm{i} n x} d x,
$$

and hence $d_{0} \Psi^{k d v}$ is given by the inverse Fourier transform $\mathcal{F}^{-1}$. We remark that the coordinates $w_{ \pm n} \equiv w_{ \pm n}(q)$, referred to as (complex) Birkhoff coordinates, are related to the (real) Birkhoff coordinates $x_{n}, y_{n}, n \geq 1$, introduced in [28], by

$$
x_{n}=\frac{w_{n}+w_{-n}}{2 \sqrt{n \pi}}, \quad y_{n}=\mathrm{i} \frac{w_{n}-w_{-n}}{2 \sqrt{n \pi}}, \quad \forall n \geq 1,
$$

where $\sqrt{\cdot}$ denotes the principal branch of the square root, $\sqrt{\cdot} \equiv \sqrt[+]{\cdot}$.

The Birkhoff coordinates are well suited to describe the finite gap solutions of (1.2). For any finite subset $S_{+} \subseteq \mathbb{N}$, let

$$
S:=S_{+} \cup\left(-S_{+}\right), \quad S^{\perp}:=\mathbb{Z} \backslash(S \cup\{0\}) .
$$

We denote by $M_{S}$ the submanifold of $L_{0}^{2}\left(\mathbb{T}_{1}\right)$, given by

$$
M_{S}:=\left\{q=\Psi^{k d v}(w): w_{n}(q)=0 \forall n \in S^{\perp}\right\},
$$

whose elements are referred to as $S$-gap potentials, and by $M_{S}^{o}$ the open subset of $M_{S}$, consisting of the so called proper $S$-gap potentials,

$$
M_{S}^{o}:=\left\{q \in M_{S}: w_{n}(q) \neq 0 \forall n \in S\right\} .
$$

Note that $M_{S}$ is contained in $\cap_{s \geq 0} H_{0}^{s}\left(\mathbb{T}_{1}\right)$ and hence consists of $C^{\infty}$-smooth potentials and that $M_{S}^{o}$ can be parametrized by the action-angle coordinates $\theta=\left(\theta_{k}\right)_{k \in S_{+}} \in \mathbb{T}^{S_{+}}$, and $I=\left(I_{k}\right)_{k \in S_{+}} \in \mathbb{R}_{>0}^{S_{+}}$,

$$
\Psi_{S_{+}}: \mathcal{M}_{S}^{o}:=\mathbb{T}^{S_{+}} \times \mathbb{R}_{>0}^{S_{+}} \rightarrow M_{S}^{o},(\theta, I) \mapsto \Psi_{S_{+}}(\theta, I):=\Psi^{k d v}(w(\theta, I))
$$

where $\mathbb{T}:=\mathbb{R} / 2 \pi \mathbb{Z}$ and $w(\theta, I)=\left(w_{n}(\theta, I)\right)_{n \neq 0}$ is defined by

$$
w_{ \pm n}:=\sqrt{I_{n} /(2 \pi n)} e^{\mp \mathrm{i} \theta_{n}}, \quad \forall n \in S_{+}, \quad w_{n}:=0, \quad \forall n \in S^{\perp} .
$$

Introduce

$$
h_{\perp}^{s}:=\left\{w \in h_{\perp c}^{s}: w_{-n}=\bar{w}_{n} \forall n \in S^{\perp}\right\}, \quad h_{\perp c}^{s}:=h^{s}\left(S^{\perp}, \mathbb{C}\right) .
$$


For notational convenience, we view $\mathcal{M}_{S}^{o} \times h_{\perp}^{s}$ as a subset of $h_{0}^{s}$. Its elements are denoted by

$$
\theta=\left(\theta_{n}\right)_{n \in S_{+}}, \quad I=\left(I_{n}\right)_{n \in S_{+}}, \quad w=\left(w_{n}\right)_{n \in S^{\perp}}
$$

and it is endowed with the canonical Poisson bracket, given by

$$
\left\{I_{n}, \theta_{n}\right\}=1, \quad \forall n \in S_{+}, \quad\left\{w_{n}, w_{-n}\right\}=\mathrm{i} 2 \pi n, \quad \forall n \in S_{+}^{\perp}:=S^{\perp} \cap \mathbb{N},
$$

whereas the brackets between all other coordinate functions vanish.

It is convenient to introduce the frequency vector $\omega(I)$ (cf. (1.12)),

$$
\omega(I):=\left(\omega_{n}^{k d v}(I, 0)\right)_{n \in S_{+}} .
$$

By [11], the action to frequency map $\omega: \mathbb{R}_{>0}^{S_{+}} \rightarrow \mathbb{R}^{S_{+}}, I \mapsto \omega(I)$, is a local diffeomorphism. Throughout the paper, we denote by $\Xi \subset \mathbb{R}_{>0}^{S_{+}}$the closure of a bounded, open, nonempty set so that the restriction of $\omega$ to $\Xi$ is a diffeomorphism onto its image $\Pi:=\omega(\Xi)$ and so that for some $\delta>0$,

$$
\Xi+B_{S_{+}}(\delta) \subset \mathbb{R}_{>0}^{S_{+}},
$$

where $B_{S_{+}}(\delta)$ is the ball in $\mathbb{R}^{S_{+}}$of radius $\delta>0$, centered at the origin. We remark that for any $I \in \Xi+B_{S_{+}}(\delta)$, the nth action $I_{n}=I_{n}(w), n \in S_{+}$, is of the form $I_{n}(w)=I_{n}^{(0)}+y$ where $I_{n}^{(0)}:=2 \pi n w_{n}^{(0)} w_{-n}^{(0)} \in \Xi$ and

$$
y_{n}=\left(w_{n}-w_{n}^{(0)}\right) w_{-n}^{(0)}+w_{n}^{(0)}\left(w_{-n}-w_{-n}^{(0)}\right)+\left(w_{n}-w_{n}^{(0)}\right)\left(w_{-n}-w_{-n}^{(0)}\right) .
$$

The inverse of $\omega: \Xi \rightarrow \Pi$ is denoted by $\mu$,

$$
\mu: \Pi \rightarrow \Xi, \quad \omega \mapsto \mu(\omega) .
$$

In what follows, we will consider the frequency vector $\omega$ as a parameter. For any $\omega \in \Pi$, a $S$-gap solution of (1.2) is defined as a solution of the form

$$
q(t, x ; \omega)=\Psi_{S_{+}}\left(\theta^{(0)}+\omega t, \mu(\omega)\right)(x), \quad \theta^{(0)} \in \mathbb{T}^{S_{+}},
$$

whereas a finite gap solution of (1.2) is a solution of the form (1.16) for some $S=$ $S_{+} \cup\left(-S_{+}\right)$with $S_{+} \subset \mathbb{N}$ finite. The $S$-gap solution $t \mapsto q(t, x ; \omega)$ is a curve on the $\left|S_{+}\right|$-dimensional torus

$$
\mathfrak{T}_{\mu(\omega)}:=\Psi_{S_{+}}\left(\mathbb{T}^{S_{+}} \times\{\mu(\omega)\}\right)
$$

We note that $\mathfrak{T}_{\mu(\omega)}$ is invariant under (1.2) and Lyapunov stable in $H_{0}^{s}\left(\mathbb{T}_{1}\right)$ for any $s \geq 0$. More precisely, for any $\varepsilon>0$ there exists $\delta>0$, depending on $s$, so that for any initial data $u_{0} \in H_{0}^{s}\left(\mathbb{T}_{1}\right)$ with

$$
\operatorname{dist}_{H^{s}}\left(u_{0}, \mathfrak{T}_{\mu(\omega)}\right) \leq \delta, \quad \operatorname{dist}_{H^{s}}\left(u_{0}, \mathfrak{T}_{\mu(\omega)}\right):=\inf _{q \in \mathfrak{T}_{\mu(\omega)}}\left\|u_{0}-q\right\|_{s},
$$

the solution $u(t, \cdot)$ of $(1.2)$ with $u(0, \cdot)=u_{0}$ satisfies

$$
\operatorname{dist}_{H^{s}}\left(u(t, \cdot), \mathfrak{T}_{\mu(\omega)}\right) \leq \varepsilon, \quad \forall t \in \mathbb{R} .
$$


Finally, we introduce the so called normal frequencies,

$$
\Omega_{j}(\omega):=\omega_{j}^{k d v}(\mu(\omega), 0), \quad j \in S^{\perp}, \omega \in \Pi,
$$

and for any given $\tau>\left|S_{+}\right|$, the subsets $\Pi_{\gamma}$ of $\Pi$,

$$
\Pi_{\gamma}:=\cap_{i=0}^{3} \Pi_{\gamma}^{(i)}, \quad 0<\gamma<1,
$$

where $\Pi_{\gamma}^{(i)}, 0 \leq i \leq 3$, are given by

$$
\begin{aligned}
\Pi_{\gamma}^{(0)} & :=\left\{\omega \in \Pi:|\omega \cdot \ell| \geq \frac{\gamma}{\langle\ell\rangle^{\tau}} \forall \ell \in \mathbb{Z}^{S_{+}} \backslash\{0\}\right\} \\
\Pi_{\gamma}^{(1)} & :=\left\{\omega \in \Pi:\left|\omega \cdot \ell+\Omega_{j}(\omega)\right| \geq \frac{\gamma}{\langle\ell\rangle^{\tau}} \forall(\ell, j) \in \mathbb{Z}^{S_{+}} \times S^{\perp}\right\} \\
\Pi_{\gamma}^{(2)} & :=\left\{\omega \in \Pi:\left|\omega \cdot \ell+\Omega_{j_{1}}(\omega)+\Omega_{j_{2}}(\omega)\right| \geq \frac{\gamma}{\langle\ell\rangle^{\tau}}\right. \\
& \left.\forall\left(\ell, j_{1}, j_{2}\right) \in \mathbb{Z}^{S_{+}} \times S^{\perp} \times S^{\perp} \text { with }\left(\ell, j_{1}, j_{2}\right) \neq\left(0, j_{1},-j_{1}\right)\right\}, \\
\Pi_{\gamma}^{(3)} & :=\left\{\omega \in \Pi:\left|\omega \cdot \ell+\Omega_{j_{1}}(\omega)+\Omega_{j_{2}}(\omega)+\Omega_{j_{3}}(\omega)\right| \geq \frac{\gamma}{\langle\ell\rangle^{\tau}\left\langle j_{1}\right\rangle^{2}\left\langle j_{2}\right\rangle^{2}\left\langle j_{3}\right\rangle^{2}}\right. \\
& \left.\forall\left(\ell, j_{1}, j_{2}, j_{3}\right) \in \mathbb{Z}^{S_{+}} \times S^{\perp} \times S^{\perp} \times S^{\perp} \text { with } j_{k}+j_{m} \neq 0 \forall k, m \in\{1,2,3\}\right\} .
\end{aligned}
$$

Here we used the standard notation for vectors $y$ in $\mathbb{R}^{n}$,

$$
\langle y\rangle:=\max \{1,|y|\}, \quad|y|:=\left(\sum_{j=1}^{n}\left|y_{j}\right|^{2}\right)^{1 / 2}, \quad \forall y \in \mathbb{R}^{n} .
$$

We refer to $\Pi_{\gamma}^{(j)}, 0 \leq j \leq 3$, as the $j$ th Melnikov conditions and note that the third Melnikov conditions allow for 'a loss of derivatives in space'- - see item ( $i i$ ) in Comments on Theorem 1.1 below.

The goal of this paper is to prove a long time stability result of finite gap solutions (1.16) of the Korteweg-de Vries equation on $\mathbb{T}_{1}$. To state it, we denote for any Banach space $X$ with norm $\|\cdot\|_{X}$, integer $m \geq 0$, and interval $J \subset \mathbb{R}$, by $C^{m}(J, X)$ the Banach space of functions $f: J \rightarrow X$, which are $m$ times continuously differentiable, endowed with the supremum norm, $\|f\|_{C_{t}^{m}}:=\max _{0 \leq j \leq m} \sup \left\{\left\|\partial_{t}^{j} f(t)\right\|_{X}: t \in J ; 0 \leq j \leq m\right\}$.

Theorem 1.1. Let $f$ be a function in $C^{\infty}\left(\mathbb{T}_{1} \times \mathbb{R}\right)\left(c f\right.$. (1.6)), $S_{+}$be a finite subset of $\mathbb{N}$, and $\tau$ be a number with $\tau>\left|S_{+}\right|(c f$. (1.20)). Then for any integer s sufficiently large and any $\omega \in \Pi_{\gamma}, 0<\gamma<1$, there exists $0<\varepsilon_{0} \equiv \varepsilon_{0}(s, \gamma)<1$ with the following properties: for any $0<\varepsilon \leq \varepsilon_{0}$ and any initial data $u_{0} \in H_{0}^{S}\left(\mathbb{T}_{1}\right)$, satisfying

$$
\operatorname{dist}_{H^{s}}\left(u_{0}, \mathfrak{T}_{\mu(\omega)}\right) \leq \varepsilon,
$$

equation (1.4) admits a unique solution

$$
t \mapsto u(t, \cdot) \operatorname{in} C^{0}\left([-T, T], H_{0}^{s}\left(\mathbb{T}_{1}\right)\right) \cap C^{1}\left([-T, T], H_{0}^{s-3}\left(\mathbb{T}_{1}\right)\right)
$$

with initial data $u(0, x)=u_{0}(x)$ and $T \equiv T_{\varepsilon, s, \gamma}=O\left(\varepsilon^{-2}\right)$. Moreover, $u$ satisfies the estimate

$$
\operatorname{dist}_{H^{s}}\left(u(t, \cdot), \mathfrak{T}_{\mu(\omega)}\right) \lesssim_{s, \gamma} \varepsilon, \quad \forall-T \leq t \leq T
$$


where the distance function dist $_{H^{s}}$ is defined in (1.17). Furthermore, there exists $0<$ a $<1$ so that for any $0<\gamma<1$, the Lebesgue measure $\left|\Pi \backslash \Pi_{\gamma}\right|$ of $\Pi \backslash \Pi_{\gamma}$ satisfies

$$
\left|\Pi \backslash \Pi_{\gamma}\right| \lesssim \gamma^{\mathrm{a}}, \quad \text { implying that } \lim _{\gamma \rightarrow 0}\left|\Pi_{\gamma}\right|=|\Pi| .
$$

Here and in the sequel, the notation $h \lesssim \alpha, \ldots g$ means that the real valued function $h$, depending on various variables, satisfies an estimate of the form $h \leq C g$ where $g$ is also a real valued function, typically small, and the constant $C>0$ only depends on the parameters $\alpha, \ldots$. For notational convenience, the dependence of the constant $C$ on $f$, $S_{+}$, and $\tau$ is not indicated.

\section{Comments on Theorem 1.1}

(i) Initial data. Note that the size of the distance of the initial value $u_{0}$ to the considered $S$-gap solution of the KdV equation (cf. (1.22)) is assumed to be of the same order of magnitude as the size of the perturbation $\varepsilon F(u)$ in (1.4).

(ii) Measure estimate (1.23). The proof of the measure estimates (1.23) requires that the third Melnikov conditions $\Pi_{\gamma}^{(3)}$ in (1.20) allow for a loss of derivatives in space. Furthermore, a key ingredient into the proof of (1.23) is the case $n=3$ of Fermat's Last Theorem, proved by Euler [21] (cf. Lemma 8.3).

(iii) Assumptions in Theorem 1.1. The results of Theorem 1.1 hold for any density $f(x, \zeta)$ of class $\mathcal{C}^{\sigma}$ with $\sigma$ sufficiently large. Furthermore, corresponding results hold for (invariant tori of) finite gap solutions of the $\mathrm{KdV}$ equation in the affine spaces $c+H_{0}^{s}\left(\mathbb{T}_{1}\right), c \in \mathbb{R}$. We assume in this paper that $f$ is $\mathcal{C}^{\infty}-$ smooth and that $c=0$ merely to simplify the exposition.

In order to limit the size of the paper, we assume the perturbation $\varepsilon F(u)$ to be semilinear (cf. (1.5)), leaving the case of a quasilinear one for future work. Most likely, the elaborate method designed in [23] will allow to transform quasilinear perturbations into normal form while preserving the Hamiltonian structure of the equation.

(iv) Time of stability. It seems unlikely that the stability results of Theorem 1.1 in the generality stated are valid for time intervals of size larger than $O\left(\varepsilon^{-2}\right)$ since the conditions, required to hold for the frequencies $\Omega_{j}, j \in S^{\perp}$, so that the normal form procedure could be implemented, are too strong. See Remark 8.1 at the end of Sect. 8. Actually, it might be possible that the (almost) resonances of the KdV frequencies of degree four can be used to prove instability results for solutions of the perturbed equation (1.4)—see [16,25] and references therein for related results for Schrödinger equations in two space dimension.

(v) Conservation of momentum. If the density $f$ of the perturbation $P_{f}(u)=\int_{0}^{1}$ $f(x, u(x)) d x$ does not explicitely depend on $x$, then the momentum $M(u):=$ $\frac{1}{2} \int_{\mathbb{T}_{1}} u^{2} d x$ is a prime integral of Eq. (1.4). We plan to prove in future work that the stability time can be improved in such a case.

(v) Integrable PDEs. The method of proof of Theorem 1.1 is quite general. We expect that for any integrable PDE, admitting coordinates of the type constructed in [27], a corresponding version of Theorem 1.1 holds, up to the measure estimates related to the nonresonance conditions for the frequencies of the integrable PDE considered. These estimates might require specific arithmetic properties of the frequenciessee item (ii) above.

To explain the main ideas of the proof, we first need to introduce some terminology and additional notations. They will be used throughout the paper. 
Notations and terminology. For any finite subset $S_{+} \subset \mathbb{N}, L_{\perp}^{2}\left(\mathbb{T}_{1}\right)$ is the subspace, given by

$$
L_{\perp}^{2}\left(\mathbb{T}_{1}\right):=\left\{w=\sum_{n \in S^{\perp}} w_{n} e^{\mathrm{i} 2 \pi n x} \in L_{0}^{2}\left(\mathbb{T}_{1}\right)\right\}, \quad S^{\perp}=\mathbb{Z} \backslash\left(S_{+} \cup\left(-S_{+}\right) \cup\{0\}\right),
$$

and $\Pi_{\perp}$ denotes the $L^{2}-$ orthogonal projector onto the subspace $L_{\perp}^{2}\left(\mathbb{T}_{1}\right)$. For any $s>0$, we set

$$
H_{\perp}^{s}\left(\mathbb{T}_{1}\right):=H^{s}\left(\mathbb{T}_{1}\right) \cap L_{\perp}^{2}\left(\mathbb{T}_{1}\right), \quad H_{\perp}^{0}\left(\mathbb{T}_{1}\right):=L_{\perp}^{2}\left(\mathbb{T}_{1}\right) .
$$

By $\mathcal{E}_{s}$ we denote the phase space and by $E_{S}$ the corresponding tangent space, given by $\mathcal{E}_{s}:=\mathbb{T}^{S_{+}} \times \mathbb{R}^{S_{+}} \times H_{\perp}^{s}\left(\mathbb{T}_{1}\right), \quad \mathcal{E} \equiv \mathcal{E}_{0}, \quad E_{s}:=\mathbb{R}^{S_{+}} \times \mathbb{R}^{S_{+}} \times H_{\perp}^{s}\left(\mathbb{T}_{1}\right), \quad E \equiv E_{0}$,

where $\mathbb{T}_{1}=\mathbb{R} / \mathbb{Z}$ and $\mathbb{T}=\mathbb{R} / 2 \pi \mathbb{Z}$. Elements of $\mathcal{E}$ are denoted by $\mathfrak{x}=(\theta, y, w)$ and the ones of its tangent space $E$ by $\widehat{x}=(\widehat{\theta}, \widehat{y}, \widehat{w})$. For $s>0, H_{\perp}^{s}\left(\mathbb{T}_{1}\right)^{*}$ denotes the dual space of $H_{\perp}^{s}\left(\mathbb{T}_{1}\right)$, which is canonically identified with the Sobolev space $H_{\perp}^{-s}\left(\mathbb{T}_{1}\right)$ of distributions. The spaces $\mathcal{E}_{-s}$ and $E_{-s}$ are then defined as in (1.26). On $E$, we denote by $\langle\cdot, \cdot\rangle_{E}$ the inner product defined by

$$
\left\langle\left(\widehat{\theta}_{1}, \widehat{y}_{1}, \widehat{w}_{1}\right),\left(\widehat{\theta}_{2}, \widehat{y}_{2}, \widehat{w}_{2}\right)\right\rangle_{E}:=\widehat{\theta}_{1} \cdot \widehat{\theta}_{2}+\widehat{y}_{1} \cdot \widehat{y}_{2}+\left\langle\widehat{w}_{1}, \widehat{w}_{2}\right\rangle
$$

where $\langle\cdot, \cdot\rangle$ is the standard real scalar product on $L_{\perp}^{2}$. For notational convenience, $\Pi_{\perp}$ also denotes the projector of $E_{s}$ onto its third component,

$$
\Pi_{\perp}: E_{s} \rightarrow H_{\perp}^{s}\left(\mathbb{T}_{1}\right), \quad(\widehat{\theta}, \widehat{y}, \widehat{w}) \mapsto \widehat{w} .
$$

For any $0<\delta<1$, we denote by $B_{S_{+}}(\delta)$ the open ball in $\mathbb{R}^{S_{+}}$of radius $\delta$ centered at 0 and by $B_{\perp}^{s}(\delta), s \geq 0$, the corresponding one in $H_{\perp}^{s}\left(\mathbb{T}_{1}\right)$. For $s=0$, we also write $B_{\perp}(\delta)$ instead of $B_{\perp}^{0}(\delta)$. These balls are used to define the following open neighborhoods in $\mathcal{E}_{s}, s \geq 0$,

$$
\mathcal{V}^{S}(\delta):=\mathbb{T}_{1}^{S_{+}} \times B_{S_{+}}(\delta) \times B_{\perp}^{S}(\delta), \quad \mathcal{V}(\delta) \equiv \mathcal{V}^{0}(\delta), \quad 0<\delta<1 .
$$

For notational convenience, often without stating it explicitly, $\delta>0$ will take on different values in the course of our arguments. In particular, $\delta>0$ typically will depend on $s$. (Note that by (1.15), the coordinates $y=\left(y_{n}\right)_{n \in S_{+}}$are of the same order as the coordinates $w=\left(w_{n}\right)_{\left.n \in S^{\perp} .\right)}$

For any $k \geq 1, \partial_{x}^{-k}: L^{2}\left(\mathbb{T}_{1}\right) \rightarrow L_{0}^{2}\left(\mathbb{T}_{1}\right)$ is the linear operator, defined by

$$
\partial_{x}^{-k}\left[e^{2 \pi \mathrm{i} n x}\right]=\frac{1}{(2 \pi \mathrm{i} n)^{k}} e^{2 \pi \mathrm{i} n x}, \quad \forall n \neq 0, \quad \text { and } \quad \partial_{x}^{-k}[1]=0 .
$$

The space $\mathcal{V}^{S}(\delta)$ is endowed with the symplectic form

$$
\mathcal{W}:=\left(\sum_{j \in S_{+}} d y_{j} \wedge d \theta_{j}\right) \oplus \mathcal{W}_{\perp}
$$

where $\mathcal{W}_{\perp}$ is the restriction to $L_{\perp}^{2}\left(\mathbb{T}_{1}\right)$ of the symplectic form $\mathcal{W}_{L_{0}^{2}}$ defined in (1.9). Throughout the paper, the Hamiltonians considered depend on the small parameter $\varepsilon \in$ 
$\left[0, \varepsilon_{0}\right], 0<\varepsilon_{0}<1$, and are $C^{\infty}$-smooth maps, $\mathcal{V}^{s}(\delta) \times\left[0, \varepsilon_{0}\right] \rightarrow \mathbb{R}$. Given such a Hamiltonian $H$, we often do not indicate the dependence of $H$ on the parameter $\varepsilon$. The Hamiltonian vector field of $H$ is denoted by $X_{H}$. It is given by

$$
X_{H}(\mathfrak{x})=\mathcal{J} \nabla H(\mathfrak{x})=\left(-\nabla_{y} H(\mathfrak{x}), \nabla_{\theta} H(\mathfrak{x}), \partial_{x} \nabla_{\perp} H(\mathfrak{x})\right)
$$

where $\mathcal{J}$ is the Poisson structure, associated to the symplectic form $\mathcal{W}$,

$$
\mathcal{J}: E_{S} \rightarrow E_{s-1}, \quad(\widehat{\theta}, \widehat{y}, \widehat{w}) \mapsto\left(-\widehat{y}, \widehat{\theta}, \partial_{x} \widehat{w}\right)
$$

and where $\nabla_{\perp} H(\mathfrak{x}) \equiv \nabla_{w} H(\mathfrak{x})$ denotes the $L^{2}$-gradient of $H$ with respect to the variable $w$. For notational convenience, we denote by $\{F, G\}$ the Poisson bracket corresponding to $\mathcal{J}$,

$$
\begin{aligned}
\{F, G\} & =\mathcal{W}\left(X_{F}, X_{G}\right)=\langle\nabla F, \mathcal{J} \nabla G\rangle_{E} \\
& =-\nabla_{\theta} F \cdot \nabla_{y} G+\nabla_{y} F \cdot \nabla_{\theta} G+\left\langle\nabla_{\perp} F, \partial_{x} \nabla_{\perp} G\right\rangle .
\end{aligned}
$$

Given a Hamiltonian vector field $X_{F}: \mathcal{V}^{s}(\delta) \times\left[0, \varepsilon_{0}\right] \rightarrow E_{s}$ with Hamiltonian $F$, we denote by $\Phi_{F}(\tau, \cdot)$ or $\Phi_{X_{F}}(\tau, \cdot)$ the flow generated by $X_{F}$. For the vector fields $X_{F}$ considered in this paper, there exists $0<\delta^{\prime}<\delta$ so that for any $\tau \in[-1,1]$, the flow map $\mathcal{V}^{S}\left(\delta^{\prime}\right) \rightarrow \mathcal{V}^{S}(\delta), \mathfrak{x} \mapsto \Phi_{F}(\tau, \mathfrak{x})$ is well defined. The Taylor expansion of $\tau \mapsto H \circ \Phi_{F}(\tau, \mathfrak{x})$ at $\tau=0$ can be computed as

$$
H \circ \Phi_{F}(\tau, \mathfrak{x})=H(\mathfrak{x})+\tau\{H, F\}(\mathfrak{x})+\tau^{2} \int_{0}^{1}(1-t)\{\{H, F\}, F\} \circ \Phi_{F}(t \tau, \mathfrak{x}) d t
$$

We will also need to consider $C^{\infty}$-smooth vector fields, which are not necessarily Hamiltonian,

$$
X=\left(X^{(\theta)}, X^{(y)}, X^{\perp}\right): \mathcal{V}^{s}(\delta) \times\left[0, \varepsilon_{0}\right] \rightarrow E_{S},
$$

where $X^{(\theta)}, X^{(y)}$, and $X^{\perp}$ are the components of $X$,

$$
X^{(\theta)}, X^{(y)}: \mathcal{V}^{s}(\delta) \times\left[0, \varepsilon_{0}\right] \rightarrow \mathbb{R}^{S_{+}}, \quad X^{\perp}: \mathcal{V}^{s}(\delta) \times\left[0, \varepsilon_{0}\right] \rightarrow H_{\perp}^{s}\left(\mathbb{T}_{1}\right) .
$$

The corresponding flow is denoted by $\Phi_{X}(\tau, \cdot)$. Again we will only consider vector fields $X$ with the property that there exists $0<\delta^{\prime}<\delta$ so that for any $\tau \in[-1,1], \Phi_{X}(\tau, \cdot)$ is well defined on $\mathcal{V}^{s}\left(\delta^{\prime}\right)$. Given two $C^{\infty}$-smooth vector fields $X, Y: \mathcal{V}^{s}(\delta) \times\left[0, \varepsilon_{0}\right] \rightarrow E_{s}$, the commutator $[X, Y]$ is defined as

$$
[X, Y](\mathfrak{x}):=d X(\mathfrak{x})[Y(\mathfrak{x})]-d Y(\mathfrak{x})[X(\mathfrak{x})] .
$$

The pull-back of a vector field $X: \mathcal{V}^{s}(\delta) \rightarrow E_{s}$ by a $C^{\infty}$-smooth diffeomorphism $\Phi: \mathcal{V}^{s}\left(\delta^{\prime}\right) \rightarrow \mathcal{V}^{s}(\delta)$ is defined as,

$$
\Phi^{*} X(\mathfrak{x}):=d \Phi(\mathfrak{x})^{-1} X(\Phi(\mathfrak{x})), \quad \forall \mathfrak{x} \in \mathcal{V}^{s}\left(\delta^{\prime}\right) .
$$

If $\Phi_{\tau}(\cdot) \equiv \Phi_{Y}(\tau, \cdot)$ is the flow of a vector field $Y$, then the Taylor expansion of $\tau \mapsto$ $\Phi_{\tau}^{*} X(\mathfrak{x})$ at $\tau=0$ reads

$$
\Phi_{\tau}^{*} X(\mathfrak{x})=X(\mathfrak{x})+\tau \int_{0}^{1}(d \Phi(t \tau, \mathfrak{x}))^{-1}[X, Y](\Phi(t \tau, \mathfrak{x})) d t
$$




$$
=X(\mathfrak{x})+\tau[X, Y](\mathfrak{x})+\tau^{2} \int_{0}^{1}(1-t)(d \Phi(t \tau, \mathfrak{x}))^{-1}[[X, Y], Y](\Phi(t \tau, \mathfrak{x})) d t
$$

In the case $\tau=1$, we will often write $\Phi_{Y}^{*} X$ instead of $\Phi_{1}^{*} X$. Clearly if $X=X_{H}, Y=Y_{F}$ are Hamiltonian vector fields, then

$$
[X, Y]=X_{\{H, F\}}, \quad\left(\Phi_{Y}(\tau, \cdot)\right)^{*} X=X_{H \circ \Phi_{Y}(\tau, \cdot)} .
$$

Given two linear operators $A, B$, acting on $L^{2}\left(\mathbb{T}_{1}\right)$ (or $L_{\perp}^{2}\left(\mathbb{T}_{1}\right)$ ), their commutator is conveniently denoted by $[A, B]_{l i n}$,

$$
[A, B]_{l i n}=A B-B A .
$$

Moreover, given a densely defined linear operator $A: L_{\perp}^{2}\left(T_{1}\right) \rightarrow L_{\perp}^{2}\left(\mathbb{T}_{1}\right)$, whose domain contains the elements of the Fourier basis $e^{\mathrm{i} 2 \pi j x}, j \in S^{\perp}$, we denote by $A_{j}^{j^{\prime}}$ or $[A]_{j}^{j^{\prime}}$ the (Fourier) matrix coefficients of $A$,

$$
A_{j}^{j^{\prime}}:=\int_{0}^{1} A\left[e^{\mathrm{i} 2 \pi j^{\prime} x}\right] e^{-\mathrm{i} 2 \pi j x} d x, \quad j, j^{\prime} \in S^{\perp} .
$$

Given a Banach space $\left(X,\|\cdot\|_{X}\right)$, we denote by $C_{b}^{\infty}\left(\mathcal{V}^{s}(\delta) \times\left[0, \varepsilon_{0}\right], X\right)$ the space of $C^{\infty}$ functions $\mathcal{V}^{s}(\delta) \times\left[0, \varepsilon_{0}\right] \rightarrow X$ with all derivatives bounded.

In our normal form procedure, we need to take into account the order of vanishing with respect to the variables $y, w$ and the small parameter $\varepsilon$. The following definition turns out to be convenient.

Definition 1.1. Let $\left(B,\|\cdot\|_{B}\right)$ be a Banach space and $p \in \mathbb{Z}_{\geq 0}$. A $C^{\infty}$-smooth map

$$
g: \mathcal{V}^{s}(\delta) \times\left[0, \varepsilon_{0}\right] \rightarrow B,(\mathfrak{x}, \varepsilon) \mapsto g(\mathfrak{x}, \varepsilon)
$$

is said to be small of order $p$ if for any $\beta \in \mathbb{Z}_{\geq 0}^{S_{+}}$and $k_{1}, k_{2} \in \mathbb{Z}_{\geq 0}$ with $|\beta|+k_{1}+k_{2} \leq p-1$

$$
d_{\perp}^{k_{2}} \partial_{y}^{\beta} \partial_{\varepsilon}^{k_{1}} g(\theta, 0,0,0)=0, \quad \forall \theta \in \mathbb{T}^{S_{+}} .
$$

Note that if $g$ is small of order $p$, then

$$
\|g(\mathfrak{x}, \varepsilon)\|_{B} \lesssim_{g}\left(|y|+\|w\|_{s}+\varepsilon\right)^{p}, \quad \forall \mathfrak{x}=(\theta, y, w) \in \mathcal{V}^{S}(\delta), \forall \varepsilon \in\left[0, \varepsilon_{0}\right],
$$

and for any $\alpha \in \mathbb{Z}_{>0}^{S_{+}}$, $\partial_{\theta}^{\alpha} g$ is small of order $p$ as well.

Given two Banach spaces $\left(X,\|\cdot\|_{X}\right),\left(Y,\|\cdot\|_{Y}\right)$, we denote by $\mathcal{B}(X, Y)$ the space of bounded linear operators $X \rightarrow Y$. If $X=Y$, we write $\mathcal{B}(X)$ instead of $\mathcal{B}(X, X)$. Moreover for any integer $p \geq 2$, we denote by $\mathcal{B}_{p}(X, Y)$, the space of bounded, $p$ multilinear maps $M: X^{p} \rightarrow Y$, equipped with the standard norm,

$$
\|M\|_{\mathcal{B}_{p}(X, Y)}:=\sup _{\left\|u_{1}\right\|_{X}, \ldots,\left\|u_{p}\right\|_{X} \leq 1}\left\|M\left[u_{1}, \ldots, u_{p}\right]\right\|_{Y}, \quad M \in \mathcal{B}_{p}(X, Y) .
$$

If $X=Y$, we write $\mathcal{B}_{p}(X)$ instead of $\mathcal{B}_{p}(X, X)$. Furthermore, given open sets $U \subset X$ and $V \subset Y$, we denote by $C_{b}^{\infty}(U, V)$ the space of maps $f: U \rightarrow V$ which are $C^{\infty}$-smooth and together with each of its derivatives, bounded.

Overview of the proof of Theorem 1.1. We prove Theorem 1.1 by the means of a normal form procedure. A key ingredient are canonical coordinates near a torus $\mathfrak{T}_{\mu(\omega)}$ 
of arbitrary size, constructed in [27]. They are obtained by first linearizing the Birkhoff map $\Phi^{k d v}$ at $\mathfrak{T}_{\mu(\omega)}$ and then constructing a symplectic corrector. The new coordinates yield a family of canonical transformations $\Phi_{\mu}^{k d v}$, parametrized by $\mu \equiv \mu(\omega), \omega \in \Pi$. One of the main features of these transformations is that they admit expansions in terms of pseudo-differential operators up to a remainder of arbitrary negative order. To prove Theorem 1.1 we then follow a strategy developed in [7] in the context of water waves.

In a first step, referred to as Step 1, we write the perturbed Hamiltonian $H^{k d v}+\varepsilon P_{f}$ in the new coordinates (cf. Theorem 4.1). More precisely, in Theorem 4.1, we rephrase [27, Theorem 1.1] in a form taylored to our needs and in Corollary 4.1, we compute for any given $\mu \equiv \mu(\omega), \omega \in \Pi$, and $\mathfrak{x}=(\theta, y, w) \in \mathcal{V}^{1}(\delta)$ the Taylor expansion of $\mathcal{H}_{\varepsilon, \mu}:=\left(H^{k d v}+\varepsilon P_{f}\right) \circ \Phi_{\mu}^{k d v}$ at $(\theta, 0,0)$ up to order three in the variables $y, w$, and $\varepsilon$,

$$
\begin{aligned}
& \mathcal{H}_{\varepsilon, \mu}(\theta, y, w)=\mathcal{N}_{\mu}(y, w)+\mathcal{P}_{\varepsilon, \mu}(\theta, y, w), \\
& \mathcal{N}_{\mu}(y, w):=\omega \cdot y+\frac{1}{2} \Omega_{S_{+}}(\omega)[y] \cdot y+\frac{1}{2}\left\langle D_{\perp}^{-1} \Omega_{\perp}(\omega) w, w\right\rangle,
\end{aligned}
$$

where $\Omega_{S_{+}}(\omega)$ is given by the $S_{+} \times S_{+}$matrix $\left(\partial_{I_{j}} \omega_{i}^{k d v}(\mu, 0)\right)_{i, j \in S_{+}}$and where $D_{\perp}^{-1}$ : $L_{\perp}^{2}\left(\mathbb{T}_{1}\right) \rightarrow L_{\perp}^{2}\left(\mathbb{T}_{1}\right)$ and $\Omega_{\perp}(\omega) \equiv \Omega_{S^{\perp}}(\omega): L_{\perp}^{2}\left(\mathbb{T}_{1}\right) \rightarrow L_{\perp}^{2}\left(\mathbb{T}_{1}\right)$ are Fourier multipliers in diagonal form,

$$
D_{\perp}^{-1}[w]:=\sum_{n \in S^{\perp}} \frac{1}{2 \pi n} w_{n} e^{\mathrm{i} 2 \pi n x}, \quad \Omega_{\perp}(\omega)[w]:=\sum_{n \in S^{\perp}} \Omega_{n}(\omega) w_{n} e^{\mathrm{i} 2 \pi n x},
$$

with $\Omega_{n}(\omega)$ given by (1.18). In order to simplify notation, in the sequel, we often will not indicate the dependence of quantities such as $\mathcal{H}_{\varepsilon, \mu}, \mathcal{P}_{\varepsilon, \mu}, \Omega_{\perp}(\omega), \ldots$ on $\varepsilon, \mu \equiv \mu(\omega)$, and $\omega$.

We note that $\Omega_{\perp}$ is an unbounded operator. For any $\mathfrak{x}=(\theta, y, w), \mathcal{P}(\mathfrak{x})$ can be expanded as

$$
\mathcal{P}(\mathfrak{x})=\varepsilon\left(\mathcal{P}_{00}(\theta)+\mathcal{P}_{10}(\theta) \cdot y+\left\langle\mathcal{P}_{01}(\theta), w\right\rangle\right)+\mathcal{P}_{e}(\mathfrak{x}),
$$

where $\mathcal{P}_{e}(\mathfrak{x})$ is small of order three (cf. Definition (1.1)). The Hamiltonian vector field $X_{\mathcal{H}}$, associated to $\mathcal{H}$, is given at any point $\mathfrak{x}=(\theta, y, w)$ by

$$
X_{\mathcal{H}}(\mathfrak{x})=\left(\begin{array}{c}
-\nabla_{y} \mathcal{H}(\mathfrak{x}) \\
\nabla_{\theta} \mathcal{H}(\mathfrak{x}) \\
\partial_{x} \nabla_{\perp} \mathcal{H}(\mathfrak{x})
\end{array}\right)=\left(\begin{array}{c}
-\omega-\Omega_{S_{+}}[y]-\varepsilon \mathcal{P}_{10}(\theta)-\nabla_{y} \mathcal{P}_{e}(\mathfrak{x}) \\
\varepsilon \nabla_{\theta}\left(\mathcal{P}_{00}(\theta)+\mathcal{P}_{10}(\theta) \cdot y+\left\langle\mathcal{P}_{01}(\theta), w\right\rangle\right)+\nabla_{\theta} \mathcal{P}_{e}(\mathfrak{x}) \\
\mathrm{i} \Omega_{\perp} w+\varepsilon \partial_{x} \mathcal{P}_{01}(\theta)+\partial_{x} \nabla_{\perp} \mathcal{P}_{e}(\mathfrak{x})
\end{array}\right)
$$

We also show that the normal component $\partial_{x} \nabla_{\perp} \mathcal{P}_{e}$ of the Hamiltonian vector field $X_{\mathcal{P}_{e}}$ is the sum of a para-differential vector field of order one (cf. Definition 3.1 in Sect. 3) and a smoothing vector field (cf. Definition 3.3 in Sect. 3), i.e., for $\mathfrak{x}=(\theta, y, w)$,

$$
\partial_{x} \nabla_{\perp} \mathcal{P}_{e}(\mathfrak{x})=\Pi_{\perp} \sum_{k=0}^{N+1} T_{a_{1-k}(\mathfrak{x})} \partial_{x}^{1-k} w+\mathcal{R}_{N}^{\perp}(\mathfrak{x}),
$$

where for any $0 \leq k \leq N+1, T_{a_{1-k}(x)}$ is the operator of para-multiplication with $a_{1-k}(\mathfrak{x}) \in H^{s}\left(\mathbb{T}_{1}\right)$ (cf. (2.1) in Sect. 2), which is small of order one, and where $\mathcal{R}_{N}^{\perp}(\mathfrak{x})$ is a regularizing vector field, which is small of order two. 
In Step 2, we apply a regularization procedure, which conjugates the vector field (1.44) to another one, which is a smoothing perturbation of a vector field in diagonal form. Since the torus $\mathfrak{T}_{\mu(\omega)}$ in the coordinates $(\theta, y, w)$ is described by $\{y=0, w=0\}$, the variables $y, w$ can be used to measure the distance of a solution of the equation

$$
\left\{\begin{array}{l}
\partial_{t} \theta=-\nabla_{y} \mathcal{H} \\
\partial_{t} y=\nabla_{\theta} \mathcal{H} \\
\partial_{t} w=\partial_{x} \nabla_{\perp} \mathcal{H}
\end{array}\right.
$$

from $\mathfrak{T}_{\mu(\omega)}$. Theorem 1.1 follows from Theorem 4.2 in Sect. 4, which states that for $\mu$ in a large subset of $\Xi$ and for any initial data $\mathfrak{x}_{0}=\left(\theta_{0}, y_{0}, w_{0}\right)$, satisfying $\left|y_{0}\right|,\left\|w_{0}\right\|_{s} \leq \varepsilon$ with $s>0$ large enough, the solution $t \mapsto \mathfrak{x}(t)=(\theta(t), y(t), w(t))$ of (1.46) exists on a time interval of the form $[-T, T]$ with $T \equiv T_{\varepsilon, s, \gamma}=O\left(\varepsilon^{-2}\right)$ and

$$
|y(t)|,\|w(t)\|_{s} \lesssim s, \gamma \varepsilon, \quad \forall t \in[-T, T] .
$$

We deduce Theorem 4.2 from Theorem 4.3 and a local existence Theorem (cf. Appendix C), using energy estimates (cf. Sect. 7). Theorem 4.3 provides coordinates having the property that the vector field in (1.46), when expressed in these coordinates, is a vector field $X=\left(X^{(\theta)}, X^{(y)}, X^{\perp}\right)$ with the following two features: (F1) The $y$ component $X^{(y)}$ of $X$ is small of order three. (F2) The normal component $X^{\perp}(\mathfrak{x})$ of $X(\mathfrak{x})$ at $\mathfrak{x}=(\theta, y, w)$ reads

$$
X^{\perp}(\mathfrak{x})=\mathrm{i} \Omega_{\perp} w+\mathrm{D}^{\perp}(\mathfrak{x})[w]+\Pi_{\perp} T_{a(\mathfrak{x})} \partial_{x} w+\mathcal{R}^{\perp}(\mathfrak{x}),
$$

where $\mathrm{D}^{\perp}(\mathfrak{x})$ is a skew-adjoint Fourier multiplier of order one (depending nonlinearly on $\mathfrak{x}), a(\mathfrak{x}) \in H^{S}\left(\mathbb{T}_{1}\right)$ is small of order two, and the remainder $\mathcal{R}^{\perp}(\mathfrak{x})$ is small of order three. In broad terms, our normal form procedure diagonalizes the normal component $X^{\perp}$ of the vector field $X$ up to a term, which is small of order three and which can be controlled by energy estimates. The procedure consists in eliminating/normalizing the terms of the Taylor expansion (1.40)-(1.43) of $X_{\mathcal{H}}$, which are $p$-homogeneous in $y, w$, $\varepsilon$ with $0 \leq p \leq 2$ (cf. Definition 1.1).

Based on the normal form procedure, developed in Sects. 5 and 6, Theorem 4.3 is proved in Sect. 7. In Sect. 8 we show that the Lebesgue measure $\left|\Pi \backslash \Pi_{\gamma}\right|$ of $\Pi \backslash \Pi_{\gamma}$ (cf. (1.20)) satisfies $\left|\Pi \backslash \Pi_{\gamma}\right| \lesssim \gamma^{a}$ for some $0<a<1$. As already mentioned in item (ii) of Comments on Theorem 1.1, a key ingredient of the proof is the case $n=3$ of Fermat's Last Theorem, proved by Euler [21] (cf. Lemma 8.3). Sections 2 and 3 are prelimimary where para-differential calculus and para-differential vector fields are discussed to the extent needed in the paper.

We finish our overview of the proof of Theorem 1.1 by describing in some more detail the normal form procedure, developed in Sects. 5-6, to prove Theorem 4.3. In order to setup such a procedure in an effective way, we introduce, in the spirit of $[7,18,23]$, various classes of para-differential and smoothing vector fields, which possibly depend in a nonlinear fashion on $\mathfrak{x}=(\theta, y, w)$, and develop a symbolic calculus for them-see Sect. 3. The order of homogeneity in our symbol classes is computed with respect to $y, w, \varepsilon$ where we recall that $y, w$ (together with $\theta$ ) are phase space variables and $\varepsilon$ is the perturbation parameter appearing in (1.4) and (1.22). Our normal form procedure is split into two steps which we now describe.

In a first step, presented in Sect. 5, we normalize the terms in the Taylor expansion of the Hamiltonian $\mathcal{H}$, which are linear with respect to the normal variable $w$ and homogeneous of order at most three in $(y, w, \varepsilon)$. Equivalently, this means that we normalize the 
terms in the Taylor expansion of the Hamiltonian vector field $X_{\mathcal{H}}$ which do not contain $w$ and are homogeneous of order at most two. This is achieved by a standard normal form procedure which consists in constructing a canonical transformation, given by the time one flow map $\Phi_{\mathcal{F}}$ of a Hamiltonian vector field $X_{\mathcal{F}}$ with a Hamiltonian $\mathcal{F}$ of the form

$$
\mathcal{F}(\theta, y, w):=\mathcal{F}_{0}(\theta, y)+\left\langle\mathcal{F}_{1}(\theta, y), w\right\rangle,
$$

with the property that $X_{\mathcal{F}}$ is a smoothing Hamiltonian vector field (cf. Lemma 3.19). Hence its flow is a smoothing perturbation of the identity, implying that the Hamiltonian vector field of the Hamiltonian $\mathcal{H} \circ \Phi_{\mathcal{F}}$ has a normal component, which is again of the form (1.45) (cf. Lemma 3.17). To construct $\mathcal{F}$, we only need to impose zeroth and first Melnikov conditions on $\omega$, i.e., $\omega \in \Pi_{\gamma}^{(0)} \cap \Pi_{\gamma}^{(1)}$ (cf. (1.20)). For notational convenience, the Hamiltonian vector field obtained in this way is again denoted by $X=\left(X^{(\theta)}, X^{(y)}, X^{\perp}\right)$. The $y$-component $X^{(y)}$ is small of order three and the normal component $X^{\perp}$ of $X$ at $\mathfrak{x}=(\theta, y, w)$ has the form

$$
X^{\perp}(\mathfrak{x})=\mathrm{i} \Omega_{\perp}[w]+X_{1}^{\perp}(\theta, y)[w]+X_{2}^{\perp}(\theta)[w, w]+\text { term small of order three }
$$

where

$$
\begin{aligned}
& X_{1}^{\perp}(\theta, y)[w]=\Pi_{\perp} \sum_{k=0}^{N+1} T_{a_{1-k}(\theta, y)} \partial_{x}^{1-k} w+\mathcal{R}_{N, 1}^{\perp}(\theta, y)[w], \\
& X_{2}^{\perp}(\theta)[w, w]=\Pi_{\perp} \sum_{k=0}^{N+1} T_{A_{1-k}(\theta)[w]} \partial_{x}^{1-k} w+\mathcal{R}_{N, 2}^{\perp}(\theta)[w, w],
\end{aligned}
$$

and for any $0 \leq k \leq N+1, a_{1-k}(\theta, y)$ is small of order one, $w \mapsto A_{1-k}(\theta)[w]$ is a linear operator, whereas $w \mapsto \mathcal{R}_{N, 1}^{\perp}(\theta, y)[w]$ is a linear smoothing operator (smoothing of order $N+1$ ), and $w \mapsto \mathcal{R}_{N, 2}^{\perp}(\theta)[w, w]$ is a quadratic smoothing operator (smoothing of order $N+1)$. The term in (1.49), which is small of order three, is the sum of a para-differential vector field of order one and a smoothing vector field.

The second step of our normal form procedure is developed in Sect. 6. Since $\Pi_{\gamma}^{(3)}$ (cf. (1.20)) allows for a loss of derivatives in space, we first need to reduce the terms in the Taylor expansion of the normal component $X^{\perp}$ of $X$, which are linear and quadratic in $w$, to constant coefficients up to smoothing terms - see Sect. 6.1. This regularization procedure is achieved by constructing a transformation which is not canonical, but nevertheless preserves the following important property, needed for the energy estimates: the linearization of $X^{\perp}$ at $w=0$ equals $X_{1}^{\perp}(\theta, y)$ and hence is Hamiltonian. In particular, the diagonal elements of the Fourier matrix representation of the linear operator $X_{1}^{\perp}(\theta, y)$ are purely imaginary,

$$
\left[X_{1}^{\perp}(\theta, y)\right]_{j}^{j} \in \mathrm{i} \mathbb{R}, \quad \forall j \in S^{\perp} .
$$

We remark that in the spirit of [23], one could construct a canonical transformation, but the construction of the one in Sect. 6.1 is technically simpler and due to (1.51) suffices for our purposes. 
We now describe the second step of our normal form procedure in more detail. We begin by normalizing the operator

$$
\Pi_{\perp} T_{a_{1}(\theta, y)} \partial_{x}+\Pi_{\perp} T_{A_{1}(\theta)[w]} \partial_{x}=\Pi_{\perp} T_{a_{1}(\theta, y)+A_{1}(\theta)[w]} \partial_{x}
$$

in the expansion of the vector field $X_{1}^{\perp}(\theta, y)[w]+X_{2}^{\perp}(\theta)[w, w]$ (cf. (1.49), (1.50)). We transform the vector field in (1.49) by the means of the time one flow map $\Phi_{Y}$ of the vector field

$$
Y(\theta, y, w)=\left(0,0, \Pi_{\perp} T_{b(\theta, y)+B(\theta)[w]} \partial_{x}^{-1} w\right)
$$

with $b$ and $B$ given by

$$
\begin{aligned}
b(\theta, y) & :=\frac{1}{3} \partial_{x}^{-1}\left(\left\langle a_{1}(\theta, y)\right\rangle_{x}-a_{1}(\theta, y)\right), \\
B(\theta)[w] & :=\frac{1}{3} \partial_{x}^{-1}\left(\left\langle A_{1}(\theta)[w]\right\rangle_{x}-A_{1}(\theta)[w]\right) .
\end{aligned}
$$

(Recall that for $a \in L^{2}\left(\mathbb{T}_{1}\right),\langle a\rangle_{x}=\int_{0}^{1} a d x$.) Note that $b$ and $B$ satisfy

$$
3 \partial_{x} b(\theta, y)+a_{1}(\theta, y)=\left\langle a_{1}(\theta, y)\right\rangle_{x}, \quad 3 \partial_{x} B(\theta)[w]+A_{1}(\theta)[w]=\left\langle A_{1}(\theta)[w]\right\rangle_{x}
$$

For notational convenience, we denote the transformed vector field also by $X_{1}=$ $\left(X_{1}^{(\theta)}, X_{1}^{(y)}, X_{1}^{\perp}\right)$. We show that $X_{1}^{(y)}$ is small of order three and that $X_{1}^{\perp}(\theta, y, w)$ has the form

$$
\begin{aligned}
& \mathrm{i} \Omega_{\perp} w+\mathcal{D}_{1,1}^{\perp}(\theta, y)[w]+\mathcal{D}_{1,2}^{\perp}(\theta, w)[w]+X_{1,1}^{\perp}(\theta, y)[w] \\
& \quad+X_{1,2}^{\perp}(\theta)[w, w]+\text { term small of order three }
\end{aligned}
$$

with

$$
\begin{aligned}
& \mathcal{D}_{1,1}^{\perp}(\theta, y):=\left\langle a_{1}(\theta, y)\right\rangle_{x} \partial_{x}, \quad \mathcal{D}_{1,2}^{\perp}(\theta, w):=\left\langle A_{1}(\theta)[w]\right\rangle_{x} \partial_{x}, \\
& X_{1,1}^{\perp}(\theta, y)[w]:=\Pi_{\perp} \sum_{k=1}^{N+1} T_{a_{1,1-k}(\theta, y)} \partial_{x}^{1-k} w+\mathcal{R}_{N, 1}^{\perp}(\theta, y)[w], \\
& X_{1,2}^{\perp}(\theta)[w, w]:=\Pi_{\perp} \sum_{k=1}^{N+1} T_{A_{1,1-k}(\theta)[w]} \partial_{x}^{1-k} w+\mathcal{R}_{N, 2}^{\perp}(\theta)[w, w],
\end{aligned}
$$

where for any $1 \leq k \leq N+1, a_{1,1-k}(\theta, y)$ is small of order one and $w \mapsto A_{1,1-k}(\theta)[w]$ is a linear operator. Furthermore, $\mathcal{R}_{N, 1}^{\perp}(\theta, y)$ is a smoothing linear operator and $\mathcal{R}_{N, 2}^{\perp}(\theta)$ is a smoothing bilinear operator. The term in (1.54), which is small of order three, is the sum of a para-differential vector field of order one and a smoothing vector field. We also show that the linear vector field $X_{1,1}^{\perp}(\theta, y)[w]$ in (1.54) satisfies the property (1.51), i.e., $\left[X_{1,1}^{\perp}(\theta, y)\right]_{j}^{j} \in \mathrm{i} \mathbb{R}$ for any $j \in S^{\perp}$, and that the Fourier multiplier $\mathcal{D}_{1,1}^{\perp}(\theta, y)$ is skew-adjoint. By iterating this procedure $N+2$ times, one gets a vector field, which we 
denote by $X_{4}=\left(X_{4}^{(\theta)}, X_{4}^{(y)}, X_{4}^{\perp}\right)$ (cf. Proposition 6.1), with the following properties: $X_{4}^{(y)}$ is small of order three and $X_{4}^{\perp}(\theta, y, w)$ has the form

$$
\begin{array}{r}
\mathrm{i} \Omega_{\perp} w+\mathcal{D}_{4,1}^{\perp}(\theta, y)[w]+\mathcal{D}_{4,2}^{\perp}(\theta, w)[w]+\mathcal{R}_{N, 1}^{\perp}(\theta, y)[w] \\
+\mathcal{R}_{N, 2}^{\perp}(\theta)[w, w]+\text { term small of order three } .
\end{array}
$$

Here $\mathcal{D}_{4,1}^{\perp}(\theta, y)$ and $\mathcal{D}_{4,2}^{\perp}(\theta, w)$ are Fourier multipliers of the form

$$
\mathcal{D}_{4,1}^{\perp}(\theta, y)=\sum_{k=0}^{N+1} \lambda_{1-k}(\theta, y) \partial_{x}^{1-k}, \quad \mathcal{D}_{4,2}^{\perp}(\theta, w):=\sum_{k=0}^{N+1} \Lambda_{1-k}^{\perp}(\theta)[w] \partial_{x}^{1-k},
$$

where for any $0 \leq k \leq N+1, \lambda_{1-k}(\theta, y) \in \mathbb{R}$ is small of order one and $w \mapsto$ $\Lambda_{1-k}^{\perp}(\theta)[w] \in \mathbb{R}$ is a linear operator. The remainder $\mathcal{R}_{N, 1}^{\perp}(\theta, y)$ is a smoothing linear operator and $\mathcal{R}_{N, 2}^{\perp}(\theta)$ is a smoothing bilinear operator. In addition, the Fourier multiplier $\mathcal{D}_{4,1}^{\perp}(\theta, y)$ is skew-adjoint. Moreover we show that

$$
\left[\mathcal{R}_{N, 1}^{\perp}(\theta, y)\right]_{j}^{j} \in \mathrm{i} \mathbb{R}, \quad \forall j \in S^{\perp} .
$$

Since the transformation $\Phi_{Y}$ and the subsequent transformations constructed in the interative procedure are not canonical, the linear operator $\mathcal{D}_{4,2}^{\perp}(\theta, w)$ is not necessarily skew-adjoint. However the leading order term $\Lambda_{1}^{\perp}(\theta)[w] \partial_{x}$ of $\mathcal{D}_{4,2}^{\perp}(\theta, w)$ is skew-adjoint since $\Lambda_{1}^{\perp}(\theta)[w] \in \mathbb{R}$.

In Sect. 6.2 we design a normal form procedure to remove

$$
\sum_{k=1}^{N+1} \Lambda_{1-k}^{\perp}(\theta)[w] \partial_{x}^{1-k}
$$

from $\mathcal{D}_{4,2}^{\perp}(\theta, w)$ which requires to impose first Melnikov conditions on $\omega$ (cf. definition (1.20) of $\Pi_{\gamma}^{(1)}$ ). We transform the vector field $X_{4}$ (cf. (1.55)) by the means of the time one flow map of a vector field, which in view of (1.58) is chosen to be of the form

$$
\left(0,0, \sum_{k=1}^{N+1} \Xi_{1-k}^{\perp}(\theta)[w] \partial_{x}^{1-k} w\right)
$$

where for any $1 \leq k \leq N+1$, the linear functional $w \mapsto \Xi_{1-k}^{\perp}(\theta)[w]$ is a solution of

$$
\omega \cdot \partial_{\theta} \Xi_{1-k}^{\perp}(\theta)[w]-\Xi_{1-k}^{\perp}(\theta)\left[\mathrm{i} \Omega_{\perp} w\right]+\Lambda_{1-k}^{\perp}(\theta)[w]=0 .
$$

The latter equation can be solved if $\omega \in \Pi_{\gamma}^{(1)}$ (first Melnikov conditions). The transformed vector field is denoted by $X_{5}=\left(X_{5}^{(\theta)}, X_{5}^{(y)}, X_{5}^{\perp}\right)$. We show that $X_{5}^{(y)}$ is small of order three and that $X_{5}^{\perp}(\theta, y, w)$ has the form

$$
\begin{aligned}
& \mathrm{i} \Omega_{\perp} w+\mathcal{D}_{5}^{\perp}(\theta, y, w)[w]+\mathcal{R}_{N, 1}^{\perp}(\theta, y)[w]+\mathcal{R}_{N, 2}^{\perp}(\theta)[w, w] \\
& \quad+\text { term small of order three, }
\end{aligned}
$$


where

$$
\mathcal{D}_{5}^{\perp}(\mathfrak{x}):=\mathcal{D}_{4,1}^{\perp}(\theta, y)+\Lambda_{1}^{\perp}(\theta)[w] \partial_{x}
$$

and $\mathcal{R}_{N, 1}^{\perp}, \mathcal{R}_{N, 2}^{\perp}$ are as in (1.55). Clearly, the Fourier multiplier $\mathcal{D} \frac{\perp}{5}(\mathfrak{x})$ is skew-adjoint. Finally in Sect. 6.3 we normalize the term in the Taylor expansion of the $\theta$-component $X_{5}^{(\theta)}$ of $X_{5}$, which is quadratic in $w$, and normalize the smoothing vector fields $\mathcal{R}_{N, 1}^{\perp}$ and $\mathcal{R}_{N, 2}^{\perp}$ in $X_{5}^{\perp}$. Let us explain in more detail how to achieve the latter. We transform the vector field $X_{5}$ by the time one flow map generated by the vector field

$$
\left(0,0, \mathcal{S}_{1}^{\perp}(\theta, y)[w]+\mathcal{S}_{2}^{\perp}(\theta)[w, w]\right)
$$

where $\mathcal{S}_{1}^{\perp}(\theta, y)$ is a smoothing linear operator and $\mathcal{S}_{2}^{\perp}(\theta)$ is a smoothing bilinear operator. They are chosen to be solutions of

$$
-\omega \cdot \partial_{\theta} \mathcal{S}_{1}^{\perp}(\theta, y)+\left[i \Omega_{\perp}, \mathcal{S}_{1}^{\perp}(\theta, y)\right]_{l i n}+\mathcal{R}_{N, 1}^{\perp}(\theta, y)=\mathcal{Z}^{\perp}(y)
$$

and, respectively,

$$
\begin{aligned}
& -\omega \cdot \partial_{\theta} \mathcal{S}_{2}^{\perp}(\theta)[w, w]+\mathrm{i} \Omega_{\perp} \mathcal{S}_{2}^{\perp}(\theta)[w, w]-\mathcal{S}_{2}^{\perp}(\theta)\left(\left[\mathrm{i} \Omega_{\perp} w, w\right]+\left[w, \mathrm{i} \Omega_{\perp} w\right]\right) \\
& \quad+\mathcal{R}_{N, 2}^{\perp}(\theta)[w, w]=0,
\end{aligned}
$$

where

$$
\mathcal{Z}^{\perp}(y):=\operatorname{diag}_{j \in S^{\perp}}\left[\widehat{\mathcal{R}}_{N, 1}^{\perp}(0, y)\right]_{j}^{j}, \quad\left[\widehat{\mathcal{R}}_{N, 1}^{\perp}(0, y)\right]_{j}^{j}:=\frac{1}{(2 \pi)^{S_{+}}} \int_{\mathbb{T}^{S+}}\left[\mathcal{R}_{N, 1}^{\perp}(\theta, y)\right]_{j}^{j} d \theta .
$$

Equation (1.64) can be solved by imposing the second Melnikov conditions on $\omega$, i.e., $\omega \in \Pi_{\gamma}^{(2)}$, and Eq. (1.65) by imposing the third Melnikov conditions, $\omega \in \Pi_{\gamma}^{(3)}$ see Lemma 6.1. Note that in Eq. (1.65), the right hand side vanishes, meaning that the left hand side does not contain any resonant terms. Finally we get a vector field $X_{6}=\left(X_{6}^{(\theta)}, X_{6}^{(y)}, X_{6}^{\perp}\right)$ where $X_{6}^{(y)}$ is small of order three and $X_{6}^{\perp}(\mathfrak{x})$ has the form

$$
X_{6}^{\perp}(\mathfrak{x})=\mathrm{i} \Omega_{\perp} w+\mathcal{D}_{5}^{\perp}(\mathfrak{x})[w]+\mathcal{Z}^{\perp}(y)[w]+\text { term small of order three } .
$$

By the property (1.57) and the definition (1.66) of $\mathcal{Z}^{\perp}(y)$, it follows that $\mathcal{Z}^{\perp}(y)$ and hence $\mathcal{D}_{5}^{\perp}(\mathfrak{x})+\mathcal{Z}^{\perp}(y)$ are skew-adjoint Fourier multiplier. Finally one shows that $X_{6}^{\perp}$ in (1.67) has the form stated in (1.47).

Related work. Prior to our work, no results have been obtained on the long time asymptotics of the solutions of Hamiltonian perturbations of integrable PDEs such as the $\mathrm{KdV}$ or the nonlinear Schrödinger equation on $\mathbb{T}_{1}$ with initial data close to a periodic multi-soliton of possibly large amplitude. For Hamiltonian perturbations of linear integrable PDEs on $\mathbb{T}_{1}$, which satisfy nonresonance conditions, a by now standard normal form method has been developed allowing to prove the stability of the equilibrium solution $u \equiv 0$ of (Hamiltonian) perturbations for time intervals of large size-see e.g. $[2-4,7,13,17,18,23,37]$ and references therein. More recently, these techniques have been refined so that in specific cases, such results can also be proved for Hamiltonian perturbations of resonant linear integrable PDEs by approximating the perturbed equation by nonlinear integrable systems, satisfying nonresonance conditions-see $[5,13]$ 
for Hamiltonian perturbations of the linear Schrödinger equation and [6] for such perturbations of the Airy equation as well as the linearized Benjamin-Ono equation. We remark that for the Airy equation, the Hamiltonian perturbations considered in [6] are of the form $\partial_{x} \nabla P_{f}$ (cf. (1.6)-(1.7)) with the density $f(u(x))$ not explicitly depending on $x$ and $f(z)$ being analytic in a neighborhood of $z=0$ in $\mathbb{C}$.

Finally, we mention the recent paper [8] where it is proved by KAM methods that many periodic multi-solitons persist under quasi-linear perturbations of the $\mathrm{KdV}$ equation. As in this paper, a key ingredient are the normal form coordinates, constructed in [27].

\section{Para-Differential Calculus}

In this section we review some standard notions and results of the para-differential calculus, needed throughout the paper. For details we refer to [37].

We begin with reviewing the notion of para-product. To this end we need the following

Definition 2.1. A function $\psi \in C^{\infty}(\mathbb{R} \times \mathbb{R})$ is said to be an admissible cut-off function, if there exist $0<\varepsilon^{\prime}<\varepsilon<1$ so that

$$
\begin{aligned}
& \operatorname{supp}(\psi) \subseteq\{(\eta, \xi) \in \mathbb{R} \times \mathbb{R}:|\eta| \leq \varepsilon\langle\xi\rangle\}, \\
& \psi(\eta, \xi)=1, \forall(\eta, \xi) \in \mathbb{R} \times \mathbb{R} \text { with }|\eta| \leq \varepsilon^{\prime}\langle\xi\rangle,
\end{aligned}
$$

and

$$
\left|\partial_{\eta}^{\alpha} \partial_{\xi}^{\beta} \psi(\eta, \xi)\right| \lesssim \alpha, \beta\langle\xi\rangle^{-\alpha-\beta}, \quad \forall(\alpha, \beta) \in \mathbb{Z}_{\geq 0} \times \mathbb{Z}_{\geq 0}
$$

where by $(1.21)\langle\xi\rangle=\max \{1,|\xi|\}$.

Given a cut-off function $\psi$ as in Definition 2.1, the para-product $T_{a} u$ of a function $a \in H^{1}\left(\mathbb{T}_{1}\right)$ with a function $u \in H^{s}\left(\mathbb{T}_{1}\right), s \geq 1$, is defined as

$$
\begin{aligned}
T_{a} u(x) & :=\sigma_{a}(x, D) u(x)=\sum_{\xi \in \mathbb{Z}} \sigma_{a}(x, \xi) \widehat{u}(\xi) e^{\mathrm{i} 2 \pi \xi x}, \\
\sigma_{a}(x, \xi) & :=\sum_{\eta \in \mathbb{Z}} \psi(\eta, \xi) \widehat{a}(\eta) e^{\mathrm{i} 2 \pi \eta x},
\end{aligned}
$$

where $\widehat{a}(\eta)$, also denoted by $a_{\eta}$, is the $\eta$ th Fourier coefficient of $a$,

$$
\widehat{a}(\eta)=\int_{0}^{1} a(x) e^{-\mathrm{i} 2 \pi \eta x} d x .
$$

Lemma 2.1. For any $a \in H^{1}\left(\mathbb{T}_{1}\right)$ and $s \geq 1, T_{a}$ is in $\mathcal{B}\left(H^{s}\left(\mathbb{T}_{1}\right), H^{s}\left(\mathbb{T}_{1}\right)\right)$ and

$$
\left\|T_{a}\right\|_{\mathcal{B}\left(H^{s}, H^{s}\right)} \lesssim s\|a\|_{1}
$$

Furthermore, for any $s \geq 1$, the map $H^{1}\left(\mathbb{T}_{1}\right) \rightarrow \mathcal{B}\left(H^{s}\left(\mathbb{T}_{1}\right), H^{s}\left(\mathbb{T}_{1}\right)\right), a \mapsto T_{a}$, is linear. 
Given two functions $a, u \in H^{s}\left(\mathbb{T}_{1}\right)$ with $s \geq 1$, their product can be split as

$$
a u=T_{a} u+T_{u} a+\mathcal{R}^{(B)}(a, u),
$$

where the remainder $\mathcal{R}^{(B)}(a, u)$ is given by $\mathcal{R}^{(B)}(a, u)(x)=\sum_{\eta, \xi \in \mathbb{Z}} \omega(\eta, \xi) \widehat{a}(\eta) \widehat{u}(\xi) e^{\mathrm{i} 2 \pi(\eta+\xi) x}, \quad \omega(\eta, \xi):=1-\psi(\eta, \xi)-\psi(\xi, \eta)$.

Note that the support $\operatorname{supp}(\omega)$ of $\omega: \mathbb{Z} \times \mathbb{Z} \rightarrow \mathbb{R}$ satisfies

$$
\begin{aligned}
& \left\{(\eta, \xi) \in \mathbb{Z}^{2}: \varepsilon\langle\xi\rangle<|\eta|<\frac{\langle\xi\rangle}{\varepsilon}\right\} \cup\{(0,0)\} \subseteq \operatorname{supp}(\omega) \\
& \subseteq\left\{(\eta, \xi) \in \mathbb{Z}^{2}: \varepsilon^{\prime}\langle\xi\rangle<|\eta|<\frac{\langle\xi\rangle}{\varepsilon^{\prime}}\right\} \cup\{(0,0)\} .
\end{aligned}
$$

The main feature of $\mathcal{R}^{(B)}(a, u)$ is that it is a regularizing bilinear operator in the following sense.

Lemma 2.2. For any $s_{1}, s_{2} \geq 0$,

$$
\mathcal{R}^{(B)}: H^{s_{1}+1}\left(\mathbb{T}_{1}\right) \times H^{s_{2}}\left(\mathbb{T}_{1}\right) \rightarrow H^{s_{1}+s_{2}}\left(\mathbb{T}_{1}\right),(a, u) \mapsto \mathcal{R}^{(B)}(a, u)
$$

is a bilinear map, satisfying

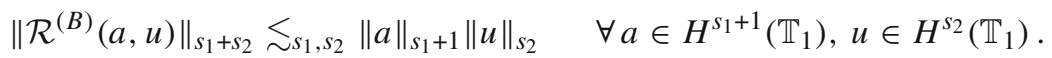

Next, we discuss the standard symbolic calculus for para-differential operators to the extent needed in this paper. It suffices to consider operators of the form

$$
T_{a} \partial_{x}^{m}, \quad a \in H^{1}\left(\mathbb{T}_{1}\right), m \in \mathbb{Z},
$$

where we recall that for any $m \in \mathbb{Z}$, the Fourier multiplier $\partial_{x}^{m}$ is defined by

$$
\partial_{x}^{m}\left[e^{\mathrm{i} 2 \pi j x}\right]:=(\mathrm{i} 2 \pi j)^{m} e^{\mathrm{i} 2 \pi j x}, \quad \forall j \neq 0, \quad \partial_{x}^{m}[1]:=0 .
$$

Alternatively, $\partial_{x}^{m}$ can be written as the pseudo-differential operator $\mathrm{Op}\left((\mathrm{i} 2 \pi \xi)^{m} \chi(\xi)\right)$ with symbol $(\mathrm{i} 2 \pi \xi)^{m} \chi(\xi)$ where $\chi: \mathbb{R} \rightarrow \mathbb{R}$ is a $C^{\infty}$-smooth cut-off function, satisfying

$$
\chi(\xi)=1, \quad \forall|\xi| \geq \frac{2}{3}, \quad \chi(\xi)=0, \quad \forall|\xi| \leq \frac{1}{3}
$$

The symbol of an operator of the form (2.7) is given by

$$
\sigma_{a}(x, \xi)=\sum_{\eta \in \mathbb{Z}} \psi(\eta, \xi) \widehat{a}(\eta)(\mathrm{i} 2 \pi \xi)^{m} e^{\mathrm{i} 2 \pi \eta x} .
$$


Lemma 2.3. Let $a, b \in H^{N+3}\left(\mathbb{T}_{1}\right)$ with $N \in \mathbb{N}$. Then

$$
T_{a} \circ T_{b}=T_{a b}+\mathcal{R}_{N}(a, b)
$$

where for any $s \geq 0$,

$$
\mathcal{R}_{N}: H^{N+3}\left(\mathbb{T}_{1}\right) \times H^{N+3}\left(\mathbb{T}_{1}\right) \rightarrow \mathcal{B}\left(H^{s}\left(\mathbb{T}_{1}\right), H^{s+N+1}\left(\mathbb{T}_{1}\right)\right),(a, b) \mapsto \mathcal{R}_{N}(a, b),
$$

is a bilinear map, satisfying

$$
\left\|\mathcal{R}_{N}(a, b)\right\|_{\mathcal{B}\left(H^{s}, H^{s+N+1}\right)} \lesssim_{s, N}\|a\|_{N+3}\|b\|_{N+3}, \quad \forall a, b \in H^{N+3}\left(\mathbb{T}_{1}\right) .
$$

Lemma 2.4. Let $m \in \mathbb{Z}, N \in \mathbb{N}$. Then there exist an integer $\sigma_{N}>N+m$ and combinatorial constants $\left(K_{n, m}\right)_{1 \leq n \leq N+m}$, with $K_{1, m}=m$ so that for any $a \in H^{\sigma_{N}}\left(\mathbb{T}_{1}\right)$

$$
\partial_{x}^{m} \circ T_{a}=T_{a} \partial_{x}^{m}+\sum_{n=1}^{N+m} K_{n, m} T_{\partial_{x}^{n} a} \partial_{x}^{m-n}+\mathcal{R}_{N, m}(a)
$$

where for any $s \geq 0$, the map

$$
\mathcal{R}_{N, m}: H^{\sigma_{N}}\left(\mathbb{T}_{1}\right) \rightarrow \mathcal{B}\left(H^{s}\left(\mathbb{T}_{1}\right), H^{s+N+1}\left(\mathbb{T}_{1}\right)\right), a \mapsto \mathcal{R}_{N, m}(a)
$$

is linear and satisfies the estimate

$$
\left\|\mathcal{R}_{N, m}(a)\right\|_{\mathcal{B}\left(H^{s}, H^{s+N+1}\right)} \lesssim s, m, N\|a\|_{\sigma_{N}}, \quad \forall a \in H^{\sigma_{N}}\left(\mathbb{T}_{1}\right),
$$

and where we use the customary convention that the sum $\sum_{n=1}^{N+m}$ equals 0 if $N+m<1$.

Combining Lemmas 2.3 and 2.4 yields the following

Lemma 2.5. Let $m, m^{\prime} \in \mathbb{Z}, N \in \mathbb{N}$. Then there exists an integer $\sigma_{N}>N+m$ so that for any $a, b \in H^{\sigma_{N}}\left(\mathbb{T}_{1}\right)$,

$$
T_{a} \partial_{x}^{m} \circ T_{b} \partial_{x}^{m^{\prime}}=T_{a b} \partial_{x}^{m+m^{\prime}}+\sum_{n=1}^{N+m+m^{\prime}} K_{n, m} T_{a \partial_{x}^{n} b} \partial_{x}^{m+m^{\prime}-n}+\mathcal{R}_{N, m, m^{\prime}}(a, b),
$$

where $K_{n, m}$ are the combinatorial constants of Lemma 2.4 and where for any $s \geq 0$, the map

$\mathcal{R}_{N, m, m^{\prime}}: H^{\sigma_{N}}\left(\mathbb{T}_{1}\right) \times H^{\sigma_{N}}\left(\mathbb{T}_{1}\right) \rightarrow \mathcal{B}\left(H^{s}\left(\mathbb{T}_{1}\right), H^{s+N+1}\left(\mathbb{T}_{1}\right)\right),(a, b) \mapsto \mathcal{R}_{N, m, m^{\prime}}(a, b)$ is bilinear and satisfies the estimate

$$
\left\|\mathcal{R}_{N, m, m^{\prime}}(a, b)\right\|_{\mathcal{B}\left(H^{s}, H^{s+N+1}\right)} \lesssim s, m, N\|a\|_{\sigma_{N}}\|b\|_{\sigma_{N}}, \quad \forall a, b \in H^{\sigma_{N}}\left(\mathbb{T}_{1}\right) .
$$

According to Lemma 2.3, in the case $m=0$, a possible choice is $\sigma_{N}=N+3, K_{n, 0}=0$ for $1 \leq n \leq N+m^{\prime}$.

Using that $K_{1, m}=m$, one infers from Lemma 2.5 an expansion of the commutator $\left[T_{a} \partial_{x}^{m}, T_{b} \partial_{x}^{m^{\prime}}\right]_{\text {lin }}$. 
Corollary 2.1 (Commutator expansion). Let $m, m^{\prime} \in \mathbb{Z}, N \in \mathbb{N}$. Then there exists $\sigma_{N}>N+m+m^{\prime}$ so that for any $a, b \in H^{\sigma_{N}}\left(\mathbb{T}_{1}\right),\left[T_{a} \partial_{x}^{m}, T_{b} \partial_{x}^{m^{\prime}}\right]_{\text {lin }}$ has an expansion of the form

$$
T_{m a \partial_{x} b-m^{\prime} b \partial_{x} a} \partial_{x}^{m+m^{\prime}-1}+\sum_{n=2}^{N+m+m^{\prime}}\left(K_{n, m} T_{a \partial_{x}^{n} b}-K_{n, m^{\prime}} T_{b \partial_{x}^{n} a}\right) \partial_{x}^{m+m^{\prime}-n}+\mathcal{R}_{N, m, m^{\prime}}^{\mathcal{C}}(a, b)
$$

where for any $s \geq 0$, the map

$\mathcal{R}_{N, m, m^{\prime}}^{\mathcal{C}}: H^{\sigma_{N}}\left(\mathbb{T}_{1}\right) \times H^{\sigma_{N}}\left(\mathbb{T}_{1}\right) \rightarrow \mathcal{B}\left(H^{s}\left(\mathbb{T}_{1}\right), H^{s+N+1}\left(\mathbb{T}_{1}\right)\right),(a, b) \mapsto \mathcal{R}_{N, m, m^{\prime}}^{\mathcal{C}}(a, b)$ is bilinear and satisfies

$$
\left\|\mathcal{R}_{N, m, m^{\prime}}^{\mathcal{C}}(a, b)\right\|_{\mathcal{B}\left(H^{s}, H^{s+N+1}\right)} \lesssim_{s, m, m^{\prime}, N}\|a\|_{\sigma_{N}}\|b\|_{\sigma_{N}}, \quad \forall a, b \in H^{\sigma_{N}}\left(\mathbb{T}_{1}\right)
$$

According to Lemma 2.3, in the case $m=0, m^{\prime}=0,\left[T_{a}, T_{b}\right]_{\text {lin }}=\mathcal{R}_{N}(a, b)-$ $\mathcal{R}_{N}(b, a)$. Hence a possible choice is $\sigma_{N}=N+3, K_{n, 0}=0$ for $1 \leq n \leq N$.

Finally, we discuss the adjoint $T_{a}^{\top}$ of $T_{a}$ with respect to the standard $L^{2}$-inner product.

Lemma 2.6. Let $a \in H^{N+1}\left(\mathbb{T}_{1}\right)$ with $N \in \mathbb{N}$. Then $T_{a}^{\top}=T_{a}+\mathcal{R}_{\top}(a)$ where for any $s \geq 0$, the map

$$
\mathcal{R}_{\top}: H^{N+1}\left(\mathbb{T}_{1}\right) \rightarrow \mathcal{B}\left(H^{s}\left(\mathbb{T}_{1}\right), H^{s+N+1}\left(\mathbb{T}_{1}\right)\right), a \mapsto \mathcal{R}_{\top}(a),
$$

is linear and for any $a \in H^{N+1}\left(\mathbb{T}_{1}\right)$ satisfies $\left\|\mathcal{R}_{\top}(a)\right\|_{\mathcal{B}\left(H^{s}, H^{s+N+1}\right)} \lesssim_{s, N}\|a\|_{N+1}$.

Combining Lemmas 2.4 and 2.6 yields the following

Corollary 2.2. Let $m \in \mathbb{Z}, N \in \mathbb{N}$. Then there exists an integer $\sigma_{N}>N+m$ so that for any $a \in H^{\sigma_{N}}\left(\mathbb{T}_{1}\right),\left(T_{a} \partial_{x}^{m}\right)^{\top}$ admits the expansion

$$
\left(T_{a} \partial_{x}^{m}\right)^{\top}=(-1)^{m} T_{a} \partial_{x}^{m}+(-1)^{m} \sum_{n=1}^{N+m} K_{n, m} T_{\partial_{x}^{n} a} \partial_{x}^{m-n}+\mathcal{R}_{\top, N, m}(a),
$$

where $K_{n, m}$ are the combinatorial constants of Lemma 2.4, and where for any $s \geq 0$, the map

$$
\mathcal{R}_{\top, N, m}: H^{\sigma_{N}}\left(\mathbb{T}_{1}\right) \rightarrow \mathcal{B}\left(H^{s}\left(\mathbb{T}_{1}\right), H^{s+N+1}\left(\mathbb{T}_{1}\right)\right), a \mapsto \mathcal{R}_{\top, N, m}(a),
$$

is linear and for any $a \in H^{\sigma_{N}}\left(\mathbb{T}_{1}\right)$ satisfies $\left\|\mathcal{R}_{\top, N, m}(a)\right\|_{\mathcal{B}\left(H^{s}, H^{s+N+1}\right)} \lesssim s, N\|a\|_{\sigma_{N}}$.

\section{Para-Differential Vector Fields}

In this section we introduce several classes of vector fields, compute the commutators between vector fields from these classes and study their flows. As part of the proof of Theorem 1.1, these vector fields are used to transform equation (1.4) into normal form. 


\subsection{Definitions.}

Definition 3.1 (Para-differential vector fields). Let $N, p \in \mathbb{N}$ and $m \in \mathbb{Z}$. A vector field $X^{\perp}$ in normal direction, defined on a subset of $\mathcal{E}$ and depending on the parameters $\varepsilon$ and $\mu$, is said to be of class $\mathcal{O} B(m, N), X^{\perp} \in \mathcal{O} B(m, N)$, if it is of the form

$$
X^{\perp}(\mathfrak{x})=\Pi_{\perp} \sum_{k=0}^{N+m} T_{a_{m-k}(\mathfrak{x})} \partial_{x}^{m-k} w
$$

and has the following property: there are integers $\sigma_{N}, s_{N} \geq 0$ so that for any $s \geq s_{N}$ there exist $0<\delta \equiv \delta(s, N)<1$ and $0<\varepsilon_{0} \equiv \varepsilon_{0}(s, N)<1$ so that for any $0 \leq k \leq N+m$

$$
a_{m-k}: \mathcal{V}^{s+\sigma_{N}}(\delta) \times\left[0, \varepsilon_{0}\right] \rightarrow H^{s}\left(\mathbb{T}_{1}\right),(\mathfrak{x}, \varepsilon) \mapsto a_{m-k}(\mathfrak{x}) \equiv a_{m-k}(\mathfrak{x}, \varepsilon)
$$

is $C^{\infty}$-smooth and together with each of its derivatives bounded. $X^{\perp}$ is said to be of class $\mathcal{O B}^{p}(m, N)$ if it is in $\mathcal{O B}(m, N)$ and in addition, the functions $a_{m-k}$ are small of order $p-1$.

Remark 3.1. (i) If $N+m<0$ in (3.1), the sum is defined to be the zero vector field. As a consequence, $\mathcal{O B}(m, N)=\{0\}$ if $N+m<0$. Throughout the paper, the same convention holds for any sum of terms, indexed by an empty set, and for any of the used classes of vector fields.

(ii) We point out that the bounds are uniform in the parameter $\mu$, but no regularity assumptions with respect to $\mu$ are required. Throughout the paper, the same convention holds.

Definition 3.2 (Fourier multiplier vector fields). Let $N, p \in \mathbb{N}$ and $m \in \mathbb{Z}$. A vector field $\mathcal{M}^{\perp}$ in normal direction, defined on a subset of $\mathcal{E}$ and depending on the parameters $\varepsilon$ and $\mu$, is said to be of class $\mathcal{O} F(m, N), \mathcal{M}^{\perp} \in \mathcal{O} F(m, N)$, if it is of the form

$$
\mathcal{M}^{\perp}(\mathfrak{x})=\sum_{k=0}^{N+m} \lambda_{m-k}(\mathfrak{x}) \partial_{x}^{m-k} w
$$

and has the following property: there exist an integer $\sigma_{N} \geq 0,0<\delta \equiv \delta(N)<1$, and $0<\varepsilon_{0} \equiv \varepsilon_{0}(N)<1$ so that for any $0 \leq k \leq N+m$,

$$
\lambda_{m-k}: \mathcal{V}^{\sigma_{N}}(\delta) \times\left[0, \varepsilon_{0}\right] \rightarrow \mathbb{R},(\mathfrak{x}, \varepsilon) \mapsto \lambda_{m-k}(\mathfrak{x}) \equiv \lambda_{m-k}(\mathfrak{x}, \varepsilon)
$$

is $C^{\infty}$-smooth and together with each of its derivatives bounded. $\mathcal{M}^{\perp}$ is said to be of class $\mathcal{O F}^{p}(m, N)$ if it is in $\mathcal{O F}(m, N)$ and in addition, the functions $\lambda_{m-k}$ are small of order $p-1$.

Definition 3.3 (Smoothing vector fields). Let $N, p \in \mathbb{N}$. A vector field $\mathcal{R}$, defined on a subset of $\mathcal{E}$ and depending on the parameters $\varepsilon$ and $\mu$, is said to be of class $\mathcal{O S}(N), \mathcal{R} \in$ $\mathcal{O S}(N)$, if there exist $s_{N} \geq 0$ so that for any $s \geq s_{N}$, there exist $0<\delta \equiv \delta(s, N)<1$ and $0<\varepsilon_{0} \equiv \varepsilon_{0}(s, N)<1$ with the property that

$$
\mathcal{R}: \mathcal{V}^{s}(\delta) \times\left[0, \varepsilon_{0}\right] \rightarrow E_{s+N+1},(\mathfrak{x}, \varepsilon) \mapsto \mathcal{R}(\mathfrak{x}) \equiv \mathcal{R}(\mathfrak{x}, \varepsilon)
$$

is $C^{\infty}$-smooth and together with each of its dervatives bounded. $\mathcal{R}$ is said to be of class $\mathcal{O S}^{p}(N)$ if it is in $\mathcal{O S}(N)$ and in addition is small of order $p$. 
Remark 3.2. For notational convenience, in the sequel, we refer to a function, which is $C^{\infty}$-smooth and together with each of its derivatives bounded, as a function which is $C^{\infty}$-smooth and bounded.

Next we introduce special classes of vector fields which are small of order 2 with respect to $y, w, \varepsilon$.

Definition 3.4. Let $N \in \mathbb{N}$ and $m \in \mathbb{Z}$.

(i) Assume that $X^{\perp}(\mathfrak{x})=\Pi_{\perp} \sum_{k=0}^{m+N} T_{a_{m-k}(\mathfrak{x})} \partial_{x}^{m-k} w$ is of class $\mathcal{O B}^{2}(m, N)$.

(i1) $X^{\perp}$ is said to be of class $\mathcal{O B}_{w}^{2}(m, N)$ if it is linear with respect to $w$. As a consequence, for any $0 \leq k \leq m+N$, the coefficient $a_{m-k}$ is small of order one and independent of $w$. More precisely, there is an integer $s_{N} \geq 0$ so that for any $s \geq s_{N}$, there exist $0<\delta \equiv \delta(s, N)<1$ and $\varepsilon_{0} \equiv \varepsilon_{0}(s, N)>0$ with the property that

$$
a_{m-k}: \mathbb{T}^{S_{+}} \times B_{S_{+}}(\delta) \times\left[0, \varepsilon_{0}\right] \rightarrow H^{S}\left(\mathbb{T}_{1}\right),(\theta, y, \varepsilon) \mapsto a_{m-k}(\theta, y) \equiv a_{m-k}(\theta, y, \varepsilon)
$$

is $C^{\infty}$-smooth and bounded (cf. Remark 3.2). In this case, we often write $X^{\perp}(\theta, y)$ $[w]$ instead of $X^{\perp}(\mathfrak{x})$ where

$$
X^{\perp}(\theta, y):=\Pi_{\perp} \sum_{k=0}^{N+m} T_{a_{m-k}(\theta, y)} \partial_{x}^{m-k} .
$$

(i2) $X^{\perp}$ is said be of class $\mathcal{O B}_{w w}^{2}(m, N)$ if it is quadratic with respect to $w$ and independent of $y$. As a consequence, for any $0 \leq k \leq m+N$, the coefficient $a_{m-k}$ is linear with respect to $w$ and independent of $y$. More precisely, there are integers $s_{N} \geq 0, \sigma_{N} \geq 0$ so that for any $s \geq s_{N}$ there exist $0<\delta \equiv \delta(s, N)<1$ and $0<\varepsilon_{0} \equiv \varepsilon_{0}(s, N)<1$ with the property that

$$
a_{m-k}: \mathbb{T}^{S_{+}} \times H_{\perp}^{s+\sigma_{N}} \times\left[0, \varepsilon_{0}\right] \rightarrow H^{s}\left(\mathbb{T}_{1}\right),(\theta, w, \varepsilon) \mapsto a_{m-k}(\theta, w) \equiv A_{m-k}(\theta)[w],
$$

with

$$
A_{m-k}: \mathbb{T}^{S_{+}} \times\left[0, \varepsilon_{0}\right] \rightarrow \mathcal{B}\left(H_{\perp}^{s+\sigma_{N}}\left(\mathbb{T}_{1}\right), H^{S}\left(\mathbb{T}_{1}\right)\right),(\theta, \varepsilon) \mapsto A_{m-k}(\theta) \equiv A_{m-k}(\theta, \varepsilon)
$$

being $C^{\infty}$-smooth and bounded. In this case we often write $X^{\perp}(\theta, w)[w]$ instead of $X^{\perp}(\mathfrak{x})$ where

$$
X^{\perp}(\theta, w)=\Pi_{\perp} \sum_{k=0}^{N+m} T_{A_{m-k}(\theta)[w]} \partial_{x}^{m-k}
$$

(ii) Assume that $\mathcal{M}^{\perp}(\mathfrak{x})=\sum_{k=0}^{N+m} \lambda_{m-k}(\mathfrak{x}) \partial_{x}^{m-k} w$ is of class $\mathcal{O} \mathcal{F}^{2}(m, N)$.

(ii1) $\mathcal{M}^{\perp}$ is said to be of class $\mathcal{O F}_{w}^{2}(m, N)$ if it is linear with respect to $w$. More precisely, there exist $0<\delta \equiv \delta(N)<1$ and $0<\varepsilon_{0} \equiv \varepsilon_{0}(N)<1$ with the property that for any $0 \leq k \leq m+N$,

$\lambda_{m-k}: \mathbb{T}^{S_{+}} \times B_{S_{+}}(\delta) \times\left[0, \varepsilon_{0}\right] \rightarrow \mathbb{R},(\theta, y, \varepsilon) \mapsto \lambda_{m-k}(\theta, y) \equiv \lambda_{m-k}(\theta, y, \varepsilon)$

is $C^{\infty}$-smooth and bounded. 
(ii2) $\mathcal{M}^{\perp}$ is said to be of class $\mathcal{O F}_{w w}^{2}(m, N)$ if it is quadratic with respect to $w$ and independent of $y$. More precisely, there exist an integer $\sigma_{N} \geq 0,0<\varepsilon_{0} \equiv \varepsilon_{0}(N)<$ 1 , and for any $0 \leq k \leq m+N$ a $C^{\infty}$-smooth map

$$
\Lambda_{m-k}: \mathbb{T}^{S_{+}} \times\left[0, \varepsilon_{0}\right] \rightarrow \mathcal{B}\left(H_{\perp}^{\sigma_{N}}\left(\mathbb{T}_{1}\right), \mathbb{R}\right), \theta \mapsto \Lambda_{m-k}(\theta) \equiv \Lambda_{m-k}(\theta, \varepsilon),
$$

so that $\lambda_{m-k}(\mathfrak{x})=\Lambda_{m-k}(\theta)[w]$.

(iii) Assume that $\mathcal{R}$ is a smoothing vector field of class $\mathcal{O S}(N)$.

(iii) $\mathcal{R}$ is said to be of class $\mathcal{O S}_{w}^{2}(N)$ if $\mathcal{R}(\mathfrak{x})$ of the form $\mathfrak{R}(\theta, y)[w]$ with $\mathfrak{R}$ having the following property: there is an integer $s_{N} \geq 0$ so that for any $s \geq s_{N}$, there exist $0<\delta \equiv \delta(s, N)<1$ and $0<\varepsilon_{0} \equiv \varepsilon_{0}(s, N)<1$ with the property that

$$
\begin{aligned}
\mathfrak{R} & \mathbb{T}^{S_{+}} \times B_{S_{+}}(\delta) \times\left[0, \varepsilon_{0}\right] \rightarrow \mathcal{B}\left(H^{S}\left(\mathbb{T}_{1}\right), H^{s+N+1}\left(\mathbb{T}_{1}\right)\right),(\theta, y, \varepsilon) \\
& \mapsto \mathfrak{R}(\theta, y) \equiv \mathfrak{R}(\theta, y ; \varepsilon)
\end{aligned}
$$

is $C^{\infty}$-smooth, bounded, and small of order one. In the sequel, we will also write $\mathcal{R}(\theta, y)[w]$ for $\mathfrak{R}(\theta, y)[w]$.

(iii2) $\mathcal{R}$ is said to be of class $\mathcal{O} \mathcal{S}_{w w}^{2}(N)$ if $\mathcal{R}$ is quadratic with respect to $w$ and independent of $y$. More precisely, $\mathcal{R}(\mathfrak{x})$ is of the form $\mathfrak{R}(\theta)[w, w]$ with $\mathfrak{R}$ having the following property: there is an integer $s_{N} \geq 0$ so that for any $s \geq s_{N}$ there exists $0<\varepsilon_{0} \equiv \varepsilon_{0}(s, N)<1$ with the property that

$$
\mathfrak{R}: \mathbb{T}^{S_{+}} \times\left[0, \varepsilon_{0}\right] \rightarrow \mathcal{B}_{2}\left(H_{\perp}^{s}\left(\mathbb{T}_{1}\right), H_{\perp}^{s+N+1}\left(\mathbb{T}_{1}\right)\right),(\theta, \varepsilon) \mapsto \mathfrak{R}(\theta) \equiv \mathfrak{R}(\theta, \varepsilon)
$$

is $C^{\infty}$-smooth and bounded. In the sequel, we will often write $\mathcal{R}(\theta)[w, w]$ instead of $\Re(\theta)[w, w]$.

Remark 3.3. For any $N \in \mathbb{N}$ and $m \in \mathbb{Z}$, the following inclusions between the classes of vector fields introduced above hold:

$$
\begin{array}{ll}
\mathcal{O} \mathcal{F}(m, N) \subseteq \mathcal{O} \mathcal{B}(m, N), & \mathcal{O F}^{p}(m, N) \subseteq \mathcal{O B}^{p}(m, N), \\
\mathcal{O} \mathcal{F}_{w}^{2}(m, N) \subseteq \mathcal{O B}_{w}^{2}(m, N), & \mathcal{O F}_{w w}^{2}(m, N) \subseteq \mathcal{O B}_{w w}^{2}(m, N) .
\end{array}
$$

These inclusions hold since by (2.1) the operator $T_{\lambda}$ of para-multiplication with any constant $\lambda \in \mathbb{R}$ satisfies $\Pi_{\perp} T_{\lambda}=\lambda \Pi_{\perp}$.

For notational convenience, we will often not distinguish between a vector field $X$ of the form $\left(0,0, X^{\perp}\right)$ and its normal component $X^{\perp}$. Given two vector fields $X$ and $Y$, defined on a subset of $\mathcal{E}$ and depending on the parameters $\varepsilon$ and $\mu$, we write

$$
X=Y+\mathcal{O}_{1}+\cdots+\mathcal{O}_{n}
$$

if for any $1 \leq j \leq n$, there exists a vector field $X_{j} \in \mathcal{O}_{j}$ so that $X=Y+X_{1}+\cdots+X_{n}$. Here $\mathcal{O}_{j}$ denotes any of the classes of vector fields introduced above.

\subsection{Commutators.}

Lemma 3.1 (Commutators I). Let $N$, $p$, and $q$ be in $\mathbb{N}$.

(i) For any smoothing vector fields $\mathcal{R}, \mathcal{Q} \in \mathcal{O S}(N)$, the commutator [R, Q] is also in $\mathcal{O S}(N)$.

(ii) For any vector fields $\mathcal{R} \in \mathcal{O S}^{p}(N)$ and $\mathcal{Q} \in \mathcal{O S}^{q}(N)$, one has $[\mathcal{R}, \mathcal{Q}] \in \mathcal{O S}^{p+q-1}(N)$ 
Proof. The two items follow from Definition 3.3 (smoothing vector fields) and the definition (1.34) of the commutator.

Lemma 3.2 (Commutators II). Let $N, p, q \in \mathbb{N}$ and $m \in \mathbb{Z}$. If $X=\left(0,0, X^{\perp}\right)$ with $X^{\perp} \in \mathcal{O} B(m, N)$ and $\mathcal{R}=\left(\mathcal{R}^{(\theta)}, \mathcal{R}^{(y)}, \mathcal{R}^{\perp}\right) \in \mathcal{O S}(N)$, then

$$
\begin{aligned}
& {\left[\left(0,0, X^{\perp}\right), \mathcal{R}\right]=\left(0,0, \mathcal{C}_{[X, \mathcal{R}]}^{\perp}\right)+\mathcal{R}_{[X, \mathcal{R}]}, \quad \mathcal{C}_{[X, \mathcal{R}]}^{\perp} \in \mathcal{O} \mathcal{B}(m, N),} \\
& \quad \mathcal{R}_{[X, \mathcal{R}]} \in \mathcal{O} \mathcal{S}(N-m) .
\end{aligned}
$$

If $X^{\perp} \in \mathcal{O} B^{p}(m, N)$ and $\mathcal{R} \in \mathcal{O S}^{q}(N)$, then $\mathcal{C}_{[X, \mathcal{R}]}^{\perp} \in \mathcal{O} \mathcal{B}^{p+q-1}(m, N)$ and $\mathcal{R}_{[X, \mathcal{R}]} \in$ $\mathcal{O S}^{p+q-1}(N-m)$.

Proof. By (3.1), $X$ can be written as $X(\mathfrak{x}):=\sum_{k=0}^{N+m} X_{k}(\mathfrak{x})$ where

$$
X_{k}(\mathfrak{x})=\left(0,0, \Pi_{\perp} T_{a_{m-k}(\mathfrak{x})} \partial_{x}^{m-k} w\right), \quad \forall 0 \leq k \leq N+m .
$$

For any $0 \leq k \leq N+m$, the commutator $\left[X_{k}, \mathcal{R}\right](\mathfrak{x})=d X_{k}(\mathfrak{x})[\mathcal{R}(\mathfrak{x})]-d \mathcal{R}(\mathfrak{x})\left[X_{k}(\mathfrak{x})\right]$ can be computed as

$\left[X_{k}, \mathcal{R}\right](\mathfrak{x})=\left(0,0, \Pi_{\perp} T_{a_{m-k}(\mathfrak{x})} \partial_{x}^{m-k} \mathcal{R}^{\perp}(\mathfrak{x})\right)+\left(0,0, \Pi_{\perp} T_{d a_{m-k}(\mathfrak{x})[\mathcal{R}(\mathfrak{x})]} \partial_{x}^{m-k} w\right)-d \mathcal{R}(\mathfrak{x})\left[X_{k}(\mathfrak{x})\right]$, where $\mathcal{R}=\left(\mathcal{R}^{(\theta)}, \mathcal{R}^{(y)}, \mathcal{R}^{\perp}\right)$. Note that $d \mathcal{R}(\mathfrak{x})\left[X_{k}(\mathfrak{x})\right] \in \mathcal{O} \mathcal{S}(N-m)$ and that for any $0 \leq k \leq N+m$,

$$
\left(0,0, \Pi_{\perp} T_{a_{m-x}(\mathfrak{x})} \partial_{x}^{m-k} \mathcal{R}^{\perp}(\mathfrak{x})\right) \in \mathcal{O} \mathcal{S}(N-(m-k)) \subseteq \mathcal{O S}(N-m) .
$$

Formula (3.3) then follows by setting $\mathcal{C}_{[X, \mathcal{R}]}^{\perp}(\mathfrak{x}):=\Pi_{\perp} \sum_{k=0}^{m+N} T_{d a_{m-k}(\mathfrak{x})[\mathcal{R}(\mathfrak{x})]} \partial_{x}^{m-k} w$, and

$$
\mathcal{R}_{[X, \mathcal{R}]}(\mathfrak{x}):=\sum_{k=0}^{m+N}\left(0,0, \Pi_{\perp} T_{a_{m-k}(\mathfrak{x})} \partial_{x}^{m-k} \mathcal{R}^{\perp}(\mathfrak{x})\right)-d \mathcal{R}(\mathfrak{x})\left[X_{k}(\mathfrak{x})\right] .
$$

The remaining part of the lemma is proved by using similar arguments.

Lemma 3.3 (Commutators III). Let $N, p, q \in \mathbb{N}, m, m^{\prime} \in \mathbb{Z}$, and let $m_{*}:=\max \{m+$ $\left.m^{\prime}-1, m, m^{\prime}\right\}$. For any $X^{\perp} \in \mathcal{O} \mathcal{B}(m, N)$ and $Y^{\perp} \in \mathcal{O} \mathcal{B}\left(m^{\prime}, N\right)$, one has

$\left[X^{\perp}, Y^{\perp}\right]=\mathcal{C}_{\left[X^{\perp}, Y^{\perp}\right]}^{\perp}+\mathcal{R}_{\left[X^{\perp}, Y^{\perp}\right]}^{\perp}, \quad \mathcal{C}_{\left[X^{\perp}, Y^{\perp}\right]}^{\perp} \in \mathcal{O B}\left(m_{*}, N\right), \quad \mathcal{R}_{\left[X^{\perp}, Y^{\perp}\right]}^{\perp} \in \mathcal{O S}(N)$.

If in fact $X^{\perp} \in \mathcal{O B}^{p}(m, N)$ and $Y^{\perp} \in \mathcal{O B}^{q}\left(m^{\prime}, N\right)$, then

$$
\mathcal{C}_{\left[X^{\perp}, Y^{\perp}\right]}^{\perp} \in \mathcal{O} \mathcal{B}^{p+q-1}\left(m_{*}, N\right), \quad \mathcal{R}_{\left[X^{\perp}, Y^{\perp}\right]}^{\perp} \in \mathcal{O} \mathcal{S}^{p+q-1}(N) .
$$

Proof. By formula (3.1), $X^{\perp} \in \mathcal{O B}(m, N)$ and $Y^{\perp} \in \mathcal{O} \mathcal{B}\left(m^{\prime}, N\right)$ are of the form

$$
X^{\perp}(\mathfrak{x})=\Pi_{\perp} \sum_{k=0}^{N+m} T_{a_{m-k}(\mathfrak{x})} \partial_{x}^{m-k} w, \quad Y^{\perp}(\mathfrak{x})=\Pi_{\perp} \sum_{k=0}^{N+m^{\prime}} T_{b_{m^{\prime}-k}(\mathfrak{x})} \partial_{x}^{m^{\prime}-k} w .
$$

With $X^{\perp}=\sum_{k=0}^{N+m} X_{k}^{\perp}$ and $Y^{\perp}=\sum_{j=0}^{N+m^{\prime}} Y_{j}^{\perp}$ one gets

$$
\left[X^{\perp}, Y^{\perp}\right]=\sum_{k=0}^{N+m} \sum_{j=0}^{N+m^{\prime}}\left[X_{k}^{\perp}, Y_{j}^{\perp}\right]
$$


where

$$
\begin{aligned}
& X_{k}^{\perp}(\mathfrak{x})=\Pi_{\perp} T_{a_{m-k}(\mathfrak{x})} \partial_{x}^{m-k} w, \quad \forall 0 \leq k \leq N+m, \\
& Y_{j}^{\perp}(\mathfrak{x}):=\Pi_{\perp} T_{b_{m^{\prime}-j}(\mathfrak{x})} \partial_{x}^{m^{\prime}-j} w, \quad \forall 0 \leq j \leq N+m^{\prime} .
\end{aligned}
$$

To compute $\left[X_{k}^{\perp}, Y_{j}^{\perp}\right.$ ] for $k, j$ in the corresponding ranges, for notational convenience we let

$$
X_{*}^{\perp}:=X_{k}^{\perp}, \quad Y_{*}^{\perp}:=Y_{j}^{\perp}, \quad a(\mathfrak{x}):=a_{m-k}(\mathfrak{x}), \quad b(\mathfrak{x}):=b_{m^{\prime}-j}(\mathfrak{x}), \quad n:=m-k, \quad n^{\prime}:=m^{\prime}-j .
$$

One computes

$\left[X_{*}^{\perp}, Y_{*}^{\perp}\right]=\left[\Pi_{\perp} T_{a} \partial_{x}^{n}, \Pi_{\perp} T_{b} \partial_{x}^{n^{\prime}}\right]_{l i n} w+\Pi_{\perp} T_{d_{\perp} a(\mathfrak{x})\left[Y_{*}^{\perp}(\mathfrak{x})\right]} \partial_{x}^{n} w-\Pi_{\perp} T_{d_{\perp} b(\mathfrak{x})\left[X_{*}^{\perp}(\mathfrak{x})\right]} \partial_{x}^{n^{\prime}} w$.

Using the formula

$$
\Pi_{\perp} T_{a} \partial_{x}^{n} \circ \Pi_{\perp} T_{b} \partial_{x}^{n^{\prime}}=\Pi_{\perp} \circ\left(T_{a} \partial_{x}^{n} \circ T_{b} \partial_{x}^{n^{\prime}}+T_{a} \partial_{x}^{n} \circ\left(\Pi_{\perp}-\mathrm{Id}\right) T_{b} \partial_{x}^{n^{\prime}}\right),
$$

and the corresponding one for $\Pi_{\perp} T_{b} \partial_{x}^{n^{\prime}} \circ \Pi_{\perp} T_{a} \partial_{x}^{n}$, one obtains $\left[X_{*}^{\perp}, Y_{*}^{\perp}\right]=\mathcal{C}_{1}^{\perp}+\mathcal{R}_{1}^{\perp}$ where

$$
\mathcal{C}_{1}^{\perp}(\mathfrak{x}):=\Pi_{\perp}\left[T_{a} \partial_{x}^{n}, T_{b} \partial_{x}^{n^{\prime}}\right]_{l i n} w+\Pi_{\perp} T_{d_{\perp} a(\mathfrak{x})\left[Y_{*}^{\perp}(\mathfrak{x})\right]} \partial_{x}^{n} w-\Pi_{\perp} T_{d_{\perp} b(\mathfrak{x})\left[X_{*}^{\perp}(\mathfrak{x})\right]} \partial_{x}^{n^{\prime}} w
$$

and

$$
\mathcal{R}_{1}^{\perp}(\mathfrak{x}):=\Pi_{\perp} T_{a(\mathfrak{x})} \partial_{x}^{n} \circ\left(\Pi_{\perp}-\mathrm{Id}\right) T_{b(\mathfrak{x})} \partial_{x}^{n^{\prime}} w-\Pi_{\perp} T_{b(\mathfrak{x})} \partial_{x}^{n^{\prime}} \circ\left(\Pi_{\perp}-\mathrm{Id}\right) T_{a(\mathfrak{x})} \partial_{x}^{n} w .
$$

Since by assumption, there exist integers $s_{N} \geq 0, \sigma_{N} \geq 0$, so that for any $s \geq s_{N}$ there is $0<\delta \equiv \delta(s, N)<1$ and $0<\varepsilon_{0} \equiv \varepsilon_{0}(s, N)<1$ with the property that $a, b: \mathcal{V}^{s+\sigma_{N}}(\delta) \times\left[0, \varepsilon_{0}\right] \rightarrow H^{s}\left(\mathbb{T}_{1}\right)$ are $C^{\infty}$-smooth and bounded, it then follows that

$$
\Pi_{\perp} T_{d_{\perp} a(\mathfrak{x})\left[Y_{*}(\mathfrak{x})\right]} \partial_{x}^{n} w \in \mathcal{O} \mathcal{B}(n, N), \quad \Pi_{\perp} T_{d_{\perp} b(\mathfrak{x})\left[X_{*}(\mathfrak{x})\right]} \partial_{x}^{n^{\prime}} w \in \mathcal{O} \mathcal{B}\left(n^{\prime}, N\right),
$$

and, in view of Corollary 2.1, that

$$
\Pi_{\perp}\left[T_{a} \partial_{x}^{n}, T_{b} \partial_{x}^{n^{\prime}}\right]_{l i n} w=\mathcal{O B}\left(n+n^{\prime}-1, N\right)+\mathcal{O S}(N) .
$$

Furthermore, since $\Pi_{\perp}-$ Id is a smoothing operator, one concludes that $\mathcal{R}_{1}^{\perp} \in \mathcal{O S}(N)$. Altogether, we have proved that $\left[X_{*}^{\perp}, Y_{*}^{\perp}\right]$ is of the form $\mathcal{C}_{\left[X_{*}^{\perp}, Y_{*}^{\perp}\right]}^{\perp}+\mathcal{R}_{\left[X_{*}^{\perp}, Y_{*}^{\perp}\right]}^{\perp}$ where $\mathcal{C}_{\left[X_{*}^{\perp}, Y_{*}^{\perp}\right]}^{\perp} \in \mathcal{O B}\left(n_{*}, N\right), \quad n_{*}=\max \left\{n+n^{\prime}-1, n, n^{\prime}\right\} \leq m_{*}, \quad \mathcal{R}_{\left[X_{*}^{\perp}, Y_{*}^{\perp}\right]}^{\perp} \in \mathcal{O S}(N)$.

If in fact $X_{*}^{\perp} \in \mathcal{O B}^{p}(m, N)$ and $Y_{*}^{\perp} \in \mathcal{O B}^{q}\left(m^{\prime}, N\right)$, then $a$ is small of order $p-1, b$ is small of order $q-1$ and it follows that

$$
\begin{gathered}
\Pi_{\perp} T_{d_{\perp} a(\mathfrak{x})\left[Y_{*}^{\perp}(\mathfrak{x})\right]} \partial_{x}^{n} w \in \mathcal{O B}^{p+q-1}(n, N), \quad \Pi_{\perp} T_{d_{\perp} b(\mathfrak{x})\left[X_{*}^{\perp}(\mathfrak{x})\right]} \partial_{x}^{n^{\prime}} w \in \mathcal{O B}^{p+q-1}\left(n^{\prime}, N\right), \\
\Pi_{\perp}\left[T_{a} \partial_{x}^{n}, T_{b} \partial_{x}^{n^{\prime}}\right]_{l i n} w=\mathcal{O} \mathcal{B}^{p+q-1}\left(n+n^{\prime}-1, N\right)+\mathcal{O} \mathcal{S}^{p+q-1}(N), \quad \mathcal{R}_{1}^{\perp} \in \mathcal{O S}^{p+q-1}(N) .
\end{gathered}
$$

One then infers that $\mathcal{C}_{\left[X_{*}^{\perp}, Y_{*}^{\perp}\right]}^{\perp} \in \mathcal{O} \mathcal{B}^{p+q-1}\left(n_{*}, N\right)$ and $\mathcal{R}_{\left[X_{*}^{\perp}, Y_{*}^{\perp}\right]}^{\perp} \in \mathcal{O} \mathcal{S}^{p+q-1}(N)$.

Lemma 3.4 (Commutators $I V$ ). Let $N, p, q \in \mathbb{N}, m, m^{\prime} \in \mathbb{Z}$, and let $m_{*}:=\max \{m+$ $\left.m^{\prime}-1, m, m^{\prime}\right\}$. 
(i) For any $\mathcal{M}^{\perp} \in \mathcal{O} \mathcal{F}(m, N)$ and $\mathcal{M}^{\prime \perp} \in \mathcal{O} \mathcal{F}\left(m^{\prime}, N\right)$

$$
\left[\mathcal{M}^{\perp}, \mathcal{M}^{\prime \perp}\right] \in \mathcal{O} \mathcal{F}\left(m \vee m^{\prime}, N\right) .
$$

If in fact $\mathcal{M}^{\perp} \in \mathcal{O F}^{p}(m, N)$ and $\mathcal{M}^{\perp} \in \mathcal{O F}^{q}\left(m^{\prime}, N\right)$, then $\left[\mathcal{M}^{\perp}, \mathcal{M}^{\prime \perp}\right] \in$ $\mathcal{O} \mathcal{F}^{p+q-1}\left(m \vee m^{\prime}, N\right)$.

(ii) For any $X^{\perp} \in \mathcal{O} \mathcal{B}(m, N)$ and $\mathcal{M}^{\perp} \in \mathcal{O} \mathcal{F}\left(m^{\prime}, N\right)$,

$$
\begin{aligned}
{\left[X^{\perp}, \mathcal{M}^{\perp}\right] } & =\mathcal{C}_{\left[X^{\perp}, \mathcal{M}^{\perp}\right]}^{\perp}+\mathcal{R}_{\left[X^{\perp}, \mathcal{M}^{\perp}\right]}^{\perp}, \quad \mathcal{C}_{\left[X^{\perp}, \mathcal{M}^{\perp}\right]}^{\perp} \in \mathcal{O B}\left(m_{*}, N\right), \quad \mathcal{R}_{\left[X^{\perp}, \mathcal{M}^{\perp}\right]}^{\perp} \in \mathcal{O S}(N) . \\
\text { If } X^{\perp} & \in \mathcal{O B}^{p}(m, N) \text { and } \mathcal{M}^{\perp} \in \mathcal{O F}^{q}\left(m^{\prime}, N\right), \text { then } \\
& \mathcal{C}_{\left[X^{\perp}, \mathcal{M}^{\perp}\right]}^{\perp} \in \mathcal{O} \mathcal{B}^{p+q-1}\left(m_{*}, N\right), \quad \mathcal{R}_{\left[X^{\perp}, \mathcal{M}^{\perp}\right]}^{\perp} \in \mathcal{O} \mathcal{S}^{p+q-1}(N) .
\end{aligned}
$$

(iii) For any $\mathcal{M}=\left(0,0, \mathcal{M}^{\perp}\right)$ with $\mathcal{M}^{\perp} \in \mathcal{O} \mathcal{F}(m, N)$ and $\mathcal{R}=\left(\mathcal{R}^{(\theta)}, \mathcal{R}^{(y)}, \mathcal{R}^{\perp}\right) \in$ $\mathcal{O S}(N)$

$$
\begin{aligned}
& {[\mathcal{M}, \mathcal{R}]=\left(0,0, \mathcal{C}_{[\mathcal{M}, \mathcal{R}]}^{\perp}\right)+\mathcal{R}_{[\mathcal{M}, \mathcal{R}]}, \quad \mathcal{C}_{[\mathcal{M}, \mathcal{R}]}^{\perp} \in \mathcal{O} \mathcal{F}(m, N), \quad \mathcal{R}_{[\mathcal{M}, \mathcal{R}]} \in \mathcal{O S}(N-m)} \\
& \text { If } \mathcal{M}^{\perp} \in \mathcal{O F}^{p}(m, N) \text { and } \mathcal{R} \in \mathcal{O S}^{q}(N), \text { then } \mathcal{C}_{[\mathcal{M}, \mathcal{R}]}^{\perp} \in \mathcal{O} \mathcal{F}^{p+q-1}(m, N) \text { and } \\
& \mathcal{R}_{[M, \mathcal{R}]} \in \mathcal{O S}^{p+q-1}(N) .
\end{aligned}
$$

Proof. Since the claims of the lemma follow by arguing as in the proofs of Lemma 3.2 and Lemma 3.3, the details of the proofs are omitted.

3.3. Flows of para-differential vector fields. In this subsection we study the flow of para-differential vector fields of the form $Y=\left(0,0, Y^{\perp}\right)$ with

$$
Y^{\perp}(\mathfrak{x})=\Pi_{\perp} T_{a_{m}(\mathfrak{x})} \partial_{x}^{m} w \in \mathcal{O B}^{p}(m, N), \quad N, p \geq 1, m \leq 0 .
$$

By Definition 3.1, there are integers $s_{N} \geq 0, \sigma_{N} \geq 0$ so that for any $s \geq s_{N}$ there exist $0<\delta \equiv \delta(s, N)<1$ and $0<\varepsilon_{0} \equiv \varepsilon_{0}(s, N)<1$ with the property that

$$
a_{m}: \mathcal{V}^{s+\sigma_{N}}(\delta) \times\left[0, \varepsilon_{0}\right] \rightarrow H^{s}\left(\mathbb{T}_{1}\right),(\mathfrak{x}, \varepsilon) \mapsto a_{m}(\mathfrak{x}) \equiv a_{m}(\mathfrak{x}, \varepsilon)
$$

is $C^{\infty}$-smooth and bounded. In the sequel, we will often tacitly increase $s_{N}, \sigma_{N}$ and decrease $\delta \equiv \delta(s, N), \varepsilon_{0} \equiv \varepsilon_{0}(s, N)$, whenever needed.

Denote by $\Phi_{Y}(\tau, \cdot)$ the flow associated with $Y$. By the standard ODE theorem in Banach spaces, for any $s \geq s_{N}$, there exist $0<\delta \equiv \delta(s, N)<1$, and $0<\varepsilon_{0} \equiv$ $\varepsilon_{0}(s, N) \ll \delta$, so that for any $-1 \leq \tau \leq 1$,

$$
\Phi_{Y}(\tau, \cdot) \in C_{b}^{\infty}\left(\mathcal{V}^{s}(\delta) \times\left[0, \varepsilon_{0}\right], \mathcal{V}^{s}(2 \delta)\right) .
$$

It then follows that for $-1 \leq \tau \leq 1$ and $\mathfrak{x} \in \mathcal{V}^{S}(\delta)$, one has $\Phi_{Y}\left(-\tau, \Phi_{Y}(\tau, \mathfrak{x})\right)=\mathfrak{x}$.

Remark 3.4. For notational convenience, $\Phi_{Y}(-\tau, \cdot)$ is referred to as the inverse of $\Phi_{Y}(\tau, \cdot)$ and we write $\Phi_{Y}(\tau, \cdot)^{-1}=\Phi_{Y}(-\tau, \cdot)$. In particular, $\Phi_{Y}(1, \cdot)^{-1}=\Phi_{Y}(-1, \cdot)$. Using our convention of tacitly decreasing $\delta$ and $\varepsilon_{0}$, if needed, $\Phi_{Y}(\tau, \cdot)^{-1}$ is defined for $(\mathfrak{x}, \varepsilon) \in \mathcal{V}^{S}(\delta) \times\left[0, \varepsilon_{0}\right]$. More generally, a similar convention is used for diffeomorphisms between neighborhoods of $\mathbb{T}^{S_{+}} \times 0 \times 0$ in $\mathcal{E}_{s}$ throughout the paper.

The following lemma provides a para-differential expansion of the flow $\Phi_{Y}(\tau, \cdot)$. 
Lemma 3.5. Let $N, p \in \mathbb{N}$ and assume that the normal component $Y^{\perp}$ of $Y=\left(0,0, Y^{\perp}\right)$ satisfies (3.4). Then for any $-1 \leq \tau \leq 1, \Phi_{Y}(\tau, \mathfrak{x})$ admits an expansion of the form

$$
\Phi_{Y}(\tau, \mathfrak{x})=\mathfrak{x}+\left(0,0, \Upsilon^{\perp}(\tau, \mathfrak{x})+\mathcal{R}_{N}^{\perp}(\tau, \mathfrak{x})\right)
$$

where

$$
\Upsilon^{\perp}(\tau, \mathfrak{x})=\Pi_{\perp} \sum_{k=0}^{N+m} T_{b_{m-k}(\tau, \mathfrak{x})} \partial_{x}^{m-k} w \in \mathcal{O B}^{p}(m, N), \quad \mathcal{R}_{N}^{\perp}(\tau, \mathfrak{x}) \in \mathcal{O S}^{2 p-1}(N) .
$$

Proof. The normal component $\Phi_{Y}^{\perp}(\tau, \mathfrak{x})$ of the flow $\Phi_{Y}(\tau, \mathfrak{x})$ satisfies the integral equation

$$
\Phi_{Y}^{\perp}(\tau, \mathfrak{x})=w+\int_{0}^{\tau} Y^{\perp}\left(\Phi_{Y}(t, \mathfrak{x})\right) d t, \quad \forall-1 \leq \tau \leq 1 .
$$

To solve it, we make the ansatz that $\Phi_{Y}^{\perp}(\tau, \mathfrak{x})$ admits an expansion of the form

$$
\Phi_{Y}^{\perp}(\tau, \mathfrak{x})=w+\Upsilon^{\perp}(\tau, \mathfrak{x})+\mathcal{R}_{N}^{\perp}(\tau, \mathfrak{x}), \quad \Upsilon^{\perp}(\tau, \mathfrak{x})=\Pi_{\perp} \sum_{k=0}^{N+m} T_{b_{m-k}(\tau, \mathfrak{x})} \partial_{x}^{m-k} w,
$$

with the property that there exist $s_{N} \geq 0, \sigma_{N} \geq 0$ so that the following holds: for any $s \geq s_{N}$, there exist $0<\delta \equiv \delta(s, N)<1$ and $0<\varepsilon_{0} \equiv \varepsilon_{0}(s, N)<1$ so that for any $-1 \leq \tau \leq 1$ and $0 \leq k \leq N+m$,

$$
\begin{aligned}
& b_{m-k}(\tau, \cdot) \in C_{b}^{\infty}\left(\mathcal{V}^{s+\sigma_{N}}(\delta) \times\left[0, \varepsilon_{0}\right], H^{s}\left(\mathbb{T}_{1}\right)\right), b_{m-k} \text { small of order } p-1, \\
& \mathcal{R}_{N}^{\perp}(\tau, \cdot) \in \mathcal{O S}^{p}(N) .
\end{aligned}
$$

To determine $\left(b_{m-k}\right)_{0 \leq k \leq N+m}$ and $\mathcal{R}_{N}^{\perp}$, in terms of the coefficient $a_{m}$ of $Y^{\perp}$ in (3.4), we compute the expansion of the right hand side of the Eq. (3.6) by substituting the ansatz (3.7) into the integrand $Y^{\perp}\left(\Phi_{Y}(t, \mathfrak{x})\right)$. In view of definition (3.4) of $Y^{\perp}$, one gets for any $-1 \leq t \leq 1$,

$$
\begin{aligned}
Y^{\perp}\left(\Phi_{Y}(t, \mathfrak{x})\right) & =\Pi_{\perp} T_{a_{m}\left(\Phi_{Y}(t, \mathfrak{x})\right)} \partial_{x}^{m} \Phi_{Y}^{\perp}(t, \mathfrak{x}) \\
& =\Pi_{\perp} T_{a_{m}\left(\Phi_{Y}(t, \mathfrak{x})\right)} \partial_{x}^{m}\left(w+\Pi_{\perp} \sum_{k=0}^{N+m} T_{b_{m-k}(t, \mathfrak{x})} \partial_{x}^{m-k} w+\mathcal{R}_{N}^{\perp}(t, \mathfrak{x})\right) .
\end{aligned}
$$

Using that $\Pi_{\perp}-\mathrm{Id}$ is a smoothing operator and that

$$
\Phi_{Y}(\tau, \cdot) \in C_{b}^{\infty}\left(\mathcal{V}^{s}(\delta) \times\left[0, \varepsilon_{0}\right], \mathcal{V}^{s}(2 \delta)\right)
$$

one gets

$$
\begin{aligned}
& \Pi_{\perp} T_{a_{m}\left(\Phi_{Y}(t, \mathfrak{x})\right)} \partial_{x}^{m}\left(\Pi_{\perp}-\mathrm{Id}\right) \sum_{k=0}^{N+m} T_{b_{m-k}(t, \mathfrak{x})} \partial_{x}^{m-k} w \in \mathcal{O S}^{2 p-1}(N) \stackrel{p \geq 1}{\subseteq} \mathcal{O} \mathcal{S}^{p}(N), \\
& \Pi_{\perp} \sum_{k=N+1+2 m}^{N+m} T_{a_{m}\left(\Phi_{Y}(t, \mathfrak{x})\right)} \partial_{x}^{m} T_{b_{m-k}(t, \mathfrak{x})} \partial_{x}^{m-k} w \in \mathcal{O S}^{2 p-1}(N) \stackrel{p \geq 1}{\subseteq} \mathcal{O} \mathcal{S}^{p}(N)
\end{aligned}
$$


where we recall that $m \leq 0$ and that by our convention, a sum of terms over an empty index set equals 0 . Moreover, by increasing $s_{N}, \sigma_{N}$ if needed, it follows that for any $s \geq s_{N}$ and $-1 \leq t \leq 1$, the map $A(t, \mathfrak{x}):=\Pi_{\perp} T_{a_{m}\left(\Phi_{Y}(t, \mathfrak{x})\right)} \partial_{x}^{m}$ satisfies (after decreasing $\delta$ and $\varepsilon_{0}$ if necessary)

$$
A(t, \cdot) \in C_{b}^{\infty}\left(\mathcal{V}^{s+\sigma_{N}}(\delta) \times\left[0, \varepsilon_{0}\right], \mathcal{B}\left(H_{\perp}^{s+N+1}\left(\mathbb{T}_{1}\right)\right)\right)
$$

and hence in view of (3.8),

$$
A(t, \cdot)\left[\mathcal{R}_{N}^{\perp}(t, \cdot)\right] \in \mathcal{O S}^{2 p-1}(N) \stackrel{p \geq 1}{\subseteq} \mathcal{O} \mathcal{S}^{p}(N) .
$$

In view of (3.10)-(3.11), we rewrite (3.9) as

$Y^{\perp}\left(\Phi_{Y}(t, \mathfrak{x})\right)=\Pi_{\perp} T_{a_{m}\left(\Phi_{Y}(t, \mathfrak{x})\right)} \partial_{x}^{m} w+\Pi_{\perp} \sum_{k=0}^{N+2 m} T_{a_{m}\left(\Phi_{Y}(t, \mathfrak{x})\right)} \partial_{x}^{m} T_{b_{m-k}(t, \mathfrak{x})} \partial_{x}^{m-k} w+\mathcal{O} \mathcal{S}^{p}(N)$.

Since $a_{m}$ and $b_{m-k}$ are small of order $p-1$ (cf. (3.8)), it follows from Lemma 2.5 that for any $0 \leq k \leq N+2 m$, the term $T_{a_{m}\left(\Phi_{Y}(t, \mathfrak{x})\right)} \partial_{x}^{m} T_{b_{m-k}(t, \mathfrak{x})} \partial_{x}^{m-k} w$ has an expansion of the form

$$
T_{a_{m}\left(\Phi_{Y}(t, \mathfrak{x})\right) b_{m-k}(t, \mathfrak{x})} \partial_{x}^{2 m-k} w+\sum_{j=1}^{N+2 m-k} K(j, m) T_{a_{m}\left(\Phi_{Y}(t, \mathfrak{x})\right) \partial_{x}^{j} b_{m-k}(t, \mathfrak{x})} \partial_{x}^{2 m-k-j} w+\mathcal{O S}^{2 p-1}(N)
$$

with the constants $K(j, m)$ given as in Lemma 2.5, implying that

$$
\begin{aligned}
& \Pi_{\perp} \sum_{k=0}^{N+2 m} T_{a_{m}\left(\Phi_{Y}(t, \mathfrak{x})\right)} \partial_{x}^{m} T_{b_{m-k}(t, \mathfrak{x})} \partial_{x}^{m-k} w=\Pi_{\perp} \sum_{k=0}^{N+2 m} T_{a_{m}\left(\Phi_{Y}(t, \mathfrak{x})\right) b_{m-k}(t, \mathfrak{x})} \partial_{x}^{2 m-k} w \\
& \quad+\Pi_{\perp} \sum_{k=0}^{N+2 m} \sum_{j=1}^{N+2 m-k} K(j, m) T_{a_{m}\left(\Phi_{Y}(t, \mathfrak{x})\right) \partial_{x}^{j} b_{m-k}(t, \mathfrak{x})} \partial_{x}^{2 m-k-j} w+\mathcal{O S}^{2 p-1}(N) \\
& =\Pi_{\perp} \sum_{i=0}^{N+2 m} T_{g_{2 m-i}(t, \mathfrak{x})} \partial_{x}^{2 m-i} w+\mathcal{O S}^{2 p-1}(N),
\end{aligned}
$$

where $g_{2 m}(t, \mathfrak{x})=a_{m}\left(\Phi_{Y}(t, \mathfrak{x})\right) b_{m}(t, \mathfrak{x})$ and for any $1 \leq i \leq N+2 m$,

$g_{2 m-i}(t, \mathfrak{x})=a_{m}\left(\Phi_{Y}(t, \mathfrak{x})\right) b_{m-i}(t, \mathfrak{x})+\sum_{k=1}^{i-1} K(i-k, m) a_{m}\left(\Phi_{Y}(t, \mathfrak{x})\right) \partial_{x}^{i-k} b_{m-k}(t, \mathfrak{x})$.

Combining (3.6)-(3.15) then yields the following identity,

$$
\begin{aligned}
\Pi_{\perp} \sum_{k=0}^{N+m} T_{b_{m-k}(\tau, \mathfrak{x})} \partial_{x}^{m-k} w & =\Pi_{\perp}\left(\int_{0}^{\tau} T_{a_{m}\left(\Phi_{Y}(t, \mathfrak{x})\right)} d t\right) \partial_{x}^{m} w+\Pi_{\perp}\left(\int_{0}^{\tau} T_{a_{m}\left(\Phi_{Y}(t, \mathfrak{x})\right) b_{m}(t, \mathfrak{x})} d t\right) \partial_{x}^{2 m} w \\
& +\Pi_{\perp} \sum_{i=1}^{N+2 m}\left(\int_{0}^{\tau} T_{g_{2 m-i}(t, \mathfrak{x})} d t\right) \partial_{x}^{2 m-i} w+\mathcal{O} \mathcal{S}^{2 p-1}(N) .
\end{aligned}
$$


Let us first consider the case where $m \leq-1$. We then require that the coefficients $b_{m-k}$, $0 \leq k \leq N+m$, satisfy the following system of equations,

$$
\begin{aligned}
b_{m}(\tau, \mathfrak{x}) & =\int_{0}^{\tau} a_{m}\left(\Phi_{Y}(t, \mathfrak{x})\right) d t, \quad b_{m-k}(\tau, \mathfrak{x})=0, \quad \forall 1 \leq k \leq|m|-1, \\
b_{2 m}(\tau, \mathfrak{x}) & =\int_{0}^{\tau} a_{m}\left(\Phi_{Y}(t, \mathfrak{x})\right) b_{m}(t, \mathfrak{x}) d t, \\
b_{m-k}(\tau, \mathfrak{x}) & =\int_{0}^{\tau} g_{m-k}(t, \mathfrak{x}) d t, \quad \forall|m|+1 \leq k \leq N+2 m .
\end{aligned}
$$

Since for any $|m|+1 \leq k \leq N+2 m, g_{m-k}$ only depends on $b_{m-k^{\prime}}$ with $k^{\prime} \leq k+m \leq k-1$ (cf. (3.15)), the coefficients $b_{m-k}$ are determined inductively in terms of $a_{m}$. One then verifies that the properties of the coefficients $b_{m-k}$, stated in ansatz (3.8), are satisfied. The remainder $\mathcal{R}_{N}^{\perp}$ then satisfies the following integral equation

$$
\mathcal{R}_{N}^{\perp}(\tau, \mathfrak{x})=\mathcal{Q}_{N}^{\perp}(\tau, \mathfrak{x})+\int_{0}^{\tau} A(t, \mathfrak{x})\left[\mathcal{R}_{N}^{\perp}(t, \mathfrak{x})\right] d t,
$$

where $\mathcal{Q}_{N}^{\perp}(\tau, \cdot) \in \mathcal{O} \mathcal{S}^{2 p-1}(N)$ is given by the sum of the two terms in (3.10) and the operator $A(t, \mathfrak{x})$ is defined in (3.11). By increasing $s_{N}$ if needed, it follows that for any $s \geq s_{N}$,

$$
\left\|\mathcal{R}_{N}^{\perp}(\tau, \mathfrak{x})\right\|_{s+N+1} \leq \sup _{\tau \in[-1,1]}\left\|\mathcal{Q}_{N}^{\perp}(\tau, \mathfrak{x})\right\|_{s+N+1}+\int_{0}^{\tau}\|A(t, \mathfrak{x})\|_{\mathcal{B}\left(H_{\perp}^{s+N+1}\left(\mathbb{T}_{1}\right)\right)}\left\|\mathcal{R}_{N}^{\perp}(t, \mathfrak{x})\right\|_{s+N+1} d t
$$

and hence by the Gronwall Lemma, one infers that $\mathcal{R}_{N}^{\perp}$ satisfies

$$
\left\|\mathcal{R}_{N}^{\perp}(\tau, \mathfrak{x})\right\|_{s+N+1} \lesssim s, N \exp \left(\int_{-1}^{1}\|A(t, \mathfrak{x})\|_{\mathcal{B}\left(H_{\perp}^{s+N+1}\left(\mathbb{T}_{1}\right)\right)} d t\right) \sup _{t \in[-1,1]}\left\|\mathcal{Q}_{N}^{\perp}(t, \mathfrak{x})\right\|_{s+N+1}
$$

implying that $\left\|\mathcal{R} \frac{\perp}{N}(\tau, \mathfrak{x})\right\|_{s+N+1} \lesssim_{s, N}\left(\varepsilon+\|y\|+\|w\|_{s}\right)^{2 p-1}$. Similar estimates hold for the derivatives of $\mathcal{R}_{N}^{\perp}$. Altogether we have shown that $\mathcal{R}_{N}^{\perp} \in \mathcal{O S}^{2 p-1}(N)$.

Finally let us consider case $m=0$. We then require that the coefficients $b_{-k}, 0 \leq$ $k \leq N$, satisfy the following system of equations,

$$
\begin{gathered}
b_{0}(\tau, \mathfrak{x})=\int_{0}^{\tau} a_{0}\left(\Phi_{Y}(t, \mathfrak{x})\right) d t+\int_{0}^{\tau} a_{0}\left(\Phi_{Y}(t, \mathfrak{x})\right) b_{0}(t, \mathfrak{x}) d t, \\
b_{-k}(\tau, \mathfrak{x})=\int_{0}^{\tau} g_{-k}(t, \mathfrak{x}) d t, \quad \forall 1 \leq k \leq N .
\end{gathered}
$$

The solution $b_{0}$ then reads $b_{0}(\tau, \mathfrak{x})=e^{\int_{0}^{\tau} a_{0}\left(\Phi_{Y}(t, \mathfrak{x})\right) d t}-1$. The remaining part of the proof then follows as in the case $m \leq-1$.

Lemma 3.6. Let $N, p \in \mathbb{N}$ and let $\Phi_{Y}(\tau, \mathfrak{x})$ denote the flow map considered in Lemma 3.5, corresponding to the vector field $Y=\left(0,0, Y^{\perp}\right)$, with $Y^{\perp}(\mathfrak{x})=\Pi_{\perp} T_{a_{m}(\mathfrak{x})} \partial_{x}^{m} w$ and $m \leq 0$, satisfying (3.4). Then for any $-1 \leq \tau \leq 1, d \Phi_{Y}(\tau, \mathfrak{x})^{-1}[\mathfrak{x}]$ admits an expansion of the form

$$
\left.\left.d \Phi_{Y}(\tau, \mathfrak{x})^{-1}[\mathfrak{x}]=\widehat{\mathfrak{x}}+\left(0,0, \Upsilon^{\perp}(\tau, \mathfrak{x}) \hat{\mathfrak{x}}\right]+\mathcal{R} \frac{\perp}{N}(\tau, \mathfrak{x}) \hat{\mathfrak{x}}\right]\right)
$$




$$
\Upsilon^{\perp}(\tau, \mathfrak{x})[\widehat{\mathfrak{x}}]:=\Pi_{\perp} \sum_{k=0}^{N+m} T_{b_{m-k}(\tau, \mathfrak{x})} \partial_{x}^{m-k}[\widehat{w}]+\Pi_{\perp} \sum_{k=0}^{N+m} T_{\left.B_{m-k}(\tau, \mathfrak{x}) \widehat{\mathfrak{x}}\right]} \partial_{x}^{m-k} w
$$

with the following properties: there exist $s_{N}, \sigma_{N} \geq N$ so that for any $s \geq s_{N}$, there exist $\delta \equiv \delta(s, N)>0$ and $0<\varepsilon_{0} \equiv \varepsilon_{0}(s, N)<1$ so that the following holds: for any $0 \leq k \leq N+m$ and $-1 \leq \tau \leq 1$,

$$
\begin{aligned}
& b_{m-k}(\tau, \cdot) \in C_{b}^{\infty}\left(\mathcal{V}^{s+\sigma_{N}}(\delta) \times\left[0, \varepsilon_{0}\right], H^{s}\left(\mathbb{T}_{1}\right)\right), \\
& B_{m-k}(\tau, \cdot) \in C_{b}^{\infty}\left(\mathcal{V}^{s+\sigma_{N}}(\delta) \times\left[0, \varepsilon_{0}\right], \mathcal{B}\left(E_{s+\sigma_{N}}, H^{s}\left(\mathbb{T}_{1}\right)\right)\right), \\
& \mathcal{R}_{N}^{\perp}(\tau, \cdot) \in C_{b}^{\infty}\left(\mathcal{V}^{s}(\delta) \times\left[0, \varepsilon_{0}\right], \mathcal{B}\left(H^{s}\left(\mathbb{T}_{1}\right), H_{\perp}^{s+N+1}\left(\mathbb{T}_{1}\right)\right)\right)
\end{aligned}
$$

with $b_{m-k}(\tau, \cdot), B_{m-k}(\tau, \cdot)$, and $\mathcal{R}_{N}^{\perp}(\tau, \cdot)$ being small of order $p-1$, and the expansion above holds for any $\mathfrak{x} \in \mathcal{V}^{s+\sigma_{N}}(\delta)$ and $\widehat{\mathfrak{x}} \in E_{S+\sigma_{N}}$.

Proof. First we note that for any $-1 \leq \tau \leq 1, d \Phi_{Y}(\tau, \mathfrak{x})^{-1}=d \Phi_{Y}\left(-\tau, \Phi_{Y}(\tau, \mathfrak{x})\right)$ and that by Lemma 3.5,

$$
\Phi_{Y}(\tau, \mathfrak{x})=\mathfrak{x}+\left(0,0, \Pi_{\perp} \sum_{k=0}^{N+m} T_{b_{m-k}\left(\tau, \mathfrak{x} ; \Phi_{Y}\right)} \partial_{x}^{m-k} w+\mathcal{R}_{N}^{\perp}\left(\tau, \mathfrak{x} ; \Phi_{Y}\right)\right)
$$

with $b_{m-k}\left(\tau, \cdot ; \Phi_{Y}\right) \in C_{b}^{\infty}\left(\mathcal{V}^{s+\sigma_{N}}(\delta) \times\left[0, \varepsilon_{0}\right], H^{s}\left(\mathbb{T}_{1}\right)\right)$ being small of order $p-1$ and $\mathcal{R}_{N}^{\perp}\left(\tau, \cdot ; \Phi_{Y}\right) \in \mathcal{O S}^{p}(N)$. To simplify notation, let $\widetilde{b}_{m-k}(\tau, \mathfrak{x}):=b_{m-k}\left(\tau, \mathfrak{x} ; \Phi_{Y}\right)$ and $\widetilde{\mathcal{R}}_{N}^{\perp}(\tau, \mathfrak{x}):=\mathcal{R}_{N}^{\perp}\left(\tau, \mathfrak{x} ; \Phi_{Y}\right)$. Then the normal component of $d \Phi_{Y}(\tau, \mathfrak{x})^{-1}[\widehat{\mathfrak{x}}]-\widehat{\mathfrak{x}}$ can be computed as follows

$$
\begin{aligned}
& \Pi_{\perp} \sum_{k=0}^{N+m} T_{\widetilde{b}_{m-k}\left(-\tau, \Phi_{Y}(\tau, \mathfrak{x})\right)} \partial_{x}^{m-k} \widehat{w}+\Pi_{\perp} \sum_{k=0}^{N+m} T_{\left.d \widetilde{b}_{m-k}\left(-\tau, \Phi_{Y}(\tau, \mathfrak{x})\right) \widehat{\mathfrak{x}}\right]} \partial_{x}^{m-k} \Phi_{Y}^{\perp}(\tau, \mathfrak{x}) \\
& \quad+d \widetilde{\mathcal{R}}_{N}^{\perp}\left(-\tau, \Phi_{Y}(\tau, \mathfrak{x})\right)[\widehat{\mathfrak{x}}] .
\end{aligned}
$$

By expanding the terms $T_{d \widetilde{b}_{m-k}\left(-\tau, \Phi_{Y}(\tau, \mathfrak{x})\right)[\widehat{x}]} \partial_{x}^{m-k} \Phi_{Y}^{\perp}(\tau, \mathfrak{x})$ with the help of Lemma 2.5, one is led to define $b_{m-k}(\tau, \mathfrak{x}), B_{m-k}(\tau, \mathfrak{x})$, and $\mathcal{R}_{N}^{\perp}(\tau, \mathfrak{x})$ with the claimed properties.

Combining Lemmas 3.5 and 3.6, one obtains an expansion of the pullback of various types of vector fields by the time one flow map $\Phi_{Y}(1, \cdot)$ :

Lemma 3.7. Let $N, p, q \in \mathbb{N}$ and let $\Phi_{Y}(1, \mathfrak{x})$ denote the time one flow map, corresponding to the vector field $Y=\left(0,0, Y^{\perp}\right)$, with $Y^{\perp}(\mathfrak{x})=\Pi_{\perp} T_{a_{m}(\mathfrak{x})} \partial_{x}^{m} w$ and $m \leq 0$, satisfying (3.4) (cf. Lemma 3.5). Then the following holds:

(i) For any $X:=\left(0,0, X^{\perp}\right)$ with $X^{\perp} \in \mathcal{O B}^{q}(n, N)$ and $n \geq 0$, the pullback $\Phi_{Y}^{*} X(\mathfrak{x})=$ $d \Phi_{Y}(1, \mathfrak{x})^{-1} X\left(\Phi_{Y}(1, \mathfrak{x})\right)$ of $X$ by $\Phi_{Y}(1, \cdot)$ admits an expansion of the form

$$
\Phi_{Y}^{*} X(\mathfrak{x})=\left(0,0, X^{\perp}(\mathfrak{x})+\Upsilon^{\perp}(\mathfrak{x})+\mathcal{R}_{N}^{\perp}(\mathfrak{x})\right)
$$

where $\Upsilon^{\perp} \in \mathcal{O B}^{p+q-1}(n, N)$ and $\mathcal{R}_{N}^{\perp} \in \mathcal{O} \mathcal{S}^{p+q-1}(N)$.

(ii) For any $X$ in $\mathcal{O S}^{q}(N)$, the pullback $\Phi_{Y}^{*} X$ of $X$ by $\Phi_{Y}(1, \cdot)$ admits an expansion of the form

$$
\Phi_{Y}^{*} X(\mathfrak{x})=X(\mathfrak{x})+\left(0,0, \Upsilon^{\perp}(\mathfrak{x})\right)+\mathcal{R}_{N}(\mathfrak{x})
$$

where $\Upsilon^{\perp} \in \mathcal{O B}^{p+q-1}(m, N)$ and $\mathcal{R}_{N} \in \mathcal{O S}^{p+q-1}(N)$. 
Proof. We only prove item ( $i$ ) since item ( $i i)$ can be proved by similar arguments. Since by (1.36) with $\tau=1$

$$
\Phi_{Y}^{*} X(\mathfrak{x})=X(\mathfrak{x})+\int_{0}^{1}\left(d \Phi_{Y}(t, \mathfrak{x})\right)^{-1}[X, Y]\left(\Phi_{Y}(t, \mathfrak{x})\right) d t,
$$

we analyze for any $t \in[0,1]$ the vector field

$$
Z(t, \mathfrak{x}):=\left(d \Phi_{Y}(t, \mathfrak{x})\right)^{-1}[X, Y]\left(\Phi_{Y}(t, \mathfrak{x})\right) .
$$

Recall that $Y^{\perp}(\mathfrak{x})=\Pi_{\perp} T_{a_{m}(\mathfrak{x})} \partial_{x}^{m} w \in \mathcal{O B}^{p}(m, N)$. Taking into account that $m_{*}=$ $\max \{n+m-1, m, n\}=n$ ( since $n \geq 0 \geq m$ ), it follows from Lemma 3.3 that $[X, Y]=\left(0,0,\left[X^{\perp}, Y^{\perp}\right]\right)$ satisfies

$\left[X^{\perp}, Y^{\perp}\right]=\mathcal{C}_{\left[X^{\perp}, Y^{\perp}\right]}^{\perp}+\mathcal{R}_{\left[X^{\perp}, Y^{\perp}\right]}^{\perp}, \quad \mathcal{C}_{\left[X^{\perp}, Y^{\perp}\right]}^{\perp} \in \mathcal{O B}^{p+q-1}(n, N), \quad \mathcal{R}_{\left[X^{\perp}, Y^{\perp}\right]}^{\perp} \in \mathcal{O S}^{p+q-1}(N)$.

By Definitions 3.1-3.3, and Lemma 3.5, Lemma 3.6, as well as Lemma 2.5, one obtains

$$
\int_{0}^{1} Z(t, \mathfrak{x}) d t=\left(0,0, \Upsilon^{\perp}(\mathfrak{x})+\mathcal{R}_{N}^{\perp}(\mathfrak{x})\right)
$$

with $\Upsilon^{\perp}(\mathfrak{x}) \in \mathcal{O} \mathcal{B}^{p+q-1}(n, N)$ and $\mathcal{R}_{N}^{\perp}(\mathfrak{x}) \in \mathcal{O} \mathcal{S}^{p+q-1}(N)$.

Next we analyze the pullback $\Phi_{Y}^{*} X_{\mathcal{N}}$ of the Hamiltonian vector field $X_{\mathcal{N}}(\mathfrak{x})$ with $\mathcal{N}$ being the following Hamiltonian in normal form (cf. (4.15)),

$$
\mathcal{N}(\mathfrak{x}):=(\omega+\varepsilon \widehat{\omega}) \cdot y+Q(y)+\frac{1}{2}\left\langle D_{\perp}^{-1} \Omega_{\perp} w, w\right\rangle, \quad \omega \in \Pi, \widehat{\omega} \in \mathbb{R}^{S_{+}},
$$

where the Fourier multipliers $D_{\perp}^{-1}$ and $\Omega_{\perp} \equiv \Omega_{\perp}(\omega)$ are given by (1.42) and $Q$ is assumed to be a map in $C_{b}^{\infty}\left(B_{S_{+}}(\delta) \times\left[0, \varepsilon_{0}\right], \mathbb{R}\right)$ with $Q(0)=0$ and $\nabla_{y} Q(0)=0$. Since $\partial_{x} D_{\perp}^{-1} \Omega_{\perp}=\mathrm{i} \Omega_{\perp}$, the vector field $X_{\mathcal{N}}(\mathfrak{x})$ then reads

$$
X_{\mathcal{N}}(\mathfrak{x})=\left(\begin{array}{c}
-\nabla_{y} \mathcal{N}(\mathfrak{x}) \\
\nabla_{\theta} \mathcal{N}(\mathfrak{x}) \\
\partial_{x} \nabla_{\perp} \mathcal{N}(\mathfrak{x})
\end{array}\right)=\left(\begin{array}{c}
-\omega-\varepsilon \widehat{\omega}-\nabla_{y} Q(y) \\
0 \\
i \Omega_{\perp} w
\end{array}\right)
$$

and its differential is given by

$$
d X_{\mathcal{N}}(\mathfrak{x})=\left(\begin{array}{ccc}
0-d_{y} \nabla_{y} Q(y) & 0 \\
0 & 0 & 0 \\
0 & 0 & \mathrm{i} \Omega_{\perp}
\end{array}\right)
$$

Note that $\mathcal{N}(\mathfrak{x})$ does not depend on $\theta$, but only on $y, w$, and $\varepsilon$. For notational convenience, we will often write $\mathcal{N}(y, w)$ instead of $\mathcal{N}(\mathfrak{x})$. The following result on the expansion of $\mathrm{i} \Omega_{\perp}$ can be found in [27].

Lemma 3.8 ([27, Lemma C.7]). For any $N \in \mathbb{N}$, the Fourier multiplier $\mathrm{i} \Omega_{\perp}$ has an expansion of the form

$$
\mathrm{i} \Omega_{\perp}=-\partial_{x}^{3}+\sum_{k=1}^{N} c_{-k} \partial_{x}^{-k}+\mathcal{R}_{N}^{\perp},
$$

where $c_{-k} \equiv c_{-k}(\omega)$ are real constants, depending only on the parameter $\omega \in \Pi$, and $\mathcal{R}_{N}^{\perp} \equiv \mathcal{R}_{N}^{\perp}(\omega)$ is in $\mathcal{B}\left(H_{\perp}^{s}\left(\mathbb{T}_{1}\right), H_{\perp}^{s+N+1}\left(\mathbb{T}_{1}\right)\right)$ for any $s \in \mathbb{R}$. 
Lemma 3.9. Let $X_{\mathcal{N}}$ be the vector field given by (3.22) and $Y=\left(0,0, Y^{\perp}\right)$ be the vector field with $Y^{\perp}(\mathfrak{x})=\Pi_{\perp} T_{a_{m}(\mathfrak{x})} \partial_{x}^{m} w$ and $m \leq 0$, satisfying (3.4) with $p, N \in \mathbb{N}$. Furthermore let $\Phi_{Y}(1, \mathfrak{x})$ be the time one flow map corresponding to the vector field $Y$ (cf. Lemma 3.5). Then the following holds:

(i) If in addition $Y^{\perp}(\mathfrak{x})=\Pi_{\perp} T_{a_{m}(\mathfrak{x})} \partial_{x}^{m} w$ is in $\mathcal{O B}_{w}^{2}(m, N)$, hence $a_{m}(\mathfrak{x}) \equiv a_{m}(\theta, y)$ independent of $w$, and if $\left\langle a_{m}(\mathfrak{x})\right\rangle_{x}=0$, then $\left[X_{\mathcal{N}}, Y\right]$ is of the form $\left(0,0,\left[X_{\mathcal{N}}, Y\right]^{\perp}\right)$ with $\left[X_{\mathcal{N}}, Y\right]^{\perp} \in \mathcal{O B}^{2}(2+m, N)$ and admits an expansion of the form

$$
\left[X_{\mathcal{N}}, Y\right]^{\perp}(\mathfrak{x})=\Pi_{\perp} T_{-3 \partial_{x} a_{m}(\mathfrak{x})} \partial_{x}^{2+m} w+\mathcal{C}^{\perp}(\mathfrak{x})+\mathcal{R}_{N}^{\perp}(\mathfrak{x})+\mathcal{O} \mathcal{B}^{3}(m, N),
$$

where $\mathcal{C}^{\perp}(\mathfrak{x}) \in \mathcal{O B}_{w}^{2}(1+m, N)$ and $\mathcal{R}_{N}^{\perp}(\mathfrak{x}) \in \mathcal{O S}_{w}^{2}(N)$. Moreover $\mathcal{C}^{\perp}(\mathfrak{x})$ and $\mathcal{R}_{N}^{\perp}(\mathfrak{x})$ are of the form $\mathcal{C}^{\perp}(\mathfrak{x})=\mathcal{C}^{\perp}(\theta, y)[w]$ and, respectively, $\mathcal{R}_{N}^{\perp}(\mathfrak{x})=\mathcal{R}_{N}^{\perp}(\theta, y)[w]$, and the diagonal matrix elements of $\mathcal{C}^{\perp}(\theta, y)$ and $\mathcal{R}_{N}^{\perp}(\theta, y)$ vanish,

$$
\left[\mathcal{C}^{\perp}(\theta, y)\right]_{j}^{j}=0, \quad\left[\mathcal{R}_{N}^{\perp}(\theta, y)\right]_{j}^{j}=0, \quad \forall j \in S^{\perp} .
$$

(ii) If in addition $Y^{\perp}(\mathfrak{x})$ is in $\mathcal{O B}_{w w}^{2}(m, N)$, hence $a_{m}(\mathfrak{x})$ of the form $A_{m}(\theta)[w]$, then $\left[X_{\mathcal{N}}, Y\right](\mathfrak{x})$ is of the form $\left(0,0,\left[X_{\mathcal{N}}^{\perp}, Y^{\perp}\right](\mathfrak{x})\right)$ with $\left[X_{\mathcal{N}}, Y\right]^{\perp} \in \mathcal{O B}^{2}(2+m, N)$ and admits an expansion of the form

$$
\left[X_{\mathcal{N}}, Y\right]^{\perp}(\mathfrak{x})=\Pi_{\perp} T_{-3 \partial_{x} A_{m}(\theta)[w]} \partial_{x}^{2+m} w+\mathcal{C}^{\perp}(\mathfrak{x})+\mathcal{R}_{N}^{\perp}(\mathfrak{x})+\mathcal{O B}^{3}(m, N),
$$

where $\mathcal{C}^{\perp}(\mathfrak{x}) \in \mathcal{O B}_{w w}^{2}(1+m, N)$ and $\mathcal{R}_{N}^{\perp}(\mathfrak{x}) \in \mathcal{O S}_{w w}^{2}(N)$.

Proof. (i) Since $Y^{\perp}(\mathfrak{x})=\Pi_{\perp} T_{a_{m}(\mathfrak{x})} \partial_{x}^{m} w$ is in $\mathcal{O B}_{w}^{2}(m, N), a_{m}$ is independent of $w$ and for any $s \geq s_{N}$,

$$
a_{m} \in C_{b}^{\infty}\left(\mathcal{V}^{s+\sigma_{N}}(\delta) \times\left[0, \varepsilon_{0}\right], H^{s}\left(\mathbb{T}_{1}\right)\right) \text { small of order one. }
$$

For notational convenience, we write $Y^{\perp}(\theta, y)[w]$ instead of $Y^{\perp}(\mathfrak{x})$ (similarly as we write $a_{m}(\theta, y)$ instead of $\left.a_{m}(\mathfrak{x})\right)$. Then

$$
\left[X_{\mathcal{N}}, Y\right](\mathfrak{x})=d X_{\mathcal{N}}(y, w)[Y(\mathfrak{x})]-d Y(\mathfrak{x})\left[X_{\mathcal{N}}(y, w)\right]
$$

can be computed as

$$
\begin{aligned}
& {\left[X_{\mathcal{N}}, Y\right](\mathfrak{x}) \stackrel{(3.22),(3.23)}{=}\left(\begin{array}{ccc}
0 & -d_{y}\left(\nabla_{y} Q(y)\right) & 0 \\
0 & 0 & 0 \\
0 & 0 & \mathrm{i} \Omega_{\perp}
\end{array}\right)\left(\begin{array}{c}
0 \\
0 \\
Y^{\perp}(\mathfrak{x})
\end{array}\right)} \\
& \quad-\left(\begin{array}{ccc}
0 & 0 & 0 \\
0 & 0 & 0 \\
\partial_{\theta} Y^{\perp}(\mathfrak{x}) \partial_{y} Y^{\perp}(\mathfrak{x}) & Y^{\perp}(\theta, y)
\end{array}\right)\left(\begin{array}{c}
-\omega-\varepsilon \widehat{\omega}-\nabla_{y} Q(y) \\
0 \\
\mathrm{i} \Omega_{\perp} w
\end{array}\right)=\left(\begin{array}{c}
0 \\
0 \\
{\left[X_{\mathcal{N}}, Y\right]^{\perp}(\mathfrak{x})}
\end{array}\right)
\end{aligned}
$$

where

$\left[X_{\mathcal{N}}, Y\right]^{\perp}(\mathfrak{x}):=\left(\left[\mathrm{i} \Omega_{\perp}, Y^{\perp}(\theta, y)\right]_{l i n}+(\omega+\varepsilon \widehat{\omega}) \cdot \partial_{\theta} Y^{\perp}(\theta, y)+\nabla_{y} Q(y) \cdot \partial_{\theta} Y^{\perp}(\theta, y)\right)[w]$ 
By (3.21), $\nabla_{y} Q(y)$ is small of order one and hence

$$
\begin{aligned}
& \omega \cdot \partial_{\theta} Y^{\perp}(\theta, y)[w]=\Pi_{\perp} T_{\omega \cdot \partial_{\theta} a_{m}(\theta, y)} \partial_{x}^{m} w \in \mathcal{O B}_{w}^{2}(m, N), \\
& \varepsilon \widehat{\omega} \cdot \partial_{\theta} Y^{\perp}(\theta, y)[w]=\varepsilon \Pi_{\perp} T_{\widehat{\omega} \cdot \partial_{\theta} a_{m}(\theta, y)} \partial_{x}^{m} w \in \mathcal{O B}^{3}(m, N), \\
& \nabla_{y} Q(y) \cdot \partial_{\theta} Y^{\perp}(\theta, y)[w]=\Pi_{\perp} T_{\nabla_{y}} Q(y) \cdot \partial_{\theta} a_{m}(\theta, y) \partial_{x}^{m} w \in \mathcal{O} \mathcal{B}^{3}(m, N) .
\end{aligned}
$$

Furthermore by (3.24), Corollary 2.1, and Lemma 3.8, one sees that

$$
\begin{aligned}
& {\left[\mathrm{i} \Omega_{\perp}, Y^{\perp}(\theta, y)\right]_{l i n} w=\Pi_{\perp} T_{-3 \partial_{x} a_{m}(\theta, y)} \partial_{x}^{2+m} w+\mathcal{C}^{(1)}(\theta, y)[w]+\mathcal{R}_{N}^{\perp}(\theta, y)[w]} \\
& \mathcal{C}^{(1)}(\theta, y)[w] \in \mathcal{O B}_{w}^{2}(1+m, N), \quad \mathcal{R}_{N}^{\perp}(\theta, y)[w] \in \mathcal{O S}_{w}^{2}(N) .
\end{aligned}
$$

Altogether we have shown that

$$
\begin{aligned}
& {\left[X_{\mathcal{N}}, Y\right]^{\perp}(\mathfrak{x})=\Pi_{\perp} T_{-3 \partial_{x} a_{m}(\theta, y)} \partial_{x}^{2+m} w+\mathcal{C}^{\perp}(\theta, y)[w]+\mathcal{R}_{N}^{\perp}(\theta, y)[w]+\mathcal{O B}^{3}(m, N),} \\
& \mathcal{C}^{\perp}(\theta, y)[w]:=\mathcal{C}^{(1)}(\theta, y)[w]+\omega \cdot \partial_{\theta} Y^{\perp}(\theta, y)[w] \in \mathcal{O B}_{w}^{2}(1+m, N) .
\end{aligned}
$$

For any $j \in S^{\perp}$, the diagonal matrix element $\left[\omega \cdot \partial_{\theta} Y^{\perp}(\theta, y)\right]_{j}^{j}$ vanishes,

$$
\left[\omega \cdot \partial_{\theta} Y^{\perp}(\theta, y)\right]_{j}^{j}=\omega \cdot \partial_{\theta}\left\langle a_{m}(\theta, y)\right\rangle_{x}(\mathrm{i} 2 \pi j)^{m}=0,
$$

since by assumption $\left\langle a_{m}(\theta, y)\right\rangle_{x}=0$, and so does the diagonal matrix element $\left[\left[i \Omega_{\perp}, Y^{\perp}(\theta, y)\right]_{l i n}\right]_{j}^{j}$, implying together with (3.26)

$$
\left[\mathcal{C}^{\perp}(\theta, y)\right]_{j}^{j}=0, \quad\left[\mathcal{R}_{N}^{\perp}(\theta, y)\right]_{j}^{j}=0, \quad \forall j \in S^{\perp}
$$

(ii) Since $Y^{\perp}(\mathfrak{x})=\Pi_{\perp} T_{a_{m}(\mathfrak{x})} \partial_{x}^{m} w$ is in $\mathcal{O B}_{w w}^{2}(m, N)$, it follows from Definition 3.4 that $a_{m}(\mathfrak{x})$ is of the form $a_{m}(\mathfrak{x})=A_{m}(\theta)[w]$ and that for any $s \geq s_{N}$,

$$
A_{m} \in C_{b}^{\infty}\left(\mathbb{T}^{S_{+}}, \mathcal{B}\left(H_{\perp}^{s+\sigma_{N}}\left(\mathbb{T}_{1}\right), H^{S}\left(\mathbb{T}_{1}\right)\right) .\right.
$$

For notational convenience, we write $a_{m}(\theta, w)$ instead of $a_{m}(\mathfrak{x})$. Arguing as in (3.25), one sees that $\left[X_{\mathcal{N}}, Y\right](\mathfrak{x})=d X_{\mathcal{N}}(y, w)[Y(\mathfrak{x})]-d Y(\mathfrak{x})\left[X_{\mathcal{N}}(y, w)\right]$ can be computed as

$$
\begin{aligned}
& {\left[X_{\mathcal{N}}, Y\right](\mathfrak{x})=\left(\begin{array}{ccc}
0 & -d_{y}\left(\nabla_{y} Q(y)\right) & 0 \\
0 & 0 & 0 \\
0 & 0 & \mathrm{i} \Omega_{\perp}
\end{array}\right)\left(\begin{array}{c}
0 \\
0 \\
Y^{\perp}(\mathfrak{x})
\end{array}\right)} \\
& -\left(\begin{array}{ccc}
0 & 0 & 0 \\
0 & 0 & 0 \\
d_{\theta} Y^{\perp}(\mathfrak{x}) & 0 & d_{\perp} Y^{\perp}(\mathfrak{x})
\end{array}\right)\left(\begin{array}{c}
-\omega-\varepsilon \widehat{\omega}-\nabla_{y} Q(y) \\
0 \\
\mathrm{i} \Omega_{\perp} w
\end{array}\right)=\left(\begin{array}{c}
0 \\
0 \\
{\left[X_{\mathcal{N}}, Y\right]^{\perp}(\mathfrak{x})}
\end{array}\right)
\end{aligned}
$$

where

$\left[X_{\mathcal{N}}, Y\right]^{\perp}(\mathfrak{x})=\mathrm{i} \Omega_{\perp}\left[Y^{\perp}(\mathfrak{x})\right]-d_{\perp} Y^{\perp}(\mathfrak{x})\left[\mathrm{i} \Omega_{\perp} w\right]+(\omega+\varepsilon \widehat{\omega}) \cdot \partial_{\theta} Y^{\perp}(\mathfrak{x})+\nabla_{y} Q(y) \cdot \partial_{\theta} Y^{\perp}(\mathfrak{x})$ 
Since by (3.21), $\nabla_{y} Q(y)$ is small of order one, one infers that

$$
\begin{aligned}
& \omega \cdot \partial_{\theta} Y^{\perp}(\mathfrak{x})=\Pi_{\perp} T_{\omega \cdot \partial_{\theta} A_{m}(\theta)[w]} \partial_{x}^{m} w \in \mathcal{O B}_{w w}^{2}(m, N), \\
& \varepsilon \widehat{\omega} \cdot \partial_{\theta} Y^{\perp}(\mathfrak{x})=\varepsilon \Pi_{\perp} T_{\widehat{\omega} \cdot \partial_{\theta}} A_{m}(\theta)[w] \\
& \nabla_{x}^{m} w \in \mathcal{O B}^{3}(m, N), \\
& { }_{y} Q(y) \cdot \partial_{\theta} Y^{\perp}(\mathfrak{x})=\Pi_{\perp} T_{\nabla_{y}} Q(y) \cdot \partial_{\theta} A_{m}(\theta)[w] \partial_{x}^{m} w \in \mathcal{O B}^{3}(m, N) .
\end{aligned}
$$

Furthermore, $\mathrm{i} \Omega_{\perp}\left[Y^{\perp}(\mathfrak{x})\right]-d_{\perp} Y(\mathfrak{x})\left[\mathrm{i} \Omega_{\perp} w\right]$ can be computed as

$$
\begin{aligned}
& \mathrm{i} \Omega_{\perp} \Pi_{\perp} T_{A_{m}(\theta)[w]} \partial_{x}^{m} w-\Pi_{\perp} T_{A_{m}(\theta)[w]} \partial_{x}^{m} \mathrm{i} \Omega_{\perp} w-\Pi_{\perp} T_{A_{m}(\theta)\left[\mathrm{i} \Omega_{\perp} w\right]} \partial_{x}^{m} w \\
& =\Pi_{\perp}\left[\mathrm{i} \Omega_{\perp}, T_{A_{m}(\theta)[w]} \partial_{x}^{m}\right]_{l i n} w-\Pi_{\perp} T_{A_{m}(\theta)\left[\mathrm{i} \Omega_{\perp} w\right]} \partial_{x}^{m} w .
\end{aligned}
$$

By (3.27), Corollary 2.1, and Lemma 3.8 one has

$$
\begin{aligned}
& \Pi_{\perp} T_{A_{m}(\theta)\left[i \Omega_{\perp} w\right]} \partial_{x}^{m} w \in \mathcal{O} \mathcal{B}_{w w}^{2}(m, N), \\
& \Pi_{\perp}\left[\mathrm{i} \Omega_{\perp}, T_{A_{m}(\theta)[w]} \partial_{x}^{m}\right]_{l i n} w=\Pi_{\perp} T_{-3 \partial_{x} A_{m}(\theta)[w]} \partial_{x}^{2+m} w+\mathcal{C}^{(1)}(\mathfrak{x})+\mathcal{R}_{N}^{\perp}(\mathfrak{x})+\mathcal{O} \mathcal{B}^{3}(m, N), \\
& \mathcal{C}^{(1)}(\mathfrak{x}) \in \mathcal{O} \mathcal{B}_{w w}^{2}(1+m, N), \quad \mathcal{R}_{N}^{\perp}(\mathfrak{x}) \in \mathcal{O} \mathcal{S}_{w w}^{2}(N) .
\end{aligned}
$$

Altogether, the identities (3.29)-(3.31) yield

$$
\begin{aligned}
& {\left[X_{\mathcal{N}}, Y\right]^{\perp}(\mathfrak{x})=\Pi_{\perp} T_{-3 \partial_{x}} A_{m}(\theta)[w] \partial_{x}^{2+m} w+\mathcal{C}^{\perp}(\mathfrak{x})+\mathcal{R}_{N}^{\perp}(\mathfrak{x})+\mathcal{O} \mathcal{B}^{3}(m, N),} \\
& \mathcal{C}^{\perp}(\mathfrak{x}):=\mathcal{C}^{(1)}(\mathfrak{x})+\Pi_{\perp} T_{\omega \cdot \partial_{\theta}} A_{m}(\theta)[w] \partial_{x}^{m} w-\Pi_{\perp} T_{A_{m}(\theta)\left[i \Omega_{\perp} w\right]} \partial_{x}^{m} w \in \mathcal{O} \mathcal{B}_{w w}^{2}(1+m, N)
\end{aligned}
$$

and hence item $(i i)$ is proved.

Lemma 3.10. Let $X_{\mathcal{N}}$ be the vector field given by (3.22) and let $Y(\mathfrak{x})=\left(0,0, Y^{\perp}(\mathfrak{x})\right)$ where $Y^{\perp}(\mathfrak{x})=\left(0,0, Y_{0}^{\perp}(\mathfrak{x})+Y_{1}^{\perp}(\mathfrak{x})\right)$ and

$$
\begin{gathered}
Y_{0}^{\perp}(\mathfrak{x}) \equiv Y_{0}^{\perp}(\theta, y)[w]=\Pi_{\perp} T_{a_{m}(\theta, y)} \partial_{x}^{m} w \in \mathcal{O B}_{w}^{2}(m, N), \\
Y_{1}^{\perp}(\mathfrak{x})=\Pi_{\perp} T_{A_{m}(\theta)[w]} \partial_{x}^{m} w \in \mathcal{O B}_{w w}^{2}(m, N),
\end{gathered}
$$

with $N \in \mathbb{N}$ and $m \leq 0$. If in addition $\left\langle a_{m}(\theta, y)\right\rangle_{x}=0$, then the pullback $X_{\mathcal{N}, \Phi} \equiv$ $\Phi_{Y}^{*} X_{\mathcal{N}}$ of the vector field $X_{\mathcal{N}}$ by be the time one flow map $\Phi_{Y}(1, \cdot)$ corresponding to $Y$ has an expansion of the form

$$
X_{\mathcal{N}, \Phi}(\mathfrak{x})=\left(-\omega-\varepsilon \widehat{\omega}-\nabla_{y} Q(y), 0, X_{\mathcal{N}, \Phi}^{\perp}(\mathfrak{x})\right)
$$

where

$$
\begin{aligned}
X_{\mathcal{N}, \Phi}^{\perp}(\mathfrak{x})= & \mathrm{i} \Omega_{\perp} w+\Pi_{\perp} T_{-3 \partial_{x}\left(a_{m}(\theta, y)+A_{m}(\theta)[w]\right)} \partial_{x}^{2+m} w+\mathcal{C}_{0}^{\perp}(\theta, y)[w]+\mathcal{C}_{1}^{\perp}(\mathfrak{x}) \\
& +\mathcal{R}_{N, 0}^{\perp}(\theta, y)[w]+\mathcal{R}_{N, 1}^{\perp}(\mathfrak{x})+\mathcal{O B}^{3}(2+m, N)+\mathcal{O S}^{3}(N)
\end{aligned}
$$

and $\mathcal{C}_{0}^{\perp}(\theta, y), \mathcal{R}_{N, 0}^{\perp}(\theta, y)$, and $\mathcal{C}_{1}^{\perp}(\mathfrak{x}), \mathcal{R}_{N, 1}^{\perp}(\mathfrak{x})$ are given by Lemma 3.9. Hence these terms satisfy

$$
\begin{array}{ll}
\mathcal{C}_{0}^{\perp}(\theta, y)[w] \in \mathcal{O} \mathcal{B}_{w}^{2}(1+m, N), & \mathcal{R}_{N, 0}^{\perp}(\theta, y)[w] \in \mathcal{O S}_{w}^{2}(N), \\
\mathcal{C}_{1}^{\perp}(\mathfrak{x}) \in \mathcal{O B}_{w w}^{2}(1+m, N), & \mathcal{R}_{N, 1}^{\perp}(\mathfrak{x}) \in \mathcal{O S}_{w w}^{2}(N),
\end{array}
$$

and the diagonal matrix elements of $\mathcal{C}_{0}^{\perp}(\theta, y)$ and $\mathcal{R}_{N, 0}^{\perp}(\theta, y)$ vanish,

$$
\left[\mathcal{C}_{0}^{\perp}(\theta, y)\right]_{j}^{j}=0, \quad\left[\mathcal{R}_{N, 0}^{\perp}(\theta, y)\right]_{j}^{j}=0, \quad \forall j \in S^{\perp} .
$$


Proof. By (1.36), $X_{\mathcal{N}, \Phi}$ can be expanded as

$$
\begin{aligned}
X_{\mathcal{N}, \Phi} & =\Phi_{Y}^{*} X_{\mathcal{N}}=X_{\mathcal{N}}+\left[X_{\mathcal{N}}, Y\right]+Z \\
Z(\mathfrak{x}) & :=\int_{0}^{1}(1-t)\left(d \Phi_{Y}(t, \mathfrak{x})\right)^{-1}\left[\left[X_{\mathcal{N}}, Y\right], Y\right]\left(\Phi_{Y}(t, \mathfrak{x})\right) d t .
\end{aligned}
$$

By Lemma 3.9, one has $\left[X_{\mathcal{N}}, Y\right]=\left(0,0,\left[X_{\mathcal{N}}, Y\right]^{\perp}\right)$ with $\left[X_{\mathcal{N}}, Y\right]^{\perp} \in \mathcal{O B}^{2}(2+m, N)$ given by

$$
\begin{aligned}
& \Pi_{\perp} T_{-3 \partial_{x}\left(a_{m}(\theta, y)+A_{m}(\theta)[w]\right)} \partial_{x}^{2+m} w+\mathcal{C}_{0}^{\perp}(\theta, y)[w] \\
& +\mathcal{R}_{N, 0}^{\perp}(\theta, y)[w]+\mathcal{C}_{1}^{\perp}(\mathfrak{x})+\mathcal{R}_{N, 1}^{\perp}(\mathfrak{x})+\mathcal{O B}^{3}(m, N),
\end{aligned}
$$

where $\mathcal{C}_{0}^{\perp}(\theta, y), \mathcal{R}_{N, 0}^{\perp}(\theta, y)$, and $\mathcal{C}_{1}^{\perp}(\mathfrak{x}), \mathcal{R}_{N, 1}^{\perp}(\mathfrak{x})$ are given as in Lemma 3.9. In particular, the diagonal matrix elements of $\mathcal{C}_{0}^{\perp}(\theta, y)$ and $\mathcal{R}_{N, 0}^{\perp}(\theta, y)$ vanish. Furthermore, by Lemmata 3.2, 3.3, one infers that

$$
\left[\left[X_{\mathcal{N}}, Y\right], Y\right](\mathfrak{x})=\left(0,0, \mathcal{C}_{2}^{\perp}(\mathfrak{x})+\mathcal{R}_{N, 2}^{\perp}(\mathfrak{x})\right), \quad \mathcal{C}_{2}^{\perp} \in \mathcal{O B}^{2}(2+m, N), \quad \mathcal{R}_{N, 2}^{\perp} \in \mathcal{O S}^{3}(N),
$$

and hence concludes by Lemma 3.7 that

$$
Z(\mathfrak{x})=\left(0,0, \mathcal{C}_{3}^{\perp}(\mathfrak{x})+\mathcal{R}_{N, 3}^{\perp}(\mathfrak{x})\right), \quad \mathcal{C}_{3}^{\perp} \in \mathcal{O B}^{3}(2+m, N), \quad \mathcal{R}_{N, 3}^{\perp} \in \mathcal{O S}^{3}(N) .
$$

The claimed statement then follows by (3.33)-(3.35).

3.4. Flows of Fourier multiplier vector fields and smoothing vector fields. In this subsection we discuss additional properties of Fourier multiplier vector fields and smooth vector fields and their flows, needed in Sect. 6.2.

We begin by considering the flows corresponding to Fourier multiplier vector fields. Let $\mathcal{M}$ be a vector field of the form $\left(0,0, \mathcal{M}^{\perp}\right)$ with $\mathcal{M}^{\perp} \in \mathcal{O F}^{p}(0, N)$ and $N, p \in \mathbb{N}$ (cf. Definition 3.2). Then $\mathcal{M}^{\perp}(\mathfrak{x})$ has an expansion of the form $\mathcal{M}^{\perp}(\mathfrak{x})=\sum_{k=0}^{N} \lambda_{-k}(\mathfrak{x}) \partial_{x}^{-k}$ $w$ with the property that there exist $\sigma_{N} \geq 0,0<\delta \equiv \delta(N)<1$, and $0<\varepsilon_{0} \equiv \varepsilon_{0}(N)<$ 1 , so that for any $0 \leq k \leq N$,

$$
\lambda_{-k}: \mathcal{V}^{\sigma_{N}}(\delta) \times\left[0, \varepsilon_{0}\right] \rightarrow \mathbb{R},(\mathfrak{x}, \varepsilon) \mapsto \lambda_{m-k}(\mathfrak{x}) \equiv \lambda_{-k}(\mathfrak{x}, \varepsilon)
$$

is $C^{\infty}$-smooth and bounded. We denote by $\Phi_{\mathcal{M}}(\tau, \cdot)$ the flow corresponding to the vector field $\mathcal{M}$. By the standard ODE theorem in Banach spaces, there exist $s_{N} \geq 0$ so that for any $s \geq s_{N}$, there exist $0<\delta \equiv \delta(s, N)<1$, and $0<\varepsilon_{0} \equiv \varepsilon_{0}(s, N) \ll \delta$, so that

$$
\Phi_{\mathcal{M}}(\tau, \cdot) \in C_{b}^{\infty}\left(\mathcal{V}^{s}(\delta) \times\left[0, \varepsilon_{0}\right], \mathcal{V}^{s}(2 \delta)\right), \quad \forall-1 \leq \tau \leq 1
$$

The following lemma can be proved arguing as in the proof of Lemma 3.5 (actually, the proof is simpler). 
Lemma 3.11. For any $\tau \in[-1,1]$, the flow map $\Phi_{\mathcal{M}}(\tau, \cdot)$ admits an expansion of the form

$$
\Phi_{\mathcal{M}}(\tau, \mathfrak{x})=\mathfrak{x}+\left(0,0, \Upsilon^{\perp}(\tau, \mathfrak{x})+\mathcal{R}_{N}^{\perp}(\tau, \mathfrak{x})\right)
$$

where $\Upsilon^{\perp}(\tau, \cdot) \in \mathcal{O F}^{p}(0, N)$ and $\mathcal{R}_{N}^{\perp} \in \mathcal{O S}^{2 p-1}(N)$.

The following lemma can be proved arguing as in the proof of Lemma 3.6.

Lemma 3.12. Let $\Phi_{\mathcal{M}}(\tau, \mathfrak{x})$ denote the flow map considered in Lemma 3.11, corresponding to the vector field $\mathcal{M}=\left(0,0, \mathcal{M}^{\perp}\right)$ with $\mathcal{M}^{\perp} \in \mathcal{O F}^{p}(0, N)$ and $N, p \in \mathbb{N}$. Then $d \Phi_{\mathcal{M}}(\tau, \mathfrak{x})^{-1}[\widehat{\mathfrak{x}}]$ admits an expansion of the form

$$
\begin{aligned}
& \left.d \Phi_{\mathcal{M}}(\tau, \mathfrak{x})^{-1}[\widehat{\mathfrak{x}}]=\widehat{\mathfrak{x}}+\left(0,0, \Upsilon^{\perp}(\tau, \mathfrak{x}) \widehat{\mathfrak{x}}\right]+\mathcal{R}_{N}^{\perp}(\tau, \mathfrak{x})[\widehat{\mathfrak{x}}]\right), \\
& \left.\Upsilon^{\perp}(\tau, \mathfrak{x}) \hat{\mathfrak{x}}\right]:=\sum_{k=0}^{N} \lambda_{-k}(\tau, \mathfrak{x}) \partial_{x}^{-k} \widehat{w}+\sum_{k=0}^{N} \eta_{-k}(\tau, \mathfrak{x})[\widehat{\mathfrak{x}}] \partial_{x}^{-k} w,
\end{aligned}
$$

with the following properties: there exist $s_{N}, \sigma_{N} \geq N$ so that for any $s \geq s_{N}$, there exist $0<\delta \equiv \delta(s, N)<1$ and $0<\varepsilon_{0} \equiv \varepsilon_{0}(s, N)<1$ so that the following holds: for any $0 \leq k \leq N$ and $-1 \leq \tau \leq 1$,

$$
\begin{aligned}
& \lambda_{-k} \in C_{b}^{\infty}\left(\mathcal{V}^{\sigma_{N}}(\delta) \times\left[0, \varepsilon_{0}\right], \mathbb{R}\right), \quad \eta_{-k} \in C_{b}^{\infty}\left(\mathcal{V}^{\sigma_{N}}(\delta) \times\left[0, \varepsilon_{0}\right], \mathcal{B}\left(E_{\sigma_{N}}, \mathbb{R}\right)\right), \\
& \mathcal{R}_{N}^{\perp} \in C_{b}^{\infty}\left(\mathcal{V}^{s}(\delta) \times\left[0, \varepsilon_{0}\right], \mathcal{B}\left(H_{\perp}^{s}\left(\mathbb{T}_{1}\right), H_{\perp}^{s+N+1}\left(\mathbb{T}_{1}\right)\right)\right),
\end{aligned}
$$

and $\lambda_{-k}(\tau, \cdot), \eta_{-k}(\tau, \cdot)$, and $\mathcal{R}_{N}^{\perp}(\tau, \cdot)$ are small of order $p-1$.

The following lemma can be proved arguing as in the proof of Lemma 3.7.

Lemma 3.13. Let $\Phi_{\mathcal{M}}(1, \mathfrak{x})$ denote the time one flow map considered in Lemma 3.11, corresponding to the vector field $\mathcal{M}=\left(0,0, \mathcal{M}^{\perp}\right)$, with $\mathcal{M}^{\perp} \in \mathcal{O} \mathcal{F}^{p}(0, N)$ and $N$, $p \in \mathbb{N}$. Then the following holds:

(i) For any $X:=\left(0,0, X^{\perp}\right)$ with $X^{\perp} \in \mathcal{O B}^{q}(n, N)$ and $q \geq 1, n \geq 0$, the pullback $\Phi_{\mathcal{M}}^{*} X$ of $X$ by $\Phi_{\mathcal{M}}(1, \cdot)$ admits an expansion of the form

$$
\begin{aligned}
& \Phi_{\mathcal{M}}^{*} X(\mathfrak{x})=\left(0,0, X^{\perp}(\mathfrak{x})+\Upsilon^{\perp}(\mathfrak{x})+\mathcal{R}_{N}^{\perp}(\mathfrak{x})\right), \\
& \Upsilon^{\perp} \in \mathcal{O B}^{p+q-1}(n, N), \quad \mathcal{R}_{N}^{\perp} \in \mathcal{O} \mathcal{S}^{p+q-1}(N) .
\end{aligned}
$$

(ii) For any $\mathcal{M}_{1}=\left(0,0, \mathcal{M}_{1}^{\perp}\right)$ with $\mathcal{M}_{1}^{\perp} \in \mathcal{O F}^{q}(n, N)$ and $q \geq 1, n \geq 0$, the pullback $\Phi_{\mathcal{M}}^{*} \mathcal{M}_{1}$ of $\mathcal{M}_{1}$ by $\Phi_{\mathcal{M}}(1, \cdot)$ admits an expansion of the form

$$
\begin{gathered}
\Phi_{\mathcal{M}}^{*} \mathcal{M}_{1}(\mathfrak{x})=\left(0,0, \mathcal{M}_{1}^{\perp}(\mathfrak{x})+\Upsilon^{\perp}(\mathfrak{x})+\mathcal{R}_{N}^{\perp}(\mathfrak{x})\right), \\
\Upsilon^{\perp} \in \mathcal{O} \mathcal{F}^{p+q-1}(n, N), \quad \mathcal{R}_{N}^{\perp} \in \mathcal{O} \mathcal{S}^{p+q-1}(N) .
\end{gathered}
$$

(iii) For any $X \in \mathcal{O S}^{q}(N)$, the pullback $\Phi_{\mathcal{M}}^{*} X$ of $X$ by $\Phi_{\mathcal{M}}(1, \cdot)$ admits an expansion of the form

$$
\Phi_{\mathcal{M}}^{*} X(\mathfrak{x})=X(\mathfrak{x})+\left(0,0, \Upsilon^{\perp}(\mathfrak{x})\right)+\mathcal{R}_{N}(\mathfrak{x})
$$

where $\Upsilon^{\perp} \in \mathcal{O} \mathcal{F}^{p+q-1}(0, N)$ and $\mathcal{R}_{N} \in \mathcal{O} \mathcal{S}^{p+q-1}(N)$. 
Next we consider $\mathcal{M}:=\left(0,0, \mathcal{M}^{\perp}\right)$ with $\mathcal{M}^{\perp} \in \mathcal{O F}_{w w}^{2}(0, N)$ and $N \in \mathbb{N}$ (cf. Definition 3.4-(ii2)), i.e., $\mathcal{M}^{\perp}(\mathfrak{x})=\mathcal{M}^{\perp}(\theta, w)[w]$ with $\mathcal{M}^{\perp}(\theta, w)=\sum_{k=0}^{N} \Lambda_{-k}(\theta)[w] \partial_{x}^{-k}$ where, for some integer $\sigma_{N} \geq 0$ and some $0<\varepsilon_{0} \equiv \varepsilon_{0}(N)<1$,

$$
\Lambda_{-k}: \mathbb{T}^{S_{+}} \times\left[0, \varepsilon_{0}\right] \rightarrow \mathcal{B}\left(H_{\perp}^{\sigma_{N}}\left(\mathbb{T}_{1}\right), \mathbb{R}\right), \theta \mapsto \Lambda_{-k}(\theta) \equiv \Lambda_{-k}(\theta, \varepsilon), \quad 0 \leq k \leq N
$$

are $C^{\infty}$-smooth. To obtain an expansion of the pullback $\Phi_{\mathcal{M}}^{*} X_{\mathcal{N}}$ of the vector field $X_{\mathcal{N}}$, defined in (3.22), by $\Phi_{\mathcal{M}}(1, \cdot)$, we first need to compute the one of the commutator $\left[X_{\mathcal{N}}, \mathcal{M}\right]$.

Lemma 3.14. The commutator $\left[X_{\mathcal{N}}, \mathcal{M}\right](\mathfrak{x})$ admits an expansion of the form

$$
\left[X_{\mathcal{N}}, \mathcal{M}\right](\mathfrak{x})=\left(0,0, \omega \cdot \partial_{\theta}\left(\mathcal{M}^{\perp}(\theta, w)[w]\right)-\mathcal{M}^{\perp}\left(\theta, \mathrm{i} \Omega_{\perp} w\right)[w]+\mathcal{O F}^{3}(0, N)\right)
$$

Proof. By (3.37) the differential of $\mathcal{M}$ can be computed as

$$
d \mathcal{M}(\mathfrak{x}) \widehat{\mathfrak{x}}]=\left(0,0, \mathcal{M}^{\perp}(\theta, w)[\widehat{w}]+\mathcal{M}^{\perp}(\theta, \widehat{w})[w]+d_{\theta}(\mathcal{M}(\theta, w)[w])[\widehat{\theta}]\right) .
$$

By (3.22), (3.23), the commutator

$$
\left[X_{\mathcal{N}}, \mathcal{M}\right](\mathfrak{x})=d X_{\mathcal{N}}(y, w)[\mathcal{M}(\mathfrak{x})]-d \mathcal{M}(\mathfrak{x})\left[X_{\mathcal{N}}(y, w)\right]
$$

is given by

$$
\begin{aligned}
& {\left[X_{\mathcal{N}}, \mathcal{M}\right](\mathfrak{x})=\left(0,0, \mathrm{i} \Omega_{\perp} \mathcal{M}^{\perp}(\theta, w)[w]\right)} \\
& \quad-\left(0,0, \mathcal{M}^{\perp}(\theta, w)\left[\mathrm{i} \Omega_{\perp} w\right]+\mathcal{M}^{\perp}\left(\theta, \mathrm{i} \Omega_{\perp} w\right)[w]-d_{\theta}\left(\mathcal{M}^{\perp}(\theta, w)[w]\right)\left[\omega+\varepsilon \widehat{\omega}+\nabla_{y} Q(y)\right]\right) \\
& \quad=\left(0,0,\left[\mathrm{i} \Omega_{\perp}, \mathcal{M}^{\perp}(\theta, w)\right]_{l i n} w-\mathcal{M}^{\perp}\left(\theta, \mathrm{i} \Omega_{\perp} w\right)[w]\right. \\
& \left.\quad+\left(\omega+\varepsilon \widehat{\omega}+\nabla_{y} Q(y)\right) \cdot \partial_{\theta}\left(\mathcal{M}^{\perp}(\theta, w)[w]\right)\right) .
\end{aligned}
$$

Since $\mathcal{M}^{\perp}(\theta, w)$ and $\mathrm{i} \Omega_{\perp}$ are both Fourier multipliers, the linear commutator $\left[\mathrm{i} \Omega_{\perp}\right.$, $\left.\mathcal{M}^{\perp}(\theta, w)\right]_{\text {lin }}$ vanishes. The lemma then follows in view of the fact that

$$
\begin{aligned}
& \left(\varepsilon \widehat{\omega}+\nabla_{y} Q(y)\right) \cdot \partial_{\theta}\left(\mathcal{M}^{\perp}(\theta, w)[w]\right) \\
& \left.=\sum_{k=0}^{N}\left(\varepsilon \widehat{\omega}+\nabla_{y} Q(y)\right) \cdot \partial_{\theta}\right)\left(\Lambda_{-k}(\theta)[w]\right) \partial_{x}^{-k}[w] \\
& \quad \in \mathcal{O F}^{3}(0, N) .
\end{aligned}
$$

Lemma 3.15. The pullback $\Phi_{\mathcal{M}}^{*} X_{\mathcal{N}}$ of the vector field $X_{\mathcal{N}}$ by $\Phi_{\mathcal{M}}(1, \cdot)$ with $\mathcal{M}$ given by (3.37) admits an expansion of the form

$$
\Phi_{\mathcal{M}}^{*} X_{\mathcal{N}}(\mathfrak{x})=\left(\begin{array}{c}
-\omega-\varepsilon \widehat{\omega}-\nabla_{y} Q(y) \\
0 \\
i \Omega_{\perp} w+\omega \cdot \partial_{\theta}\left(\mathcal{M}^{\perp}(\theta, w)[w]\right)-\mathcal{M}^{\perp}\left(\theta, i \Omega_{\perp} w\right)[w]+\mathcal{O} \mathcal{F}^{3}(0, N)+\mathcal{O S}^{3}(N)
\end{array}\right) .
$$


Proof. We argue as in the proof of Lemma 3.10. By (1.36), $\Phi_{\mathcal{M}}^{*} X_{\mathcal{N}}$ can be expanded as

$$
\begin{aligned}
\Phi_{\mathcal{M}}^{*} X_{\mathcal{N}} & =X_{\mathcal{N}}+\left[X_{\mathcal{N}}, \mathcal{M}\right]+Z, \\
Z(\mathfrak{x}) & :=\int_{0}^{1}(1-\tau)\left[d \Phi_{\mathcal{M}}(\tau, \mathfrak{x})\right]^{-1}\left[\left[X_{\mathcal{N}}, \mathcal{M}\right], \mathcal{M}\right]\left(\Phi_{\mathcal{M}}(\tau, \mathfrak{x})\right) d \tau .
\end{aligned}
$$

The claimed statement then follows by applying Lemmata 3.4, 3.11, 3.12, 3.14.

Finally, we consider smoothing vector fields. Given a smoothing vector field $\mathcal{Q} \in$ $\mathcal{O S}^{p}(N)$ with $N, p \in \mathbb{N}$ (cf. Definition 3.3), we denote by $\Phi_{\mathcal{Q}}(\tau, \cdot)$ the flow corresponding to the vector field $\mathcal{Q}$. By the standard ODE theorem in Banach spaces, there exists $s_{N} \geq 0$ so that for $s \geq s_{N}$, there exist $0<\delta \equiv \delta(s, N)<1$ and $0<\varepsilon_{0} \equiv \varepsilon_{0}(s, N) \ll \delta$, so that

$$
\begin{aligned}
& \Phi_{\mathcal{Q}}(\tau, \cdot) \in C_{b}^{\infty}\left(\mathcal{V}^{s}(\delta) \times\left[0, \varepsilon_{0}\right], \mathcal{V}^{s}(2 \delta)\right), \\
& \Phi_{\mathcal{Q}}(\tau, \cdot)-\text { Id } \text { small of order } p, \quad \forall-1 \leq \tau \leq 1 .
\end{aligned}
$$

Lemma 3.16. Let $\mathcal{Q} \in \mathcal{O S}^{p}(N)$ with $N, p \in \mathbb{N}$. For any $-1 \leq \tau \leq 1$, the following holds.

(i) The flow map $\Phi_{\mathcal{Q}}(\tau, \cdot)$ admits an expansion of the form

$$
\Phi_{\mathcal{Q}}(\tau, \mathfrak{x})=\mathfrak{x}+\mathcal{R}_{N}(\tau, \mathfrak{x}), \quad \mathcal{R}_{N}(\tau, \cdot) \in \mathcal{O S}^{p}(N) .
$$

(ii) The map $d \Phi_{\mathcal{S}}(\tau, \mathfrak{x})^{-1}$ admits an expansion of the form

$$
d \Phi_{\mathcal{Q}}(\tau, \mathfrak{x})^{-1}[\widehat{\mathfrak{x}}]=\widehat{\mathfrak{x}}+\mathcal{R}_{N}(\tau, \mathfrak{x})[\widehat{\mathfrak{x}}]
$$

where there exists $s_{N} \geq 0$ so that for any $s \geq s_{N}$ there are $0<\delta \equiv \delta(s, N)<1$ and $0<\varepsilon_{0} \equiv \varepsilon_{0}(s, N)<1$ such that

$$
\mathcal{R}_{N}(\tau, \cdot) \in C_{b}^{\infty}\left(\mathcal{V}^{s}(\delta) \times\left[0, \varepsilon_{0}\right], \mathcal{B}\left(E_{s}, E_{s+N+1}\right)\right), \quad \forall-1 \leq \tau \leq 1 .
$$

Proof. To prove item ( $i$ ) one uses the Volterra integral equation (cf. (3.6)) and (3.38) (cf. proof of Lemma 3.5). To prove item (ii), one argues as in the proof of Lemma 3.6, using the identity $d \Phi_{\mathcal{Q}}(\tau, \mathfrak{x})^{-1}=d \Phi_{\mathcal{Q}}\left(-\tau, \Phi_{\mathcal{Q}}(\tau, \mathfrak{x})\right),-1 \leq \tau \leq 1$ (cf. Remark 3.4).

Lemma 3.17. For any $\mathcal{Q} \in \mathcal{O S}^{p}(N)$ with $N, p \in \mathbb{N}$, the following holds:

(i) For any $X:=\left(0,0, X^{\perp}\right)$ with $X^{\perp} \in \mathcal{O B}^{q}(m, N)$ and $m \in \mathbb{Z}, q \in \mathbb{N}$, the pullback $\Phi_{\mathcal{Q}^{*}}^{*}$ of $X$ by $\Phi_{\mathcal{Q}}(1, \cdot)$ admits an expansion of the form

$$
\Phi_{\mathcal{Q}^{*}}^{*}(\mathfrak{x})=\left(0,0, X^{\perp}(\mathfrak{x})+\Upsilon^{\perp}(\mathfrak{x})+\mathcal{R}_{N}^{\perp}\right), \quad \Upsilon^{\perp} \in \mathcal{O B}^{p+q-1}(m, N), \quad \mathcal{R}_{N}^{\perp} \in \mathcal{O S}^{p+q-1}(N) .
$$

(ii) For any $\mathcal{M}:=\left(0,0, \mathcal{M}^{\perp}\right)$ with $\mathcal{M}^{\perp} \in \mathcal{O F}^{q}(m, N)$ and $m \in \mathbb{Z}, q \in \mathbb{N}$, the pullback $\Phi_{\mathcal{Q}}^{*} \mathcal{M}$ of $\mathcal{M}$ by $\Phi_{\mathcal{Q}}(1, \cdot)$ admits an expansion of the form

$$
\begin{aligned}
& \Phi_{\mathcal{Q}^{*}} \mathcal{M}(\mathfrak{x})=\left(0,0, \mathcal{M}^{\perp}(\mathfrak{x})+\Upsilon^{\perp}(\mathfrak{x})+\mathcal{R}_{N}^{\perp}(\mathfrak{x})\right), \\
& \Upsilon^{\perp} \in \mathcal{O} \mathcal{F}^{p+q-1}(m, N), \quad \mathcal{R}_{N}^{\perp} \in \mathcal{O} \mathcal{S}^{p+q-1}(N) .
\end{aligned}
$$

(iii) For any $\mathcal{Q}_{1} \in \mathcal{O} \mathcal{S}^{q}(N)$ with $q \in \mathbb{N}$, the pullback $\Phi_{\mathcal{Q}}^{*} \mathcal{Q}_{1}$ of $\mathcal{Q}_{1}$ by $\Phi_{\mathcal{Q}}(1, \cdot)$ admits an expansion of the form $\Phi_{\mathcal{Q}}^{*} \mathcal{Q}_{1}=\mathcal{Q}_{1}+\mathcal{O S}^{p+q-1}(N)$. 
Proof. (i) By (1.36), $\Phi_{\mathcal{Q}}^{*} X(\mathfrak{x})$ can be expanded as

$$
\Phi_{\mathcal{Q}}^{*} X(\mathfrak{x})=X(\mathfrak{x})+Z, \quad Z:=\int_{0}^{1} d \Phi_{\mathcal{Q}}(t, \mathfrak{x})^{-1}[X, \mathcal{Q}]\left(\Phi_{\mathcal{Q}}(t, \mathfrak{x})\right) d t .
$$

By applying Lemma 3.2, one gets that

$$
[X, \mathcal{Q}]=\left(0,0, \Upsilon^{\perp}+\mathcal{R}_{[X, \mathcal{Q}]}^{\perp}\right), \quad \Upsilon^{\perp} \in \mathcal{O B}^{p+q-1}(m, N), \quad \mathcal{R}_{[X, \mathcal{Q}]}^{\perp} \in \mathcal{O S}^{p+q-1}(N+m) .
$$

Item $(i)$ then follows by the definition of $Z$, the property (3.38), and Lemma 3.16. Items (ii) and (iii) can be proved similarly, using in addition Lemma 3.1 and Lemma 3.4.

We now consider a smoothing vector field $\mathcal{Q} \in \mathcal{O}(N), N \in \mathbb{N}$, of the form $\mathcal{Q}:=$ $\mathcal{Q}_{0}+\mathcal{Q}_{1}$ where

$$
\begin{array}{ll}
\mathcal{Q}_{0}:=\left(0,0, \mathcal{Q}_{0}^{\perp}\right), & \mathcal{Q}_{0}^{\perp}(\mathfrak{x}) \equiv \mathcal{Q}_{0}^{\perp}(\theta, y)[w] \in \mathcal{O S}_{w}^{2}(N), \\
\mathcal{Q}_{1}:=\left(\mathcal{F}_{1}, 0, \mathcal{Q}_{1}^{\perp}\right), & \mathcal{Q}_{1}^{\perp}(\mathfrak{x}) \equiv \mathcal{Q}_{1}^{\perp}(\theta)[w, w] \in \mathcal{O S}_{w w}^{2}(N),
\end{array}
$$

(cf. Definition 3.4(iii) for the definitions of $\mathcal{O S}_{w}^{2}(N)$ and $\mathcal{O} \mathcal{S}_{w w}^{2}(N)$ ) and where for some $\sigma_{N} \geq 0$ and $0<\varepsilon_{0} \equiv \varepsilon_{0}(N)<1, \mathcal{F}_{1}$ has the form

$$
\mathcal{F}_{1}(\theta, w):=F_{1}(\theta)[w, w], \quad F_{1} \in C^{\infty}\left(\mathbb{T}^{S_{+}} \times\left[0, \varepsilon_{0}\right], \mathcal{B}_{2}\left(H_{\perp}^{\sigma_{N}}\left(\mathbb{T}_{1}\right), \mathbb{R}^{S_{+}}\right)\right),
$$

(cf. (1.39) for the definition $\mathcal{B}_{2}\left(H_{\perp}^{\sigma_{N}}\left(\mathbb{T}_{1}\right), \mathbb{R}^{S_{+}}\right)$). In the next lemma we compute an expansion of $\Phi_{\mathcal{Q}}^{*} X_{\mathcal{N}}$ where $X_{\mathcal{N}}$ is the normal form vector field defined in (3.22).

Lemma 3.18. For $\mathcal{Q}=\mathcal{Q}_{0}+\mathcal{Q}_{1}$ as in (3.39), the following holds.

(i) The commutator $\left[X_{\mathcal{N}}, \mathcal{Q}_{0}\right] \in \mathcal{O S}^{2}(N-3)$ has the form $\Upsilon^{(1)}+\mathcal{O S}^{3}(N)$ where

$$
\Upsilon^{(1)}(\mathfrak{x})=\left(0,0,\left(\left[\mathrm{i} \Omega_{\perp}, \mathcal{Q}_{0}^{\perp}(\theta, y)\right]_{l i n}+\omega \cdot \partial_{\theta} \mathcal{Q}_{0}^{\perp}(\theta, y)\right)[w]\right) .
$$

(ii) The commutator $\left[X_{\mathcal{N}}, \mathcal{Q}_{1}\right] \in \mathcal{O S}^{2}(N-3)$ has the form $\Upsilon^{(2)}+\mathcal{O S}^{3}(N)$ where

$$
\Upsilon^{(2)}(\mathfrak{x})=\left(\begin{array}{c}
\omega \cdot \partial_{\theta} F_{1}(\theta)[w, w]-F_{1}(\theta)\left[i \Omega_{\perp} w, w\right]-F_{1}(\theta)\left[w, \mathrm{i} \Omega_{\perp} w\right] \\
0 \\
\mathrm{i} \Omega_{\perp} \mathcal{Q}_{1}^{\perp}(\theta)[w, w]-\mathcal{Q}_{1}^{\perp}(\theta)\left[\mathrm{i} \Omega_{\perp} w, w\right]-\mathcal{Q}_{1}^{\perp}(\theta)\left[w, \mathrm{i} \Omega_{\perp} w\right]+\omega \cdot \partial_{\theta} \mathcal{Q}_{1}^{\perp}(\theta)[w, w]
\end{array}\right)
$$

(iii) The pullback $\Phi_{\mathcal{Q}^{*}} X_{\mathcal{N}}$ is of the form $X_{\mathcal{N}}+\Upsilon^{(1)}+\Upsilon^{(2)}+\mathcal{O S} \mathcal{S}^{3}(N)$ with $\Upsilon^{(1)}$ given by item (i) and $\Upsilon^{(2)}$ given by item (ii).

Proof. ( $i$ ) Arguing as in the proof of Lemma 3.9(i) (cf. (3.25)), one sees that $\left[X_{\mathcal{N}}, \mathcal{Q}_{0}\right](\mathfrak{x})$ is of the form $\left(0,0,\left[X_{\mathcal{N}}, \mathcal{Q}_{0}\right]^{\perp}(\mathfrak{x})\right)$ where

$\left[X_{\mathcal{N}}, \mathcal{Q}_{0}\right]^{\perp}(\mathfrak{x})=\left(\left[i \Omega_{\perp}, \mathcal{Q}_{0}^{\perp}(\theta, y)\right]_{l i n}+(\omega+\varepsilon \widehat{\omega}) \cdot \partial_{\theta} \mathcal{Q}_{0}^{\perp}(\theta, y)+\nabla_{y} Q(y) \cdot \partial_{\theta} \mathcal{Q}_{0}^{\perp}(\theta, y)\right)[w]$.

One has

$$
\begin{gathered}
\omega \cdot \partial_{\theta} \mathcal{Q}_{0}^{\perp}(\theta, y)[w] \in \mathcal{O S}_{w}^{2}(N), \quad \varepsilon \widehat{\omega} \cdot \partial_{\theta} \mathcal{Q}_{0}^{\perp}(\theta, y)[w] \in \mathcal{O S}^{3}(N), \\
\nabla_{y} Q(y) \cdot \partial_{\theta} \mathcal{Q}_{0}^{\perp}(\theta, y)[w] \in \mathcal{O S}^{3}(N),
\end{gathered}
$$

and since $i \Omega_{\perp}$ is a Fourier multiplier of order three, it follows that $\left[i \Omega_{\perp}, \mathcal{Q}_{0}^{\perp}(\theta, y)\right]_{l i n} w$ $\in \mathcal{O S}_{w w}^{2}(N-3)$. The claimed statement then follows. 
(ii) Arguing as in the proof of Lemma 3.9(ii) (cf. (3.28)), and using that $F_{1}(\theta)[w, w]$ and $\mathcal{Q}_{1}^{\perp}(\theta)[w, w]$ are quadratic forms with respect to $w$, one sees that $Y:=$ $\left[X_{\mathcal{N}}, \mathcal{Q}_{1}\right]$ is of the form $Y=\left(Y^{(\theta)}, 0, Y^{\perp}\right)$ where

$$
\begin{aligned}
Y^{(\theta)}(\mathfrak{x})= & (\omega+\varepsilon \widehat{\omega}) \cdot \partial_{\theta} F_{1}(\theta)[w, w] \\
& -F_{1}(\theta)\left[\mathrm{i} \Omega_{\perp} w, w\right]-F_{1}(\theta)\left[w, \mathrm{i} \Omega_{\perp} w\right]+\nabla_{y} Q(y) \cdot \partial_{\theta} F_{1}(\theta)[w, w] \\
Y^{\perp}(\mathfrak{x})= & \mathrm{i} \Omega_{\perp} \mathcal{Q}_{1}^{\perp}(\theta)[w, w]-\mathcal{Q}_{1}^{\perp}(\theta)\left[\mathrm{i} \Omega_{\perp} w, w\right] \\
& -\mathcal{Q}_{1}^{\perp}(\theta)\left[w, \mathrm{i} \Omega_{\perp} w\right]+(\omega+\varepsilon \widehat{\omega}) \cdot \partial_{\theta} \mathcal{Q}_{1}^{\perp}(\theta)[w, w] \\
& +\nabla_{y} Q(y) \cdot \partial_{\theta} \mathcal{Q}_{1}^{\perp}(\theta)[w, w] .
\end{aligned}
$$

By (3.40), $\omega \cdot \partial_{\theta} F_{1}(\theta)[w, w], F_{1}(\theta)\left[i \Omega_{\perp} w, w\right]$, and $F_{1}(\theta)\left[w, \mathrm{i} \Omega_{\perp} w\right]$ are smooth functions and small of order two, whereas $\varepsilon \widehat{\omega} \cdot \partial_{\theta} F_{1}(\theta)[w, w]$ and $\nabla_{y} Q(y)$. $\partial_{\theta} F_{1}(\theta)[w, w]$ are smooth functions and small of order three. (Here we used that by (3.21), $\nabla_{y} Q(y)$ is small of order one.) Furthermore, by the definition of $\mathcal{Q}_{1}^{\perp}$ one has $\omega \cdot \partial_{\theta} \mathcal{Q}_{1}^{\perp}(\theta)[w, w] \in \mathcal{O S}_{w w}^{2}(N)$, whereas $\varepsilon \widehat{\omega} \cdot \partial_{\theta} \mathcal{Q}_{1}^{\perp}(\theta)[w, w]$ and $\nabla_{y} Q(y) \cdot \partial_{\theta} \mathcal{Q}_{1}^{\perp}(\theta)[w, w]$ are in $\mathcal{O S}^{3}(N)$. Finally, since $i \Omega_{\perp}$ is a Fourier multiplier of order three,

$\mathrm{i} \Omega_{\perp} \mathcal{Q}_{1}^{\perp}(\theta)[w, w]-\mathcal{Q}_{1}^{\perp}(\theta)\left[\mathrm{i} \Omega_{\perp} w, w\right]-\mathcal{Q}_{1}^{\perp}(\theta)\left[w, \mathrm{i} \Omega_{\perp} w\right] \in \mathcal{O S}_{w w}^{2}(N-3)$.

The claimed statement then follows.

(iii) By (1.36), $\Phi_{\mathcal{Q}}^{*} X_{\mathcal{N}}(\mathfrak{x})$ can be expanded as

$\Phi_{\mathcal{Q}}^{*} X_{\mathcal{N}}=X_{\mathcal{N}}+\left[X_{\mathcal{N}}, \mathcal{Q}\right]+Z, \quad Z(\mathfrak{x}):=\int_{0}^{1}(1-t) d \Phi_{\mathcal{Q}}(t, \mathfrak{x})^{-1}\left[\left[X_{\mathcal{N}}, \mathcal{Q}\right], \mathcal{Q}\right](\Phi(t, \mathfrak{x})) d t$

By items $(i)$ and $(i i)$, the commutator $\left[X_{\mathcal{N}}, \mathcal{Q}\right]$ is in $\mathcal{O S}^{2}(N-3)$, hence by Lemma 3.1, [[ $\left.\left.X_{\mathcal{N}}, \mathcal{Q}\right], \mathcal{Q}\right] \in \mathcal{O S}^{3}(N-3)$. By applying Lemma 3.17-(iii), one then infers that $Z \in \mathcal{O S}^{3}(N-3)$. The claimed expansion then follows by items (i) and $(i i)$.

In Sect. 5, we use Hamiltonian vector fields $X_{\mathcal{F}}$, corresponding to Hamiltonians $\mathcal{F}$, which are affine functions with respect to the normal component $w$. More precisely, $\mathcal{F}$ is assumed to be of the form

$$
\mathcal{F}(\mathfrak{x}):=\mathcal{F}_{0}(\theta, y)+\left\langle\mathcal{F}_{1}(\theta, y), w\right\rangle
$$

where

$$
\begin{aligned}
\mathcal{F}_{0} \in C_{b}^{\infty}\left(\mathbb{T}^{S_{+}} \times B_{S_{+}}(\delta) \times\left[0, \varepsilon_{0}\right], \mathbb{R}\right), & \\
& \mathcal{F}_{1} \in C_{b}^{\infty}\left(\mathbb{T}^{S_{+}} \times B_{S_{+}}(\delta) \times\left[0, \varepsilon_{0}\right], H_{\perp}^{S}\left(\mathbb{T}_{1}\right)\right), \quad \forall s \geq 0 .
\end{aligned}
$$

The Hamiltonian vector field generated by the Hamiltonian $\mathcal{F}$ is given by

$$
X_{\mathcal{F}}(\mathfrak{x})=\left(-\nabla_{\theta} \mathcal{F}(\mathfrak{x}), \nabla_{y} \mathcal{F}(\mathfrak{x}), \partial_{x} \mathcal{F}_{1}(\theta, y)\right) .
$$

The following lemma can be easily deduced by (3.41)-(3.43).

Lemma 3.19. The vector field $X_{\mathcal{F}}$ is a smoothing vector field of arbitrary order, i.e., $X_{\mathcal{F}} \in \mathcal{O S}(N)$ for any $N \in \mathbb{N}$. Moreover, if in addition $\mathcal{F}_{0}$ is small of order $p$ and $\mathcal{F}_{1}$ is small of order $q$, then $\nabla_{\theta} \mathcal{F}$ is small of order $\min \{p, q+1\}, \nabla_{y} \mathcal{F}$ is small of order $\min \{p-1, q\}$ and $\partial_{x} \mathcal{F}_{1}$ is small of order $q$. 


\section{Reformulation of Theorem 1.1 and Normal Form Theorem}

The goal of this section is to describe the normal form coordinates provided by [27, Theorem 1.1], specifically constructed to analyze perturbations of the $\mathrm{KdV}$ equations near finite gap solutions and then to express Eq. (1.4) with respect to these coordinates. The main results of this section are Theorem 4.2, which reformulates Theorem 1.1 in these novel coordinates, and Theorem 4.3 (Normal Form Theorem), which is the key ingredient into the proof of Theorem 4.2.

We begin by rephrasing [27, Theorem 1.1] in a form, adapted to our needs. Without further references, we use the notations introduced in Sect. 1.

Theorem 4.1. Let $S_{+} \subseteq \mathbb{N}$ be finite and $\Xi \subset \mathbb{R}_{>0}^{S_{+}}$be compact. Then for $\delta>0$ suffciently small with $\Xi+B_{S_{+}}(\delta) \subset \mathbb{R}_{>0}^{S_{+}}$there exists a $C^{\infty}$ - smooth family of canonical diffeomorphisms

$$
\Psi_{\mu}: \mathcal{V}(\delta) \rightarrow \Psi_{\mu}(\mathcal{V}(\delta)) \subseteq L_{0}^{2}\left(\mathbb{T}_{1}\right), \mathfrak{x} \mapsto q,
$$

parametrized by $\mu \in \Xi$, with the property that for any $\mu \in \Xi, \Psi_{\mu}(\mathfrak{x})$ satisfies

$$
\Psi_{\mu}(\theta, y, 0)=\Psi_{S_{+}}(\theta, \mu+y), \quad \forall(\theta, y, 0) \in \mathcal{V}(\delta),
$$

and is compatible with the scale of Sobolev spaces $H_{0}^{s}\left(\mathbb{T}_{1}\right), s \in \mathbb{Z}_{\geq 0}$ (meaning that $\Psi_{\mu}\left(\mathcal{V}(\delta) \cap \mathcal{E}_{s}\right) \subseteq H_{0}^{s}\left(\mathbb{T}_{1}\right)$ and $\Psi_{\mu}: \mathcal{V}(\delta) \cap \mathcal{E}_{s} \rightarrow H_{0}^{s}\left(\mathbb{T}_{1}\right)$ is a $C^{\infty}$-diffeomorphism onto its image), so that the following holds:

(AE1) For any $N \in \mathbb{N}, \mu \in \Xi$, and $\mathfrak{x}=(\theta, y, w) \in \mathcal{V}(\delta), \Psi(\mathfrak{x}) \equiv \Psi_{\mu}(\mathfrak{x})$ has an expansion of the form,

$$
\Psi(\mathfrak{x})=\Psi_{S_{+}}(\theta, \mu+y)+w+\sum_{k=1}^{N} a_{-k}(\mathfrak{x} ; \Psi) \partial_{x}^{-k} w+\mathcal{R}_{N}(\mathfrak{x} ; \Psi),
$$

where $\mathcal{R}_{N}(\theta, y, 0 ; \Psi)=0$ and where for any $s \in \mathbb{Z}_{\geq 0}$ and $1 \leq k \leq N$,

$$
\mathcal{V}(\delta) \rightarrow H^{s}\left(\mathbb{T}_{1}\right), \mathfrak{x} \mapsto a_{-k}(\mathfrak{x} ; \Psi), \quad \mathcal{V}^{s}(\delta) \rightarrow H^{s+N+1}\left(\mathbb{T}_{1}\right), \mathfrak{x} \mapsto \mathcal{R}_{N}(\mathfrak{x} ; \Psi),
$$

are $C^{\infty}$ maps (cf. (1.28) for the definition of $\left.\mathcal{V}^{s}(\delta)\right)$.

(AE2) For any $\mathfrak{x}=(\theta, y, w) \in \mathcal{V}^{1}(\delta)$ and $\mu \in \Xi$, the transpose $d \Psi_{\mu}(\mathfrak{x})^{\top}$ (with respect to the standard inner products) of the differential $d \Psi_{\mu}(\mathfrak{x}): E_{1} \rightarrow H_{0}^{1}\left(\mathbb{T}_{1}\right)$ yields a bounded operator $d \Psi(\mathfrak{x})^{\top} \equiv d \Psi_{\mu}(\mathfrak{x})^{\top}: H_{0}^{1}\left(\mathbb{T}_{1}\right) \rightarrow E_{1}$. For any $\widehat{q} \in H_{0}^{1}\left(\mathbb{T}_{1}\right)$ and any integer $N \geq 1, d \Psi(\mathfrak{x})^{\top}[\widehat{q}]$ admits an expansion of the form

$$
\begin{aligned}
d \Psi(\mathfrak{x})^{\top}[\widehat{q}]= & \left(0,0, \Pi_{\perp} \widehat{q}+\Pi_{\perp} \sum_{k=1}^{N} a_{-k}\left(\mathfrak{x} ; d \Psi^{\top}\right) \partial_{x}^{-k} \widehat{q}+\Pi_{\perp} \sum_{k=1}^{N}\left(\partial_{x}^{-k} w\right) \mathcal{A}_{-k}\left(\mathfrak{x} ; d \Psi^{\top}\right)[\widehat{q}]\right) \\
& +\mathcal{R}_{N}\left(\mathfrak{x} ; d \Psi^{\top}\right)[\widehat{q}]
\end{aligned}
$$

where for any $s \in \mathbb{N}$ and $1 \leq k \leq N$,

$\mathcal{V}^{1}(\delta) \rightarrow H^{s}\left(\mathbb{T}_{1}\right), \mathfrak{x} \mapsto a_{-k}\left(\mathfrak{x} ; d \Psi^{\top}\right), \quad \mathcal{V}^{1}(\delta) \rightarrow \mathcal{B}\left(H_{0}^{1}\left(\mathbb{T}_{1}\right), H^{s}\left(\mathbb{T}_{1}\right)\right), \mathfrak{x} \mapsto \mathcal{A}_{-k}\left(\mathfrak{x} ; d \Psi^{\top}\right)$,

and

$$
\mathcal{V}^{s}(\delta) \rightarrow \mathcal{B}\left(H_{0}^{s}\left(\mathbb{T}_{1}\right), E_{s+N+1}\right), \mathfrak{x} \mapsto \mathcal{R}_{N}\left(\mathfrak{x} ; d \Psi^{\top}\right),
$$

are $C^{\infty}$-smooth, bounded maps. 
(AE3) For any $\mu \in \Xi$, the Hamiltonian $\mathcal{H}_{\mu}^{k d v}:=H^{k d v} \circ \Psi_{\mu}: \mathcal{V}^{1}(\delta) \rightarrow \mathbb{R}$ is in normal form up to order three. More precisely, for any $\mathfrak{x}=(\theta, y, w) \in \mathcal{V}^{1}(\delta)$, the Taylor expansion of $\mathcal{H}^{k d v} \equiv \mathcal{H}_{\mu}^{k d v}$ at $(\theta, 0,0)$ with respect to $y$ and $w$ up to order three reads

$$
\mathcal{H}^{k d v}(\mathfrak{x})=e+\omega \cdot y+\frac{1}{2} \Omega_{S_{+}}[y] \cdot y+\frac{1}{2}\left\langle D_{\perp}^{-1} \Omega_{\perp} w, w\right\rangle+\mathcal{P}^{k d v}(\mathfrak{x}),
$$

where $e:=\mathcal{H}_{\mu}^{k d v}(0,0,0)=H^{k d v}\left(\Psi_{S_{+}}(0, \mu)\right)$,

$$
\omega=\left(\omega_{n}^{k d v}(\mu, 0)\right)_{n \in S_{+}}, \quad \Omega_{S_{+}}:=\left(\partial_{I_{j}} \omega_{k}^{k d v}(\mu, 0)\right)_{j, k \in S_{+}},
$$

and for any $w=\sum_{n \in S^{\perp}} w_{n} e^{\mathrm{i} 2 \pi n x}, D_{\perp}^{-1} w:=\sum_{n \in S^{\perp}} \frac{1}{2 \pi n} w_{n} e^{\mathrm{i} 2 \pi n x}$, and $(c f$. (1.18))

$$
\Omega_{\perp} w:=\sum_{n \in S^{\perp}} \Omega_{n} w_{n} e^{\mathrm{i} 2 \pi n x}, \quad \Omega_{n}:=\omega_{n}^{k d v}(\mu, 0), \quad \forall n \in S^{\perp} .
$$

Furthermore, $\mathcal{P}^{k d v}: \mathcal{V}^{1}(\delta) \rightarrow \mathbb{R}$ is $C^{\infty}$-smooth, satisfies

$$
\left|\mathcal{P}^{k d v}(\mathfrak{x})\right| \lesssim\left(|y|+\|w\|_{1}\right)^{3}, \quad \forall \mathfrak{x}=(\theta, y, w) \in \mathcal{V}^{1}(\delta), \forall \mu \in \Xi,
$$

and has the following property: for any integer $N \geq 1$ there exists an integer $\sigma_{N} \geq N$ (loss of regularity) so that $\nabla \mathcal{P}^{k d v}(\mathfrak{x})=\left(\nabla_{\theta} \mathcal{P}^{k d v}(\mathfrak{x}), \nabla_{y} \mathcal{P}^{k d v}(\mathfrak{x}), \nabla_{\perp}\right.$ $\left.\mathcal{P}^{k d v}(\mathfrak{x})\right)$ admits an expansion of the form

$$
\nabla \mathcal{P}^{k d v}(\mathfrak{x})=\left(0,0, \Pi_{\perp} \sum_{k=0}^{N} T_{a_{-k}\left(\mathfrak{x} ; \mathcal{P}^{k d v}\right)} \partial_{x}^{-k} w\right)+\mathcal{R}_{N}\left(\mathfrak{x} ; \mathcal{P}^{k d v}\right),
$$

where there exist integers $s_{N}>0$ and $\sigma_{N}>0$ so that for any $s \geq s_{N}$ and any $0 \leq k \leq N$,

$$
\mathcal{V}^{s+\sigma_{N}}(\delta) \rightarrow H^{s}\left(\mathbb{T}_{1}\right), \mathfrak{x} \mapsto a_{-k}\left(\mathfrak{x} ; \mathcal{P}^{k d v}\right), \quad \mathcal{V}^{s \vee \sigma_{N}}(\delta) \rightarrow \mathcal{E}_{s+N+1}, \mathfrak{x} \mapsto \mathcal{R}_{N}\left(\mathfrak{x} ; \mathcal{P}^{k d v}\right)
$$

are $C^{\infty}$-smooth and satisfy for any $\theta \in \mathbb{T}_{1}^{S_{+}}, \mu \in \Xi$,

$$
\begin{aligned}
& a_{-k}\left(\theta, 0,0 ; \mathcal{P}^{k d v}\right)=0, \quad \mathcal{R}_{N}\left(\theta, 0,0 ; \mathcal{P}^{k d v}\right)=0, \\
& \partial_{y} \mathcal{R}_{N}\left(\theta, 0,0 ; \mathcal{P}^{k d v}\right)=0, \quad d_{\perp} \mathcal{R}_{N}\left(\theta, 0,0 ; \mathcal{P}^{k d v}\right)=0 .
\end{aligned}
$$

Here $T_{a_{k}\left(\mathfrak{x} ; \mathcal{P}^{k d v}\right)}$ denotes the operator of para-multiplication with $a_{k}\left(\mathfrak{x} ; \mathcal{P}^{k d v}\right)(c f$. Definition 2.1).

Remark 4.1. Since $\Omega_{-n}=-\Omega_{n}$ for any $n \in S^{\perp}$ (cf. (1.12), (1.18)), the Fourier multiplyer $i \Omega_{\perp}$ is a real operator. In view of the expansion (4.1) and the identity $\partial_{x} D^{-1}=\mathrm{i}$, the component of the Hamiltonian vector field $\mathcal{H}_{\mu}^{k d v}$ in the normal direction is given by

$$
\partial_{x} \nabla_{\perp} \mathcal{H}^{k d v}(\mathfrak{x})=\mathrm{i} \Omega_{\perp} w+\partial_{x} \nabla_{\perp} \mathcal{P}^{k d v}(\mathfrak{x}) .
$$

Next, we want to express Eq. (1.4) in the normal form coordinates provided by Theorem 4.1. To this end we write the nonlinear vector field $F(u)$ in the coordinates $(\theta, y, z)$. Recall that $F(u)=\partial_{x} \nabla P_{f}(u)$ where $P_{f}(u):=\int_{0}^{1} f(x, u(x)) d x$ and $f$ is given by (1.7). 
Proposition 4.1. Let $N \in \mathbb{N}$. Then there exist integers $s_{N}>0, \sigma_{N}>0$ so that for any perturbation $P_{f}(u)=\int_{0}^{1} f(x, u(x)) d x$ with $f C^{\infty}$-smooth, the following holds. For any $\mu \in \Xi$, the gradient of

$$
\mathcal{P}_{f} \equiv \mathcal{P}_{f, \mu}:=P_{f} \circ \Psi_{\mu}: \mathcal{V}^{1}(\delta) \rightarrow \mathbb{R}
$$

admits an expansion of the form

$$
\nabla \mathcal{P}_{f}(\mathfrak{x})=\left(0,0, \Pi_{\perp} \sum_{k=0}^{N} T_{a_{-k}\left(\mathfrak{x} ; \nabla \mathcal{P}_{f}\right)} \partial_{x}^{-k} w\right)+\mathcal{R}_{N}\left(\mathfrak{x} ; \nabla \mathcal{P}_{f}\right),
$$

where for any $s \geq s_{N}$ and for any $0 \leq k \leq N$, the maps

$$
\mathcal{V}^{S+\sigma_{N}}(\delta) \rightarrow H^{S}\left(\mathbb{T}_{1}\right), \mathfrak{x} \mapsto a_{-k}\left(\mathfrak{x} ; \nabla \mathcal{P}_{f}\right), \quad \mathcal{V}^{s}(\delta) \rightarrow E_{s+N+1}, \mathfrak{x} \mapsto \mathcal{R}_{N}\left(\mathfrak{x} ; \nabla \mathcal{P}_{f}\right)
$$

are $C^{\infty}$-smooth.

Proof. One has

$$
\nabla P_{f}(u)(x)=\partial_{\zeta} f(x, u(x)) .
$$

By the Bony para-linearization formula (cf. [37, Section 5.2.3]) for the composition operator, one gets that

$$
\nabla P_{f}(u)(x)=\partial_{\zeta} f(x, u(x))=T_{\partial_{\zeta}^{2} f(x, u(x))} u+\mathcal{R}_{f}(u)
$$

where there exists $s_{N}>N$ (large) so that for any integer $s \geq s_{N}$, the map $\mathcal{R}_{f}$ : $H^{s}\left(\mathbb{T}_{1}\right) \rightarrow H^{s+N+1}\left(\mathbb{T}_{1}\right)$ is $C^{\infty}$-smooth. Note that $\mathcal{R}_{f}(u)$ contains the zeroth order term $\partial_{\zeta} f(x, 0)$ of the Taylor expansion of $\partial_{\zeta} f(x, \zeta)$ at $\zeta=0$. By Theorem 4.1-(AE2), $d \Psi(\mathfrak{x})^{\top}[\widehat{q}]$ has an expansion of the form

$$
\begin{aligned}
& \left(0,0, \Pi_{\perp}[\widehat{q}]+\Pi_{\perp} \sum_{k=1}^{N} a_{-k}\left(\mathfrak{x} ; d \Psi^{\top}\right) \partial_{x}^{-k} \widehat{q}+\Pi_{\perp} \sum_{k=1}^{N}\left(\partial_{x}^{-k} w\right) \mathcal{A}_{-k}\left(\mathfrak{x} ; d \Psi^{\top}\right)[\widehat{q}]\right) \\
& +\mathcal{R}_{N}\left(\mathfrak{x} ; d \Psi^{\top}\right)[\widehat{q}]
\end{aligned}
$$

where the maps $\mathcal{V}(\delta) \rightarrow H^{s}\left(\mathbb{T}_{1}\right), \mathfrak{x} \mapsto a_{k}\left(\mathfrak{x} ; d \Psi^{\top}\right)$,

$$
\begin{aligned}
& \mathcal{V}^{1}(\delta) \rightarrow \mathcal{B}\left(H_{0}^{1}\left(\mathbb{T}_{1}\right), H^{s}\left(\mathbb{T}_{1}\right)\right), \mathfrak{x} \mapsto \mathcal{A}_{k}\left(\mathfrak{x} ; d \Psi^{\top}\right), \\
& \mathcal{V}^{s}(\delta) \rightarrow \mathcal{B}\left(H_{0}^{s}\left(\mathbb{T}_{1}\right), E_{s+N+1}\right), \mathfrak{x} \mapsto \mathcal{R}_{N}\left(\mathfrak{x} ; d \Psi^{\top}\right),
\end{aligned}
$$

are $C^{\infty}$-smooth, bounded maps. Using the expansion of $\Psi(\mathfrak{x})$ provided by Theorem 4.1(AE1),

$$
\Psi(\mathfrak{x})=\Psi_{S_{+}}(\theta, \mu+y)+w+\sum_{k=1}^{N} a_{-k}(\mathfrak{x} ; \Psi) \partial_{x}^{-k} w+\mathcal{R}_{N}(\mathfrak{x} ; \Psi)
$$

together with the para-product formula (2.3) and Lemma 2.3, one obtains

$$
\begin{gathered}
\left(\nabla P_{f}\right)(\Psi(\mathfrak{x}))=\sum_{k=0}^{N} T_{a_{-k}\left(\mathfrak{x} ; \nabla P_{f} \circ \Psi\right)} \partial_{x}^{-k} w+\mathcal{R}_{N}\left(\mathfrak{x} ; \nabla P_{f} \circ \Psi\right), \\
a_{0}\left(\mathfrak{x} ; \nabla P_{f} \circ \Psi\right)=\partial_{\zeta}^{2} f(x, \Psi(\mathfrak{x})),
\end{gathered}
$$


where there exist integers $\sigma_{N} \geq 0$ and $s_{N} \geq 0$ so that for any $s \geq s_{N}$ and $0 \leq k \leq N$, the maps

$\mathcal{V}^{s+\sigma_{N}} \rightarrow H^{s}\left(\mathbb{T}_{1}\right), \mathfrak{x} \mapsto a_{-k}\left(\mathfrak{x} ; \nabla P_{f} \circ \Psi\right), \quad \mathcal{V}^{s}(\delta) \rightarrow E_{s+N+1}, \mathfrak{x} \mapsto \mathcal{R}_{N}\left(\mathfrak{x} ; \nabla P_{f} \circ \Psi\right)$,

are $C^{\infty}$-smooth. The expansion of $\nabla \mathcal{P}_{f}(\mathfrak{x})=d \Psi(\mathfrak{x})^{\top}\left(\nabla P_{f}\right)(\Psi(\mathfrak{x}))$ is then computed by using the one of $d \Psi(\mathfrak{x})^{\top}$, provided by Theorem 4.1-(AE2). For any $1 \leq k \leq N$, we thus need to compute the expansion of the sum

$$
\sum_{k=1}^{N} a_{-k}\left(\mathfrak{x} ; d \Psi^{\top}\right) \partial_{x}^{-k} \nabla P_{f}(\Psi(\mathfrak{x}))+\left(\partial_{x}^{-k} w\right) \mathcal{A}_{-k}\left(\mathfrak{x} ; d \Psi^{\top}\right)\left[\nabla P_{f}(\Psi(\mathfrak{x}))\right]
$$

By (4.8) and using the para-product formula (2.3) one obtains

$$
\begin{aligned}
\Pi_{\perp} & \sum_{k=1}^{N} a_{-k}\left(\mathfrak{x} ; d \Psi^{\top}\right) \partial_{x}^{-k} \nabla P_{f}(\Psi(\mathfrak{x}))+\left(\partial_{x}^{-k} w\right) \mathcal{A}_{-k}\left(\mathfrak{x} ; d \Psi^{\top}\right)\left[\nabla P_{f}(\Psi(\mathfrak{x}))\right] \\
= & \Pi_{\perp} \sum_{k=1}^{N}\left(T_{a_{-k}\left(\mathfrak{x} ; d \Psi^{\top}\right)} \partial_{x}^{-k} \nabla P_{f}(\Psi(\mathfrak{x}))+T_{\partial_{x}^{-k} \nabla P_{f}(\Psi(\mathfrak{x}))} a_{-k}\left(\mathfrak{x} ; d \Psi^{\top}\right)\right) \\
& +\mathcal{R}^{(B)}\left(a_{-k}\left(\mathfrak{x} ; d \Psi^{\top}\right), \partial_{x}^{-k} \nabla P_{f}(\Psi(\mathfrak{x}))\right) \\
& +\Pi_{\perp} \sum_{k=1}^{N} T_{\mathcal{A}_{-k}\left(\mathfrak{x} ; d \Psi^{\top}\right)\left[\nabla P_{f}(\Psi(\mathfrak{x}))\right]} \partial_{x}^{-k} w+T_{\partial_{x}^{-k} w^{-k}}\left(\mathfrak{x} ; d \Psi^{\top}\right)\left[\nabla P_{f}(\Psi(\mathfrak{x}))\right] \\
& +\Pi_{\perp} \sum_{k=1}^{N} \mathcal{R}^{(B)}\left(\mathcal{A}_{-k}\left(\mathfrak{x} ; d \Psi^{\top}\right)\left[\nabla P_{f}(\Psi(\mathfrak{x}))\right], \partial_{x}^{-k} w\right) \\
= & \Pi_{\perp} \sum_{k=1}^{N}\left(T_{a_{-k}\left(\mathfrak{x} ; d \Psi^{\top}\right)} \partial_{x}^{-k} \nabla P_{f}(\Psi(\mathfrak{x}))+T_{\mathcal{A}_{-k}\left(\mathfrak{x} ; d \Psi^{\top}\right)\left[\nabla P_{f}(\Psi(\mathfrak{x}))\right]} \partial_{x}^{-k} w\right)+\mathcal{R}_{N}^{(1)}(\mathfrak{x})
\end{aligned}
$$

where

$$
\begin{aligned}
\mathcal{R}_{N}^{(1)}(\mathfrak{x}):= & \Pi_{\perp} \sum_{k=1}^{N} T_{\partial_{x}^{-k} \nabla P_{f}(\Psi(\mathfrak{x}))} a_{-k}\left(\mathfrak{x} ; d \Psi^{\top}\right)+T_{\partial_{x}^{-k} w} \mathcal{A}_{-k}\left(\mathfrak{x} ; d \Psi^{\top}\right)\left[\nabla P_{f}(\Psi(\mathfrak{x}))\right] \\
& +\Pi_{\perp} \sum_{k=1}^{N}\left(\mathcal{R}^{(B)}\left(a_{-k}\left(\mathfrak{x} ; d \Psi^{\top}\right), \partial_{x}^{-k} \nabla P_{f}(\Psi(\mathfrak{x}))\right)\right. \\
& \left.+\mathcal{R}^{(B)}\left(\mathcal{A}_{-k}\left(\mathfrak{x} ; d \Psi^{\top}\right)\left[\nabla P_{f}(\Psi(\mathfrak{x}))\right], \partial_{x}^{-k} w\right)\right) .
\end{aligned}
$$

By applying Theorem 4.1-(AE1),(AE2), and Lemma 2.2, one obtains, after increasing $s_{N}$ if needed, that for any $s \geq s_{N}$, the map $\mathcal{V}^{s}(\delta) \rightarrow E_{s+N+1}, \mathfrak{x} \mapsto \mathcal{R}_{N}^{(1)}(\mathfrak{x})$ is $C^{\infty}$-smooth. By the expansion given in (4.8) and by applying Lemma 2.5 (composition of paradifferential operators), one then gets the following identity for the normal component 
$\left(\nabla \mathcal{P}_{f}\right)^{\perp}$ of $\nabla \mathcal{P}_{f}$,

$$
\begin{aligned}
\left(\nabla \mathcal{P}_{f}\right)^{\perp}(\mathfrak{x})= & \Pi_{\perp}\left[\nabla P_{f}(\Psi(\mathfrak{x}))\right]+\Pi_{\perp} \sum_{k=1}^{N}\left(T_{a_{-k}\left(\mathfrak{x} ; d \Psi^{\top}\right)} \partial_{x}^{-k} \nabla P_{f}(\Psi(\mathfrak{x}))\right. \\
& \left.+T_{\mathcal{A}_{-k}\left(\mathfrak{x} ; d \Psi^{\top}\right)\left[\nabla P_{f}(\Psi(\mathfrak{x}))\right]} \partial_{x}^{-k} w\right)+\mathcal{R}_{N}^{(1)}(\mathfrak{x}) \\
= & \Pi_{\perp} \sum_{k=0}^{N} T_{a_{-k}\left(\mathfrak{x} ; \nabla \mathcal{P}_{f}\right)} \partial_{x}^{-k} w+\mathcal{R}_{N}^{(2)}(\mathfrak{x}), \quad a_{0}\left(\mathfrak{x} ; \nabla \mathcal{P}_{f}\right)=\partial_{\zeta}^{2} f(x, w(x)),
\end{aligned}
$$

where there exist constants $s_{N} \geq N$ and $\sigma_{N} \geq N$ so that for any $s \geq s_{N}$ and any $0 \leq k \leq N$, the maps

$$
\mathcal{V}^{s+\sigma_{N}}(\delta) \rightarrow H^{s}\left(\mathbb{T}_{1}\right), \mathfrak{x} \mapsto a_{-k}\left(\mathfrak{x} ; \nabla \mathcal{P}_{f}\right), \quad \mathcal{V}^{s}(\delta) \rightarrow H_{\perp}^{s+N+1}\left(\mathbb{T}_{1}\right), \mathfrak{x} \mapsto \mathcal{R}_{N}^{(2)}(\mathfrak{x}),
$$

are $C^{\infty}$-smooth. Altogether we obtain

$$
\nabla \mathcal{P}_{f}(\mathfrak{x})=d \Psi(\mathfrak{x})^{\top}\left(\nabla P_{f}\right)(\Psi(\mathfrak{x}))=\left(0,0, \Pi_{\perp} \sum_{k=0}^{N} T_{a_{-k}\left(\mathfrak{x} ; \nabla \mathcal{P}_{f}\right)} \partial_{x}^{-k} w\right)+\mathcal{R}_{N}\left(\mathfrak{x} ; \nabla \mathcal{P}_{f}\right)
$$

where

$$
\mathcal{R}_{N}\left(\mathfrak{x} ; \nabla \mathcal{P}_{f}\right):=\left(0,0, \mathcal{R}_{N}^{(2)}(\mathfrak{x})\right)+\mathcal{R}_{N}\left(\mathfrak{x} ; d \Psi^{\top}\right)\left[\nabla P_{f}(\Psi(\mathfrak{x}))\right]
$$

One verifies in a straightforward way that $\mathcal{R}_{N}\left(\mathfrak{x} ; \nabla \mathcal{P}_{f}\right)$ has the stated properties.

Combining Theorem 4.1 and Proposition 4.1 together with Lemma 2.4 yields the following corollary.

Corollary 4.1 (Expansion of $\left.\mathcal{H}_{\mu}\right)$. For any $\mu \in \Xi, \mathcal{H} \equiv \mathcal{H}_{\mu}=\left(H^{k d v}+\varepsilon P_{f}\right) \circ \Phi_{\mu}$ can be written as

$$
\mathcal{H}(\mathfrak{x})=e+\mathcal{N}(\mathfrak{x})+\mathcal{P}(\mathfrak{x}), \quad \mathcal{P}(\mathfrak{x}):=\mathcal{P}^{k d v}(\mathfrak{x})+\varepsilon \mathcal{P}_{f}(\mathfrak{x})
$$

where $e, \mathcal{N}$, and $\mathcal{P}^{k d v}$ are given by Theorem 4.1-(AE3) and $\mathcal{P}_{f}$ by Proposition 4.1. More precisely, $e=\mathcal{H}_{\mu}^{k d v}(0,0,0)$ and for any $\mathfrak{x}=(\theta, y, w) \in \mathcal{V}^{1}(\delta)$,

$$
\mathcal{N}(y, w)=\omega \cdot y+\frac{1}{2} \Omega_{S_{+}}[y] \cdot y+\frac{1}{2}\left\langle D_{\perp}^{-1} \Omega_{\perp} w, w\right\rangle,
$$

with

$$
D_{\perp}^{-1} w(x)=\sum_{j \in S^{\perp}} \frac{1}{2 \pi n} w_{n} e^{\mathrm{i} 2 \pi n x}, \quad \Omega_{\perp} w(x)=\sum_{n \in S^{\perp}} \Omega_{n} w_{n} e^{\mathrm{i} 2 \pi n x}
$$

The perturbation $\mathcal{P}$ is of the form (cf. Proposition 4.1)

$$
\mathcal{P}(\mathfrak{x})=\varepsilon \mathcal{P}_{L}(\mathfrak{x})+\mathcal{P}_{e}(\mathfrak{x}), \quad \mathcal{P}_{L}(\mathfrak{x}):=\mathcal{P}_{00}(\theta)+\mathcal{P}_{10}(\theta) \cdot y+\left\langle\mathcal{P}_{01}(\theta), w\right\rangle
$$


with $\mathcal{P}_{e}, \mathcal{P}_{00}(\theta), \mathcal{P}_{10}(\theta)$, and $\mathcal{P}_{01}(\theta)$ having the following properties: there exist $0<$ $\delta<1,0<\varepsilon_{0}<1$, and an integer $\sigma>0$ so that

$\mathcal{P}_{00} \in C^{\infty}\left(\mathbb{T}^{S_{+}}, \mathbb{R}\right), \quad \mathcal{P}_{10} \in C^{\infty}\left(\mathbb{T}^{S_{+}}, \mathbb{R}^{S_{+}}\right), \quad \mathcal{P}_{01} \in C^{\infty}\left(\mathbb{T}^{S_{+}}, H_{\perp}^{S}\left(\mathbb{T}_{1}\right)\right), \quad \forall s \geq 0$, $\mathcal{P}_{e} \in C^{\infty}\left(\mathcal{V}^{\sigma}(\delta) \times\left[0, \varepsilon_{0}\right], \mathbb{R}\right)$ small of order three,

$X_{\mathcal{P}_{e}}=\left(X_{\mathcal{P}_{e}}^{(\theta)}, X_{\mathcal{P}_{e}}^{(y)}, X_{\mathcal{P}_{e}}^{\perp}\right)=\left(-\nabla_{y} \mathcal{P}_{e}, \nabla_{\theta} \mathcal{P}_{e}, \partial_{x} \nabla_{\perp} \mathcal{P}_{e}\right)$ small of order two,

$X_{\mathcal{P}_{e}}^{\perp}=\partial_{x} \nabla_{\perp} \mathcal{P}_{e}=\mathcal{O B}^{2}(1, N)+\mathcal{O S}^{2}(N), \quad \forall N \in \mathbb{N}$,

(cf. Definition 3.1 and Definition 3.3 for the classes of vector fields $\mathcal{O B}^{2}(1, N)$ and respectively, $\left.\mathcal{O S}^{2}(N)\right)$.

Remark 4.2. Since the constant $e$ in (4.10) does not affect the Hamiltonian vector field $X_{\mathcal{H}}$, by notational convenience, we will suppress it in the sequel. The same convention will be used for any Hamiltonian under consideration.

We now reformulate Theorem 1.1 in the coordinates, provided by Theorem 4.1. By Corollary 4.1, the one parameter family of Hamiltonians $\mathcal{H} \equiv \mathcal{H}_{\mu}=\left(H^{k d v}+\varepsilon P_{f}\right) \circ \Phi_{\mu}$, $\mu \in \Xi$, is given by

$$
\mathcal{H}(\mathfrak{x})=\mathcal{N}(\mathfrak{x})+\varepsilon \mathcal{P}_{L}(\mathfrak{x})+\mathcal{P}_{e}(\mathfrak{x})
$$

with $\mathcal{N}$ defined by (4.11) and $\mathcal{P}_{L}, \mathcal{P}_{e}$ by (4.13) (cf. Remark 4.2). Using that $\partial_{x} D_{\perp}^{-1} \Omega_{\perp}=$ $\mathrm{i} \Omega_{\perp}$, the Hamiltonian vector field $X_{\mathcal{H}}=\left(-\nabla_{y} \mathcal{H}, \nabla_{\theta} \mathcal{H}, \partial_{x} \nabla_{\perp} \mathcal{H}\right)$ can be computed as

$$
X_{\mathcal{H}}(\mathfrak{x})=\left(\begin{array}{c}
-\omega-\Omega_{S_{+}}[y]-\varepsilon \mathcal{P}_{10}(\theta)-\nabla_{y} \mathcal{P}_{e}(\mathfrak{x}) \\
\varepsilon \nabla_{\theta} \mathcal{P}_{L}(\mathfrak{x})+\nabla_{\theta} \mathcal{P}_{e}(\mathfrak{x}) \\
i \Omega_{\perp} w+\varepsilon \partial_{x} \mathcal{P}_{01}(\theta)+\partial_{x} \nabla_{\perp} \mathcal{P}_{e}(\mathfrak{x})
\end{array}\right)
$$

and the corresponding Hamiltonian equations are

$$
\begin{aligned}
\partial_{t} \theta & =-\omega-\Omega_{S_{+}} y-\varepsilon \mathcal{P}_{10}(\theta)-\nabla_{y} \mathcal{P}_{e}(\mathfrak{x}), \\
\partial_{t} y & =\varepsilon \nabla_{\theta} \mathcal{P}_{L}(\mathfrak{x})+\nabla_{\theta} \mathcal{P}_{e}(\mathfrak{x}), \\
\partial_{t} w & =\mathrm{i} \Omega_{\perp} w+\varepsilon \partial_{x} \mathcal{P}_{01}(\theta)+\partial_{x} \nabla_{\perp} \mathcal{P}_{e}(\mathfrak{x}) .
\end{aligned}
$$

Except for the measure estimate (1.23), Theorem 1.1 is an immediate consequence of the following theorem. (We refer to Sect. 8 for a proof of (1.23).)

Theorem 4.2. Let $f \in C^{\infty}\left(\mathbb{T}_{1} \times \mathbb{R}, \mathbb{R}\right), S_{+}$be a finite subset of $\mathbb{N}, \tau$ be a number with $\tau>\left|S_{+}\right|\left(c f\right.$. (1.20)), and $\mu=\mu(\omega)$ with $\omega \in \Pi_{\gamma}, 0<\gamma<1$. Then for any integer $s$ sufficiently large, there exists $0<\varepsilon_{0} \equiv \varepsilon_{0}(s, \gamma)<1$ with the following properties: for any $0<\varepsilon \leq \varepsilon_{0}$ there exists $T \equiv T_{\varepsilon, s, \gamma}=O\left(\varepsilon^{-2}\right)$, so that for any initial data $\mathfrak{x}_{0}=\left(\theta_{0}, y_{0}, w_{0}\right) \in \mathbb{T}^{S_{+}} \times \mathbb{R}^{S_{+}} \times H_{\perp}^{s}\left(\mathbb{T}_{1}\right)$, satisfying

$$
\left|y_{0}\right|,\left\|w_{0}\right\|_{s} \leq \varepsilon
$$

there exists a unique solution $t \mapsto \mathfrak{x}(t)=(\theta(t), y(t), w(t))$ of (4.17) with $\mathfrak{x}(0)=\mathfrak{x}_{0}$ and

$$
\begin{aligned}
\theta \in C^{1}\left([-T, T], \mathbb{T}^{S_{+}}\right), \quad y \in C^{1}\left([-T, T], \mathbb{R}^{S_{+}}\right), \\
\\
w \in C^{0}\left([-T, T], H_{\perp}^{s}\left(T_{1}\right)\right) \cap C^{1}\left([-T, T], H_{\perp}^{s-3}\left(\mathbb{T}_{1}\right)\right) .
\end{aligned}
$$

In addition, the solution satisfies $|y(t)|,\|w(t)\|_{s} \lesssim_{s, \gamma} \varepsilon$ for any $t \in[-T, T]$. 
Theorem 4.2 is proved in Sect. 7. A key ingredient of its proof is the following result on normal forms.

Theorem 4.3 (Normal Form Theorem). Let $f \in C^{\infty}\left(\mathbb{T}_{1} \times \mathbb{R}, \mathbb{R}\right), S_{+}$be a finite subset of $\mathbb{N}, \tau$ be a number with $\tau>\left|S_{+}\right|\left(c f\right.$. (1.20)), and $\mu=\mu(\omega)$ with $\omega \in \Pi_{\gamma}, 0<\gamma<1$. Then there exists $\sigma_{*}>0$ so that for any integer $s \geq \sigma_{*}$ the following holds: there exist $0<\delta \equiv \delta(s, \gamma)<1,0<\varepsilon_{0} \equiv \varepsilon_{0}(s, \gamma) \ll \delta$, and $C_{0} \equiv C_{0}(s, \gamma)>1$ with the property that for any $0<\varepsilon \leq \varepsilon_{0}$ there exists an invertible map $\Phi$ with inverse $\Phi^{-1}$ (cf. Remark 3.4),

$$
\Phi^{ \pm 1} \in \mathcal{C}_{b}^{\infty}\left(\mathcal{V}^{s}(\delta), \mathcal{V}^{s}\left(C_{0} \delta\right)\right), \quad \Phi^{ \pm 1}(\mathfrak{x})-\mathfrak{x} \text { small of order one }
$$

so that the pull back $X=\left(X^{(\theta)}, X^{(y)}, X^{\perp}\right):=\Phi^{*} X_{\mathcal{H}_{\mu}}$ of the vector field $X_{\mathcal{H}_{\mu}}$ by $\Phi$ has the form

$$
\begin{gathered}
X^{(\theta)}(\mathfrak{x})=-\omega-\varepsilon \widehat{\omega}+\mathrm{N}^{(\theta)}(y, w)+\mathcal{O}_{3}^{(\theta)}(\mathfrak{x}), \quad X^{(y)}(\mathfrak{x})=\mathcal{O}_{3}^{(y)}(\mathfrak{x}), \\
X^{\perp}(\mathfrak{x})=\mathrm{i} \Omega_{\perp} w+\mathrm{D}^{\perp}(\mathfrak{x})[w]+\Pi_{\perp} T_{a(\mathfrak{x})} \partial_{x} w+\mathcal{R}^{\perp}(\mathfrak{x}),
\end{gathered}
$$

where $\widehat{\omega} \in \mathbb{R}^{S_{+}}$and

$\mathrm{N}^{(\theta)} \in C_{b}^{\infty}\left(B_{S_{+}}(\delta) \times B_{\perp}^{\sigma_{*}}(\delta) \times\left[0, \varepsilon_{0}\right], \mathbb{R}^{S_{+}}\right)$small of order one (and independent of $\theta$ ),

$\mathcal{O}_{3}^{(\theta)}, \mathcal{O}_{3}^{(y)} \in C_{b}^{\infty}\left(\mathcal{V}^{\sigma_{*}}(\delta) \times\left[0, \varepsilon_{0}\right], \mathbb{R}^{S_{+}}\right)$small of order three,

$\mathrm{D}^{\perp} \in C_{b}^{\infty}\left(\mathcal{V}^{\sigma_{*}}(\delta) \times\left[0, \varepsilon_{0}\right], \mathcal{B}\left(H_{\perp}^{s}\left(\mathbb{T}_{1}\right), H_{\perp}^{s-1}\left(\mathbb{T}_{1}\right)\right)\right)$ small of order one,

$\mathrm{D}^{\perp}$ Fourier multiplier of the form $\mathrm{D}^{\perp}(\mathfrak{x})[w]=\sum_{j \in S^{\perp}} d_{j}(\mathfrak{x}) w_{j} e^{\mathrm{i} 2 \pi j x}$ with the properties

$d_{j} \in C_{b}^{\infty}\left(\mathcal{V}^{\sigma_{*}}(\delta) \times\left[0, \varepsilon_{0}\right], \mathbb{R}\right), \quad \forall j \in S^{\perp}, \quad \mathrm{D}^{\perp}$ skew-adjoint: $\mathrm{D}^{\perp}(\mathfrak{x})^{\top}=-\mathrm{D}^{\perp}(\mathfrak{x})$, $a \in C_{b}^{\infty}\left(\mathcal{V}^{s+\sigma_{*}}(\delta) \times\left[0, \varepsilon_{0}\right], H^{s}\left(\mathbb{T}_{1}\right)\right)$ small of order two,

$\mathcal{R}^{\perp} \in C_{b}^{\infty}\left(\mathcal{V}^{s}(\delta) \times\left[0, \varepsilon_{0}\right], H_{\perp}^{s}\left(\mathbb{T}_{1}\right)\right)$ small of order three.

The proof of Theorem 4.3 is given in Sect. 7. The transformation $\Phi$ is obtained as the composition of several transformations, constructed in Sects. 5-6.

\section{Smoothing Normal Form Steps}

As part of the proof of Theorem 4.3, the aim of this section is to normalize terms in the Taylor expansion of the Hamiltonian $\mathcal{H}$ (cf. (4.15)), which are affine with respect to the normal coordinate $w$ and homogeneous of order at most three with respect to the coordinates $y, w$ and the parameter $\varepsilon$ (cf. Overview of the proof of Theorem $1.1 \mathrm{in}$ Sect. 1). The main result of this section is the following one.

Proposition 5.1. Let $f \in C^{\infty}\left(\mathbb{T}_{1} \times \mathbb{R}, \mathbb{R}\right), S_{+}$be a finite subset of $\mathbb{N}$, $\tau$ be a number with $\tau>\left|S_{+}\right|\left(c f\right.$. (1.20)), and $\mu=\mu(\omega)$ with $\omega \in \Pi_{\gamma}, 0<\gamma<1$. Then for any $N \in \mathbb{N}$, there exist integers $s_{N}>0, \sigma_{N}>0$ so that for any $s \geq s_{N}$, there exist $0<\delta \equiv \delta(s, \gamma, N)<1$ and $0<\varepsilon_{0} \equiv \varepsilon_{0}(s, \gamma, N) \ll \delta$ with the following properties: for any $0<\varepsilon \leq \varepsilon_{0}$ there exists an invertible symplectic transformation $\Phi$ with inverse $\Phi^{-1}$ so that

$$
\Phi^{ \pm 1} \in \mathcal{C}_{b}^{\infty}\left(\mathcal{V}^{s}(\delta) \times\left[0, \varepsilon_{0}\right], \mathcal{V}^{s}(2 \delta)\right), \quad \Phi^{ \pm 1}(\mathfrak{x})-\mathfrak{x} \text { small of order one },
$$


and so that the Hamiltonian $\mathcal{H}^{(3)}:=\mathcal{H} \circ \Phi(c f$. (4.2)) has the form

$$
\mathcal{H}^{(3)}(\mathfrak{x})=\mathcal{N}^{(3)}(\mathfrak{x})+\mathcal{K}(\mathfrak{x}), \quad \mathcal{N}^{(3)}(\mathfrak{x}):=\omega \cdot y+\varepsilon \widehat{\omega} \cdot y+\frac{1}{2}\left\langle D_{\perp}^{-1} \Omega_{\perp} w, w\right\rangle+Q(y) .
$$

Here $\widehat{\omega} \equiv \widehat{\omega}(\varepsilon) \in \mathbb{R}^{S_{+}}$is an affine function of $\varepsilon, Q(y) \equiv Q(y, \varepsilon)$ is small of order two, a polynomial of degree three in $y$ and an affine function of $\varepsilon$, and the components of the Hamiltonian vector field $X_{\mathcal{K}}=\left(X_{\mathcal{K}}^{(\theta)}, X_{\mathcal{K}}^{(y)}, X_{\mathcal{K}}^{\perp}\right)=\left(-\nabla_{y} \mathcal{K}, \nabla_{\theta} \mathcal{K}, \partial_{x} \nabla_{\perp} \mathcal{K}\right)$, corresponding to the Hamiltonian $\mathcal{K}$, satisfy the following properties: $X_{\mathcal{K}}^{(\theta)}(\mathfrak{x})$ is of the form $\Upsilon_{2}^{(\theta)}(\theta)[w, w]+\Upsilon_{3}^{(\theta)}(\mathfrak{x})$ with

$$
\begin{aligned}
\Upsilon_{2}^{(\theta)} \in & C_{b}^{\infty}\left(\mathbb{T}^{S_{+}}, \mathcal{B}_{2}\left(H_{\perp}^{\sigma_{N}}\left(\mathbb{T}_{1}\right), \mathbb{R}^{S_{+}}\right)\right), \\
& \Upsilon_{3}^{(\theta)} \in C_{b}^{\infty}\left(\mathcal{V}^{\sigma_{N}}(\delta) \times\left[0, \varepsilon_{0}\right], \mathbb{R}^{S_{+}}\right), \text {small of order three, }
\end{aligned}
$$

and

$$
X_{\mathcal{K}}^{(y)} \in C_{b}^{\infty}\left(\mathcal{V}^{\sigma_{N}}(\delta) \times\left[0, \varepsilon_{0}\right], \mathbb{R}^{S_{+}}\right), \text {small of order three, } \quad X_{\mathcal{K}}^{\perp}(\mathfrak{x})=\Upsilon^{\perp}(\mathfrak{x})+\mathcal{R}_{N}^{\perp}(\mathfrak{x}),
$$

where

$$
\Upsilon^{\perp}=\mathcal{O B}_{w}^{2}(1, N)+\mathcal{O} \mathcal{B}_{w w}^{2}(1, N)+\mathcal{O B}^{3}(1, N), \quad \mathcal{R}_{N}^{\perp}=\mathcal{O S}_{w}^{2}(N)+\mathcal{O S}_{w w}^{2}(N)+\mathcal{O S}^{3}(N) .
$$

In the remaining part of this section we prove Proposition 5.1. The transformation $\Phi$ is obtained as the composition $\Phi^{(1)} \circ \Phi^{(2)} \circ \Phi^{(3)}$ of three symplectic transformations $\Phi^{(j)}, 1 \leq j \leq 3$.

\section{Normalization of $\mathcal{P}_{L}$ up to $O\left(\varepsilon^{2}\right)$.}

The aim of this first step is to construct a symplectic transformation $\Phi^{(1)}$ so that $\mathcal{P}_{L}(\mathfrak{x}) \stackrel{(4.13)}{=} \varepsilon\left(\mathcal{P}_{00}(\theta)+\mathcal{P}_{10}(\theta) \cdot y+\left\langle\mathcal{P}_{01}(\theta), w\right\rangle\right)$, when expressed in the new coordinates, is in normal form up to order $\varepsilon^{2}$. We construct $\Phi^{(1)}$ as the time one flow of a Hamiltonian flow corresponding to a Hamiltonian of the form

$$
\varepsilon \mathcal{F}^{(1)}(\mathfrak{x})=\varepsilon \mathcal{F}_{00}^{(1)}(\theta)+\varepsilon \mathcal{F}_{10}^{(1)}(\theta) \cdot y+\varepsilon\left\langle\mathcal{F}_{01}^{(1)}(\theta), w\right\rangle
$$

where

$$
\mathcal{F}_{00}^{(1)} \in C^{\infty}\left(\mathbb{T}^{S_{+}}, \mathbb{R}\right), \quad \mathcal{F}_{10}^{(1)} \in C^{\infty}\left(\mathbb{T}^{S_{+}}, \mathbb{R}^{S_{+}}\right), \quad \mathcal{F}_{01}^{(1)} \in C^{\infty}\left(\mathbb{T}^{S_{+}}, H_{\perp}^{n}\left(\mathbb{T}_{1}\right)\right), \quad \forall n \geq 0,
$$

will be chosen to serve our needs. The Hamiltonian vector field corresponding to the Hamiltonian $\varepsilon \mathcal{F}^{(1)}(\mathfrak{x})$,

$$
X_{\mathcal{E}} \mathcal{F}^{(1)}(\mathfrak{x})=\left(-\varepsilon \mathcal{F}_{10}^{(1)}(\theta), \varepsilon\left(\nabla_{\theta} \mathcal{F}_{00}^{(1)}(\theta)+\nabla_{\theta} \mathcal{F}_{10}^{(1)}(\theta) \cdot y+\nabla_{\theta}\left\langle\mathcal{F}_{01}^{(1)}(\theta), w\right\rangle\right), \varepsilon \partial_{x} \mathcal{F}_{01}^{(1)}(\theta)\right),
$$

is small of order one and by Lemma 3.19 arbitrarily smoothing. It means that $X_{\varepsilon} \mathcal{F}^{(1)} \in$ $\mathcal{O S}^{1}(N)$ for any integer $N \geq 1$ (cf. Definition 3.3). Denote by $\Phi^{(1)}(\tau, \cdot) \equiv \Phi_{\varepsilon} \mathcal{F}^{(1)}(\tau, \cdot)$ the flow of $X_{\varepsilon} \mathcal{F}^{(1)}$. For any given $N \in \mathbb{N}$, there exists an integer $s_{N}>0$ with the property that for any $s \geq s_{N}$, there exist $0<\delta \equiv \delta(s, \gamma, N)<1$ and $0<\varepsilon_{0} \equiv$ $\varepsilon_{0}(s, \gamma, N)<1$ (small), so that $\Phi^{(1)}(\tau, \cdot) \in C_{b}^{\infty}\left(\mathcal{V}^{s}(\delta) \times\left[0, \varepsilon_{0}\right], \mathcal{V}^{s}(2 \delta)\right)$ for any 
$-1 \leq \tau \leq 1$. The inverse of the time one flow map $\Phi^{(1)}:=\Phi^{(1)}(1, \cdot)$ is then given by $\left(\Phi^{(1)}\right)^{-1}=\Phi^{(1)}(-1, \cdot)$ (cf. Remark 3.4) and by Lemma 3.16,

$$
\Phi^{(1)}(\tau, \cdot)(\mathfrak{x})-\mathfrak{x} \in \mathcal{O S}^{1}(N), \quad \forall-1 \leq \tau \leq 1 .
$$

We now compute $\mathcal{H}^{(1)}:=\mathcal{H} \circ \Phi^{(1)}$ by separately expanding the terms appearing in (4.13). By (1.33) (Lie expansion), (5.5) (properties of $\Phi^{(1)}$ ) and (1.32) (Poisson bracket) one has

$$
\begin{aligned}
\mathcal{N} \circ \Phi^{(1)}= & \mathcal{N}+\varepsilon\left\{\mathcal{N}, \mathcal{F}^{(1)}\right\}+\varepsilon^{2} \int_{0}^{1}(1-\tau)\left\{\left\{\mathcal{N}, \mathcal{F}^{(1)}\right\}, \mathcal{F}^{(1)}\right\} \circ \Phi^{(1)}(\tau, \cdot) d \tau, \\
\left\{\mathcal{N}, \mathcal{F}^{(1)}\right\}= & \omega \cdot \partial_{\theta} \mathcal{F}_{00}^{(1)}(\theta)+\left(\omega \cdot \partial_{\theta} \mathcal{F}_{10}^{(1)}(\theta)+\Omega_{S_{+}}\left[\nabla_{\theta} \mathcal{F}_{00}^{(1)}(\theta)\right]\right) \cdot y \\
& +\left\langle\left(\omega \cdot \partial_{\theta}+\mathrm{i} \Omega_{\perp}\right) \mathcal{F}_{01}^{(1)}(\theta), w\right\rangle \\
& +\left(\Omega_{S_{+}}[y] \cdot \partial_{\theta}\right)\left(\mathcal{F}_{10}^{(1)}(\theta) \cdot y\right)+\left\langle\left(\Omega_{S_{+}}[y] \cdot \partial_{\theta}\right) \mathcal{F}_{01}^{(1)}(\theta), w\right\rangle
\end{aligned}
$$

and by (1.33) (Lie expansion) and (4.14) (properties of $\mathcal{P}_{e}$ )

$$
\begin{gathered}
\varepsilon \mathcal{P}_{L} \circ \Phi^{(1)}=\varepsilon \mathcal{P}_{L}+\varepsilon^{2} \int_{0}^{1}\left\{\mathcal{P}_{L}, \mathcal{F}^{(1)}\right\} \circ \Phi^{(1)}(\tau, \cdot) d \tau, \\
\mathcal{P}_{e} \circ \Phi^{(1)} C^{\infty}-\text { smooth, small of order three }
\end{gathered}
$$

Altogether, one obtains

$$
\begin{gathered}
\mathcal{H}^{(1)}=\mathcal{N}+\varepsilon\left(\omega \cdot \partial_{\theta} \mathcal{F}_{00}^{(1)}(\theta)+\mathcal{P}_{00}(\theta)\right)+\varepsilon\left(\omega \cdot \partial_{\theta} \mathcal{F}_{10}^{(1)}(\theta)+\mathcal{P}_{10}(\theta)+\Omega_{S_{+}}\left[\nabla_{\theta} \mathcal{F}_{00}^{(1)}(\theta)\right]\right) \cdot y \\
+\varepsilon\left\langle\left(\omega \cdot \partial_{\theta}+\mathrm{i} \Omega_{\perp}\right) \mathcal{F}_{01}^{(1)}+\mathcal{P}_{01}, w\right\rangle+\mathcal{P}^{(1)} \\
\mathcal{P}^{(1)}:=\varepsilon^{2} \int_{0}^{1}(1-\tau)\left\{\left\{\mathcal{N}, \mathcal{F}^{(1)}\right\}, \mathcal{F}^{(1)}\right\} \circ \Phi^{(1)}(\tau, \cdot) d \tau+\varepsilon^{2} \int_{0}^{1}\left\{\mathcal{P}_{L}, \mathcal{F}^{(1)}\right\} \circ \Phi^{(1)}(\tau, \cdot) d \tau \\
+\varepsilon\left(\Omega_{S_{+}}[y] \cdot \partial_{\theta}\right)\left(\mathcal{F}_{10}^{(1)}(\theta) \cdot y\right)+\varepsilon\left\langle\left(\Omega_{S_{+}}[y] \cdot \partial_{\theta}\right) \mathcal{F}_{01}^{(1)}(\theta), w\right\rangle+\mathcal{P}_{e} \circ \Phi^{(1)} .
\end{gathered}
$$

Since the terms appearing in the second line of (5.7) are small of order three, the Hamiltonian $\mathcal{P}^{(1)}$ admits an expansion of the form

$$
\mathcal{P}^{(1)}(\mathfrak{x})=\varepsilon^{2} \mathcal{P}_{00}^{(1)}(\theta)+\mathcal{P}_{e}^{(1)},
$$

where $\mathcal{P}_{00}^{(1)} \in C^{\infty}\left(\mathbb{T}^{S_{+}}, \mathbb{R}\right)$ and $\mathcal{P}_{e}^{(1)}$ is small of order three. In view of (5.6) and since $\Omega_{S_{+}}\left[\nabla_{\theta} \mathcal{F}_{00}^{(1)}\right]$ has zero average in $\theta$, we consider the following system of homological equations for $\mathcal{F}_{00}^{(1)}, \mathcal{F}_{10}^{(1)}, \mathcal{F}_{01}^{(1)}$,

$$
\left\{\begin{array}{l}
\omega \cdot \partial_{\theta} \mathcal{F}_{00}^{(1)}+\mathcal{P}_{00}=\left\langle\mathcal{P}_{00}\right\rangle_{\theta}, \\
\omega \cdot \partial_{\theta} \mathcal{F}_{10}^{(1)}+\mathcal{P}_{10}+\Omega_{S_{+}}\left[\nabla_{\theta} \mathcal{F}_{00}^{(1)}\right]=\left\langle\mathcal{P}_{10}\right\rangle_{\theta}, \\
\left(\omega \cdot \partial_{\theta}+\mathrm{i} \Omega_{\perp}\right) \mathcal{F}_{01}^{(1)}+\mathcal{P}_{01}=0
\end{array}\right.
$$

Since by assumption $\omega \in \Pi_{\gamma}, 0<\gamma<1$, (cf. (1.19)), we can apply Lemmata B.1, B.2, to conclude that the system (5.9) has a unique solution $\mathcal{F}_{00}^{(1)}, \mathcal{F}_{10}^{(1)}, \mathcal{F}_{01}^{(1)}$ satisfying (5.4) and $\left\langle\mathcal{F}_{00}^{(1)}\right\rangle_{\theta}=0,\left\langle\mathcal{F}_{10}^{(1)}\right\rangle_{\theta}=0$. The Hamiltonian $\mathcal{H}^{(1)}$, defined in (5.6), then reads

$$
\mathcal{H}^{(1)}=\mathcal{N}+\varepsilon \widehat{\mathcal{N}}_{1}+\varepsilon^{2} \mathcal{P}_{00}^{(1)}(\theta)+\mathcal{P}_{e}^{(1)}, \quad \widehat{\mathcal{N}}_{1}(y):=\left\langle\mathcal{P}_{00}\right\rangle_{\theta}+\left\langle\mathcal{P}_{10}\right\rangle_{\theta} \cdot y .
$$


Since $\mathcal{P}_{e}^{(1)}$ is small of order three, its Hamiltonian vector field $X_{\mathcal{P}_{e}^{(1)}}$ is small of order two. For later use we discuss the normal component $X_{\mathcal{P}_{e}^{(1)}}^{\perp}$ of the vector field $X_{\mathcal{P}_{e}^{(1)}}$. Since $X_{\varepsilon} \mathcal{F}^{(1)} \in \mathcal{O S}^{1}(N)$, and $X_{\mathcal{P}_{e}}^{\perp}=\mathcal{O B}^{2}(1, N)+\mathcal{O S}^{2}(N)$ (cf. (4.14)) it follows from Lemma 3.17 that $X_{\mathcal{P}_{e} \circ \Phi^{(1)}}^{\perp}=\mathcal{O B}^{2}(1, N)+\mathcal{O S}^{2}(N)$. Arguing similarly for all the other terms in the definition of $\mathcal{P}_{e}^{(1)}$ (cf. (5.7), (5.8)) one can show that

$$
X_{\mathcal{P}_{e}^{(1)}}^{\perp}=\partial_{x} \nabla_{\perp} \mathcal{P}_{e}^{(1)}=\mathcal{O B}^{2}(1, N)+\mathcal{O S}^{2}(N) .
$$

Normalization of $\varepsilon^{2} \mathcal{P}_{00}^{(1)}(\theta)$. The aim of this second step is to normalize the term $\varepsilon^{2} \mathcal{P}_{00}^{(1)}(\theta)$ (small of order 2) in (5.10). To this end we construct a symplectic transformation $\Phi^{(2)}$, given again by the time one flow of a Hamiltonian flow, corresponding to a Hamiltonian of the form $\varepsilon^{2} \mathcal{F}^{(2)}(\theta)$ with

$$
\mathcal{F}^{(2)} \in C^{\infty}\left(\mathbb{T}^{S_{+}}, \mathbb{R}\right)
$$

being a function to be determined. The Hamiltonian vector field corresponding to the Hamiltonian $\varepsilon^{2} \mathcal{F}^{(2)}(\theta)$,

$$
X_{\varepsilon^{2} \mathcal{F}^{(2)}}(\mathfrak{x})=\left(0, \varepsilon^{2} \nabla_{\theta} \mathcal{F}^{(2)}(\theta), 0\right) .
$$

is small of order two and by Lemma 3.19 arbitrarily smoothing. It means that $X_{\mathcal{E}^{2}} \mathcal{F}^{(2)} \in$ $\mathcal{O S}^{2}(N)$ for any integer $N \geq 1$ (cf. Definition 3.3). Denote by $\Phi^{(2)}(\tau, \cdot) \equiv \Phi_{\varepsilon^{2} \mathcal{F}^{(2)}}(\tau, \cdot)$

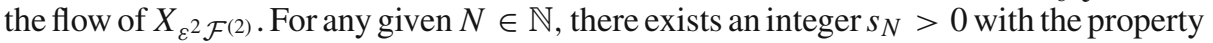
that for any $s \geq s_{N}$, there exist $0<\delta \equiv \delta(s, \gamma, N)<1$ and $0<\varepsilon_{0} \equiv \varepsilon_{0}(s, \gamma, N)<1$ (small), so that $\Phi^{(2)}(\tau, \cdot) \in C_{b}^{\infty}\left(\mathcal{V}^{s}(\delta) \times\left[0, \varepsilon_{0}\right], \mathcal{V}^{s}(2 \delta)\right)$ for any $-1 \leq \tau \leq 1$. The inverse of the time one flow map $\Phi^{(2)}:=\Phi^{(2)}(1, \cdot)$ is then given by $\left(\Phi^{(2)}\right)^{-1}=$ $\Phi^{(2)}(-1, \cdot)$ (cf. Remark 3.4) and by Lemma 3.16,

$$
\Phi^{(2)}(\tau, \cdot)(\mathfrak{x})-\mathfrak{x} \in \mathcal{O S}^{2}(N), \quad \forall-1 \leq \tau \leq 1 .
$$

We now compute $\mathcal{H}^{(2)}:=\mathcal{H}^{(1)} \circ \Phi^{(2)}$ by separately expanding the terms in (5.10). By (1.33) (Lie expansion), (5.13) (properties of $\Phi^{(2)}$ ) and (1.32) (Poisson bracket) one has

$$
\begin{aligned}
& \mathcal{N} \circ \Phi^{(2)}=\mathcal{N}+\varepsilon^{2}\left\{\mathcal{N}, \mathcal{F}^{(2)}\right\}+\varepsilon^{4} \int_{0}^{1}(1-\tau)\left\{\left\{\mathcal{N}, \mathcal{F}^{(2)}\right\}, \mathcal{F}^{(2)}\right\} \circ \Phi^{(2)}(\tau, \cdot) d \tau \\
& =\mathcal{N}+\varepsilon^{2} \omega \cdot \partial_{\theta} \mathcal{F}^{(2)}(\theta)+\varepsilon^{4} \int_{0}^{1}(1-\tau)\left\{\left\{\mathcal{N}, \mathcal{F}^{(2)}\right\}, \mathcal{F}^{(2)}\right\} \circ \Phi^{(2)}(\tau, \cdot) d \tau, \\
& \varepsilon \widehat{\mathcal{N}}_{1} \circ \Phi^{(2)}=\varepsilon \widehat{\mathcal{N}}_{1}+\varepsilon^{3} \int_{0}^{1}\left\{\widehat{\mathcal{N}}_{1}, \mathcal{F}^{(2)}\right\} \circ \Phi^{(2)}(\tau, \cdot) d \tau, \\
& \varepsilon^{2} \mathcal{P}_{00}^{(1)} \circ \Phi^{(2)}=\varepsilon^{2} \mathcal{P}_{00}^{(1)}(\theta)+\varepsilon^{4} \int_{0}^{1}\left\{\mathcal{P}_{00}^{(1)}, \mathcal{F}^{(2)}\right\} \circ \Phi^{(2)}(\tau, \cdot) d \tau \\
& \mathcal{P}_{e}^{(1)} \circ \Phi^{(2)} C^{\infty}-\text { smooth, small of order three. }
\end{aligned}
$$

Altogether, one obtains

$$
\mathcal{H}^{(2)}=\mathcal{H}^{(1)} \circ \Phi^{(2)}=\mathcal{N}+\varepsilon \widehat{\mathcal{N}}_{1}+\varepsilon^{2}\left(\omega \cdot \partial_{\theta} \mathcal{F}^{(2)}(\theta)+\mathcal{P}_{00}^{(1)}(\theta)\right)+\mathcal{P}^{(2)},
$$




$$
\begin{aligned}
\mathcal{P}^{(2)}:= & \varepsilon^{4} \int_{0}^{1}(1-\tau)\left\{\left\{\mathcal{N}, \mathcal{F}^{(2)}\right\}, \mathcal{F}^{(2)}\right\} \circ \Phi^{(2)}(\tau, \cdot) d \tau+\varepsilon^{3} \int_{0}^{1}\left\{\widehat{\mathcal{N}}_{1}, \mathcal{F}^{(2)}\right\} \circ \Phi^{(2)}(\tau, \cdot) d \tau \\
& +\varepsilon^{4} \int_{0}^{1}\left\{\mathcal{P}_{00}^{(1)}, \mathcal{F}^{(2)}\right\} \circ \Phi^{(2)}(\tau, \cdot) d \tau+\mathcal{P}_{e}^{(1)} \circ \Phi^{(2)}
\end{aligned}
$$

Since $\mathcal{P}_{e}^{(1)}$ is $C^{\infty}$-smooth and small of order three, so is $\mathcal{P}^{(2)}$. In view of the formula for $\mathcal{H}^{(2)}$ in (5.14) we consider the following homological equation for $\mathcal{F}^{(2)}$,

$$
\omega \cdot \partial_{\theta} \mathcal{F}^{(2)}(\theta)+\mathcal{P}_{00}^{(1)}(\theta)=\left\langle\mathcal{P}_{00}^{(1)}\right\rangle_{\theta} .
$$

Since by assumption $\omega \in \Pi_{\gamma}, 0<\gamma<1$, (cf. (1.19)), we can apply Lemmata B.1, B.2, to conclude that $(5.15)$ has a unique solution $\mathcal{F}^{(2)} \in C^{\infty}\left(\mathbb{T}^{S_{+}}, \mathbb{R}\right)$ with $\left\langle\mathcal{F}^{(2)}\right\rangle_{\theta}=0$. The Hamiltonian $\mathcal{H}^{(2)}$ in $(5.14)$ then reads

$$
\mathcal{H}^{(2)}=\mathcal{N}+\varepsilon \widehat{\mathcal{N}}_{2}+\mathcal{P}^{(2)}, \quad \widehat{\mathcal{N}}_{2}:=\widehat{\mathcal{N}}_{1}+\varepsilon\left\langle\mathcal{P}_{00}^{(1)}\right\rangle_{\theta} \stackrel{(5.10)}{=}\left\langle\mathcal{P}_{00}\right\rangle_{\theta}+\left\langle\mathcal{P}_{10}\right\rangle_{\theta} \cdot y+\varepsilon\left\langle\mathcal{P}_{00}^{(1)}\right\rangle_{\theta} .
$$

Since $\mathcal{P}^{(2)}$ is small of order three, its Hamiltonian vector field $X_{\mathcal{P}^{(2)}}$ is small of order two. For later use, we again discuss the normal component $X_{\mathcal{P}^{(2)}}^{\perp}$ of the vector field $X_{\mathcal{P}^{(2)}}$. Since $X_{\varepsilon^{2} \mathcal{F}^{(2)}} \in \mathcal{O S}^{2}(N)$, and $X_{\mathcal{P}_{e}^{(1)}}^{\perp}=\mathcal{O B}^{2}(1, N)+\mathcal{O S}^{2}(N)$ (cf. (5.11)) it follows from Lemma 3.17 that $X_{\mathcal{P}_{e}^{(1)} \circ \Phi^{(2)}}^{\perp}=\mathcal{O B}^{2}(1, N)+\mathcal{O S}^{2}(N)$. Arguing similarly for all the other terms in $\mathcal{P}^{(2)}$ (cf. (5.14) (5.7), (5.8), (5.10)) one shows that

$$
X_{\mathcal{P}^{(2)}}^{\perp}=\partial_{x} \nabla_{\perp} \mathcal{P}^{(2)}=\mathcal{O B}^{2}(1, N)+\mathcal{O S}^{2}(N) .
$$

Normalization of terms affine in $w$. The aim of this third step is to construct a symplectic coordinate transformation $\Phi^{(3)}$, normalizing the terms in the Taylor expansion of $\mathcal{P}^{(2)}$ (cf. (5.16)) with respect to $y, w$ at $(y, w)=(0,0)$, which are homogeneous in $y, w, \varepsilon$ of order three, of degree at most one in $w$, and of degree at most two in $\varepsilon$. The Taylor expansion of $\mathcal{P}^{(2)}$ in $y, w, \varepsilon$ up to order four reads

$$
\begin{aligned}
\mathcal{P}^{(2)}(\mathfrak{x})=\varepsilon^{3} & \mathcal{P}_{00}^{(2)}(\theta)+\varepsilon^{2}\left(\mathcal{P}_{10}^{(2)}(\theta) \cdot y+\left\langle\mathcal{P}_{01}^{(2)}(\theta), w\right\rangle\right) \\
& +\varepsilon\left(\mathcal{P}_{20}^{(2)}(\theta)[y, y]+\left\langle\mathcal{P}_{11}^{(2)}(\theta)[y], w\right\rangle\right)+\left\langle\mathcal{P}_{02}^{(2)}(\theta, y)[w], w\right\rangle \\
& +\mathcal{P}_{30}^{(2)}(\theta)[y, y, y]+\left\langle\mathcal{P}_{21}^{(2)}(\theta)[y, y], w\right\rangle+\mathcal{P}_{03}^{(2)}(\theta)[w, w, w]+\mathcal{O}_{4}(\mathfrak{x}),
\end{aligned}
$$

where for any $n \geq 0$,

$$
\begin{aligned}
& \mathcal{P}_{00}^{(2)} \in C^{\infty}\left(\mathbb{T}^{S_{+}}, \mathbb{R}\right), \quad \mathcal{P}_{10}^{(2)} \in C^{\infty}\left(\mathbb{T}^{S_{+}}, \mathbb{R}^{S_{+}}\right), \quad \mathcal{P}_{01}^{(2)} \in C^{\infty}\left(\mathbb{T}^{S_{+}}, H_{\perp}^{n}\left(\mathbb{T}_{1}\right)\right), \\
& \mathcal{P}_{20}^{(2)} \in C^{\infty}\left(\mathbb{T}^{S_{+}}, \mathcal{B}_{2}\left(\mathbb{R}^{S_{+}}\right)\right), \quad \mathcal{P}_{11}^{(2)} \in C^{\infty}\left(\mathbb{T}^{S_{+}}, \mathcal{B}\left(\mathbb{R}^{S_{+}}, H_{\perp}^{n}\left(\mathbb{T}_{1}\right)\right)\right), \\
& \mathcal{P}_{30}^{(2)} \in C^{\infty}\left(\mathbb{T}^{S_{+}}, \mathcal{B}_{3}\left(\mathbb{R}^{S_{+}}\right)\right), \quad \mathcal{P}_{21}^{(2)} \in C^{\infty}\left(\mathbb{T}^{S_{+}}, \mathcal{B}_{2}\left(\mathbb{R}^{S_{+}}, H_{\perp}^{n}\left(\mathbb{T}_{1}\right)\right)\right), \\
& \mathcal{P}_{03}^{(2)} \in C^{\infty}\left(\mathbb{T}^{S_{+}}, \mathcal{B}_{3}\left(H_{\perp}^{n}\left(\mathbb{T}_{1}\right)\right), \quad \mathcal{P}_{02}^{(2)} \in C^{\infty}\left(\mathbb{T}^{S_{+}} \times \mathbb{R}^{S_{+}} \times \mathbb{R}, \mathcal{B}\left(H_{\perp}^{n}\left(\mathbb{T}_{1}\right)\right)\right),\right. \\
& \mathcal{O}_{4}(\mathfrak{x}) C^{\infty} \text {-smooth, small of order four. }
\end{aligned}
$$

Remark 5.1. In the above Taylor expansion of $\mathcal{P}^{(2)}$, we combined the terms which are of the order ( 02 ) and (12) in the variables $y, w$ and for notational convenience, denoted the combined term by $\left\langle\mathcal{P}_{02}^{(2)}(\theta, y)[w], w\right\rangle$. The map

$$
\mathcal{P}_{02}^{(2)}:(\theta, y, \varepsilon) \mapsto \mathcal{P}_{02}^{(2)}(\theta, y) \equiv \mathcal{P}_{02}^{(2)}(\theta, y, \varepsilon)
$$

is linear in $y, \varepsilon$. 
We split $\mathcal{P}^{(2)}$ as $\mathcal{P}^{(2)}=\mathcal{P}_{1}^{(2)}+\mathcal{P}_{2}^{(2)}+\mathcal{O}_{4}$ where

$$
\begin{aligned}
\mathcal{P}_{1}^{(2)}:= & \varepsilon^{2} \mathcal{P}_{10}^{(2)}(\theta) \cdot y+\varepsilon \mathcal{P}_{20}^{(2)}(\theta)[y, y]+\mathcal{P}_{30}^{(2)}(\theta)[y, y, y] \\
& +\varepsilon^{2}\left\langle\mathcal{P}_{01}^{(2)}(\theta), w\right\rangle+\varepsilon\left\langle\mathcal{P}_{11}^{(2)}(\theta)[y], w\right\rangle+\left\langle\mathcal{P}_{21}^{(2)}(\theta)[y, y], w\right\rangle \\
\mathcal{P}_{2}^{(2)}:= & \varepsilon^{3} \mathcal{P}_{00}^{(2)}(\theta)+\left\langle\mathcal{P}_{02}^{(2)}(\theta, y)[w], w\right\rangle+\mathcal{P}_{03}^{(2)}(\theta)[w, w, w] .
\end{aligned}
$$

Note that $\mathcal{P}_{1}^{(2)}$ is affine in $w$ and that the Hamiltonian vector field corresponding to the term $\varepsilon^{3} \mathcal{P}_{00}^{(2)}(\theta)$ is small of order three. The transformation $\Phi^{(3)}$ is then defined as the time one flow of the Hamiltonian vector field $X_{\mathcal{F}^{(3)}}$ with a Hamiltonian $\mathcal{F}^{(3)}$ of the form

$$
\begin{array}{r}
\mathcal{F}^{(3)}(\mathfrak{x}):=\varepsilon^{2} \mathcal{F}_{10}^{(3)}(\theta) \cdot y+\varepsilon \mathcal{F}_{20}^{(3)}(\theta)[y, y]+\mathcal{F}_{30}^{(3)}(\theta)[y, y, y] \\
+\varepsilon^{2}\left\langle\mathcal{F}_{01}^{(3)}(\theta), w\right\rangle+\varepsilon\left\langle\mathcal{F}_{11}^{(3)}(\theta)[y], w\right\rangle+\left\langle\mathcal{F}_{21}^{(3)}(\theta)[y, y], w\right\rangle
\end{array}
$$

satisfying for any $n \geq 0$,

$$
\begin{aligned}
& \mathcal{F}_{10}^{(3)} \in C^{\infty}\left(\mathbb{T}^{S_{+}}, \mathbb{R}^{S_{+}}\right), \quad \mathcal{F}_{20}^{(3)} \in C^{\infty}\left(\mathbb{T}^{S_{+}}, \mathcal{B}_{2}\left(\mathbb{R}^{S_{+}}\right)\right), \\
& \mathcal{F}_{30}^{(3)} \in C^{\infty}\left(\mathbb{T}^{S_{+}}, \mathcal{B}_{3}\left(\mathbb{R}^{S_{+}}\right)\right), \quad \mathcal{F}_{01}^{(3)} \in C^{\infty}\left(\mathbb{T}^{S_{+}}, H_{\perp}^{n}\left(\mathbb{T}_{1}\right)\right), \\
& \mathcal{F}_{11}^{(3)} \in C^{\infty}\left(\mathbb{T}^{S_{+}}, \mathcal{B}\left(\mathbb{R}^{S_{+}}, H_{\perp}^{n}\left(\mathbb{T}_{1}\right)\right)\right), \quad \mathcal{F}_{21}^{(3)} \in C^{\infty}\left(\mathbb{T}^{S_{+}}, \mathcal{B}_{2}\left(\mathbb{R}^{S_{+}}, H_{\perp}^{n}\left(\mathbb{T}_{1}\right)\right)\right) .
\end{aligned}
$$

The functions $\mathcal{F}_{i j}^{(3)}$ will be chosen according to our needs. By (5.20), (5.21), the Hamiltonian vector field $X_{\mathcal{F}^{(3)}}$ is small of order two and by Lemma 3.19 arbitrarily smoothing. It means that $X_{\mathcal{F}^{(3)}} \in \mathcal{O S}^{2}(N)$ for any integer $N \geq 1$ (cf. Definition 3.3). Denote by $\Phi^{(3)}(\tau, \cdot) \equiv \Phi_{\mathcal{F}^{(3)}}(\tau, \cdot)$ the flow of $X_{\mathcal{F}^{(3)}}$. For any given $N \in \mathbb{N}$, there exists an integer $s_{N}>0$ with the property that for any $s \geq s_{N}$, there exist $0<\delta \equiv \delta(s, \gamma, N)<1$ and $0<\varepsilon_{0} \equiv \varepsilon_{0}(s, \gamma, N)<1$ (small), so that $\Phi^{(3)}(\tau, \cdot) \in C_{b}^{\infty}\left(\mathcal{V}^{s}(\delta) \times\left[0, \varepsilon_{0}\right], \mathcal{V}^{s}(2 \delta)\right.$ ) for any $-1 \leq \tau \leq 1$. The inverse of the time one flow map $\Phi^{(3)}:=\Phi^{(3)}(1, \cdot)$ is then given by $\left(\Phi^{(3)}\right)^{-1}=\Phi^{(3)}(-1, \cdot)$ and by Lemma 3.16,

$$
\Phi^{(3)}(\tau, \cdot)(\mathfrak{x})-\mathfrak{x} \in \mathcal{O S}^{2}(N), \quad \forall-1 \leq \tau \leq 1 .
$$

We now compute $\mathcal{H}^{(3)}:=\mathcal{H}^{(2)} \circ \Phi^{(3)}$ by expanding separately the terms in (5.16). By (1.33) (Lie expansion), (5.22) (properties of $\left.\Phi^{(3)}\right)$, (5.19) (splitting of $\left.\mathcal{P}^{(2)}\right),(5.20)-$ (5.21) (properties of $\mathcal{F}^{(3)}$, and (1.32) (Poisson bracket)

$$
\mathcal{N} \circ \Phi^{(3)}=\mathcal{N}+\left\{\mathcal{N}, \mathcal{F}^{(3)}\right\}+\int_{0}^{1}(1-\tau)\left\{\left\{\mathcal{N}, \mathcal{F}^{(3)}\right\}, \mathcal{F}^{(3)}\right\} \circ \Phi^{(3)}(\tau, \cdot) d \tau
$$

can be expanded as

$$
\begin{aligned}
\mathcal{N} \circ \Phi^{(3)}= & \mathcal{N}+\varepsilon^{2}\left(\omega \cdot \partial_{\theta}\right) \mathcal{F}_{10}^{(3)}(\theta) \cdot y+\varepsilon\left(\omega \cdot \partial_{\theta}\right) \mathcal{F}_{20}^{(3)}(\theta)[y, y]+\left(\omega \cdot \partial_{\theta}\right) \mathcal{F}_{30}^{(3)}(\theta)[y, y, y] \\
& +\varepsilon^{2}\left\{\left(\omega \cdot \partial_{\theta}+i \Omega_{\perp}\right) \mathcal{F}_{01}^{(3)}(\theta), w\right\rangle+\varepsilon\left\{\left(\omega \cdot \partial_{\theta}+i \Omega_{\perp}\right) \mathcal{F}_{11}^{(3)}(\theta)[y], w\right\rangle \\
& +\left\langle\left(\omega \cdot \partial_{\theta}+i \Omega_{\perp}\right) \mathcal{F}_{21}^{(3)}(\theta)[y, y], w\right\rangle \\
& +\left(\Omega_{S_{+}}[y] \cdot \partial_{\theta}\right) \mathcal{F}^{(3)}+\int_{0}^{1}(1-\tau)\left\{\left\{\mathcal{N}, \mathcal{F}^{(3)}\right\}, \mathcal{F}^{(3)}\right\} \circ \Phi^{(3)}(\tau, \cdot) d \tau \\
\widehat{\mathcal{N}}_{2} \circ \Phi^{(3)}= & \widehat{\mathcal{N}}_{2}+\int_{0}^{1}\left\{\widehat{\mathcal{N}}_{2}, \mathcal{F}^{(3)}\right\} \circ \Phi^{(3)}(\tau, \cdot) d \tau,
\end{aligned}
$$




$$
\mathcal{P}^{(2)} \circ \Phi^{(3)}=\mathcal{P}_{1}^{(2)}+\mathcal{P}_{2}^{(2)}+\int_{0}^{1}\left\{\mathcal{P}^{(2)}, \mathcal{F}^{(3)}\right\} \circ \Phi^{(3)}(\tau, \cdot) d \tau .
$$

Since $\mathcal{P}^{(2)}$ (cf. (5.16)), $\mathcal{F}^{(3)}$ (cf. (5.20)) are small of order three and in view of the definition of $\mathcal{N}, \widehat{\mathcal{N}}_{2}$ (cf. (5.16)), $\left\{\left\{\mathcal{N}, \mathcal{F}^{(3)}\right\}, \mathcal{F}^{(3)}\right\}, \varepsilon\left\{\widehat{\mathcal{N}}_{2}, \mathcal{F}^{(3)}\right\}$, and $\left\{\mathcal{P}^{(2)}, \mathcal{F}^{(3)}\right\}$ are small of order four. Hence the Hamiltonian $\mathcal{H}^{(3)}$ takes the form

$$
\begin{aligned}
\mathcal{H}^{(3)}= & \mathcal{N}+\varepsilon \widehat{\mathcal{N}}_{2}+\varepsilon^{2}\left(\omega \cdot \partial_{\theta} \mathcal{F}_{10}^{(3)}(\theta)+\mathcal{P}_{10}^{(2)}(\theta)\right) \cdot y+\varepsilon\left(\omega \cdot \partial_{\theta} \mathcal{F}_{20}^{(3)}(\theta)+\mathcal{P}_{20}^{(2)}(\theta)\right)[y, y] \\
+ & \left(\omega \cdot \partial_{\theta} \mathcal{F}_{30}^{(3)}(\theta)+\mathcal{P}_{30}^{(2)}(\theta)\right)[y, y, y]+\varepsilon^{2}\left\langle\left(\omega \cdot \partial_{\theta}+i \Omega_{\perp}\right) \mathcal{F}_{01}^{(3)}(\theta)+\mathcal{P}_{01}^{(2)}(\theta), w\right\rangle \\
+ & \varepsilon\left\langle\left(\omega \cdot \partial_{\theta}+i \Omega_{\perp}\right) \mathcal{F}_{11}^{(3)}(\theta)[y]+\mathcal{P}_{11}^{(2)}(\theta)[y], w\right\rangle \\
& +\left\langle\left(\omega \cdot \partial_{\theta}+i \Omega_{\perp}\right) \mathcal{F}_{21}^{(3)}(\theta)[y, y]+\mathcal{P}_{21}^{(2)}(\theta)[y, y], w\right\rangle+\mathcal{P}_{2}^{(2)}+\mathcal{O}_{4}
\end{aligned}
$$

where $\mathcal{O}_{4}$ comprises all the terms which are small of order four. In view of (5.24), we consider the following system of homological equations for $\mathcal{F}_{i j}^{(3)}$,

$$
\begin{aligned}
& \omega \cdot \partial_{\theta} \mathcal{F}_{j 0}^{(3)}(\theta)+\mathcal{P}_{j 0}^{(2)}(\theta)=\left\langle\mathcal{P}_{j 0}^{(2)}\right\rangle_{\theta}, \quad 1 \leq j \leq 3, \\
& \left(\omega \cdot \partial_{\theta}+\mathrm{i} \Omega_{\perp}\right) \mathcal{F}_{01}^{(3)}(\theta)+\mathcal{P}_{01}^{(2)}(\theta)=0, \quad\left(\omega \cdot \partial_{\theta}+\mathrm{i} \Omega_{\perp}\right) \mathcal{F}_{11}^{(3)}(\theta)+\mathcal{P}_{11}^{(2)}(\theta)=0, \\
& \left(\omega \cdot \partial_{\theta}+\mathrm{i} \Omega_{\perp}\right) \mathcal{F}_{21}^{(3)}(\theta)+\mathcal{P}_{21}^{(2)}(\theta)=0 .
\end{aligned}
$$

Since by assumption $\omega \in \Pi_{\gamma}, 0<\gamma<1$ (cf. (1.19)), we can apply Lemmata B.1, B.2, to conclude that the system $(5.25)$ has a unique solution $\mathcal{F}_{i j}^{(3)}$, satisfying the properties (5.21). The Hamiltonian $\mathcal{H}^{(3)}$ in (5.24) then reads

$$
\begin{aligned}
& \mathcal{H}^{(3)}=\mathcal{N}^{(3)}+\mathcal{K}, \quad \mathcal{N}^{(3)}:=\omega \cdot y+\varepsilon \widehat{\omega} \cdot y+\frac{1}{2}\left\langle D^{-1} \Omega_{\perp} w, w\right\rangle+Q(y), \quad \mathcal{K}:=\mathcal{P}_{2}^{(2)}+\mathcal{O}_{4} . \\
& \widehat{\omega}:=\left\langle\mathcal{P}_{10}\right\rangle_{\theta}+\varepsilon\left\langle\mathcal{P}_{10}^{(2)}\right\rangle_{\theta}, \quad Q(y):=\frac{1}{2} \Omega_{S_{+}} y \cdot y+\varepsilon\left\langle\mathcal{P}_{20}^{(2)}\right\rangle_{\theta}[y, y]+\left\langle\mathcal{P}_{30}^{(2)}\right\rangle_{\theta}[y, y, y] .
\end{aligned}
$$

Here we dropped the irrelevant constant term $\varepsilon\left\langle\mathcal{P}_{00}\right\rangle_{\theta}+\varepsilon^{2}\left\langle\mathcal{P}_{00}^{(1)}\right\rangle_{\theta}$ from the Hamiltonan $\mathcal{H}^{(3)}$ (cf. Remark 4.2). By (5.19), (5.26), the components of the Hamiltonian vector field $X_{\mathcal{H}^{(3)}}=\left(X_{\mathcal{H}^{(3)}}^{(\theta)}, X_{\mathcal{H}^{(3)}}^{(y)}, X_{\mathcal{H}^{(3)}}^{\perp}\right) \mathrm{read}$

$$
\begin{aligned}
& X_{\mathcal{H}^{(3)}}^{(\theta)}(\mathfrak{x})=-\omega-\varepsilon \widehat{\omega}-\nabla_{y} Q(y)-\nabla_{y} \mathcal{P}_{2}^{(2)}(\mathfrak{x})-\nabla_{y} \mathcal{O}_{4}(\mathfrak{x}) \\
& X_{\mathcal{H}^{(3)}}^{(y)}(\mathfrak{x})=\nabla_{\theta} \mathcal{P}_{2}^{(2)}(\mathfrak{x})+\nabla_{\theta} \mathcal{O}_{4}(\mathfrak{x}) \\
& X_{\mathcal{H}^{(3)}}^{\perp}(\mathfrak{x})=\mathrm{i} \Omega_{\perp} w+\partial_{x} \nabla_{\perp} \mathcal{P}_{2}^{(2)}(\mathfrak{x})+\partial_{x} \nabla_{\perp} \mathcal{O}_{4}(\mathfrak{x})
\end{aligned}
$$

Since $\mathcal{P}_{2}^{(2)}$ is a $C^{\infty}$-smooth and small of order three and $\mathcal{O}_{4}$ is small of order four, $\nabla_{\theta} \mathcal{P}_{2}^{(2)}$ is small of order three and $\nabla_{\theta} \mathcal{O}_{4}$ is small of order four, implying that

$$
X_{\mathcal{H}^{(3)}}^{(y)} \in C_{b}^{\infty}\left(\mathcal{V}^{\sigma_{N}}(\delta) \times\left[0, \varepsilon_{0}\right], \mathbb{R}^{S_{+}}\right) \text {small of order three }
$$

for some $\sigma_{N}>0$. Towards $X_{\mathcal{H}^{(3)}}^{(\theta)}$, note that $\nabla_{y} \mathcal{O}_{4}$ is small of order three and that $\nabla_{y} \mathcal{P}_{2}^{(2)}$ (cf. (5.19)) is small of order two and has the additional property of being at least quadratic with respect to $w$. Therefore

$$
\nabla_{y} \mathcal{P}_{2}^{(2)}(\mathfrak{x})+\nabla_{y} \mathcal{O}_{4}(\mathfrak{x})=\Upsilon_{2}^{(\theta)}(\theta)[w, w]+\Upsilon_{3}^{(\theta)}(\mathfrak{x})
$$


where

$$
\begin{aligned}
\Upsilon_{2}^{(\theta)} \in C^{\infty}\left(\mathbb{T}^{S_{+}}, \mathcal{B}_{2}\left(H_{\perp}^{\sigma_{N}}\left(\mathbb{T}_{1}\right), \mathbb{R}^{S_{+}}\right)\right), \\
\Upsilon_{3}^{(\theta)} \in C^{\infty}\left(\mathcal{V}^{\sigma_{N}}(\delta) \times\left[0, \varepsilon_{0}\right], \mathbb{R}^{S_{+}}\right) \text {small of order three }
\end{aligned}
$$

for some $\sigma_{N}>0$. For later use, we discuss the normal component $X_{\mathcal{K}}^{\perp}$ of the vector field $X_{\mathcal{K}}$. Since by $(5.19), \mathcal{P}_{2}^{(2)}=\varepsilon^{3} \mathcal{P}_{00}^{(2)}(\theta)+\left\langle\mathcal{P}_{02}^{(2)}(\theta, y)[w], w\right\rangle+\mathcal{P}_{03}^{(2)}(\theta)[w, w, w]$ (cf. Remark 5.1) one infers that

$$
X_{\mathcal{K}}^{\perp}(\mathfrak{x})=\partial_{x} \nabla_{\perp} \mathcal{P}_{2}^{(2)}(\mathfrak{x})+\partial_{x} \nabla_{\perp} \mathcal{O}_{4}(\mathfrak{x})=2 \partial_{x} \mathcal{P}_{02}^{(2)}(\theta, y)[w]+\Upsilon_{2}^{\perp}(\theta)[w, w]+\Upsilon_{3}^{\perp}(\mathfrak{x})
$$

where $\Upsilon_{3}^{\perp}(\mathfrak{x})$ is small of order three. Since $X_{\mathcal{F}(3)} \in \mathcal{O S}^{2}(N)$ and $\partial_{x} \nabla_{\perp} \mathcal{P}^{(2)}$ $=\mathcal{O B}^{2}(1, N)+\mathcal{O S}^{2}(N)\left(\right.$ cf. 5.16, 5.17) and in view of the definition of $\mathcal{O}_{4}$ (cf. (5.24)) it then follows from Lemma 3.17 that

$$
\begin{gathered}
\partial_{x} \mathcal{P}_{02}^{(2)}(\theta, y)[w]=\mathcal{O B}_{w}^{2}(1, N)+\mathcal{O S}_{w}^{2}(1, N), \\
\Upsilon \frac{1}{2}(\theta)[w, w]=\mathcal{O B}_{w w}^{2}(1, N)+\mathcal{O S}_{w w}^{2}(N), \quad \Upsilon_{3}^{\perp}(\mathfrak{x})=\mathcal{O} \mathcal{B}^{3}(1, N)+\mathcal{O S}^{3}(N) .
\end{gathered}
$$

Proof of Proposition 5.1.. We define $\Phi:=\Phi^{(1)} \circ \Phi^{(2)} \circ \Phi^{(3)}$ where $\Phi^{(1)}, \Phi^{(2)}, \Phi^{(3)}$ are the symplectic coordinate transformations, given in the paragraphs above. Using the properties (5.5), (5.13), (5.22) of $\Phi^{(1)}, \Phi^{(2)}$, and $\Phi^{(3)}$, respectively one shows that there exists an integer $s_{N}>0$ with the property that for any $s \geq s_{N}$ there exist $0<\delta \equiv$ $\delta(s, \gamma, N)<1$ and $0<\varepsilon_{0} \equiv \varepsilon_{0}(s, \gamma, N)<1$ so that (5.1) holds,

$$
\Phi^{ \pm 1} \in \mathcal{C}_{b}^{\infty}\left(\mathcal{V}^{s}(\delta) \times\left[0, \varepsilon_{0}\right], \mathcal{V}^{s}(2 \delta)\right), \quad \Phi^{ \pm 1}(\mathfrak{x})-\mathfrak{x} \quad \text { small of order one } .
$$

Since $\mathcal{K}=\mathcal{P}_{2}^{(2)}+\mathcal{O}_{4}$, the remaining statements of Proposition 5.1 then follow by (5.28) $-(5.31)$.

\section{Normalization Steps by Para-Differential Calculus}

The goal of this section is to normalize terms in the vector field $X_{\mathcal{K}}$, which are linear or quadratic in the variable $w$, where $X_{\mathcal{K}}$ denotes the Hamiltonian vector field of the Hamiltonian $\mathcal{K}$ of Proposition 5.1. This is achieved in three steps, described in the following three subsections, by using para-differential calculus.

6.1. Normalization of terms linear or quadratic in $w$. The aim of this subsection is to reduce to constant coefficients the terms in the normal component $X^{\perp} \equiv X_{\mathcal{H}_{3}}^{\perp}$ of the vector field $X \equiv X_{\mathcal{H}_{3}}$, which are linear and quadratic in $w$. Recall that such a reduction is needed since $\Pi_{\gamma}^{(3)}$ (cf. (1.20)) allows for a loss of derivatives in space.

By Proposition 5.1, $X^{\perp}$ is of the form

$$
X^{\perp}(\mathfrak{x})=X_{\mathcal{H}_{3}}^{\perp}(\mathfrak{x}) \stackrel{(5.3)}{=} \mathrm{i} \Omega_{\perp} w+X_{\mathcal{K}}^{\perp}(\mathfrak{x}) .
$$

Since $\Omega_{\perp}$ is a diagonal Fourier multiplier with constant real coefficients (cf. (1.18), (1.42)), it remains to normalize $X_{\mathcal{K}}^{\perp}(\mathfrak{x})$ in the above sense. 
By Proposition 5.1, $X_{\mathcal{K}}^{\perp}(\mathfrak{x})$ admits an expansion of the form

$$
\begin{gathered}
X_{\mathcal{K}}^{\perp}(\mathfrak{x})=X_{1}^{\perp}(\theta, y)[w]+X_{2}^{\perp}(\theta)[w, w]+\mathcal{O B}^{3}(1, N)+\mathcal{O S}^{3}(1, N), \\
X_{1}^{\perp}(\theta, y)[w]:=\Upsilon_{1}^{\perp}(\theta, y)[w]+\mathcal{R}_{N, 1}^{\perp}(\theta, y)[w], \\
X_{2}^{\perp}(\theta)[w, w]:=\Upsilon_{2}^{\perp}(\theta, w)[w]+\mathcal{R}_{N, 2}^{\perp}(\theta)[w, w],
\end{gathered}
$$

where

$$
\begin{aligned}
& \Upsilon_{1}^{\perp}(\theta, y)[w]=\Pi_{\perp} \sum_{k=0}^{N+1} T_{a_{1-k}(\theta, y)} \partial_{x}^{1-k} w \in \mathcal{O B}_{w}^{2}(1, N), \quad \mathcal{R}_{N, 1}^{\perp}(\theta, y)[w] \in \mathcal{O S}_{w}^{2}(N) \\
& \Upsilon_{2}^{\perp}(\theta, w)[w]=\Pi_{\perp} \sum_{k=0}^{N+1} T_{A_{1-k}(\theta)[w]} \partial_{x}^{1-k} w \in \mathcal{O B}_{w w}^{2}(1, N), \quad \mathcal{R}_{N, 2}^{\perp}(\theta)[w, w] \in \mathcal{O S}_{w w}^{2}(N) .
\end{aligned}
$$

By Definition 3.4, for any given $N \in \mathbb{N}$, there are integers $s_{N}, \sigma_{N}>0$ (large) with the property that for any $s \geq s_{N}$ there exist $0<\delta=\delta(s, \gamma, N)<1$ and $0<\varepsilon_{0} \equiv$ $\varepsilon_{0}(s, \gamma, N)<1$ so that for any $0 \leq k \leq N+1$

$$
\begin{aligned}
& a_{1-k} \in C_{b}^{\infty}\left(\mathbb{T}^{S_{+}} \times B_{S_{+}}(\delta) \times\left[0, \varepsilon_{0}\right], H^{s}\left(\mathbb{T}_{1}\right)\right) \text { small of order one, } \\
& A_{1-k} \in C^{\infty}\left(\mathbb{T}^{S_{+}} \times\left[0, \varepsilon_{0}\right], \mathcal{B}\left(H_{\perp}^{s+\sigma_{N}}\left(\mathbb{T}_{1}\right), H^{S}\left(\mathbb{T}_{1}\right)\right)\right) .
\end{aligned}
$$

Note that $X_{1}^{\perp}(\theta, y)[w]$ is a vector field small of order 2 and linear in $w$, whereas $X_{2}^{\perp}(\theta)[w, w]$ is small of order 2, but quadratic in $w$. Since the vector field $X_{\mathcal{K}}^{\perp}$ is Hamiltonian, every term in the expansion (6.1), which is homogeneous in the coordinates $y, w$, is a Hamiltonian vector field as well. In particular, $X_{1}^{\perp}(\theta, y)[w]$ is such a vector field.

Preliminary analysis of the vector field $X_{1}^{\perp}(\theta, y)[w]$. Since $X_{1}^{\perp}(\theta, y)[w]$ is a Hamiltonian vector field which is linear in $w,($ A.2) in Appendix A implies that the diagonal operator

$$
\operatorname{diag}_{j \in S^{\perp}}\left[X_{1}^{\perp}(\theta, y)\right]_{j}^{j}
$$

is skew-adjoint,

$$
\left[X_{1}^{\perp}(\theta, y)\right]_{j}^{j}=-\overline{\left[X_{1}^{\perp}(\theta, y)\right]_{j}^{j}}, \quad j \in S^{\perp} .
$$

We will show that the normal form transformations, constructed in this and the following subsection, preserve this property of $X_{1}^{\perp}(\theta, y)[w]$. Since this is the only property of the transformed vector field $X_{1}^{\perp}(\theta, y)[w]$ which is needed in the energy estimates in Sect. 7 we can allow for normal form transformations, which are not necessarily symplectic, as long as they preserve (6.5).

Our aim is to construct iteratively a coordinate transformation on $\mathcal{V}^{s}(\delta)$ so that when expressed in the new coordinates, the vector field $X_{1}^{\perp}(\theta, y)[w]+X_{2}^{\perp}(\theta)[w, w]$ is again of the form (6.2) and that the coefficients $a_{1-k}(\theta, y)+A_{1-k}(\theta)[w], 0 \leq k \leq N+1$, are independent of $x$. At the $(n+1)$ th step, $n \geq 0$, we deal with a vector field $X_{n}=$ $\left(X_{n}^{(\theta)}, X_{n}^{(y)}, X_{n}^{\perp}\right)$, defined as the pull back of $X$ by the composition of the transformations up to the nth step, of the form 


$$
\begin{aligned}
X_{n}^{(\theta)}(\mathfrak{x})= & -\omega-\varepsilon \widehat{\omega}-\nabla_{y} Q(y)-\Upsilon_{2}^{(\theta)}(\theta)[w, w]+\mathcal{O}_{3}^{(\theta)}(\mathfrak{x}), \quad X_{n}^{(y)}(\mathfrak{x})=\mathcal{O}_{3}^{(y)}(\mathfrak{x}), \\
X_{n}^{\perp}(\mathfrak{x})= & \mathrm{i} \Omega_{\perp} w+\mathcal{D}_{n, 1}^{\perp}(\theta, y)[w]+\mathcal{D}_{n, 2}^{\perp}(\theta, w)[w]+X_{n, 1}^{\perp}(\theta, y)[w]+X_{n, 2}^{\perp}(\theta, w)[w] \\
& +\mathcal{R}_{N, 1}^{\perp}(\theta, y)[w]+\mathcal{R}_{N, 2}^{\perp}(\theta, w)[w]+\mathcal{O B}^{3}(1, N)+\mathcal{O S}^{3}(N)
\end{aligned}
$$

where for notational convenience, we write $\mathcal{R}_{N, j}^{\perp} \equiv \mathcal{R}_{n, N, j}^{\perp}$ for $j=1,2$, and where

$$
\begin{array}{ll}
\mathcal{D}_{n, 1}^{\perp}(\theta, y)[w] \in \mathcal{O} \mathcal{F}_{w}^{2}(1, N), & \mathcal{D}_{n, 2}^{\perp}(\theta, w)[w] \in \mathcal{O F}_{w w}^{2}(1, N), \\
X_{n, 1}^{\perp}(\theta, y)[w] \in \mathcal{O B}_{w}^{2}(1-n, N), & X_{n, 2}^{\perp}(\theta, w)[w] \in \mathcal{O} \mathcal{B}_{w w}^{2}(1-n, N) \\
\mathcal{R}_{N, 1}^{\perp}(\theta, y)[w] \in \mathcal{O S}_{w}^{2}(N), & \mathcal{R}_{N, 2}^{\perp}(\theta, w)[w] \in \mathcal{O S}_{w w}^{2}(N), \\
\mathcal{O}_{3}^{(\theta)}, \mathcal{O}_{3}^{(y)} \in C_{b}^{\infty}\left(\mathcal{V}^{\sigma_{N}}(\delta) \times\left[0, \varepsilon_{0}\right],\right. & \left.\mathbb{R}^{S_{+}}\right) \text {small of order three }
\end{array}
$$

for some $\sigma_{N}>0$. Moreover

$$
\begin{gathered}
\mathcal{D}_{n, 1}^{\perp}(\theta, y)=-\mathcal{D}_{n, 1}^{\perp}(\theta, y)^{\top}, \quad\left[X_{n, 1}^{\perp}(\theta, y)\right]_{j}^{j}=-\overline{\left[X_{n, 1}^{\perp}(\theta, y)\right]_{j}^{j}}, \\
{\left[\mathcal{R}_{N, 1}^{\perp}(\theta, y)\right]_{j}^{j}=-\overline{\left[\mathcal{R} \stackrel{\perp}{N}, 1^{\perp}(\theta, y)\right]_{j}^{j}}, \quad \forall j \in S^{\perp} .}
\end{gathered}
$$

Our goal at the $(n+1)$ th step is to construct a transformation so that when expressed in the new coordinates, the vector field $X_{n, 1}^{\perp}(\theta, y)[w]+X_{n, 2}^{\perp}(\theta, w)[w]$ is of order $1-(n+1)=$ $-n$. Since $X_{n, 1}^{\perp}(\theta, y)[w] \in \mathcal{O B}_{w}^{2}(1-n, N)$ and $X_{n, 2}^{\perp}(\theta, w)[w] \in \mathcal{O B}_{w w}^{2}(1-n, N)$ we can write

$$
\begin{aligned}
& X_{n, 1}^{\perp}(\theta, y)[w]=\Pi_{\perp} T_{a_{1-n}(\theta, y)} \partial_{x}^{1-n} w+\mathcal{O B}_{w}^{2}(-n, N), \\
& X_{n, 1}^{\perp}(\theta, w)[w]=\Pi_{\perp} T_{A_{1-n}(\theta)[w]} \partial_{x}^{1-n} w+\mathcal{O} \mathcal{B}_{w w}^{2}(-n, N)
\end{aligned}
$$

with the property that there are integers $s_{N}>0, \sigma_{N} \geq 0$ so that for any $s \geq s_{N}$ there exist $0<\delta \equiv \delta(s, \gamma, N)<1$ and $0<\varepsilon_{0} \equiv \varepsilon_{0}(s, \gamma, N)<1$ so that

$$
\begin{aligned}
& a_{1-n} \in C_{b}^{\infty}\left(\mathbb{T}^{S_{+}} \times B_{S_{+}}(\delta) \times\left[0, \varepsilon_{0}\right], H^{s}\left(\mathbb{T}_{1}\right)\right) \text { small of order one, } \\
& A_{1-n} \in C^{\infty}\left(\mathbb{T}^{S_{+}} \times\left[0, \varepsilon_{0}\right], \mathcal{B}\left(H_{\perp}^{s+\sigma_{N}}\left(\mathbb{T}_{1}\right), H^{s}\left(\mathbb{T}_{1}\right)\right)\right) .
\end{aligned}
$$

Hence we need to normalize the vector field $\Pi_{\perp} T_{a_{1-n}(\theta, y)+A_{1-n}(\theta)[w]} \partial_{x}^{1-n} w$. In order to achieve this, we consider a para-differential vector field of the form

$$
\begin{aligned}
& Y_{n}^{\perp}(\theta, y, w)=Y_{n, 1}^{\perp}(\theta, y)[w]+Y_{n, 2}^{\perp}(\theta, w)[w], \\
& Y_{n, 1}^{\perp}(\theta, y)[w]:=\Pi_{\perp} T_{b_{n}(\theta, y)} \partial_{x}^{-n-1} w \in \mathcal{O B}_{w}^{2}(-n-1, N), \\
& Y_{n, 2}^{\perp}(\theta, w)[w]:=\Pi_{\perp} T_{B_{n}(\theta)[w]} \partial_{x}^{-n-1} w \in \mathcal{O B}_{w w}^{2}(-n-1, N),
\end{aligned}
$$

and make the ansatz that $b_{n}(\theta, y), B_{n}(\theta)[w]$ are smooth functions (satisfying conditions as in (6.10)) and

$$
\left\langle b_{n}(\theta, y)\right\rangle_{x}=0, \quad\left\langle B_{n}(\theta)[w]\right\rangle_{x}=0 .
$$

To determine $b_{n}$ and $B_{n}$, we compute the pullback $X_{n+1}:=\Phi_{Y_{n}}^{*} X_{n}$ of $X_{n}$ by the time one flow map $\Phi_{Y_{n}}$. corresponding to the vector field $Y_{n}$. By Lemmata 3.7, 3.10, 
3.13 and the induction hypothesis (6.8), one infers that the components of $X_{n+1}=$ $\left(X_{n+1}^{(\theta)}, X_{n+1}^{(y)}, X_{n+1}^{\perp}\right)$ satisfy

$$
\begin{aligned}
X_{n+1}^{(\theta)}(\mathfrak{x})= & -\omega-\varepsilon \widehat{\omega}-\nabla_{y} Q(y)-\Upsilon_{2}^{(\theta)}(\theta)[w, w]+\mathcal{O}_{3}^{(\theta)}(\mathfrak{x}), \quad X_{n+1}^{(y)}(\mathfrak{x})=\mathcal{O}_{3}^{(y)}(\mathfrak{x}), \\
X_{n+1}^{\perp}(\mathfrak{x})= & \mathrm{i} \Omega_{\perp} w+\mathcal{D}_{n, 1}^{\perp}(\theta, y)[w]+\mathcal{D}_{n, 2}^{\perp}(\theta, w)[w]+\Pi_{\perp} T_{-3 \partial_{x} b_{n}(\theta, y)+a_{1-n}(\theta, y)} \partial_{x}^{1-n} w \\
& +\Pi_{\perp} T_{-3 \partial_{x} B_{n}(\theta)[w]+A_{1-n}(\theta)[w]} \partial_{x}^{1-n} w+X_{n+1,1}^{\perp}(\theta, y)[w]+\mathcal{R}_{N, 1}^{\perp}(\theta, y)[w] \\
& +X_{n+1,2}^{\perp}(\theta, w)[w]+\mathcal{R}_{N, 2}^{\perp}(\theta)[w, w]+\mathcal{O B}^{3}(1, N)+\mathcal{O S}^{3}(N)
\end{aligned}
$$

where

$$
\begin{array}{ll}
X_{n+1,1}^{\perp}(\theta, y)[w] \in \mathcal{O B}_{w}^{2}(-n, N), & X_{n+1,2}^{\perp}(\theta, w)[w] \in \mathcal{O B}_{w w}^{2}(-n, N) \\
\mathcal{R}_{N, 1}^{\perp}(\theta, y)[w] \in \mathcal{O S}_{w}^{2}(N), & \mathcal{R}_{N, 2}^{\perp}(\theta, w)[w] \in \mathcal{O S}_{w w}^{2}(N), \\
\mathcal{O}_{3}^{(\theta)}, \mathcal{O}_{3}^{(y)} \in C_{b}^{\infty}\left(\mathcal{V}^{\sigma}(\delta) \times\left[0, \varepsilon_{0}\right],\right. & \left.\mathbb{R}^{S_{+}}\right) \text {small of order three }
\end{array}
$$

and the diagonal matrix elements of the operators $X_{n+1,1}^{\perp}(\theta, y), \mathcal{R}_{N, 1}^{\perp}(\theta, y)$ are purely imaginary, namely

$$
\left[X_{n+1,1}^{\perp}(\theta, y)\right]_{j}^{j},\left[\mathcal{R}_{N, 1}^{\perp}(\theta, y)\right]_{j}^{j} \in \mathrm{i} \mathbb{R}, \quad \forall j \in S^{\perp} .
$$

We then choose $b_{n}(\theta, y)$ and $B_{n}(\theta)[w]$ to be solutions of

$$
\begin{aligned}
& -3 \partial_{x} b_{n}(\theta, y)+a_{1-n}(\theta, y)=\left\langle a_{1-n}(\theta, y)\right\rangle_{x}, \\
& -3 \partial_{x} B_{n}(\theta)[w]+A_{1-n}(\theta)[w]=\left\langle A_{1-n}(\theta)[w]\right\rangle_{x} .
\end{aligned}
$$

More precisely, we define

$$
\begin{aligned}
& b_{n}(\theta, y):=\frac{1}{3} \partial_{x}^{-1}\left(a_{1-n}(\theta, y)-\left\langle a_{1-n}(\theta, y)\right\rangle_{x}\right), \\
& B_{n}(\theta)[w]:=\frac{1}{3} \partial_{x}^{-1}\left(A_{1-n}(\theta)[w]-\left\langle A_{1-n}(\theta)[w]\right\rangle_{x}\right) .
\end{aligned}
$$

Since $\Pi_{\perp} T_{\left\langle a_{1-n}(\theta, y)\right\rangle_{x}} \partial_{x}^{1-n} w=\left\langle a_{1-n}(\theta, y)\right\rangle_{x} \partial_{x}^{1-n} w$ and

$$
\Pi_{\perp} T_{\left\langle A_{1-n}(\theta)[w]\right\rangle_{x}} \partial_{x}^{1-n} w=\left\langle A_{1-n}(\theta)[w]\right\rangle_{x} \partial_{x}^{1-n} w
$$

one infers from (6.10) that

$$
\begin{aligned}
& \mathcal{D}_{n+1,1}^{\perp}(\theta, y)[w]:=\mathcal{D}_{n, 1}^{\perp}(\theta, y)[w]+\left\langle a_{1-n}(\theta, y)\right\rangle_{x} \partial_{x}^{1-n} w \in \mathcal{O F}_{w}^{2}(1, N), \\
& \mathcal{D}_{n+1,2}^{\perp}(\theta, w)[w]:=\mathcal{D}_{n, 2}^{\perp}(\theta, w)[w]+\left\langle A_{1-n}(\theta)[w]\right\rangle_{x} \partial_{x}^{1-n} w \in \mathcal{O F}_{w w}^{2}(1, N) .
\end{aligned}
$$

Since $a_{1-n}(\theta, y)$ is real valued, the Fourier multiplier $\left\langle a_{1-n}(\theta, y)\right\rangle_{x} \partial^{1-n}$ is skew-adjoint if $n$ is even. Futhermore, by the induction hypothesis (6.8) and Lemmata A.1, A.2 in Appendix A, one has

$$
\left\langle a_{1-n}(\theta, y)\right\rangle_{x}=0 \text { if } n \text { is odd. }
$$

Hence the Fourier multiplier $\mathcal{D}_{n+1,1}^{\perp}(\theta, y)$ is skew-adjoint. Altogether we showed that the vector field $X_{n+1}^{\perp}$ is of the form 


$$
\begin{aligned}
X_{n+1}^{\perp}(\mathfrak{x})= & \mathrm{i} \Omega_{\perp} w+\mathcal{D}_{n+1,1}^{\perp}(\theta, y)[w]+\mathcal{D}_{n+1,2}^{\perp}(\theta, w)[w]+X_{n+1,1}^{\perp}(\theta, y)[w] \\
& +X_{n+1,2}^{\perp}(\theta, w)[w]+\mathcal{R}_{N, 1}^{\perp}(\theta, y)[w]+\mathcal{R}_{N, 2}^{\perp}(\theta, w)[w]+\mathcal{O B}^{3}(1, N)+\mathcal{O S}^{3}(N) .
\end{aligned}
$$

We thus have proved the following

Proposition 6.1. For any $N \in \mathbb{N}$, there exist $s_{N}, \sigma_{N}>0$ with the following property: for any $s \geq s_{N}$ there exist $0<\delta \equiv \delta(s, \gamma, N)<1,0<\varepsilon_{0} \equiv \varepsilon_{0}(s, \gamma, N)<1$ so that the following holds: there exists a transformation $\Psi^{(1)}$ with inverse $\left(\Psi^{(1)}\right)^{-1}$ (cf. Remark 3.4),

$$
\left(\Psi^{(1)}\right)^{ \pm 1} \in \mathcal{C}_{b}^{\infty}\left(\mathcal{V}^{s}(\delta) \times\left[0, \varepsilon_{0}\right], \mathcal{V}^{s}(2 \delta)\right), \quad \forall s \geq s_{N}, \quad\left(\Psi^{(1)}\right)^{ \pm 1}(\mathfrak{x})-\mathfrak{x} \text { small of order two },
$$

so that the transformed vector field $X_{4}:=\left(\Psi^{(1)}\right)^{*} X_{\mathcal{H}_{3}}=\left(X_{4}^{(\theta)}, X_{4}^{(y)}, X_{4}^{\perp}\right)$ has the following properties:

$$
\begin{aligned}
X_{4}^{(\theta)}(\mathfrak{x})= & -\omega-\varepsilon \widehat{\omega}-\nabla_{y} Q(y)-\Upsilon_{2}^{(\theta)}(\theta)[w, w]+\mathcal{O}_{3}^{(\theta)}(\mathfrak{x}), \quad X_{4}^{(y)}(\mathfrak{x})=\mathcal{O}_{3}^{(y)}(\mathfrak{x}), \\
X_{4}^{\perp}(\mathfrak{x})= & \mathrm{i} \Omega_{\perp} w+\mathcal{D}_{4,1}^{\perp}(\theta, y)[w]+\mathcal{D}_{4,2}^{\perp}(\theta, w)[w]+\mathcal{R}_{N, 1}^{\perp}(\theta, y)[w]+\mathcal{R}_{N, 2}^{\perp}(\theta, w)[w] \\
& +\mathcal{O B}^{3}(1, N)+\mathcal{O S}^{3}(N)
\end{aligned}
$$

where

$$
\begin{array}{ll}
\mathcal{D}_{4,1}^{\perp}(\theta, y)[w] \in \mathcal{O} \mathcal{F}_{w}^{2}(1, N), & \mathcal{D}_{4,2}^{\perp}(\theta, w)[w] \in \mathcal{O F}_{w w}^{2}(1, N), \\
\mathcal{R}_{N, 1}^{\perp}(\theta, y)[w] \in \mathcal{O S}_{w}^{2}(N), & \mathcal{R}_{N, 2}^{\perp}(\theta, w)[w] \in \mathcal{O S}_{w w}^{2}(N), \\
\mathcal{O}_{3}^{(\theta)}, \mathcal{O}_{3}^{(y)} \in C_{b}^{\infty}\left(\mathcal{V}^{\sigma_{N}}(\delta) \times\left[0, \varepsilon_{0}\right], \mathbb{R}^{S_{+}}\right) & \text {small of order three. }
\end{array}
$$

Moreover

$$
\mathcal{D}_{4,1}^{\perp}(\theta, y)=-\left(\mathcal{D}_{4,1}^{\perp}(\theta, y)\right)^{\top}, \quad\left[\mathcal{R}_{N, 1}^{\perp}(\theta, y)\right]_{j}^{j} \in \mathrm{i} \mathbb{R}, \quad \forall j \in S^{\perp} .
$$

6.2. Normalization of Fourier multiplier quadratic in $w$. The goal of this subsection is to normalize the vector field $\mathcal{D}_{4,2}^{\perp}(\theta, w)[w]$ in (6.20). According to Proposition 6.1 and Definitions (3.2), (3.4),

$$
\begin{aligned}
& \mathcal{D}_{4,2}^{\perp}(\theta, w)[w]=\Lambda_{1}^{\perp}(\theta)[w] \partial_{x} w+\widetilde{\mathcal{D}}_{4,2}^{\perp}(\theta, w)[w], \\
& \widetilde{\mathcal{D}}_{4,2}^{\perp}(\theta, w)[w]:=\sum_{k=1}^{N+1} \Lambda_{1-k}^{\perp}(\theta)[w] \partial_{x}^{1-k} w \in \mathcal{O F}_{w w}^{2}(0, N),
\end{aligned}
$$

where for any $0 \leq k \leq N+1, \Lambda_{1-k}^{\perp} \in C^{\infty}\left(\mathbb{T}^{S_{+}}, \mathcal{B}\left(H_{\perp}^{\sigma_{N}}, \mathbb{R}\right)\right)$ for some $\sigma_{N}>0$ (large). Since $\Lambda_{1}(\theta)[w]$ is real valued, the leading order operator $\Lambda_{1}(\theta)[w] \partial_{x}$ is a skew-adjoint Fourier multiplier and hence has the property needed for the energy estimates in Sect. 7. This however is not true for $\widetilde{\mathcal{D}}_{4,2}^{\perp}(\theta, w)[w]$. The goal of this section is to eliminate it. To this end, we consider a vector field of the form

$$
\mathcal{M}(\mathfrak{x}):=\left(0,0, \mathcal{M}^{\perp}(\theta, w)[w]\right), \quad \mathcal{M}^{\perp}(\theta, w)[w]=\sum_{k=1}^{N+1} \Xi_{1-k}^{\perp}(\theta)[w] \partial_{x}^{1-k} w \in \mathcal{O} \mathcal{F}_{w w}^{2}(0, N)
$$


where $\Xi_{1-k}^{\perp}(\theta)$ will be chosen so that the time one flow map $\Phi_{\mathcal{M}}$, generated by the vector field $X_{\mathcal{M}}$, is a coordinate transformation serving our needs. In more detail, consider the pullback $X_{5}:=\Phi_{\mathcal{M}}^{*} X_{4}=\left(X_{5}^{(\theta)}, X_{5}^{(y)}, X_{5}^{\perp}\right)$ of the vector field $X_{4}$ of Proposition 6.1 by $\Phi_{\mathcal{M}}$. By Lemmata $3.13,3.15$, one has

$$
\begin{aligned}
X_{5}^{(\theta)}(\mathfrak{x})= & -\omega-\varepsilon \widehat{\omega}-\nabla_{y} Q(y)-\Upsilon_{2}^{(\theta)}(\theta)[w, w]+\mathcal{O}_{3}^{(\theta)}(\mathfrak{x}), \quad X_{5}^{(y)}=\mathcal{O}_{3}^{(y)}(\mathfrak{x}) \\
X_{5}^{\perp}(\mathfrak{x})= & \mathrm{i} \Omega_{\perp} w+\mathcal{D}_{4,1}^{\perp}(\theta, y)[w]+\Lambda_{1}(\theta)[w] \partial_{x} w+\mathcal{R}_{4,1}^{\perp}(\theta, y)[w]+\mathcal{R}_{4,2}^{\perp}(\theta, w)[w] \\
& +\omega \cdot \partial_{\theta} \mathcal{M}^{\perp}(\theta, w)[w]-\mathcal{M}^{\perp}\left(\theta, \mathrm{i} \Omega_{\perp} w\right)[w]+\widetilde{\mathcal{D}}_{4,2}(\theta, w)[w] \\
& +\mathcal{O B}^{3}(1, N)+\mathcal{O S}^{3}(N)
\end{aligned}
$$

where for some integer $\sigma_{N}>0, \mathcal{O}_{3}^{(\theta)}, \mathcal{O}_{3}^{(y)}$ are in $C_{b}^{\infty}\left(\mathcal{V}^{\sigma_{N}}(\delta) \times\left[0, \varepsilon_{0}\right], \mathbb{R}^{S_{+}}\right)$and small of order three. The vector field $\mathcal{M}^{\perp}(\theta, w)[w]$ is chosen to be a solution the following homological equation

$$
\omega \cdot \partial_{\theta} \mathcal{M}^{\perp}(\theta, w)[w]-\mathcal{M}^{\perp}\left(\theta, \mathrm{i} \Omega_{\perp} w\right)[w]+\widetilde{\mathcal{D}}_{4,2}^{\perp}(\theta, w)[w]=0,
$$

or in view of (6.23), (6.24) equivalently, that for any $1 \leq k \leq N+1, \Xi_{1-k}^{\perp}(\theta)[w]$ is a solution of

$$
\omega \cdot \partial_{\theta} \Xi_{1-k}^{\perp}(\theta)[w]-\Xi_{1-k}^{\perp}(\theta)\left[i \Omega_{\perp} w\right]+\Lambda_{1-k}^{\perp}(\theta)[w]=0 .
$$

Since $\Lambda_{1-k}^{\perp}, \Xi_{1-k}^{\perp} \in C^{\infty}\left(\mathbb{T}^{S_{+}} \times\left[0, \varepsilon_{0}\right], \mathcal{B}\left(H_{\perp}^{\sigma_{N}}\left(\mathbb{T}_{1}\right), \mathbb{R}\right)\right)$, there exist uniquely determined maps $a_{\Lambda_{1-k}^{\perp}}, a_{\Xi_{1-k}^{\perp}}$ in $C^{\infty}\left(\mathbb{T}^{S_{+}} \times\left[0, \varepsilon_{0}\right], H_{\perp}^{-\sigma_{N}}\left(\mathbb{T}_{1}\right)\right)$ so that

$$
\Lambda_{1-k}^{\perp}(\theta)[w]=\left\langle a_{\Lambda_{1-k}^{\perp}}(\theta), w\right\rangle, \quad \Xi_{1-k}^{\perp}(\theta)[w]=\left\langle a_{\Xi_{1-k}^{\perp}}(\theta), w\right\rangle .
$$

Equation (6.27) then reads

$$
\left\langle\omega \cdot \partial_{\theta} a_{\Xi_{1-k}^{\perp}}(\theta), w\right\rangle-\left\langle a_{\Xi_{1-k}^{\perp}}(\theta), \mathrm{i} \Omega_{\perp} w\right\rangle+\left\langle a_{\Lambda_{1-k}^{\perp}}(\theta), w\right\rangle=0 .
$$

Since $\mathrm{i} \Omega_{\perp}$ is skew-adjoint, one has $-\left\langle a_{\Xi_{1-k}^{\perp}}(\theta), \mathrm{i} \Omega_{\perp} w\right\rangle=\left\langle\mathrm{i} \Omega_{\perp} a_{\Xi_{1-k}^{\perp}}(\theta), w\right\rangle$. We choose $a_{\Xi_{1-k}^{\perp}}$ as the solution of

$$
\left(\omega \cdot \partial_{\theta}+\mathrm{i} \Omega_{\perp}\right) a_{\Xi_{1-k}^{\perp}}(\theta)+a_{\Lambda_{1-k}^{\perp}}(\theta)=0
$$

This equation can be solved by expanding $a_{\Xi_{1-k}^{\perp}}(\theta)$ and $a_{\Lambda_{1-k}^{\perp}}(\theta)$ in Fourier series with respect to $\theta$ and $x$,

$$
\begin{aligned}
& a_{\Xi_{1-k}^{\perp}}(\theta)=\sum_{(\ell, j) \in \mathbb{Z}^{S_{+}} \times S^{\perp}} \widehat{a}_{\Xi_{1-k}^{\perp}}(\ell, j) e^{\mathrm{i} \ell \cdot \theta} e^{\mathrm{i} 2 \pi j x}, \\
& a_{\Lambda_{1-k}^{\perp}}(\theta)=\sum_{(\ell, j) \in \mathbb{Z}^{S_{+}} \times S^{\perp}} \widehat{a}_{\Lambda_{1-k}^{\perp}}(\ell, j) e^{\mathrm{i} \ell \cdot \theta} e^{\mathrm{i} 2 \pi j x} .
\end{aligned}
$$


Since by assumption $\omega \in \Pi_{\gamma}^{(1)}, 0<\gamma<1$, (cf. (1.20)), Eq. (6.29) can be solved. The solution $a_{\Xi_{1-k}^{\perp}}(\theta)$ is given by

$$
a_{\Xi_{1-k}^{\perp}}=-\left(\omega \cdot \partial_{\theta}+\mathrm{i} \Omega_{\perp}\right)^{-1} a_{\Lambda_{1-k}^{\perp}}=-\sum_{(\ell, j) \in \mathbb{Z}^{S_{+} \times S^{\perp}}} \frac{\widehat{a}_{\Lambda_{1-k}^{\perp}}(\ell, j)}{\mathrm{i}\left(\omega \cdot \ell+\Omega_{j}\right)} e^{\mathrm{i} \ell \cdot \theta} e^{\mathrm{i} 2 \pi j x} .
$$

Since $a_{\Lambda_{1-k}^{\perp}} \in C^{\infty}\left(\mathbb{T}^{S_{+}}, H_{\perp}^{-\sigma_{N}}\left(\mathbb{T}_{1}\right)\right)$, one infers that $a_{\Xi_{1-k}^{\perp}} \in C^{\infty}\left(\mathbb{T}^{S_{+}}, H_{\perp}^{-\sigma_{N}-\tau}\left(\mathbb{T}_{1}\right)\right)$ and therefore (6.24) is verified and Eq. (6.26) is solved. Finally, the vector field $X_{5}^{\perp}$ is of the form

$$
\begin{aligned}
& X_{5}^{\perp}(\mathfrak{x})= \mathrm{i} \Omega_{\perp} w+\mathcal{D}_{5}(\mathfrak{x})[w]+\mathcal{R}_{N, 1}^{\perp}(\theta, y)[w]+\mathcal{R}_{N, 2}^{\perp}(\theta, w)[w] \\
&+\mathcal{O B}^{3}(1, N)+\mathcal{O S}^{3}(N), \\
& \mathcal{D}_{5}^{\perp}(\mathfrak{x})[w]:=\mathcal{D}_{4,1}^{\perp}(\theta, y)[w]+\Lambda_{1}^{\perp}(\theta)[w] \partial_{x} w \in \mathcal{O F}^{2}(1, N),
\end{aligned}
$$

where the remainders $\mathcal{R}_{N, 1}^{\perp}(\theta, y)[w], \mathcal{R}_{N, 2}^{\perp}(\theta, w)[w]$ are given in Proposition 6.1. Furthermore, $\mathcal{D} \frac{\perp}{5}(\mathfrak{x})$ is skew-adjoint,

$$
\mathcal{D}_{5}^{\perp}(\mathfrak{x})^{\top}=-\mathcal{D}_{5}^{\perp}(\mathfrak{x})
$$

We summarize our findings of this subsection as follows.

Proposition 6.2. For any $N \in \mathbb{N}$, there exists an integer $s_{N}>0$ with the property that for any $s \geq s_{N}$ there exist $0<\delta \equiv \delta(s, \gamma, N)<1$ and $0<\varepsilon_{0} \equiv \varepsilon_{0}(s, \gamma, N)<1$ so that the following holds: there exists a transformation $\Psi^{(2)}$ with inverse $\left(\Psi^{(2)}\right)^{-1}$ (cf. Remark 3.4),

$$
\left(\Psi^{(2)}\right)^{ \pm 1} \in \mathcal{C}_{b}^{\infty}\left(\mathcal{V}^{s}(\delta) \times\left[0, \varepsilon_{0}\right], \mathcal{V}^{s}(2 \delta)\right), \quad \forall s \geq s_{N}, \quad\left(\Psi^{(2)}\right)^{ \pm 1}(\mathfrak{x})-\mathfrak{x} \text { small of order two }
$$

so that the transformed vector field $X_{5}:=\left(\Psi^{(2)}\right)^{*} X_{4}=\left(X_{5}^{(\theta)}, X_{5}^{(y)}, X_{5}^{\perp}\right)$ has the form

$$
\begin{aligned}
X_{5}^{(\theta)}(\mathfrak{x}) & =-\omega-\varepsilon \widehat{\omega}-\nabla_{y} Q(y)-\Upsilon_{2}^{(\theta)}(\theta)[w, w]+\mathcal{O}_{3}(\mathfrak{x}), \quad X_{5}^{(y)}(\mathfrak{x})=\mathcal{O}_{3}(\mathfrak{x}), \\
X_{5}^{\perp}(\mathfrak{x}) & =\mathrm{i} \Omega_{\perp} w+\mathcal{D}_{5}^{\perp}(\mathfrak{x})[w]+\mathcal{R}_{N, 1}^{\perp}(\theta, y)[w]+\mathcal{R}_{N, 2}^{\perp}(\theta, w)[w]+\mathcal{O} \mathcal{B}^{3}(1, N)+\mathcal{O} \mathcal{S}^{3}(N)
\end{aligned}
$$

where

$$
\mathcal{D}_{5}^{\perp}(\mathfrak{x})[w] \in \mathcal{O} \mathcal{F}^{2}(1, N), \quad \mathcal{D}_{5}^{\perp}(\mathfrak{x})=-\mathcal{D}_{5}^{\perp}(\mathfrak{x})^{\top}
$$

and the smoothing remainders $\mathcal{R}_{N, 1}^{\perp}(\theta, y)[w], \mathcal{R}_{N, 2}^{\perp}(\theta, w)$ [w] are given by Proposition 6.1.

6.3. Normalization of the smoothing remainders. In this subsection, we normalize the vector field 


$$
\left(\Upsilon_{2}^{(\theta)}(\theta)[w, w], 0, \mathcal{R}_{N, 1}^{\perp}(\theta, y)[w]+\mathcal{R}_{N, 2}^{\perp}(\theta)[w, w]\right),
$$

which is part of the vector field $X_{5}$ defined in (6.34). Note that all the terms are either linear or quadratic in the variable $w$. We consider a smoothing vector field of the form

$$
\mathcal{S}(\mathfrak{x}):=\left(\mathcal{S}^{(\theta)}(\theta)[w, w], 0, \mathcal{S}_{1}^{\perp}(\theta, y)[w]+\mathcal{S}_{2}^{\perp}(\theta)[w, w]\right)
$$

where we make the ansatz that for some $\sigma_{N}>0, \mathcal{S}^{(\theta)} \in C^{\infty}\left(\mathbb{T}^{S_{+}} \times\left[0, \varepsilon_{0}\right], \mathcal{B}_{2}\left(H_{\perp}^{\sigma_{N}}\left(\mathbb{T}_{1}\right)\right.\right.$, $\left.\mathbb{R}^{S_{+}}\right)$) and

$$
\mathcal{S}_{1}^{\perp}(\theta, y)[w] \in \mathcal{O S}_{w}^{2}(N-1), \quad \mathcal{S}_{2}^{\perp}(\theta)[w, w] \in \mathcal{O S}_{w w}^{2}(N-5) .
$$

We then consider the time one flow map $\Phi_{\mathcal{S}}$, associated to the vector field $\mathcal{S}$, and compute the pullback $X_{6}:=\Phi_{\mathcal{S}}^{*} X_{5}=\left(X_{6}^{(\theta)}, X_{6}^{(y)}, X_{6}^{\perp}\right)$ of the vector field $X_{5}$ by $\Phi_{\mathcal{S}}$. By Lemmata 3.17, 3.18 and in view of Remark (3.3), $X_{6}$ is of the form

$$
\begin{aligned}
X_{6}^{(\theta)}(\mathfrak{x})= & -\omega-\varepsilon \widehat{\omega}-\nabla_{y} Q(y)+\omega \cdot \partial_{\theta} \mathcal{S}^{(\theta)}(\theta)[w, w]-\mathcal{S}^{(\theta)}(\theta)\left[\mathrm{i} \Omega_{\perp} w, w\right] \\
& -\mathcal{S}^{(\theta)}(\theta)\left[w, \mathrm{i} \Omega_{\perp} w\right]-\Upsilon_{2}^{(\theta)}(\theta)[w, w]+\mathcal{O}_{3}^{(\theta)}(\mathfrak{x}) \\
X_{6}^{(y)}(\mathfrak{x})= & \mathcal{O}_{3}^{(y)}(\mathfrak{x}), \\
X_{6}^{\perp}(\mathfrak{x})= & \mathrm{i} \Omega_{\perp} w+\mathcal{D}_{5}^{\perp}(\mathfrak{x})[w]+\left(\omega \cdot \partial_{\theta} \mathcal{S}_{1}^{\perp}(\theta, y)+\left[\mathrm{i} \Omega_{\perp}, \mathcal{S}_{1}^{\perp}(\theta, y)\right]_{l i n}+\mathcal{R}_{N, 1}^{\perp}(\theta, y)\right)[w] \\
+ & \omega \cdot \partial_{\theta} \mathcal{S}_{2}^{\perp}(\theta)[w, w]+\mathrm{i} \Omega_{\perp} \mathcal{S}_{2}^{\perp}(\theta)[w, w]-\mathcal{S}_{2}^{\perp}(\theta)\left[\mathrm{i} \Omega_{\perp} w, w\right] \\
- & \mathcal{S}_{2}^{\perp}(\theta)\left[w, \mathrm{i} \Omega_{\perp} w\right]+\mathcal{R}_{N, 2}^{\perp}(\theta)[w, w]+\mathcal{O B}^{3}(1, N)+\mathcal{O S}^{3}(N-6)
\end{aligned}
$$

where $\mathcal{O}_{3}^{(\theta)}, \mathcal{O}_{3}^{(y)}$ denote terms which are small of order three. The components $\mathcal{S}^{(\theta)}$ and $\mathcal{S}_{1}^{\perp}, \mathcal{S}_{2}^{\perp}$ are now chosen as the solutions of the following homological equations,

$$
\begin{aligned}
\omega \cdot \partial_{\theta} \mathcal{S}^{(\theta)}(\theta)[w, w]-\mathcal{S}^{(\theta)}(\theta)\left[\mathrm{i} \Omega_{\perp} w, w\right]-\mathcal{S}^{(\theta)}(\theta)\left[w, \mathrm{i} \Omega_{\perp} w\right] \\
-\Upsilon_{2}^{(\theta)}(\theta)[w, w]=-\mathcal{Z}^{(\theta)}[w, w], \\
\mathcal{Z}^{(\theta)}[w, w]:=\sum_{j \in S^{\perp}} w_{j} w_{-j}\left\langle\Upsilon_{2}^{(\theta)}(\theta)\left[e^{\mathrm{i} 2 \pi j x}, e^{-\mathrm{i} 2 \pi j x}\right]\right\rangle_{\theta}, \\
\omega \cdot \partial_{\theta} \mathcal{S}_{1}^{\perp}(\theta, y)+\left[\mathrm{i} \Omega_{\perp}, \mathcal{S}_{1}^{\perp}(\theta, y)\right]_{l i n}+\mathcal{R}_{N, 1}^{\perp}(\theta, y)=\mathcal{Z}^{\perp}(y), \\
\quad \mathcal{Z}^{\perp}(y):=\operatorname{diag}_{j \in S^{\perp}}\left[\widehat{\mathcal{R}}_{N, 1}^{\perp}(0, y)\right]_{j}^{j}, \\
\omega \cdot \partial_{\theta} \mathcal{S}_{2}^{\perp}(\theta)[w, w]+\mathrm{i} \Omega_{\perp} \mathcal{S}_{2}^{\perp}(\theta)[w, w]-\mathcal{S}_{2}^{\perp}(\theta)\left[\mathrm{i} \Omega_{\perp} w, w\right] \\
-\mathcal{S}_{2}^{\perp}(\theta)\left[w, \mathrm{i} \Omega_{\perp} w\right]+\mathcal{R}_{N, 2}^{\perp}(\theta)[w, w]=0 .
\end{aligned}
$$

Homological equations of this form can be solved by applying the following lemma.

Lemma 6.1. Let $N \in \mathbb{N}$. (i) Let $\mathcal{M}^{(\theta)} \in C^{\infty}\left(\mathbb{T}^{S_{+}} \times\left[0, \varepsilon_{0}\right], \mathcal{B}_{2}\left(H_{\perp}^{\sigma}\left(\mathbb{T}_{1}\right), \mathbb{R}^{S_{+}}\right)\right)$for some $\sigma>0$ and assume that $\omega \in \Pi_{\gamma}, 0<\gamma<1$ (cf. (1.20)). Then there exists $\mathcal{S}^{(\theta)} \in C^{\infty}\left(\mathbb{T}^{S_{+}} \times\left[0, \varepsilon_{0}\right], \mathcal{B}_{2}\left(H_{\perp}^{\sigma+1}\left(T_{1}\right), \mathbb{R}^{S_{+}}\right)\right)$solving

$$
\begin{aligned}
& \omega \cdot \partial_{\theta} \mathcal{S}^{(\theta)}(\theta)[w, w]-\mathcal{S}^{(\theta)}(\theta)\left[\mathrm{i} \Omega_{\perp} w, w\right]-\mathcal{S}^{(\theta)}(\theta)\left[w, \mathrm{i} \Omega_{\perp} w\right] \\
& \quad-\mathcal{M}^{(\theta)}(\theta)[w, w]=-\mathcal{Z}^{(\theta)}[w, w], \\
& \mathcal{Z}^{(\theta)}[w, w]:=\sum_{j \in S^{\perp}} w_{j} w_{-j}\left\langle\mathcal{M}^{(\theta)}(\theta)\left[e^{\mathrm{i} 2 \pi j x}, e^{-\mathrm{i} 2 \pi j x}\right]\right\rangle_{\theta} .
\end{aligned}
$$


(ii) Let $\mathcal{R}_{N, 1}^{\perp}(\theta, y)[w] \in \mathcal{O S}_{w}^{2}(N)$ and $\omega \in \Pi_{\gamma}, 0<\gamma<1$ (cf. (1.20)). Then there exists $\mathcal{S}_{1}^{\perp}(\theta, y)[w] \in \mathcal{O S}_{w}^{2}(N-1)$ which solves the equation

$$
\begin{gathered}
\omega \cdot \partial_{\theta} \mathcal{S}_{1}^{\perp}(\theta, y)+\left[\mathrm{i} \Omega_{\perp}, \mathcal{S}_{1}^{\perp}(\theta, y)\right]_{l i n}+\mathcal{R}_{N, 1}^{\perp}(\theta, y)=\mathcal{Z}^{\perp}(y), \\
\mathcal{Z}^{\perp}(y):=\operatorname{diag}_{j \in S^{\perp}}\left[\widehat{\mathcal{R}}_{N, 1}^{\perp}(0, y)\right]_{j}^{j} .
\end{gathered}
$$

(iii) Let $\mathcal{R}_{N, 2}^{\perp}(\theta)[w, w] \in \mathcal{O S}_{w w}^{2}(N)$ and assume that $\omega \in \Pi_{\gamma}, 0<\gamma<1(c f .(1.20))$. Then there exists $\mathcal{S}_{2}^{\perp}(\theta)[w, w] \in \mathcal{O S}_{w w}^{2}(N-5)$ which solves the equation

$$
\begin{gathered}
\omega \cdot \partial_{\theta} \mathcal{S}_{2}^{\perp}(\theta)[w, w]+\mathrm{i} \Omega_{\perp} \mathcal{S}_{2}^{\perp}(\theta)[w, w]-\mathcal{S}_{2}^{\perp}(\theta)\left[\mathrm{i} \Omega_{\perp} w, w\right] \\
-\mathcal{S}_{2}^{\perp}(\theta)\left[w, \mathrm{i} \Omega_{\perp} w\right]+\mathcal{R}_{N, 2}^{\perp}(\theta)[w, w]=0 .
\end{gathered}
$$

Proof. Since items (i), ( $i$ i) can be proved by arguments similar to the ones used in the proof of item (iii), we only prove the latter. By assumption, $\mathcal{R}_{N, 2}^{\perp}(\theta, w)[w] \equiv$ $\mathcal{R}_{N, 2}^{\perp}(\theta)[w, w] \in \mathcal{O S}_{w w}^{2}(N)$. Hence there exists an integer $s_{N}>0$ with the property that for any $s \geq s_{N}$, there exists $0<\varepsilon_{0} \equiv \varepsilon_{0}(s)<1$ so that

$$
\begin{gathered}
\mathcal{R}_{N, 2}^{\perp}: \mathbb{T}^{S_{+}} \times\left[0, \varepsilon_{0}\right] \rightarrow \mathcal{B}_{2, s, N},(\theta, \varepsilon) \mapsto \mathcal{R}_{N, 2}^{\perp}(\theta) \equiv \mathcal{R}_{N, 2}^{\perp}(\theta, \varepsilon), \\
\mathcal{B}_{2, s, N}:=\mathcal{B}_{2}\left(H^{s}\left(\mathbb{T}_{1}\right), H^{s+N+1}\left(\mathbb{T}_{1}\right)\right),
\end{gathered}
$$

is $C^{\infty}$-smooth and bounded (cf. (1.39), Defintion 3.4). A a consequence, for any multiindex $\alpha \in \mathbb{Z}_{\geq 0}^{S_{+}}$,

$$
\left\|\partial_{\theta}^{\alpha} \mathcal{R}_{N, 2}^{\perp}(\theta)\right\|_{\mathcal{B}_{2, s, N}} \lesssim \alpha, s 1
$$

Expanding $\mathcal{R}_{N, 2}^{\perp}(\theta)$ in its Fourier series, $\mathcal{R}_{N, 2}^{\perp}(\theta)=\sum_{\ell \in \mathbb{Z}^{S_{+}}} \widehat{\mathcal{R}_{N, 2}^{\perp}}(\ell) e^{\mathrm{i} \ell \cdot \theta}$, the latter estimates imply

$$
\left\|\widehat{\mathcal{R}_{N, 2}^{\perp}}(\ell)\right\|_{\mathcal{B}_{2, s, N}} \lesssim_{\alpha, s}\langle\ell\rangle^{-|\alpha|}, \quad \forall \alpha \in \mathbb{Z}_{\geq 0}^{S_{+}}, \quad \forall \ell \in \mathbb{Z}^{S_{+}}
$$

Since for any $\ell \in \mathbb{Z}^{S_{+}}, \widehat{\mathcal{R}_{N, 2}^{\perp}}(\ell) \in \mathcal{B}_{2, s, N}$, one has for any $w, v \in H_{\perp}^{s}\left(\mathbb{T}_{1}\right)$

$$
\widehat{\mathcal{R}_{N, 2}^{\perp}}(\ell)[w, v]=\sum_{j, j^{\prime} \in S^{\perp}} w_{j} v_{j^{\prime}} \widehat{\mathcal{R}_{N, 2}^{\perp}}(\ell)_{j j^{\prime}}, \quad \widehat{\mathcal{R}_{N, 2}^{\perp}}(\ell)_{j j^{\prime}}(x):=\widehat{\mathcal{R}_{N, 2}^{\perp}}(\ell)\left[e^{\mathrm{i} 2 \pi j x}, e^{\mathrm{i} 2 \pi j^{\prime} x}\right] .
$$

In particular, for $w=e^{\mathrm{i} 2 \pi j x}, v=e^{\mathrm{i} 2 \pi j^{\prime} x}$, one infers from (6.43) that

$$
\left\|\widehat{\mathcal{R}_{N, 2}^{\perp}}(\ell)_{j j^{\prime}}\right\|_{s+N+1} \lesssim \alpha, s\langle\rangle^{-|\alpha|}\langle j\rangle^{s}\left\langle j^{\prime}\right\rangle^{s}, \quad \forall \alpha \in \mathbb{Z}_{\geq 0}^{S_{+}}, \quad \ell \in \mathbb{Z}^{S_{+}}, j, j^{\prime} \in S^{\perp}
$$

Expanding also $\mathcal{S}_{2}^{\perp}(\theta)$ in its Fourier series, $\mathcal{S}_{2}^{\perp}(\theta)=\sum_{\ell \in \mathbb{Z}^{S+}} \widehat{\mathcal{S}_{2}^{\perp}}(\ell) e^{\mathrm{i} \ell \cdot \theta}$, one has for any $w, v \in H_{\perp}^{s}\left(\mathbb{T}_{1}\right)$,

$$
\widehat{\mathcal{S}_{2}^{\perp}}(\ell)[w, v]=\sum_{j, j^{\prime} \in S^{\perp}} w_{j} v_{j^{\prime}} \widehat{\mathcal{S}_{2}^{\perp}}(\ell)_{j j^{\prime}}, \quad \widehat{\mathcal{S}_{2}^{\perp}}(\ell)_{j j^{\prime}}(x):=\widehat{\mathcal{S}_{2}^{\perp}}(\ell)\left[e^{\mathrm{i} 2 \pi j x}, e^{\mathrm{i} 2 \pi j^{\prime} x}\right] .
$$


By expanding $\widehat{\mathcal{S}_{2}^{\perp}}(\ell)_{j j^{\prime}}(x)$ and $\widehat{\mathcal{R}_{2}^{\perp}}(\ell)_{j j^{\prime}}(x)$ with respect to the variable $x \in \mathbb{T}_{1}$ in Fourier series,

$$
\widehat{\mathcal{S}_{2}^{\perp}}(\ell)_{j j^{\prime}}(x)=\sum_{n \in S^{\perp}} \widehat{\mathcal{S}_{2}^{\perp}}(\ell, n)_{j j^{\prime}} e^{\mathrm{i} 2 \pi n x}, \quad \widehat{\mathcal{R}_{N, 2}^{\perp}}(\ell)_{j j^{\prime}}(x)=\sum_{n \in S^{\perp}} \widehat{\mathcal{R}_{N, 2}^{\perp}}(\ell, n)_{j j^{\prime}} e^{\mathrm{i} 2 \pi n x},
$$

the homological equation (6.41) yields the following equations for the coefficients $\widehat{\mathcal{S}_{2}^{\perp}}(\ell, n)_{j j^{\prime}}$ of $\widehat{\mathcal{S}_{2}^{\perp}}(\ell)$,

$$
\mathrm{i}\left(\omega \cdot \ell+\Omega_{n}-\Omega_{j}-\Omega_{j^{\prime}}\right) \widehat{\mathcal{S}_{2}^{\perp}}(\ell, n)_{j j^{\prime}}+\widehat{\mathcal{R}_{N, 2}^{\perp}}(\ell, n)_{j j^{\prime}}=0 .
$$

Since $\omega \in \Pi_{\gamma}^{(3)}, 0<\gamma<1$ (cf. (1.20)), the latter equations admit solutions. They are given by

$$
\widehat{\mathcal{S}_{2}^{\perp}}(\ell, n)_{j j^{\prime}}=-\frac{\widehat{\mathcal{R}_{N, 2}^{\perp}}(\ell, n)_{j j^{\prime}}}{\mathrm{i}\left(\omega \cdot \ell+\Omega_{n}-\Omega_{j}-\Omega_{j^{\prime}}\right)}, \quad \forall \ell \in \mathbb{Z}^{S_{+}}, n, j, j^{\prime} \in S^{\perp},
$$

and satisfy the estimate $\left|\widehat{\mathcal{S}_{2}^{\perp}}(\ell, n)_{j j^{\prime}}\right| \leq\langle\ell\rangle^{\tau}\langle j\rangle^{2}\left\langle j^{\prime}\right\rangle^{2}\langle n\rangle^{2} \gamma^{-1} \widehat{\mathcal{R}_{N, 2}^{\perp}}(\ell, n)_{j j^{\prime}} \mid$ (cf. (1.20)). By (6.47), one has $\left\|\widehat{\mathcal{S}_{2}^{\perp}}(\ell)_{j j^{\prime}}\right\|_{s+N-1}=\left(\sum_{n \in S^{\perp}}\langle n\rangle^{2(s+N-1)}\left|\widehat{\mathcal{S}_{2}^{\perp}}(\ell, n)_{j j^{\prime}}\right|^{2}\right)^{\frac{1}{2}}$ and hence

$$
\begin{aligned}
\left\|\widehat{\mathcal{S}_{2}^{\perp}}(\ell)_{j j^{\prime}}\right\|_{s+N-1} & \leq\langle\ell\rangle^{\tau}\langle j\rangle^{2}\left\langle j^{\prime}\right\rangle^{2} \gamma^{-1}\left(\sum_{n \in S^{\perp}}\langle n\rangle^{2(s+N-1)}\langle n\rangle^{4}\left|\widehat{\mathcal{R}_{N, 2}^{\perp}}(\ell, n)_{j j^{\prime}}\right|^{2}\right)^{\frac{1}{2}} \\
& =\langle\ell\rangle^{\tau}\langle j\rangle^{2}\left\langle j^{\prime}\right\rangle^{2} \gamma^{-1}\left\|\widehat{\mathcal{R}_{N, 2}^{\perp}}(\ell)_{j j^{\prime}}\right\|_{s+N+1} \stackrel{(6.45)}{\lesssim \alpha \alpha, s}\langle\ell\rangle^{\tau-|\alpha|}\langle j\rangle^{s+2}\left\langle j^{\prime}\right\rangle^{s+2} \gamma^{-1}
\end{aligned}
$$

For any $w, v \in H_{\perp}^{s+3}\left(\mathbb{T}_{1}\right)$, one then obtains by the Cauchy-Schwarz inequality,

$$
\begin{aligned}
\left\|\widehat{\mathcal{S}_{2}^{\perp}}(\ell)[w, v]\right\|_{s+N-1} \leq & \sum_{j, j^{\prime} \in S^{\perp}}\left|w_{j}\right|\left|v_{j^{\prime}}\right|\left\|\widehat{\mathcal{S}_{2}^{\perp}}(\ell)_{j j^{\prime}}\right\|_{s+N-1} \\
& \lesssim^{(6.50)}\langle\ell\rangle^{\tau-|\alpha|} \gamma^{-1} \sum_{j, j^{\prime} \in S^{\perp}}\langle j\rangle^{s+2}\left|w_{j}\right|\left\langle j^{\prime}\right\rangle^{s+2}\left|v_{j^{\prime}}\right| \\
& \lesssim \alpha \alpha,\langle\ell\rangle^{\tau-|\alpha|} \gamma^{-1}\|w\|_{s+3}\|v\|_{s+3} .
\end{aligned}
$$

Writing $s$ for $s+3$, we thus have proved that there exists $s_{N}>0$ (large) so that

$$
\left\|\widehat{\mathcal{S}_{2}^{\perp}}(\ell)\right\|_{\mathcal{B}_{2, s, N-4}} \lesssim \alpha, s\langle\ell\rangle^{\tau-|\alpha|} \gamma^{-1}, \quad \forall \alpha \in \mathbb{Z}_{\geq 0}^{S_{+}}, s \geq s_{N}
$$

implying that $\mathcal{S}_{2}^{\perp} \in C^{\infty}\left(\mathbb{T}^{S_{+}} \times\left[0, \varepsilon_{0}\right], \mathcal{B}_{2, s,(N-5)+1}\right)$ for any $s \geq s_{N}$. Hence $\mathcal{S}_{2}^{\perp}(\theta)[w, w]$ $\in \mathcal{O S}_{w w}^{2}(N-5)$. 
By Lemma 6.1 and in view of (6.37), (6.38), the vector field $X_{6}=\left(X_{6}^{(\theta)}, X_{6}^{(y)}, X_{6}^{\perp}\right)$ takes the form

$$
\begin{aligned}
X_{6}^{(\theta)}(\mathfrak{x})= & -\omega-\varepsilon \widehat{\omega}-\nabla_{y} Q(y)-\mathcal{Z}^{(\theta)}[w, w]+\mathcal{O}_{3}^{(\theta)}(\mathfrak{x}), \quad X_{6}^{(y)}(\mathfrak{x})=\mathcal{O}_{3}^{(y)}(\mathfrak{x}), \\
X_{6}^{\perp}(\mathfrak{x})= & \mathrm{i} \Omega_{\perp} w+\mathcal{D}_{6}^{\perp}(\mathfrak{x})[w]+\mathcal{O} \mathcal{B}^{3}(1, N)+\mathcal{O} \mathcal{S}^{3}(N-6), \quad \mathcal{D}_{6}^{\perp}(\mathfrak{x}):=\mathcal{D}_{5}^{\perp}(\mathfrak{x})+\mathcal{Z}^{\perp}(y), \\
& \mathcal{O}_{3}^{(\theta)}, \mathcal{O}_{3}^{(y)} \in C_{b}^{\infty}\left(\left[0, \varepsilon_{0}\right] \times \mathcal{V}^{\sigma_{N}}(\delta), \mathbb{R}^{S_{+}}\right) \quad \text { terms small of order three }
\end{aligned}
$$

for some $\sigma_{N}>0$. Since by (6.22), $\left[\mathcal{R}_{N, 1}^{\perp}(\theta, y)\right]_{j}^{j} \in \mathrm{i} \mathbb{R}, j \in S^{\perp}$, and $\mathcal{Z}^{\perp}(y)=$ $\operatorname{diag}_{j \in S^{\perp}}\left[\widehat{\mathcal{R}_{1}^{\perp}}(0, y)\right]_{j}^{j}$, the operator $\mathcal{Z}^{\perp}(y)$ is a skew-adjoint Fourier multiplier and hence by (6.35) so is $\mathcal{D} \frac{\perp}{6}(\mathfrak{x})$. We summarize our findings as follows.

Proposition 6.3. For any $N \in \mathbb{Z}_{\geq 6}$, there exists an integer $s_{N}>N$ with the property that for any $s \geq s_{N}$, there exist $0<\bar{\delta} \equiv \delta(s, \gamma, N)<1$ and $0<\varepsilon_{0} \equiv \varepsilon_{0}(s, \gamma, N)<1$ so that the following holds. There exists a map $\Psi^{(3)}$ with inverse $\left(\Psi^{(3)}\right)^{-1}$ (cf. Remark 3.4),

$$
\left(\Psi^{(3)}\right)^{ \pm 1} \in \mathcal{C}_{b}^{\infty}\left(\mathcal{V}^{s}(\delta) \times\left[0, \varepsilon_{0}\right], \mathcal{V}^{s}(2 \delta)\right), \quad \forall s \geq s_{N}, \quad\left(\Psi^{(3)}\right)^{ \pm 1}(\mathfrak{x})-\mathfrak{x} \quad \text { small of order two }
$$

so that the transformed vector field $X_{6}:=\left(\Psi^{(3)}\right)^{*} X_{5}=\left(X_{6}^{(\theta)}, X_{6}^{(y)}, X_{6}^{\perp}\right)$ has the form

$$
\begin{gathered}
X_{6}^{(\theta)}(\mathfrak{x})=-\omega-\varepsilon \widehat{\omega}-\nabla_{y} Q(y)-\mathcal{Z}^{(\theta)}[w, w]+\mathcal{O}_{3}^{(\theta)}(\mathfrak{x}), \quad X_{6}^{(y)}(\mathfrak{x})=\mathcal{O}_{3}^{(y)}(\mathfrak{x}), \\
X_{6}^{\perp}(\mathfrak{x})=\mathrm{i} \Omega_{\perp} w+\mathcal{D}_{6}^{\perp}(\mathfrak{x})[w]+\mathcal{O} \mathcal{B}^{3}(1, N)+\mathcal{O S}^{3}(N-6)
\end{gathered}
$$

where $\mathcal{D}_{6}^{\perp}(\mathfrak{x})$ is a Fourier multiplier of order one given by (6.52) and satisfies $\mathcal{D}_{6}^{\perp}(\mathfrak{x})=$ $-\mathcal{D}_{6}^{\perp}(\mathfrak{x})^{\top}$, where

$$
\begin{aligned}
& \mathcal{Z}^{(\theta)} \in \mathcal{B}_{2}\left(H_{\perp}^{\sigma_{N}}, \mathbb{R}^{S_{+}}\right), \\
& \mathcal{Z}^{(\theta)}[w, w]=\sum_{j \in S^{\perp}} w_{j} w_{-j}\left\langle\Upsilon_{2}^{(\theta)}(\theta)\left[e^{\mathrm{i} 2 \pi j x}, e^{-\mathrm{i} 2 \pi j x}\right]\right\rangle_{\theta}, \\
& \forall w \in H_{\perp}^{\sigma_{N}}\left(\mathbb{T}_{1}\right),
\end{aligned}
$$

for some $\sigma_{N}>0$, and where $\mathcal{O}_{3}^{(\theta)}, \mathcal{O}_{3}^{(y)}$ comprises terms which are small of order three.

\section{Proofs of Theorem 4.2 and Theorem 4.3}

First we prove Theorem 4.3.

Proof of Theorem 4.3.. We apply Propositions 5.1, 6.1, 6.2, 6.3. Choose $N=6$ and define

$$
\Phi:=\Phi \circ \Psi^{(1)} \circ \Psi^{(2)} \circ \Psi^{(3)} .
$$

By (5.1), (6.19), (6.33), (6.53), $\Phi$ satisfies property (4.19). Moreover $X=X_{6}=\Phi^{*} X_{\mathcal{H}}$ is given in (6.54) with $N=6$. Hence by setting

$$
\mathrm{D}^{\perp}:=\mathcal{D}_{6}^{\perp}, \quad \mathrm{N}^{(\theta)}(y, w):=-\nabla_{y} Q(y)-\mathcal{Z}^{(\theta)}[w, w],
$$


one has that $\mathrm{D}^{\perp}, \mathrm{N}^{(\theta)}, \mathcal{O}_{3}^{(\theta)}, \mathcal{O}_{3}^{(y)}$ satisfy the properties stated in (4.21). Since $N=6$, the remainder term $\mathcal{O B}^{3}(1,6)+\mathcal{O S}^{3}(0)$ in the expansion of $X^{\perp}(\mathfrak{x})=X_{6}^{\perp}(\mathfrak{x})$ in $(6.54)$ has the form (cf. Definitions 3.1, 3.3)

$$
\Pi_{\perp} \sum_{k=0}^{7} T_{a_{1-k}(\mathfrak{x})} \partial_{x}^{1-k} w+\mathcal{R}_{0}^{\perp}(\mathfrak{x})
$$

with the following property: there are integers $s_{*}, \sigma>0$ so that for any $s \geq s_{*}$ there exist $0<\delta \equiv \delta(s, \gamma)<1$ and $0<\varepsilon_{0} \equiv \varepsilon_{0}(s, \gamma)<1$ so that

$$
\begin{aligned}
& a_{1-k} \in C_{b}^{\infty}\left(\mathcal{V}^{s+\sigma}(\delta) \times\left[0, \varepsilon_{0}\right], H^{s}\left(\mathbb{T}_{1}\right)\right) \text { small of order two, } \quad \forall 0 \leq k \leq 7, \\
& \mathcal{R}_{0}^{\perp} \in C_{b}^{\infty}\left(\mathcal{V}^{s}(\delta) \times\left[0, \varepsilon_{0}\right], H_{\perp}^{s}\left(\mathbb{T}_{1}\right)\right) \text { small of order three. }
\end{aligned}
$$

We then define

$$
a(\mathfrak{x}):=a_{1}(\mathfrak{x}), \quad \mathcal{R}^{\perp}(\mathfrak{x}):=\Pi_{\perp} \sum_{k=0}^{6} T_{a_{-k}(\mathfrak{x})} \partial_{x}^{-k} w+\mathcal{R}_{0}^{\perp}(\mathfrak{x}) .
$$

One shows that $\mathcal{R}^{\perp} \in C_{b}^{\infty}\left(\mathcal{V}^{s}(\delta) \times\left[0, \varepsilon_{0}\right], H_{\perp}^{s}\left(\mathbb{T}_{1}\right)\right)$ for any $s \geq s_{*}+\sigma$ and that $\mathcal{R}^{\perp}$ is small of order three. Indeed, by (7.2) and the estimate (2.2) (paraproduct), it follows that for any $\mathfrak{x} \in \mathcal{V}^{s}(\delta)$,

$$
\begin{aligned}
\left\|\mathcal{R}^{\perp}(\mathfrak{x})\right\|_{s} & \lesssim s, \gamma \max _{0 \leq k \leq 7}\left\|a_{1-k}(\mathfrak{x})\right\|_{1}\|w\|_{s}+\left(\varepsilon+\|y\|+\|w\|_{s}\right)^{3} \\
& \lesssim_{s, \gamma} \max _{0 \leq k \leq 7}\left\|a_{1-k}(\mathfrak{x})\right\|_{s_{*}}\|w\|_{s}+\left(\varepsilon+\|y\|+\|w\|_{s}\right)^{3} \\
& \lesssim_{s, \gamma}\left(\varepsilon+\|y\|+\|w\|_{s_{*}+\sigma}\right)^{3}+\left(\varepsilon+\|y\|+\|w\|_{s}\right)^{3} .
\end{aligned}
$$

Hence we proved that for any $s \geq s_{*}+\sigma$,

$$
\left\|\mathcal{R}^{\perp}(\mathfrak{x})\right\|_{s} \lesssim s, \gamma\left(\varepsilon+\|y\|+\|w\|_{s}\right)^{3} .
$$

Theorem 4.3 then follows by choosing $\sigma_{*}:=s_{*}+\sigma$.

Let us now turn to the proof of Theorem 4.2. It is based on energy estimates for the solutions of the equation $\partial_{t} \mathfrak{x}=X(\mathfrak{x})$ where $X$ is the vector field provided by Theorem 4.3 (cf. (4.20), (4.21))

$$
\left\{\begin{array}{l}
\partial_{t} \theta(t)=-\omega-\varepsilon \widehat{\omega}+\mathrm{N}^{(\theta)}(y, w)+\mathcal{O}_{3}^{(\theta)}(\mathfrak{x}) \\
\partial_{t} y(t)=\mathcal{O}_{3}^{(y)}(\mathfrak{x}) \\
\partial_{t} w(t)=\mathrm{i} \Omega_{\perp} w+\mathrm{D}^{\perp}(\mathfrak{x})[w]+\Pi_{\perp} T_{a(\mathfrak{x})} \partial_{x} w+\mathcal{R}^{\perp}(\mathfrak{x}) .
\end{array}\right.
$$

Choose $\sigma_{*}>0$ and for any $s \geq \sigma_{*}, 0<\delta \equiv \delta(s, \gamma)<1,0<\varepsilon_{0} \equiv \varepsilon_{0}(s, \gamma) \ll \delta$ as in Theorem 4.3. For any $s \geq \sigma_{*}$ and $0<\varepsilon \leq \varepsilon_{0}(s, \gamma)$ we then consider the Cauchy problem of (7.3) with small initial data $\mathfrak{x}_{0}=\left(\theta_{0}, y_{0}, w_{0}\right) \in \mathbb{T}^{S_{+}} \times \mathbb{R}^{S_{+}} \times H_{\perp}^{s}\left(\mathbb{T}_{1}\right)$,

$$
\left|y_{0}\right|,\left\|w_{0}\right\|_{s} \leq \varepsilon .
$$

Increasing $\sigma_{*}$ and decreasing $\varepsilon_{0}$, if needed, it follows from Proposition C. 1 that for any $s \geq \sigma_{*}$ and $0<\varepsilon \leq \varepsilon_{0}$ there exists $T \equiv T_{\varepsilon, s, \gamma}>0$ so that the Cauchy problem 
of (7.3) for any initial data $\mathfrak{x}_{0}=\left(\theta_{0}, y_{0}, w_{0}\right)$ satisfying (7.4) has a unique solution $t \mapsto \mathfrak{x}(t)=(\theta(t), y(t), w(t))$ with

$$
\begin{aligned}
\theta \in C^{1}\left([-T, T], \mathbb{T}^{S_{+}}\right), \quad y \in C^{1}\left([-T, T], \mathbb{R}^{S_{+}}\right), \\
\\
\quad w \in C^{0}\left([-T, T], H_{\perp}^{S}\left(\mathbb{T}_{1}\right)\right) \cap C^{1}\left([-T, T], H_{\perp}^{s-3}\left(\mathbb{T}_{1}\right)\right) .
\end{aligned}
$$

In addition, by Proposition C. 1 there exists $C_{*} \equiv C_{*}(\gamma)>1$ so that

$$
|y(t)|,\|w(t)\|_{s},|\Theta(t)| \leq C_{*} \varepsilon, \quad \forall t \in[-T, T],
$$

where

$$
\Theta(t):=\theta(t)-\theta_{0}+(\omega+\varepsilon \widehat{\omega}) t-\int_{0}^{t} \mathrm{~N}^{\theta}(y(\tau), w(\tau)) d \tau, \quad t \in[-T, T] .
$$

We now prove that the time $T$ of existence of the solution can be chosen to be of size $\varepsilon^{-2}$.

Proposition 7.1. Let $\sigma_{*}$ and $0<\varepsilon_{0} \equiv \varepsilon_{0}(s, \gamma)<1, s \geq \sigma_{*}$ be given as above. Then for any $s \geq \sigma_{*}$ there exists a constant $C_{* *} \equiv C_{* *}(s, \gamma)>0$ so that for any $0<\varepsilon \leq \varepsilon_{0}$, the time of existence $T$ of the solution $\mathfrak{x}(t)$ can be chosen as $T_{\varepsilon, s, \gamma}:=C_{* *} \varepsilon^{-2}$.

To prove the latter proposition, we first need to make some preliminary considerations. Let $s \geq \sigma_{*}$ and $0<\varepsilon \leq \varepsilon_{0}$. By (4.21), $a$ is small of order two and $\mathcal{R}^{\perp}, \mathcal{O}_{3}^{(\theta)}, \mathcal{O}_{3}^{(y)}$ are small of order three, and by applying the estimates (7.6), one has

$$
\begin{gathered}
\left|\mathcal{O}_{3}^{(\theta)}(\mathfrak{x}(t))\right|,\left|\mathcal{O}_{3}^{(y)}(\mathfrak{x}(t))\right| \lesssim \gamma \varepsilon^{3}, \quad\|a(\mathfrak{x}(t))\|_{\sigma_{*}} \lesssim_{\gamma} \varepsilon^{2}, \\
\left\|\mathcal{R}^{\perp}(\mathfrak{x}(t))\right\|_{s} \lesssim_{s, \gamma} \varepsilon^{3}, \quad \forall t \in[-T, T] .
\end{gathered}
$$

First we prove the following lemma.

Lemma 7.1. Given any $s \geq \sigma_{*}$, there exists a constant $K_{0} \equiv K_{0}(s, \gamma)>0$ (large) so that the solutions (7.5) satisfy

$$
|\Theta(t)| \leq K_{0} T \varepsilon^{3}, \quad|y(t)|,\|w(t)\|_{s} \leq \varepsilon+K_{0} \varepsilon^{3} T, \quad \forall t \in[-T, T] .
$$

As a consequence, for any $T>0$ satisfying $T \leq \frac{1}{K_{0}} \varepsilon^{-2}$, one has

$$
|\Theta(t)| \leq \varepsilon, \quad|y(t)|,\|w(t)\|_{s} \leq 2 \varepsilon, \quad \forall t \in[-T, T] .
$$

Proof of Lemma 7.1.. Let $s \geq \sigma_{*}$. First we prove the claimed estimates for $\Theta(t)$ and $y(t)$. By the definition (7.7) of $\Theta$ and (7.3) (Hamiltonian equations), one has

$$
\Theta(0)=0, \quad \partial_{t} \Theta(t)=\mathcal{O}_{3}^{(\theta)}(\mathfrak{x}(t)),
$$

implying that

$$
\Theta(t)=\int_{0}^{t} \mathcal{O}_{3}^{(\theta)}(\mathfrak{x}(\tau)) d \tau
$$

Moreover by (7.3),

$$
y(t)=y_{0}+\int_{0}^{t} \mathcal{O}_{3}^{(y)}(\mathfrak{x}(\tau)) d \tau
$$


By (7.4) and (7.8), one then concludes that there exists a constant $C_{1} \equiv C_{1}(s, \gamma)>0$ so that

$$
|\Theta(t)| \leq C_{1} T \varepsilon^{3}, \quad|y(t)| \leq \varepsilon+C_{1} T \varepsilon^{3}, \quad \forall t \in[-T, T] .
$$

It remains to estimate the $H^{s}$-norm of $w(t)$. To this end recall that for any $w \in H_{\perp}^{s}\left(\mathbb{T}_{1}\right)$,

$$
\|w\|_{s}=\left(\sum_{j \in S^{\perp}}|j|^{2 s}\left|w_{j}\right|^{2}\right)^{\frac{1}{2}}=\left\|\partial_{x}^{s} w\right\|,
$$

where $\left\|\partial_{x}^{s} w\right\|$ denotes the $L^{2}$-norm of $\partial_{x}^{s} w$. Then

$$
\begin{aligned}
\partial_{t}\left\|\partial_{x}^{s} w(t)\right\|^{2} & =\left\langle\partial_{x}^{s} \partial_{t} w(t), \partial_{x}^{s} w(t)\right\rangle+\left\langle\partial_{x}^{s} w(t), \partial_{x}^{s} \partial_{t} w(t)\right\rangle \\
& \stackrel{(7.3)}{=}\left\langle\partial_{x}^{s}\left(\mathrm{i} \Omega_{\perp} w+\mathrm{D}^{\perp}(\mathfrak{x})[w]+\Pi_{\perp} T_{a(\mathfrak{x})} \partial_{x} w+\mathcal{R}^{\perp}(\mathfrak{x})\right), \partial_{x}^{s} w\right\rangle \\
& +\left\langle\partial_{x}^{s} w, \partial_{x}^{s}\left(\mathrm{i} \Omega_{\perp} w+\mathrm{D}^{\perp}(\mathfrak{x})[w]+\Pi_{\perp} T_{a(\mathfrak{x})} \partial_{x} w+\mathcal{R}^{\perp}(\mathfrak{x})\right)\right\rangle .
\end{aligned}
$$

Since $\Omega_{\perp}$ and $\mathrm{D}^{\perp}(\mathfrak{x})$ are both Fourier multipliers, the linear commutators with the Fourier multiplier $\partial_{x}^{S}$ vanish,

$$
\left[\partial_{x}^{s}, \Omega_{\perp}\right]_{l i n}=0, \quad\left[\partial_{x}^{s}, \mathrm{D}^{\perp}(\mathfrak{x})\right]_{l i n}=0 .
$$

Using in addition that $\mathrm{D}^{\perp}(\mathfrak{x})$ is skew-adjoint (cf. (4.21)) and hence $\left(\mathrm{i} \Omega_{\perp}+\mathrm{D}^{\perp}(\mathfrak{x})\right)^{\top}=$ $-\mathrm{i} \Omega_{\perp}-\mathrm{D}^{\perp}(\mathfrak{x})$, one infers

$$
\begin{aligned}
& \left\langle\partial_{x}^{s}\left(\mathrm{i} \Omega_{\perp} w+\mathrm{D}^{\perp}(\mathfrak{x})[w]\right), \partial_{x}^{s} w\right\rangle+\left\langle\partial_{x}^{s} w, \partial_{x}^{s}\left(\mathrm{i} \Omega_{\perp} w+\mathrm{D}^{\perp}(\mathfrak{x})[w]\right)\right\rangle \\
& \quad=\left\langle\left(\mathrm{i} \Omega_{\perp}+\mathrm{D}^{\perp}(\mathfrak{x})\right) \partial_{x}^{s} w, \partial_{x}^{s} w\right\rangle+\left\langle\partial_{x}^{s} w,\left(\mathrm{i} \Omega_{\perp}+\mathrm{D}^{\perp}(\mathfrak{x})\right) \partial_{x}^{s} w\right\rangle \\
& \quad=\left\langle\left(\mathrm{i} \Omega_{\perp}+\mathrm{D}^{\perp}(\mathfrak{x})\right) \partial_{x}^{s} w, \partial_{x}^{s} w\right\rangle+\left\langle\left(\mathrm{i} \Omega_{\perp}+\mathrm{D}^{\perp}(\mathfrak{x})\right)^{\top} \partial_{x}^{s} w, \partial_{x}^{s} w\right\rangle=0 .
\end{aligned}
$$

\section{Moreover}

$$
\begin{aligned}
& \left\langle\partial_{x}^{s} T_{a(\mathfrak{x})} \partial_{x} w, \partial_{x}^{s} w\right\rangle+\left\langle\partial_{x}^{s} w, \partial_{x}^{s} T_{a(\mathfrak{x})} \partial_{x} w\right\rangle \\
& \quad=\left\langle T_{a(\mathfrak{x})} \partial_{x} \partial_{x}^{s} w, \partial_{x}^{s} w\right\rangle+\left\langle\partial_{x}^{s} w, T_{a(\mathfrak{x})} \partial_{x} \partial_{x}^{s} w\right\rangle+\left\langle\left[\partial_{x}^{s}, T_{a(\mathfrak{x})} \partial_{x}\right] w, \partial_{x}^{s} w\right\rangle+\left\langle\partial_{x}^{s} w,\left[\partial_{x}^{s}, T_{a(\mathfrak{x})} \partial_{x}\right] w\right\rangle \\
& \quad=\left\langle\left(T_{a(\mathfrak{x})} \partial_{x}+\left(T_{a(\mathfrak{x})} \partial_{x}\right)^{\top}\right) \partial_{x}^{s} w, \partial_{x}^{s} w\right\rangle+\left\langle\left[\partial_{x}^{s}, T_{a(\mathfrak{x})} \partial_{x}\right] w, \partial_{x}^{s} w\right\rangle+\left\langle\partial_{x}^{s} w,\left[\partial_{x}^{s}, T_{a(\mathfrak{x})} \partial_{x}\right] w\right\rangle .
\end{aligned}
$$

By increasing $\sigma_{*}$ if needed one gets by Corollary 2.2 (with $N=1, m=1$ )

$$
\left\|\Pi_{\perp} T_{a(\mathfrak{x})} \partial_{x}+\Pi_{\perp}\left(T_{a(\mathfrak{x})} \partial_{x}\right)^{\top}\right\|_{\mathcal{B}\left(L_{\perp}^{2}\right)} \lesssim\|a(\mathfrak{x})\|_{\sigma_{*}} \stackrel{(7.8)}{\lesssim} \varepsilon^{2}
$$

and hence by the Cauchy-Schwarz inequality,

$$
\left|\left\langle\left(T_{a(\mathfrak{x})} \partial_{x}+\left(T_{a(\mathfrak{x})} \partial_{x}\right)^{\top}\right) \partial_{x}^{s} w, \partial_{x}^{s} w\right\rangle\right| \lesssim_{\gamma} \varepsilon^{2}\left\|\partial_{x}^{s} w\right\| \lesssim \gamma \varepsilon^{2}\|w\|_{s}^{2} .
$$

Moreover, arguing as in [9, Lemma A.1], one has

$$
\left\|\left[\partial_{x}^{s}, T_{a(\mathfrak{x})} \partial_{x}\right] w\right\|_{L^{2}} \lesssim s\|a(\mathfrak{x})\|_{2}\|w\|_{s} \stackrel{\sigma_{*} \geq 2,(7.8)}{\lesssim s, \gamma} \varepsilon^{2}\|w\|_{s} .
$$


The latter estimate, together with the Cauchy-Schwarz inequality, imply that

$$
\left|\left\langle\left[\partial_{x}^{s}, T_{a(\mathfrak{x})} \partial_{x}\right] w, \partial_{x}^{s} w\right\rangle+\left\langle\partial_{x}^{s} w,\left[\partial_{x}^{s}, T_{a(\mathfrak{x})} \partial_{x}\right] w\right\rangle\right| \lesssim_{s, \gamma} \varepsilon^{2}\|w\|_{s}^{2} .
$$

Finally, by using the Cauchy-Schwarz inequality once more and the estimate (7.8) for $\mathcal{R}^{\perp}$, one gets

$$
\left|\left\langle\partial_{x}^{s} \mathcal{R}^{\perp}(\mathfrak{x}), \partial_{x}^{s} w\right\rangle+\left\langle\partial_{x}^{s} w, \partial_{x}^{s} \mathcal{R}^{\perp}(\mathfrak{x})\right\rangle\right| \lesssim\left\|\mathcal{R}^{\perp}(\mathfrak{x})\right\|_{s}\|w\|_{s} \lesssim s, \gamma \varepsilon^{3}\|w\|_{s} .
$$

Thus, collecting (7.12)-(7.17), and since by (7.6), $\|w(t)\|_{s} \leq C_{*} \varepsilon$ for any $t \in[-T, T]$, one gets

$$
\left|\partial_{t}\left\|\partial_{x}^{s} w(t)\right\|^{2}\right| \lesssim_{s, \gamma} \varepsilon^{4}, \quad \forall t \in[-T, T] .
$$

We then conclude that there exists a constant $C_{2} \equiv C_{2}(s, \gamma)>0$ so that

$$
\|w(t)\|_{s} \leq\left(\left\|w_{0}\right\|_{s}^{2}+C_{2} T \varepsilon^{4}\right)^{1 / 2} \leq \varepsilon\left(1+C_{2} T \varepsilon^{2}\right)^{1 / 2} \leq \varepsilon+C_{2} T \varepsilon^{3}, \quad \forall t \in[-T, T] .
$$

The claimed statement then follows with $K_{0}(s, \gamma):=\max \left\{C_{1}(s, \gamma), C_{2}(s, \gamma)\right\}$.

Proof of Proposition 7.1.. For any given $s \geq \sigma_{*}, 0<\varepsilon \leq \varepsilon_{0}$, and initial data satisfying (7.4), consider the solution $t \mapsto \mathfrak{x}(t)$ in (7.5) of (7.3). It satisfies the estimates (7.9)(7.10) of Lemma 7.1. Let

$$
\check{T}:=\sup \left\{0<T<\frac{1}{K_{0}} \varepsilon^{-2}: 2|\Theta(t)|,|y(t)|,\|w(t)\|_{s} \leq 2 \varepsilon, \forall t \in[-T, T]\right\},
$$

where $K_{0} \equiv K_{0}(s, \gamma)$ is given by Lemma 7.1, and define

$$
M(T):=\max _{|t| \leq T}\left\{2|\Theta(t)|,|y(t)|,\|w(t)\|_{s}\right\}, \quad T \in[0, \check{T}) .
$$

Assume that $\breve{T} \leq \frac{1}{2} \frac{1}{K_{0}} \varepsilon^{-2}$. By the definition of $\breve{T}$ and Proposition C. 1 it then follows that $\sup _{T<\breve{T}} M(T)=2 \varepsilon$. On the other hand, from Lemma 7.1 one infers that

$$
M(\check{T}) \leq \varepsilon+K_{0} \varepsilon^{3} \breve{T} \leq \varepsilon(1+1 / 2) \leq \frac{3}{2} \varepsilon
$$

Hence we obtained a contradiction and thus conclude that $\breve{T}=O\left(\varepsilon^{-2}\right)$.

Proof of Theorem 4.2.. Let $t \mapsto \mathfrak{x}(t)=(\theta(t), y(t), w(t))$ be a curve satisfying (7.4)(7.6). By Theorem 4.3 (Normal Form Theorem), $\mathfrak{x}(t)=(\theta(t), y(t), w(t))$ is a solution of (7.3) if and only if

$$
\mathfrak{x}^{\prime}(t)=\left(\theta^{\prime}(t), y^{\prime}(t), w^{\prime}(t)\right):=\Phi(\mathfrak{x}(t))
$$

is a solution of (4.17) with initial data $\mathfrak{x}_{0}^{\prime}=\Phi\left(\mathfrak{x}_{0}\right)$.

By (4.19) (properties of the transformation $\Phi$ ), for any $\mathfrak{x}$ in $\mathcal{V}^{s}(\delta)$ with $\mathfrak{x}^{\prime}:=\Phi(\mathfrak{x}) \in$ $\mathcal{V}^{s}(\delta)$ one has $\mathfrak{x}=\Phi^{-1}\left(\mathfrak{x}^{\prime}\right)$ and

$$
\left|y^{\prime}\right|,\left\|w^{\prime}\right\|_{s} \leq C(s, \gamma)\left(\varepsilon+|y|+\|w\|_{s}\right), \quad|y|,\|w\|_{s} \leq C(s, \gamma)\left(\varepsilon+\left|y^{\prime}\right|+\left\|w^{\prime}\right\|_{s}\right)
$$

for some constant $C(s, \gamma)>0$. Hence, if $\mathfrak{x}(t)$ satisfies (7.4)-(7.6), then $\mathfrak{x}_{0}^{\prime} \in \mathbb{T}^{S_{+}} \times$ $\mathbb{R}^{S_{+}} \times H_{\perp}^{s}\left(\mathbb{T}_{1}\right)$ with $\left|y_{0}^{\prime}\right|,\left\|w_{0}^{\prime}\right\|_{s} \leq C(s, \gamma) \varepsilon$ and

$$
\theta^{\prime} \in C^{1}\left([-T, T], \mathbb{T}^{S_{+}}\right), \quad y^{\prime} \in C^{1}\left([-T, T], \mathbb{R}^{S_{+}}\right),
$$




$$
w^{\prime} \in C^{0}\left([-T, T], H_{\perp}^{s}\left(\mathbb{T}_{1}\right)\right) \cap C^{1}\left([-T, T], H_{\perp}^{s-3}\left(\mathbb{T}_{1}\right)\right)
$$

with

$$
\left|y^{\prime}(t)\right|,\left\|w^{\prime}(t)\right\|_{s} \leq 2 C(s, \gamma) \varepsilon, \quad \forall t \in[-T, T] .
$$

By Proposition 7.1, $T$ can be chosen as $T_{\varepsilon, s, \gamma}=O\left(\varepsilon^{-2}\right)$. This proves Theorem 4.2 .

\section{Measure Estimates}

In this section we prove the measure estimate (1.23) of the set $\Pi_{\gamma}$ defined in (1.19), (1.20). More precisely we show the following

Proposition 8.1. There exists $\mathrm{a} \in(0,1)$ so that for any $0 \leq j \leq 3$ and any $0<\gamma<1$, $\left|\Pi \backslash \Pi_{\gamma}^{(j)}\right| \lesssim \gamma^{\mathrm{a}}$

We will concentrate on the proof of the claimed measure estimate of $\Pi_{\gamma}^{(3)}$. The ones of $\Pi_{\gamma}^{(0)}, \Pi_{\gamma}^{(1)}$, and $\Pi_{\gamma}^{(2)}$ can be obtained in a similar way and are in fact a bit easier to prove. Recall that

$$
\begin{aligned}
\Pi_{\gamma}^{(3)}=\{\omega & \in \Pi:\left|\omega \cdot \ell+\Omega_{j_{1}}(\omega)+\Omega_{j_{2}}(\omega)+\Omega_{j_{3}}(\omega)\right| \geq \frac{\gamma}{\langle\ell\rangle^{\tau}\left\langle j_{1}\right\rangle^{2}\left\langle j_{2}\right\rangle^{2}\left\langle j_{3}\right\rangle^{2}}, \\
& \left.\forall\left(\ell, j_{1}, j_{2}, j_{3}\right) \in \mathbb{Z}^{S_{+}} \times S^{\perp} \times S^{\perp} \times S^{\perp} \text { with } j_{k}+j_{m} \neq 0, \quad \forall k, m \in\{1,2,3\}\right\}
\end{aligned}
$$

where for any $j \in S^{\perp}, \Omega_{j}(\omega):=\omega_{j}^{k d v}(\mu(\omega), 0)$. One has

$$
\Pi \backslash \Pi_{\gamma}^{(3)} \subset \bigcup_{\substack{\ell \in \mathbb{Z}^{S_{+}}, j_{1}, j_{2}, j_{3} \in S^{\perp} \\ j_{k}+j_{m} \neq 0, \forall k, m \in\{1,2,3\}}} R_{\ell j_{1} j_{2} j_{3}}(\gamma),
$$

where

$$
R_{\ell_{j_{1} j_{2} j_{3}}}(\gamma)=\left\{\omega \in \Pi:\left|\omega \cdot \ell+\Omega_{j_{1}}(\omega)+\Omega_{j_{2}}(\omega)+\Omega_{j_{3}}(\omega)\right|<\frac{\gamma}{\langle\ell\rangle^{\tau}\left\langle j_{1}\right\rangle^{2}\left\langle j_{2}\right\rangle^{2}\left\langle j_{3}\right\rangle^{2}}\right\} .
$$

First we need to establish the following regularity properties and asymptotics for the normal frequencies $\Omega_{j}(\omega), j \in S^{\perp}$.

Lemma 8.1. The map

$$
\Omega^{*}: \Pi \rightarrow \ell^{\infty}\left(S^{\perp}, \mathbb{R}\right), \omega \mapsto\left(\Omega_{j}^{*}(\omega)\right)_{j \in S^{\perp}}, \quad \Omega_{j}^{*}(\omega):=j\left(\Omega_{j}(\omega)-(2 \pi j)^{3}\right),
$$

is real analytic. Furthermore, uniformly on a complex neighborhood of $\Pi$ in $\mathbb{C}^{S_{+}}$,

$$
\Omega_{j}(\omega)=(2 \pi j)^{3}+O\left(j^{-1}\right) \text { as } j \rightarrow \pm \infty .
$$


Proof. Since by [26, Theorem 1.2 (i)], $\Xi \rightarrow \ell^{\infty}\left(S_{+}^{\perp}, \mathbb{R}\right), I \mapsto\left(\omega_{j}^{k d v}(I, 0)\right)_{j \in S_{+}^{\perp}}$ is real analytic and since by [26, Theorem 1.2 (iii)]

$$
\Xi \rightarrow \ell^{\infty}\left(S_{+}^{\perp}, \mathbb{R}\right), I \mapsto\left(j\left(\omega_{j}^{k d v}(I, 0)-(2 \pi j)^{3}\right)\right)_{j \in S_{+}^{\perp}}
$$

is locally bounded in a complex neighborhood of $\Pi$ in $\mathbb{C}^{S_{+}}$, it follows from $[28$, Theorem A.3] that the latter map is real analytic. Furthermore, by [28, Theorem 15.4], the action to frequency map

$$
\Xi \rightarrow \Pi, I=\left(I_{j}\right)_{j \in S_{+}} \mapsto\left(\omega_{j}^{k d v}(I, 0)\right)_{j \in S_{+}}
$$

is real analytic and by the definition of $\Xi$ and $\Pi$, it is a diffeomorphism. Hence its inverse $\mu: \Pi \rightarrow \Xi, \omega \mapsto \mu(\omega)$ is also a real analytic diffeomorphism. Since for any $\omega \in \Pi$ and $j \in S^{\perp}, \Omega_{j}(\omega)=\omega_{j}^{k d v}(\mu(\omega), 0)$ and $\Omega_{j}(\omega)=-\Omega_{-j}(\omega)$ we altogether have proved that the composition

$$
\Omega^{*}: \Pi \rightarrow \ell^{\infty}\left(S^{+}, \mathbb{R}\right), \omega \mapsto\left(j\left(\omega_{j}^{k d v}(\mu(\omega), 0)-(2 \pi j)^{3}\right)_{j \in S^{\perp}}\right.
$$

is real analytic. Since $\Pi \subset \mathbb{R}^{S_{+}}$is compact, $\Omega^{*}$ is actually bounded on a complex neighborhood of $\Pi$ in $\mathbb{C}^{S_{+}}$and hence the claimed asymptotics hold.

Lemma 8.2. There exist constants $C_{0}>0$ and $C_{1}>0$ so that for any $j_{1}, j_{2}, j_{3} \in S^{\perp}$ and any $\ell \in \mathbb{Z}^{S_{+}}$with $|\ell| \geq C_{1}$

$$
\left|R_{\ell j_{1} j_{2} j_{3}}(\gamma)\right| \leq C_{0} \frac{\gamma}{\langle\ell\rangle^{\tau}\left\langle j_{1}\right\rangle^{2}\left\langle j_{2}\right\rangle^{2}\left\langle j_{3}\right\rangle^{2}} .
$$

Proof. Let $\ell \in \mathbb{Z}^{S_{+}} \backslash\{0\}$. Choose $v \in \mathbb{R}^{S_{+}}$with $v \cdot \ell=0$ and introduce $s \mapsto \omega(s):=$ $s \frac{\ell}{|\ell|}+v$. Then $\ell \cdot \omega(s)=s|\ell|$ and hence for any $j_{1}, j_{2}, j_{3} \in S^{\perp}$ and any $s \in \mathbb{R}$ with $\omega(s) \in \Pi$,

$$
\begin{aligned}
& \varphi(s):=\ell \cdot \omega(s)+\Omega_{j_{1}}(\omega(s))+\Omega_{j_{2}}(\omega(s))+\Omega_{j_{3}}(\omega(s)) \\
& =s|\ell|+\Omega_{j_{1}}(\omega(s))+\Omega_{j_{2}}(\omega(s))+\Omega_{j_{3}}(\omega(s)) .
\end{aligned}
$$

By Lemma 8.1 and Cauchy's theorem there exists $C>0$, independent of $j_{1}, j_{2}, j_{3} \in S^{\perp}$, so that

$$
\left|\frac{d}{d s}\left(\Omega_{j_{1}}(\omega(s))+\Omega_{j_{2}}(\omega(s))+\Omega_{j_{3}}(\omega(s))\right)\right| \leq C .
$$

It then follows that $\left|\varphi^{\prime}(s)\right| \geq 1$ for any $|\ell| \geq C_{1}:=C+1$. This implies the claimed estimate.

Lemma 8.3. There exist constants $C_{0}>0, C_{2}>0$ so that for $j_{1}, j_{2}, j_{3} \in S^{\perp}$ with $\min \left\{\left|j_{1}\right|,\left|j_{2}\right|,\left|j_{3}\right|\right\}$

$\geq C_{2}$ one has

$$
R_{0 j_{1} j_{2} j_{3}}(\gamma)=\emptyset, \quad\left|R_{\ell j_{1} j_{2} j_{3}}(\gamma)\right| \leq C_{0} \frac{\gamma}{\langle\ell\rangle^{\tau}\left\langle j_{1}\right\rangle^{2}\left\langle j_{2}\right\rangle^{2}\left\langle j_{3}\right\rangle^{2}}, \quad \forall \ell \in \mathbb{Z}^{S_{+}} \backslash\{0\}
$$


Proof. First we consider the case $\ell=0$. By the asymptotics (8.2) it follows that for any $j_{1}, j_{2}, j_{3} \in S^{\perp}$,

$$
\left|\Omega_{j_{1}}+\Omega_{j_{2}}+\Omega_{j_{3}}\right| \geq 8 \pi^{3}\left|j_{1}^{3}+j_{2}^{3}+j_{3}^{3}\right|-\frac{C}{\min \left\{\left|j_{1}\right|,\left|j_{2}\right|,\left|j_{3}\right|\right\}}
$$

for some constant $C>0$. By the case $n=3$ of Fermat's Last Theorem (cf. [21])

$$
\left|j_{1}^{3}+j_{2}^{3}+j_{3}^{3}\right| \geq 1 \text {. }
$$

Requesting that $\min \left\{\left|j_{1}\right|,\left|j_{2}\right|,\left|j_{3}\right|\right\} \geq C_{2}:=2 C$, one gets $\left|\Omega_{j_{1}}+\Omega_{j_{2}}+\Omega_{j_{3}}\right| \geq 4 \pi^{3}$ and hence $R_{0 j_{1} j_{2} j_{3}}(\gamma)=\emptyset$ for any such $j_{1}, j_{2}, j_{3}$ in $S^{\perp}$.

Now let us consider the case $\ell \in \mathbb{Z}^{S_{+}} \backslash\{0\}$. For any given $j_{1}, j_{2}, j_{3} \in S^{\perp}$, define $s \mapsto \varphi(s)$ as in the proof of Lemma 8.2,

$$
\varphi(s):=|\ell| s+\Omega_{j_{1}}(\omega(s))+\Omega_{j_{2}}(\omega(s))+\Omega_{j_{3}}(\omega(s)) .
$$

By Lemma 8.1 there exists $C>0$, independent of $j_{1}, j_{2}, j_{3} \in S^{\perp}$, so that

$$
\left|\frac{d}{d s} j_{k} \Omega_{j_{k}}(\omega(s))\right| \leq C, \quad \forall 1 \leq k \leq 3
$$

By increasing $C_{2}$ if needed, it follows that for $j_{1}, j_{2}, j_{3} \in S^{\perp}$ satisfying $\min \left\{\left|j_{1}\right|,\left|j_{2}\right|,\left|j_{3}\right|\right\} \geq$ $C_{2}$,

$$
\left|\varphi^{\prime}(s)\right| \geq|\ell|-\frac{3 C}{\min \left\{\left|j_{1}\right|,\left|j_{2}\right|,\left|j_{3}\right|\right\}} \geq \frac{1}{2} .
$$

This implies the claimed measure estimate (8.3).

Lemma 8.4. There exists a constant $C_{3} \geq \max \left\{C_{2}, C_{1}\right\}$, where $C_{2}$ is the constant of Lemma 8.3 and $C_{1}$ the constant of Lemma 8.2, so that

$$
R_{\ell j_{1} j_{2} j_{3}}(\gamma)=\emptyset \quad \forall \ell \in \mathbb{Z}^{S_{+}} \text {with }|\ell|<C_{1} \text { and } \forall j_{1}, j_{2}, j_{3} \in S^{\perp} \text { satisfying }(*)
$$

where

(*) $\quad j_{k}+j_{m} \neq 0, \quad \forall k, m \in\{1,2,3\}, \quad \min \left\{\left|j_{1}\right|,\left|j_{2}\right|,\left|j_{3}\right|\right\}<C_{2}, \quad \max \left\{\left|j_{1}\right|,\left|j_{2}\right|,\left|j_{3}\right|\right\} \geq C_{3}$.

Proof. Let $\ell \in \mathbb{Z}^{S_{+}}$with $|\ell| \leq C_{1}$ and $j_{1}, j_{2}, j_{3} \in S^{\perp}$ with $\min \left\{\left|j_{1}\right|,\left|j_{2}\right|,\left|j_{3}\right|\right\} \leq C_{2}$ and $j_{k}+j_{m} \neq 0$ for any $k, m \in\{1,2,3\}$. First consider the case where $\left|j_{2}\right|,\left|j_{3}\right|<C_{2}$. By Lemma 8.1 one then has for $\left|j_{1}\right| \geq C_{3}$ with $C_{3}>0$ chosen large enough,

$\left|\omega \cdot \ell+\Omega_{j_{1}}+\Omega_{j_{2}}+\Omega_{j_{3}}\right| \geq 8 \pi^{3}\left(\left|j_{1}\right|^{3}-\left|j_{2}\right|^{3}-\left|j_{3}\right|^{3}\right)-C-|\omega| C_{1} \geq C_{3}^{3}-2 C_{2}^{3}-C-|\omega| C_{1} \geq 1$,

implying that $R_{\ell j_{1} j_{2} j_{3}}(\gamma)=\emptyset$.

Let us now turn to the case where $\left|j_{1}\right|,\left|j_{2}\right| \geq C_{3}$ and $\left|j_{3}\right| \leq C_{2}$. If $j_{1}$ and $j_{2}$ have the same sign, then one concludes again that $\left|\omega \cdot \ell+\Omega_{j_{1}}+\Omega_{j_{2}}+\Omega_{j_{3}}\right| \geq 8 \pi^{3}\left(\left|j_{1}\right|^{3}+\left|j_{2}\right|^{3}-\left|j_{3}\right|^{3}\right)-C-|\omega| C_{1} \geq 2 C_{3}^{3}-C_{2}^{3}-C-|\omega| C_{1} \geq 1$ 
by increasing $C_{3}$ if needed. Hence again $R_{\ell j_{1} j_{2} j_{3}}(\gamma)=\emptyset$. Now assume that $j_{1}$ and $j_{2}$ do not have the same sign. Since by assumption, $j_{1}+j_{2} \neq 0$, one has $\left|j_{1}\right|-\left|j_{2}\right| \neq 0$ and it then follows that

$$
\begin{aligned}
\left|\omega \cdot \ell+\Omega_{j_{1}}+\Omega_{j_{2}}+\Omega_{j_{3}}\right| & \geq\left.|| j_{1}\right|^{3}-\left.\left|j_{2}\right|^{3}|-| j_{3}\right|^{3}-C-C_{1}|\omega| \\
& \geq\left|\left(\left|j_{1}\right|-\left|j_{2}\right|\right)\right|\left(\left|j_{1}\right|^{2}+\left|j_{1}\right|\left|j_{2}\right|+\left|j_{2}\right|^{2}\right)-C_{2}^{3}-C-C_{1}|\omega| \\
& \geq 3 C_{3}^{2}-C_{2}^{3}-C-C_{1}|\omega| \geq 1
\end{aligned}
$$

by increasing $C_{3}$ once more if needed. We conclude that also in this case $R_{\ell_{j_{1} j_{2} j_{3}}}(\gamma)=\emptyset$.

Proof of Proposition 8.1. As already mentioned, we concentrate on the proof of the claimed estimate for $\left|\Pi \backslash \Pi_{\gamma}^{(3)}\right|$. In view of Lemmas 8.2-8.4, it remains to estimate the measure of the finite union

$$
\bigcup_{\substack{|\ell| \leq C_{1} \\\left|j_{1}\right|,\left|j_{2}\right|,\left|j_{3}\right| \leq C_{3}}} R_{\ell j_{1} j_{2} j_{3}}(\gamma)
$$

where $C_{1}>0$ is given by Lemma 8.2 and $C_{3}>0$ by Lemma 8.4. By Lemma 8.1, for any $\ell \in \mathbb{Z}^{S_{+}}, j_{1}, j_{2}, j_{3} \in S^{\perp}$ with $|\ell| \leq C_{1}$ and $\left|j_{1}\right|,\left|j_{2}\right|,\left|j_{3}\right| \leq C_{3}$, the function

$$
\omega \mapsto \omega \cdot \ell+\Omega_{j_{1}}(\omega)+\Omega_{j_{2}}(\omega)+\Omega_{j_{3}}(\omega)
$$

is real analytic and by [28, Proposition 15.5], does not vanish identically. Hence by the Weierstrass Preparation Theorem (cf. [8, Lemma 9.7], [10, Proposition 3.1]), for any given $C>0$ there exists a $\in(0,1)$ so that

$$
\bigcup_{\substack{|\ell| \leq C_{1} \\\left|j_{1}\right|,\left|j_{2}\right|,\left|j_{3}\right| \leq C_{3}}}\left\{\omega \in \Pi:\left|\omega \cdot \ell+\Omega_{j_{1}}(\omega)+\Omega_{j_{2}}(\omega)+\Omega_{j_{3}}(\omega)\right| \leq C \gamma\right\} \mid \lesssim \gamma^{\mathrm{a}}
$$

and the claimed estimate for $\left|\Pi \backslash \Pi_{\gamma}^{(3)}\right|$ follows.

Remark 8.1. Note that there exist (many) non-trivial solutions of the diophantine equation

$$
j_{1}^{3}+j_{2}^{3}+j_{3}^{3}+j_{4}^{3}=0
$$

where $\left(j_{1}, j_{2}, j_{3}, j_{4}\right) \in \mathbb{Z}^{4}$ is said to be a trivial solution if there exist $1 \leq \alpha<\beta \leq 4$ so that $j_{\alpha}=-j_{\beta}$. The following example was suggested by Michela Procesi,

$$
(10)^{3}+9^{3}+(-1)^{3}+(-12)^{3}=0 \text {. }
$$

We therefore expect that Lemma 8.3 does not extend to the sets $R_{\ell_{j_{1} j_{2} j_{3} j_{4}}}(\gamma)$, defined as

$$
R_{\ell j_{1} j_{2} j_{3} j_{4}}(\gamma):=\left\{\omega \in \Pi:\left|\omega \cdot \ell+\sum_{k=1}^{4} \Omega_{j_{k}}(\omega)\right|<\frac{\gamma}{\langle\ell\rangle^{\tau}\left\langle j_{1}\right\rangle^{2}\left\langle j_{2}\right\rangle^{2}\left\langle j_{3}\right\rangle^{2}\left\langle j_{4}\right\rangle^{2}}\right\}
$$


and hence that an estimate for $\left|\Pi \backslash \Pi_{\gamma}^{(4)}\right|$ of the type as in Proposition 8.1 for $\left|\Pi \backslash \Pi_{\gamma}^{(3)}\right|$ does not hold. Here $\Pi_{\gamma}^{(4)}$ is defined as

$$
\begin{aligned}
\Pi_{\gamma}^{(4)}:= & \left\{\omega \in \Pi:\left|\omega \cdot \ell+\sum_{k=1}^{4} \Omega_{j_{k}}(\omega)\right| \geq \frac{\gamma}{\langle\ell\rangle^{\tau}\left\langle j_{1}\right\rangle^{2}\left\langle j_{2}\right\rangle^{2}\left\langle j_{3}\right\rangle^{2}\left\langle j_{4}\right\rangle^{2}}\right. \\
& \left.\forall\left(\ell, j_{1}, j_{2}, j_{3}, j_{4}\right) \in \mathbb{Z}^{S_{+}} \times\left(S^{\perp}\right)^{4} \text { with } j_{k}+j_{m} \neq 0 \quad \forall k, m \in\{1,2,3,4\}\right\} .
\end{aligned}
$$

Acknowledgements. We would like to thank Michela Procesi for the example in Remark 8.1 and very valuable feedback and Massimiliano Berti for insightful discussions.

Funding Open Access funding provided by Universität Zürich.

Open Access This article is licensed under a Creative Commons Attribution 4.0 International License, which permits use, sharing, adaptation, distribution and reproduction in any medium or format, as long as you give appropriate credit to the original author(s) and the source, provide a link to the Creative Commons licence, and indicate if changes were made. The images or other third party material in this article are included in the article's Creative Commons licence, unless indicated otherwise in a credit line to the material. If material is not included in the article's Creative Commons licence and your intended use is not permitted by statutory regulation or exceeds the permitted use, you will need to obtain permission directly from the copyright holder. To view a copy of this licence, visit http://creativecommons.org/licenses/by/4.0/.

Publisher's Note Springer Nature remains neutral with regard to jurisdictional claims in published maps and institutional affiliations.

\section{A. Linear Vector Fields on $H_{\perp}^{s}\left(\mathbb{T}_{1}\right)$}

In this appendix we discuss properties of linear vector fields on $H_{\perp}^{s}\left(\mathbb{T}_{1}\right)$, used throughout the main body of the paper. Let $X$ be an unbounded linear vector field on $H_{\perp}^{s}\left(\mathbb{T}_{1}\right), s \in \mathbb{N}$, with domain $H_{\perp}^{s+1}\left(\mathbb{T}_{1}\right)$,

$$
X: H_{\perp}^{s}\left(\mathbb{T}_{1}\right) \rightarrow H_{\perp}^{s-1}\left(\mathbb{T}_{1}\right),
$$

which admits an expansion of order $N \in \mathbb{N}$,

$$
X[w]=\sum_{k=0}^{N+1} \lambda_{1-k} \partial_{x}^{1-k} w+\mathcal{R}_{N}[w], \quad \lambda_{1-k} \in \mathbb{R}, \quad \forall 0 \leq k \leq N+1,
$$

where the remainder $\mathcal{R}_{N}$ is $(N+1)$-regularizing, $\mathcal{R}_{N} \in \mathcal{B}\left(H_{\perp}^{s}\left(\mathbb{T}_{1}\right), H_{\perp}^{s+N+1}\left(\mathbb{T}_{1}\right)\right)$. If in addition, $X$ is a Hamiltonian linear vector field on $H_{\perp}^{s}\left(\mathbb{T}_{1}\right)$,

$$
X[w]=\partial_{x} \nabla H[w], \quad H(w):=\frac{1}{2} \int_{0}^{1} A[w] \cdot w d x, \quad \forall w \in H_{\perp}^{s}\left(\mathbb{T}_{1}\right),
$$

where $A: H_{\perp}^{s}\left(\mathbb{T}_{1}\right) \rightarrow H_{\perp}^{s}\left(\mathbb{T}_{1}\right)$ is a symmetric, bounded linear operator, then the diagonal matrix elements $X_{j}^{\dot{j}}$ of $X$ satisfy

$$
X_{j}^{j}=\int_{0}^{1} \partial_{x} A\left[e^{\mathrm{i} 2 \pi j x}\right] \cdot e^{-\mathrm{i} 2 \pi j x} d x \in \mathrm{i} \mathbb{R}, \quad \forall j \in S^{\perp} .
$$


Lemma A.1. Let $X$ be a vector field as in (A.1) and assume that its diagonal matrix elements satisfy $X_{j}^{j} \in \mathrm{i} \mathbb{R}$ for any $j \in S^{\perp}$. Then $\lambda_{1-k}=0$ for any $0 \leq k \leq N+1$ with $1-k$ even and $\left(\mathcal{R}_{N}\right)_{j}^{j} \in \mathrm{i} \mathbb{R}$ for any $j \in S^{\perp}$.

Proof. It follows from the assumptions that for any $j \in S^{\perp}$, $X_{j}^{j}=-\bar{X}_{j}^{j}, \quad X_{j}^{j}=\sum_{k=0}^{N+1} \lambda_{1-k}(\mathrm{i} 2 \pi j)^{1-k}+\left(\mathcal{R}_{N}\right)_{j}^{j} \quad$ with $\quad \lambda_{1-k} \in \mathbb{R}, \quad\left(\mathcal{R}_{N}\right)_{j}^{j}=O\left(j^{-N-1}\right)$.

One thus concludes that

$$
\sum_{k=0}^{N+1} \lambda_{1-k}(\mathrm{i} 2 \pi j)^{1-k}+O\left(j^{-N-1}\right)=-\sum_{k=0}^{N+1} \lambda_{1-k}(-1)^{1-k}(\mathrm{i} 2 \pi j)^{1-k}+O\left(j^{-N-1}\right)
$$

and hence $\lambda_{1-k}=0$ for any $0 \leq k \leq N+1$ with $1-k$ even. This implies that

$$
\left(\mathcal{R}_{N}\right)_{j}^{j}=X_{j}^{j}-\sum_{k=0}^{N+1} \lambda_{1-k}(\mathrm{i} 2 \pi j)^{1-k} \in \mathrm{i} \mathbb{R}, \quad \forall j \in S^{\perp}
$$

Consider a vector field $X: H_{\perp}^{s}\left(\mathbb{T}_{1}\right) \rightarrow H_{\perp}^{s-1}\left(\mathbb{T}_{1}\right)$, admitting an expansion of order $N$ of the form

$$
X[w]=\Pi_{\perp} \sum_{k=0}^{N+1} T_{a_{1-k}} \partial_{x}^{1-k} w+\mathcal{R}_{N}[w], \quad a_{1-k} \in H^{s}\left(\mathbb{T}_{1}\right), \quad \forall 0 \leq k \leq N+1
$$

where the remainder $\mathcal{R}_{N}$ is $(N+1)$-regularizing, $\mathcal{R}_{N} \in \mathcal{B}\left(H_{\perp}^{s}\left(\mathbb{T}_{1}\right), H_{\perp}^{s+N+1}\left(\mathbb{T}_{1}\right)\right)$.

Lemma A.2. Let $X$ be a vector field as in (A.3) and assume that $X_{j}^{j} \in \mathrm{i} \mathbb{R}$ for any $j \in S^{\perp}$. Then $\left\langle a_{1-k}\right\rangle_{x}=0$ for any $0 \leq k \leq N+1$ with $1-k$ even and $\left(\mathcal{R}_{N}\right)_{j}^{j} \in \mathrm{i} \mathbb{R}$ for any $j \in S^{\perp}$.

Proof. For any $j \in S^{\perp}$, a direct calculation shows that

$$
X_{j}^{j}=\sum_{k=0}^{N+1} \lambda_{1-k}(\mathrm{i} 2 \pi j)^{1-k}+\left(\mathcal{R}_{N}\right)_{j}^{j}, \quad \lambda_{1-k}:=\left\langle a_{1-k}\right\rangle_{x} \in \mathbb{R}, \quad \forall 0 \leq k \leq N+1
$$

Since by assumption $X_{j}^{j}$ is purely imaginary, the claimed results then follow from Lemma A.1. 


\section{B. Standard Results on Homological Equations}

In this appendix we record two standard results on homological equations, used in our normal form procedure. Without further reference, we use the notations introduced in the paragraph Notations and terminology in Sect. 1.

Lemma B.1. Let $\gamma \in(0,1), \tau>0$, and $\omega \in \mathbb{R}^{S_{+}}$. Assume that

$$
|\omega \cdot \ell| \geq \frac{\gamma}{|\ell|^{\tau}}, \quad \forall \ell \in \mathbb{Z}^{S_{+}} \backslash\{0\},
$$

and that $\mathcal{P} \in C^{\infty}\left(\mathbb{T}^{S_{+}}, B\right)$ where $B$ is a Banach space with norm $\|\cdot\|_{B}$. Then there exists a unique solution $\mathcal{F} \in C^{\infty}\left(\mathbb{T}^{S_{+}}, B\right)$ with zero average of

$$
\omega \cdot \partial_{\theta} \mathcal{F}(\theta)+\mathcal{P}(\theta)=\langle\mathcal{P}\rangle_{\theta}, \quad\langle\mathcal{P}\rangle_{\theta}:=\int_{\mathbb{T}^{S} S_{+}} \mathcal{F}(\theta) d \theta=0 .
$$

It is denoted by $\mathcal{F}(\theta)=-\left(\omega \cdot \partial_{\theta}\right)^{-1}\left(\mathcal{P}(\theta)-\langle\mathcal{P}\rangle_{\theta}\right)$.

Lemma B.2. Let $\Omega_{\perp}: L_{\perp}^{2}\left(\mathbb{T}_{1}\right) \rightarrow L_{\perp}^{2}\left(\mathbb{T}_{1}\right)$ be a (possibly unbounded) Fourier multiplier of diagonal form, $\Omega_{\perp}[w]:=\sum_{n \in S^{\perp}} \Omega_{n} w_{n} e^{\mathrm{i} 2 \pi n x}$, and let $0<\gamma<1, \tau>0$, and $\omega \in \mathbb{R}^{S_{+}}$. Assume that

$$
\left|\omega \cdot \ell+\Omega_{n}\right| \geq \frac{\gamma}{\langle\ell\rangle^{\tau}}, \quad \forall(\ell, n) \in \mathbb{Z}^{S_{+}} \times S^{\perp},
$$

and that $\mathcal{P} \in C^{\infty}\left(\mathbb{T}^{S_{+}}, H_{\perp}^{s}\left(\mathbb{T}_{1}\right)\right)$ for any $s \geq 0$. Then there exists a unique solution $\mathcal{F} \in C^{\infty}\left(\mathbb{T}^{S_{+}}, H_{\perp}^{0}\left(\mathbb{T}_{1}\right)\right)$ of the equation

$$
\left(\omega \cdot \partial_{\theta}+\mathrm{i} \Omega_{\perp}\right) \mathcal{F}(\theta)+\mathcal{P}(\theta)=0 .
$$

Furthermore, $\mathcal{F} \in C^{\infty}\left(\mathbb{T}^{S_{+}}, H_{\perp}^{s}\left(\mathbb{T}_{1}\right)\right)$ for any $s \geq 0$.

\section{A Local Existence Result for $\partial_{t} \mathfrak{x}=X(\mathfrak{x})$}

The goal of this appendix is to state a local existence result for the equation $\partial_{t} \mathfrak{x}=X(\mathfrak{x})$ where $X$ is the vector field, introduced in Theorem 4.3 (cf. (4.20), (4.21)),

$$
\left\{\begin{array}{l}
\partial_{t} \theta=-\omega-\varepsilon \widehat{\omega}+\mathrm{N}^{(\theta)}(y, w)+\mathcal{O}_{3}^{(\theta)}(\mathfrak{x}) \\
\partial_{t} y=\mathcal{O}_{3}^{(y)}(\mathfrak{x}) \\
\partial_{t} w=\mathrm{i} \Omega_{\perp} w+\mathrm{D}^{\perp}(\mathfrak{x})[w]+\Pi_{\perp} T_{a(\mathfrak{x})} \partial_{x} w+\mathcal{R}^{\perp}(\mathfrak{x})
\end{array}\right.
$$

where we assume that the assumptions of Theorem 4.3 are satisfied. In particular, $\omega \in$ $\Pi_{\gamma}, 0<\gamma<1$. This local existence result is used in Sect. 7. It reads as follows.

Proposition C.1. There exists $\sigma_{*}>0$ (large) so that for any integer $s \geq \sigma_{*}$, there exist $0<\varepsilon_{0} \equiv \varepsilon_{0}(s, \gamma)<1$ (small) and $C_{*}=C_{*}(s, \gamma)>1$ (large) with the following property: for any $0<\varepsilon \leq \varepsilon_{0}$, there exists $T=T_{\varepsilon, s, \gamma}>0$ so that for any initial data $\mathfrak{x}_{0}=\left(\theta_{0}, y_{0}, w_{0}\right) \in \mathbb{T}^{S_{+}} \times \mathbb{R}^{S_{+}} \times H_{\perp}^{s}\left(\mathbb{T}_{1}\right)$ with

$$
\left|y_{0}\right| \leq \varepsilon, \quad\left\|w_{0}\right\|_{s} \leq \varepsilon,
$$


there exists a unique solution $\mathfrak{x}(t)=(\theta(t), y(t), w(t)), t \in[-T, T]$, of (C.1) with $\mathfrak{x}(0)=\mathfrak{x}_{0}$ satisfying

$$
\begin{aligned}
& \theta \in C^{1}\left([-T, T], \mathbb{T}^{S_{+}}\right), \quad y \in C^{1}\left([-T, T], \mathbb{R}^{S_{+}}\right), \\
& \quad w \in C^{0}\left([-T, T], H_{\perp}^{s}\left(\mathbb{T}_{1}\right)\right) \cap C^{1}\left([-T, T], H_{\perp}^{s-3}\left(\mathbb{T}_{1}\right)\right) .
\end{aligned}
$$

Furthermore,

$$
|y(t)|,\|w(t)\|_{s},|\Theta(t)| \leq C_{*} \varepsilon \quad \forall t \in[-T, T]
$$

where

$$
\Theta(t):=\theta(t)-\theta_{0}+(\omega+\varepsilon \widehat{\omega}) t-\int_{0}^{t} \mathrm{~N}^{(\theta)}(y(\tau), w(\tau)) d \tau .
$$

The rest of this appendix is devoted to the proof of Proposition C.1, which is based on an iterative scheme. For any given $\mathfrak{x}_{0}$ satisfying (C.2), define inductively a sequence $\mathfrak{x}^{(n)}(t)=\left(\theta^{(n)}(t), y^{(n)}(t), w^{(n)}(t)\right), n \geq 0$, as follows:

$$
\mathfrak{x}^{(0)}(t)=\left(\theta^{(0)}(t), y^{(0)}(t), w^{(0)}(t)\right):=\mathfrak{x}_{0}=\left(\theta_{0}, y_{0}, w_{0}\right)
$$

whereas for $n \geq 1, \mathfrak{x}^{(n)}(t)=\left(\theta^{(n)}(t), y^{(n)}(t), w^{(n)}(t)\right)$ is defined to be the solution (cf. Lemma C.1 below) of

$$
\left\{\begin{array}{l}
\partial_{t} \theta^{(n)}=-\omega-\varepsilon \widehat{\omega}+\mathrm{N}^{(\theta)}\left(y^{(n)}, w^{(n)}\right)+\mathcal{O}_{3}^{(\theta)}\left(\mathfrak{x}^{(n-1)}\right), \\
\partial_{t} y^{(n)}=\mathcal{O}_{3}^{(y)}\left(\mathfrak{x}^{(n-1)}\right) \\
\partial_{t} w^{(n)}=\mathrm{i} \Omega_{\perp} w^{(n)}+\mathrm{D}^{\perp}\left(\mathfrak{x}^{(n-1)}\right)\left[w^{(n)}\right]+\Pi_{\perp} T_{a\left(\mathfrak{x}^{(n-1)}\right)} \partial_{x} w^{(n)}+\mathcal{R}^{\perp}\left(\mathfrak{x}^{(n-1)}\right),
\end{array}\right.
$$

with initial data $\mathfrak{x}^{(n)}(0)=\mathfrak{x}_{0}$. The following lemma holds.

Lemma C.1. There exists $\sigma_{*}>0$ (large) so that for any integer $s \geq \sigma_{*}$, there exist $\varepsilon_{0} \equiv \varepsilon_{0}(s, \gamma)>0($ small $)$ and $C_{*} \equiv C_{*}(s, \gamma)>1$ (large) with the following property: for any $0<\varepsilon \leq \varepsilon_{0}(s, \gamma)$, there exists $T=T_{\varepsilon, s, \gamma}>0$ so that for any initial data $\mathfrak{x}_{0}=\left(\theta_{0}, y_{0}, w_{0}\right) \in \mathbb{T}^{S_{+}} \times \mathbb{R}^{S_{+}} \times H_{\perp}^{S}\left(\mathbb{T}_{1}\right)$ satisfying (C.2) and for any integer $n \geq 0$, the system (C.7) admits a unique solution, satisfying $\theta^{(n)} \in C^{1}\left([-T, T], \mathbb{T}^{S_{+}}\right), y^{(n)} \in$ $C^{1}\left([-T, T], \mathbb{R}^{S_{+}}\right)$, and

$$
w^{(n)} \in C^{0}\left([-T, T], H_{\perp}^{s}\left(\mathbb{T}_{1}\right)\right) \cap C^{1}\left([-T, T], H_{\perp}^{s-3}\left(\mathbb{T}_{1}\right)\right) .
$$

Furthermore,

$$
\left|y^{(n)}(t)\right|,\left\|w^{(n)}(t)\right\|_{s},\left|\Theta^{(n)}(t)\right| \leq C_{*} \varepsilon, \quad \forall t \in[-T, T],
$$

where $\Theta^{(0)}(t):=0$ and

$$
\Theta^{(n)}(t):=\theta^{(n)}(t)-\theta_{0}+(\omega+\varepsilon \widehat{\omega}) t-\int_{0}^{t} \mathrm{~N}^{(\theta)}\left(y^{(n-1)}(\tau), w^{(n-1)}(\tau)\right) d \tau, \quad n \geq 1 .
$$


Proof. We prove the claimed results by induction on $n$. For $n=0$, by the definition (C.6) of $\mathfrak{x}^{(0)}(t)$, the claimed statement holds with $T=1$ and with $\sigma_{*}, \varepsilon_{0}$ given as in Theorem 4.3. Now assume that the claimed statement holds at the step $n \geq 0$ of the induction and let us prove it at the step $n+1$. We first need to make some preliminary considerations. Let $s \geq \sigma_{*}$ and $0<\varepsilon \leq \varepsilon_{0}$. Since by (4.21), $a$ is small of order two and $\mathcal{R}^{\perp}, \mathcal{O}_{3}^{(\theta)}, \mathcal{O}_{3}^{(y)}$ are small of order three, it follows from Theorem 4.3 and the estimates (C.9), which hold by the induction hypothesis, that there exists a constant $C_{s} \equiv C_{s}(\gamma)>0$, independent of $n$, so that for any $t \in[-T, T]$

$$
\begin{gathered}
\left|\mathcal{O}_{3}^{(\theta)}\left(\mathfrak{x}^{(n)}(t)\right)\right|,\left|\mathcal{O}_{3}^{(y)}\left(\mathfrak{x}^{(n)}(t)\right)\right| \leq C_{s} \varepsilon^{3}, \quad\left\|a\left(\mathfrak{x}^{(n)}(t)\right)\right\|_{\sigma_{*}} \leq C_{s} \varepsilon^{2}, \\
\left\|\mathcal{R}^{\perp}\left(\mathfrak{x}^{(n)}(t)\right)\right\|_{s} \leq C_{s} \varepsilon^{3}
\end{gathered}
$$

By the second equation in (C.7), one has

$$
y^{(n+1)}(t)=y_{0}+\int_{0}^{t} \mathcal{O}_{3}^{(y)}\left(\mathfrak{x}^{(n)}(\tau)\right) d \tau,
$$

implying that

$$
y^{(n+1)} \in C^{1}\left([-T, T], \mathbb{R}^{S_{+}}\right), \quad\left|y^{(n+1)}(t)\right| \leq \varepsilon+T C_{s} \varepsilon^{3} \leq C_{*} \varepsilon, \quad \forall t \in[-T, T],
$$

where we have chosen $T>0$ so that $T C_{s} \varepsilon^{2} \leq 1$ small enough. By (C.11) it then also follows that

$$
T\left\|a\left(\mathfrak{x}^{(n)}\right)\right\|_{\sigma_{*}} \leq T C_{S} \varepsilon^{2} \leq 1
$$

To solve the equation for $w^{(n+1)}$ in (C.7), we apply Lemma D.2 in Appendix D with $\mathcal{D}(t)=\mathrm{i} \Omega_{\perp}+\mathrm{D}^{\perp}\left(\mathfrak{x}^{(n)}(t)\right), a=a\left(\mathfrak{x}^{(n)}(t)\right)$, and $f=\mathcal{R}^{\perp}\left(\mathfrak{x}^{(n)}(t)\right)$ to conclude that there exists a unique solution $w^{(n+1)}$ of

$$
\left\{\begin{array}{l}
\partial_{t} w^{(n+1)}=\mathrm{i} \Omega_{\perp} w^{(n+1)}+\mathrm{D}^{\perp}\left(\mathfrak{x}^{(n)}\right)\left[w^{(n+1)}\right]+\Pi_{\perp} T_{a\left(\mathfrak{x}^{(n)}\right)} \partial_{x} w^{(n+1)}+\mathcal{R}^{\perp}\left(\mathfrak{x}^{(n)}\right) \\
w^{(n+1)}(0)=w_{0}
\end{array}\right.
$$

in $C^{0}\left([-T, T], H_{\perp}^{s}\left(\mathbb{T}_{1}\right)\right) \cap C^{1}\left([-T, T], H_{\perp}^{s-3}\left(\mathbb{T}_{1}\right)\right)$ and that $w^{(n+1)}$ satisfies

$$
\left\|w^{(n+1)}(t)\right\|_{s},\left\|\partial_{t} w^{(n+1)}(t)\right\|_{s-3} \lesssim s, \gamma \varepsilon+T\left\|\mathcal{R}^{\perp}\left(\mathfrak{x}^{(n)}\right)\right\|_{s} \stackrel{(\mathrm{C} .11)}{\lesssim s, \gamma} \varepsilon+T C_{s} \varepsilon^{3} \leq C_{*} \varepsilon
$$

since $T C_{s} \varepsilon^{2} \leq 1$. We then define

$$
\Theta^{(n+1)}(t):=\theta^{(n+1)}(t)-\theta_{0}+(\omega+\varepsilon \widehat{\omega}) t-\int_{0}^{t} \mathrm{~N}^{(\theta)}\left(y^{(n+1)}(\tau), w^{(n+1)}(\tau)\right) d \tau . \quad t \in[-T, T] .
$$

By the first equation in (C.7), one gets $\Theta^{(n+1)}(t)=\int_{0}^{t} \mathcal{O}_{3}^{(\theta)}\left(\mathfrak{x}^{(n)}(\tau)\right) d \tau$ and hence, using again (C.11),

$$
\theta^{(n+1)} \in C^{1}\left([-T, T], \mathbb{T}^{S_{+}}\right), \quad\left|\Theta^{(n+1)}(t)\right| \leq C_{*} \varepsilon, \quad \forall t \in[-T, T] .
$$

This concludes the proof of the lemma. 
In order to prove the convergence of the sequence $\left(\mathfrak{x}_{n}(t)\right)_{n \geq 0}$, constructed in Lemma C.1, we prove

Lemma C.2. Under the assumptions of Lemma C.1, for any $n \geq 1$,

$$
\left\|\mathfrak{x}^{(n)}(\cdot)-\mathfrak{x}^{(n-1)}(\cdot)\right\|_{\mathcal{C}_{t}^{0} E_{s-1}},\left\|\partial_{t}\left(\mathfrak{x}^{(n)}(\cdot)-\mathfrak{x}^{(n-1)}(\cdot)\right)\right\|_{\mathcal{C}_{t}^{0} E_{s-4}} \leq 2^{-n}
$$

Proof. By $\left(\right.$ C.7), $\widehat{\mathfrak{x}}^{(n)}(t)=\left(\widehat{\theta}^{(n)}(t), \widehat{y}^{(n)}(t), \widehat{w}^{(n)}(t)\right):=\mathfrak{x}^{(n)}(t)-\mathfrak{x}^{(n-1)}(t)$ satisfies

$$
\left\{\begin{array}{l}
\partial_{t} \widehat{\theta}^{(n)}=f^{(\theta, n)}, \\
\partial_{t} \widehat{y}^{(n)}=f^{(y, n)}, \\
\partial_{t} \widehat{w}^{(n)}=\mathrm{i} \Omega_{\perp} \widehat{w}^{(n)}+\mathrm{D}^{\perp}\left(\mathfrak{x}^{(n)}\right) \widehat{w}^{(n)}+\Pi_{\perp} T_{a(\mathfrak{x}}(n) \partial_{x} \widehat{w}^{(n)}+f^{(\perp, n)},
\end{array}\right.
$$

with $\widehat{\mathfrak{x}}^{(n)}(0)=(0,0,0)$, where

$$
\begin{aligned}
& f^{(\theta, n)}:=\mathrm{N}^{(\theta)}\left(y^{(n)}, w^{(n)}\right)-\mathrm{N}^{(\theta)}\left(y^{(n-1)}, w^{(n-1)}\right)+\mathcal{O}_{3}^{(\theta)}\left(\mathfrak{x}^{(n)}\right)-\mathcal{O}_{3}^{(\theta)}\left(\mathfrak{x}^{(n-1)}\right), \\
& f^{(y, n)}:=\mathcal{O}_{3}^{(y)}\left(\mathfrak{x}^{(n)}\right)-\mathcal{O}_{3}^{(y)}\left(\mathfrak{x}^{(n-1)}\right), \\
& f^{(\perp, n)}:=\left(\mathrm{D}^{\perp}\left(\mathfrak{x}^{(n)}\right)-\mathrm{D}^{\perp}\left(\mathfrak{x}^{(n-1)}\right)\right)\left[w^{(n-1)}\right]+\Pi_{\perp} T_{a\left(\mathfrak{x}^{(n)}\right)-a\left(\mathfrak{x}^{(n-1)}\right)} \partial_{x} w^{(n-1)} \\
&+\mathcal{R}^{\perp}\left(\mathfrak{x}^{(n)}\right)-\mathcal{R}^{\perp}\left(\mathfrak{x}^{(n-1)}\right) .
\end{aligned}
$$

By the properties stated in (4.21) and by the mean value theorem, for some $\sigma>0$ large enough and $s \geq \sigma$, one can show that

$$
\begin{aligned}
& f^{(\theta, n)}, f^{(y, n)} \in C^{0}\left([-T, T], \mathbb{R}^{S_{+}}\right), \quad\left|f^{(\theta, n)}\right| \lesssim\left\|\mathfrak{x}^{(n)}-\mathfrak{x}^{(n-1)}\right\|_{C_{t}^{0} E_{\sigma}} \\
& \quad\left|f^{(y, n)}\right| \lesssim \varepsilon^{2}\left\|\mathfrak{x}^{(n)}-\mathfrak{x}^{(n-1)}\right\|_{C_{t}^{0} E_{\sigma}}, \\
& f^{(\perp, n)} \in C^{0}\left([-T, T], H_{\perp}^{s-1}\left(\mathbb{T}_{1}\right)\right), \quad\left\|f^{(\perp, n)}\right\|_{C_{t}^{0} H_{x}^{s-1}} \lesssim_{s} \varepsilon\left\|\mathfrak{x}^{(n)}-\mathfrak{x}^{(n-1)}\right\|_{C_{t}^{0} E_{s-1}} .
\end{aligned}
$$

Hence we immediately conclude that for any $t \in[-T, T]$,

$$
\left|\widehat{\theta}^{(n)}(t)\right| \lesssim T\left\|\mathfrak{x}^{(n)}-\mathfrak{x}^{(n-1)}\right\|_{C_{t}^{0} E_{\sigma}}, \quad\left|\widehat{y}^{(n)}(t)\right| \lesssim T \varepsilon^{2}\left\|\mathfrak{x}^{(n)}-\mathfrak{x}^{(n-1)}\right\|_{C_{t}^{0} E_{\sigma}} .
$$

Furthermore, by applying Lemma D.2, with $\mathcal{D}(t):=\mathrm{i} \Omega_{\perp}+\mathrm{D}^{\perp}\left(\mathfrak{x}^{(n)}\right), a=a\left(\mathfrak{x}^{(n)}\right)$, $f=f^{(\perp, n)}$, and by the estimate (C.19) for $f^{(\perp, n)}$, one also deduces that

$$
\left\|\widehat{w}^{(n)}(t)\right\|_{s-1} \lesssim s \varepsilon T\left\|\mathfrak{x}^{(n)}-\mathfrak{x}^{(n-1)}\right\|_{\mathcal{C}_{t}^{0} E_{s-1}}, \quad \forall t \in[-T, T]
$$

Therefore, collecting (C.20), (C.21), using the induction hypothesis, and by taking $T$ small enough, one gets $\left\|\mathfrak{x}^{(n+1)}-\mathfrak{x}^{(n)}\right\|_{\mathcal{C}_{t}^{0} E_{s-1}} \leq 2^{-(n+1)}$ which is one of the two claimed estimates at the step $n+1$. The estimate for $\partial_{t}\left(\mathfrak{x}^{(n+1)}-\mathfrak{x}^{(n)}\right)$ can be proved in a similar fashion.

By Lemma C.2 and by a standard telescoping argument, one obtains $\theta^{(n)} \rightarrow \theta, \quad y^{(n)} \rightarrow y, \quad \partial_{t} \theta^{(n)} \rightarrow \partial_{t} \theta, \quad \partial_{t} y^{(n)} \rightarrow \partial_{t} y \quad$ uniformly for $\quad-T \leq t \leq T$.

By the estimates (C.8) and by passing to the limit as $n \rightarrow+\infty$, one then obtains the bounds (C.3) for $\Theta(t)$ and $y(t)$. 
Furthermore,

$$
w^{(n)} \rightarrow w \text { in } C^{0}\left([-T, T], H_{\perp}^{s-1}\right) \cap C^{1}\left([-T, T], H_{\perp}^{s-4}\left(\mathbb{T}_{1}\right)\right) .
$$

and $(\theta(t), y(t), w(t))$ is a smooth solution of (C.1). Furthermore, arguing as at the end of the proof of Lemma D.1, one shows that

$$
w \in C^{0}\left([-T, T], H_{\perp}^{s}\left(\mathbb{T}_{1}\right)\right)
$$

and in turn, using the equation, that $\partial_{t} w \in C^{0}\left([-T, T], H_{\perp}^{s-3}\left(\mathbb{T}_{1}\right)\right)$. One also shows that $w(t)$ satisfies the claimed bound (C.3) by using the bounds on $w^{(n)}$ in (C.9). To prove the uniqueness, take two smooth solutions $\mathfrak{x}_{1}, \mathfrak{x}_{2}$ satisfying the same initial condition $\mathfrak{x}_{1}(0)=\mathfrak{x}_{0}=\mathfrak{x}_{2}(0)$. Then write the equation for the difference $\mathfrak{x}_{1}-\mathfrak{x}_{2}$ and argue as in the proof of Lemma C. 2 to conclude that

$$
\left\|\mathfrak{x}_{1}(t)-\mathfrak{x}_{2}(t)\right\|_{E_{\sigma}} \lesssim \int_{0}^{t}\left\|\mathfrak{x}_{1}(\tau)-\mathfrak{x}_{2}(\tau)\right\|_{E_{\sigma}} d \tau, \quad \forall t \in[-T, T]
$$

for some $\sigma>0$ (large). By the Gronwall Lemma, $\mathfrak{x}_{1}=\mathfrak{x}_{2}$. This concludes the proof of Proposition C.1.

\section{On a Class of Linear Para-Differential Equations}

In this appendix we discuss a well-posedness result for a linear para-differential equation of the form

$$
\partial_{t} w=\mathcal{D}(t)[w]+\Pi_{\perp} T_{a} \partial_{x} w+f, \quad x \in \mathbb{T}_{1}, t \in[-T, T],
$$

in the Sobolev space $H_{\perp}^{s}\left(\mathbb{T}_{1}\right)$ for some integer $s \geq \sigma$ with $\sigma>0$ sufficiently large. Here the linear operator $\mathcal{D}(t)$ is a time-dependent Fourier multiplier of order $m \geq 1$, $\mathcal{D}(t) w(x)=\sum_{n \in S^{\perp}} d_{n}(t) w_{n} e^{\mathrm{i} 2 \pi n x}$ with

$$
\mathcal{D} \in C^{0}\left([-T, T], \mathcal{B}\left(H_{\perp}^{s}\left(\mathbb{T}_{1}\right), H_{\perp}^{s-m}\left(\mathbb{T}_{1}\right)\right)\right), \quad \mathcal{D}(t)=-\mathcal{D}(t)^{\top}, \quad \forall t \in[-T, T],
$$

and the coefficient $a(t, x)$ of the operator $T_{a}$ of para-multiplication by $a$ and the forcing term $f(t, x)$ satisfy

$$
a \in C^{0}\left([-T, T], H_{\perp}^{\sigma}\left(\mathbb{T}_{1}\right)\right), \quad f \in C^{0}\left([-T, T], H_{\perp}^{s}\left(\mathbb{T}_{1}\right)\right) .
$$

The main result of this appendix is Lemma D.2 which is used in the proof of Proposition C.1.

First we consider the initial value problem for Eq. (D.1) with vanishing forcing term,

$$
\partial_{t} w=\mathcal{D}(t)[w]+\Pi_{\perp} T_{a} \partial_{x} w, \quad w(\tau, \cdot)=w_{0}(\cdot),
$$

where the initial time $\tau$ is in $[-T, T]$.

Lemma D.1. There exists $\sigma \geq m$ (large) with the following property: Assume that for some $0<T \leq 1$ and any $s \geq \sigma$, (D.2)-(D.3) hold and $\|a\|_{\mathcal{C}_{t}^{0} H_{x}^{\sigma}} \leq 1$. Then for any $w_{0} \in H_{\perp}^{s}\left(\mathbb{T}_{1}\right)$, there exists a unique solution $w$ of $(\mathrm{D} .4)$ in $C^{0}\left([-T, T], H_{\perp}^{s}\left(\mathbb{T}_{1}\right)\right) \cap$ $C^{1}\left([-T, T], H_{\perp}^{s-m}\left(\mathbb{T}_{1}\right)\right)$. For any $t \in[-T, T]$, it satisfies the estimate

$$
\|w(t)\|_{s},\left\|\partial_{t} w(t)\right\|_{s-m} \lesssim s\left\|w_{0}\right\|_{s} .
$$


Proof. The lemma is proved by constructing a sequence of approximating solutions. To this end we introduce for any integer $N \geq 1$ the finite dimensional subspace $H_{N}$ of $L_{\perp}^{2}\left(\mathbb{T}_{1}\right)$

$$
H_{N}:=\left\{u \in L_{\perp}^{2}\left(\mathbb{T}_{1}\right): u(x)=\sum_{j \in S_{N}^{\perp}} u_{n} e^{\mathrm{i} 2 \pi n x}\right\}, \quad S_{N}^{\perp}:=S^{\perp} \cap[-N, N],
$$

and denote by $\Pi_{N}$ the corresponding $L^{2}$-orthogonal projector $\Pi_{N}: L_{\perp}^{2}\left(\mathbb{T}_{1}\right) \rightarrow H_{N}$. We consider the truncated equation

$$
\partial_{t} w=\Pi_{N}\left(\mathcal{D}(t)[w]+\Pi_{\perp} T_{a} \partial_{x} w\right), \quad w(\tau, \cdot)=\Pi_{N} w_{0},
$$

where $w(t, x)=\sum_{n \in S_{N}^{\perp}} w_{n}(t) e^{\mathrm{i} 2 \pi n x} \in H_{N}$. The equation in (D.7) is a linear nonautonomous ODE on the finite dimensional space $H_{N}$ and hence it admits a unique solution $w^{(N)} \in C^{1}\left([-T, T], H_{N}\right)$. We will show that the sequence $\left(w^{(N)}\right)_{N \geq 1}$ admits a limit, which is the solution of (D.4) with the claimed properties. To this end, in a first step, we prove estimates for the Sobolev norm $\left\|w^{(N)}(t)\right\|_{s}$.

Bound OF $\left\|w^{(N)}(t)\right\|_{s}$. Note that $\left\|w^{(N)}(t)\right\|_{s}=\left\|\partial_{x}^{s} w^{(N)}(t)\right\|$. Since $\mathcal{D}(t)$ is a Fourier multiplier, the commutator $\left[\partial_{x}^{s}, \mathcal{D}(t)\right]$ vanishes and since for any $v \in L_{\perp}^{2}\left(\mathbb{T}_{1}\right)$,

$$
\left\langle\Pi_{\perp} u, v\right\rangle=\langle u, v\rangle, \quad \forall u \in L^{2}\left(\mathbb{T}_{1}\right), \quad\left\langle\Pi_{N} v, g\right\rangle=\langle v, g\rangle, \quad \forall g \in H_{N},
$$

one concludes that

$$
\begin{aligned}
\partial_{t}\left\|\partial_{x}^{s} w^{(N)}\right\|= & \left\langle\partial_{x}^{s}\left(\mathcal{D}(t)\left[w^{(N)}\right]+\Pi_{N} \Pi_{\perp} T_{a} \partial_{x} w^{(N)}\right), \partial_{x}^{s} w^{(N)}\right\rangle \\
& +\left\langle\partial_{x}^{s} w^{(N)}, \partial_{x}^{s}\left(\mathcal{D}(t)\left[w^{(N)}\right]+\Pi_{N} \Pi_{\perp} T_{a} \partial_{x} w^{(N)}\right)\right\rangle \\
= & \left\langle\mathcal{D}(t) \partial_{x}^{s} w^{(N)}, \partial_{x}^{s} w^{(N)}\right\rangle+\left\langle\partial_{x}^{s} w^{(N)}, \mathcal{D}(t) \partial_{x}^{s} w^{(N)}\right\rangle \\
& +\left\langle\partial_{x}^{s} T_{a} \partial_{x} w^{(N)}, \partial_{x}^{s} w^{(N)}\right\rangle+\left\langle\partial_{x}^{s} w^{(N)}, \partial_{x}^{s} T_{a} \partial_{x} w^{(N)}\right\rangle .
\end{aligned}
$$

Analysis of the terms in (D.8). Since by assumption $\mathcal{D}(t)^{\top}=-\mathcal{D}(t)$, one has

$$
\begin{gathered}
\left\langle\mathcal{D}(t) \partial_{x}^{s} w^{(N)}, \partial_{x}^{s} w^{(N)}\right\rangle+\left\langle\partial_{x}^{s} w^{(N)}, \mathcal{D}(t) \partial_{x}^{s} w^{(N)}\right\rangle \\
=\left\langle\left(\mathcal{D}(t)+\mathcal{D}(t)^{\top}\right) \partial_{x}^{s} w^{(N)}, \partial_{x}^{s} w^{(N)}\right\rangle=0 .
\end{gathered}
$$

Analysis of the terms in (D.9). One computes

$$
\begin{aligned}
\left\langle\partial_{x}^{s}\right. & \left.T_{a} \partial_{x} w^{(N)}, \partial_{x}^{s} w^{(N)}\right\rangle+\left\langle\partial_{x}^{s} w^{(N)}, \partial_{x}^{s} T_{a} \partial_{x} w^{(N)}\right\rangle \\
= & \left\langle T_{a} \partial_{x} \partial_{x}^{S} w^{(N)}, \partial_{x}^{s} w^{(N)}\right\rangle+\left\langle\partial_{x}^{s} w^{(N)}, T_{a} \partial_{x} \partial_{x}^{S} w^{(N)}\right\rangle \\
& +\left\langle\left[\partial_{x}^{s}, T_{a} \partial_{x}\right] w^{(N)}, \partial_{x}^{s} w^{(N)}\right\rangle+\left\langle\partial_{x}^{s} w^{(N)},\left[\partial_{x}^{s}, T_{a} \partial_{x}\right] w^{(N)}\right\rangle \\
= & \left\langle\left(T_{a} \partial_{x}+\left(T_{a} \partial_{x}\right)^{\top}\right) \partial_{x}^{s} w^{(N)}, \partial_{x}^{s} w^{(N)}\right\rangle \\
& +\left\langle\left[\partial_{x}^{s}, T_{a} \partial_{x}\right] w^{(N)}, \partial_{x}^{s} w^{(N)}\right\rangle+\left\langle\partial_{x}^{S} w^{(N)},\left[\partial_{x}^{s}, T_{a} \partial_{x}\right] w^{(N)}\right\rangle .
\end{aligned}
$$

By Corollary 2.2 (with $N=1, m=1$ ) there exists an integer $\sigma \geq 1$ so that

$$
\left\|T_{a} \partial_{x}+\left(T_{a} \partial_{x}\right)^{\top}\right\|_{\mathcal{B}\left(L^{2}\right)} \lesssim\|a\|_{\sigma}
$$

and hence by the Cauchy-Schwarz inequality,

$$
\left|\left\langle\left(T_{a} \partial_{x}+\left(T_{a} \partial_{x}\right)^{\top}\right) \partial_{x}^{s} w^{(N)}, \partial_{x}^{s} w^{(N)}\right\rangle\right| \lesssim\|a\|_{\sigma}\left\|\partial_{x}^{s} w^{(N)}\right\|^{2} .
$$


Moreover, arguing as in [9, Lemma A.1], one has

$$
\left\|\left[\partial_{x}^{s}, T_{a} \partial_{x}\right] w^{(N)}\right\| \lesssim s\|a\|_{2}\left\|w^{(N)}\right\|_{s} \stackrel{\sigma \geq 2}{\lesssim}\|a\|_{\sigma}\left\|\partial_{x}^{s} w^{(N)}\right\| .
$$

The latter estimate, together with the Cauchy-Schwarz inequality, imply that

$$
\left|\left\langle\left[\partial_{x}^{s}, T_{a} \partial_{x}\right] w^{(N)}, \partial_{x}^{s} w^{(N)}\right\rangle+\left\langle\partial_{x}^{s} w^{(N)},\left[\partial_{x}^{s}, T_{a} \partial_{x}\right] w^{(N)}\right\rangle\right| \lesssim_{s}\|a\|_{\sigma}\left\|\partial_{x}^{s} w^{(N)}\right\|^{2} .
$$

Using (D.12)-(D.13), one then infers from (D.11)

$$
\left|\left\langle\partial_{x}^{s} T_{a} \partial_{x} w^{(N)}, \partial_{x}^{s} w^{(N)}\right\rangle+\left\langle\partial_{x}^{s} w^{(N)}, \partial_{x}^{s} T_{a} \partial_{x} w^{(N)}\right\rangle\right| \lesssim s\|a\|_{\sigma}\left\|\partial_{x}^{s} w^{(N)}\right\|^{2} .
$$

Combining (D.8), (D.9), (D.10), (D.14), yields the estimate

$$
\left|\partial_{t}\left\|\partial_{x}^{s} w^{(N)}\right\|^{2}\right| \lesssim s\|a\|_{\sigma}\left\|\partial_{x}^{s} w^{(N)}\right\|^{2}
$$

which implies that

$$
\begin{aligned}
\left\|\partial_{x}^{s} w^{(N)}(t)\right\|_{L^{2}}^{2} & \leq\left\|w_{0}\right\|_{s}^{2}+C(s)\left|\int_{\tau}^{t}\left\|a\left(t^{\prime}\right)\right\|_{\sigma}\left\|\partial_{x}^{s} w^{(N)}\left(t^{\prime}\right)\right\|^{2} d t^{\prime}\right| \\
& \leq\left\|w_{0}\right\|_{s}^{2}+C(s)\|a\|_{\mathcal{C}_{t}^{0} H_{x}^{\sigma}}\left|\int_{\tau}^{t}\left\|\partial_{x}^{s} w^{(N)}\left(t^{\prime}\right)\right\|^{2} d t^{\prime}\right|
\end{aligned}
$$

for some constant $C(s)>0$. The Gronwall Lemma (recall that $-T \leq t, \tau \leq T$ ) then implies that

$$
\left\|w^{(N)}(t)\right\|_{s}^{2}=\left\|\partial_{x}^{s} w^{(N)}\right\|^{2} \leq \exp \left(C(s)\|a\|_{\mathcal{C}_{t}^{0} H_{x}^{\sigma}} T\right)\left\|w_{0}\right\|_{s}^{2}, \quad \forall t \in[-T, T] .
$$

Since by assumption $0<T \leq 1$ and $\|a\|_{\mathcal{C}_{t}^{0} H_{x}^{\sigma}} \leq 1$, it then follows that

$$
\left\|w^{(N)}(t)\right\|_{s}^{2}=\left\|\partial_{x}^{S} w^{(N)}\right\|^{2} \leq \exp (C(s))\left\|w_{0}\right\|_{s}^{2}, \quad \forall t \in[-T, T] .
$$

Convergence. Now we pass to the limit $N \rightarrow+\infty$. By (D.17) the sequence of functions $w^{(N)}$ is bounded in $C^{0}\left([-T, T], H_{\perp}^{s}\left(\mathbb{T}_{1}\right)\right) \subseteq L^{\infty}\left([-T, T], H_{\perp}^{s}\left(\mathbb{T}_{1}\right)\right)$ and, up to subsequences,

$$
w^{(N)} \stackrel{w^{*}}{\rightarrow} w \text { in } L^{\infty}\left([-T, T], H_{\perp}^{s}\left(\mathbb{T}_{1}\right)\right), \quad\|w\|_{L_{t}^{\infty} H_{x}^{s}} \leq \liminf _{N \rightarrow+\infty}\left\|w^{(N)}\right\|_{L_{t}^{\infty} H_{x}^{s}}
$$

Claim: $\left(w^{(N)}\right)_{N \geq 1}$ converges to $w$ in $C^{0}\left([-T, T], H_{\perp}^{s}\left(\mathbb{T}_{1}\right)\right) \cap C^{1}\left([-T, T], H_{\perp}^{s-m}\left(\mathbb{T}_{1}\right)\right)$, and $w$ solves (D.4).

We first prove that $w^{(N)}$ is a Cauchy sequence in $C^{0}\left([-T, T], L_{\perp}^{2}\left(\mathbb{T}_{1}\right)\right)$. Indeed, by (D.7), the difference $h^{(N)}:=w^{(N+1)}-w^{(N)}$ solves

$$
\begin{gathered}
\partial_{t} h^{(N)}=\mathcal{D}(t) h^{(N)}+\Pi_{N+1}\left(\Pi_{\perp} T_{a} \partial_{x} h^{(N)}\right)+\left(\Pi_{N+1}-\Pi_{N}\right) \Pi_{\perp} T_{a} \partial_{x} w^{(N)}, \\
h^{(N)}(\tau)=\left(\Pi_{N+1}-\Pi_{N}\right) w_{0},
\end{gathered}
$$

and therefore

$$
\begin{aligned}
\partial_{t}\left\|h^{(N)}(t)\right\|^{2} & =\left\langle\partial_{t} h^{(N)}, h^{(N)}\right\rangle+\left\langle h^{(N)}, \partial_{t} h^{(N)}\right\rangle \\
& \left.=\left\langle\mathcal{D}(t) h^{(N)}, h^{(N)}\right\rangle+\left\langle h^{(N)}, \mathcal{D}(t) h^{(N)}\right\rangle+\left\langle T_{a} \partial_{x} h^{(N)}, h^{(N)}\right\rangle+\left\langle h^{(N)}, T_{a} \partial_{x} h^{(N)}\right)\right\rangle
\end{aligned}
$$




$$
+\left\langle\left(\Pi_{N+1}-\Pi_{N}\right) \Pi_{\perp} T_{a} \partial_{x} w^{(N)}, h^{(N)}\right\rangle+\left\langle h^{(N)},\left(\Pi_{N+1}-\Pi_{N}\right) \Pi_{\perp} T_{a} \partial_{x} w^{(N)}\right\rangle .
$$

Arguing as in (D.10), (D.11)-(D.14), one gets

$$
\begin{aligned}
& \left\langle\mathcal{D}(t) h^{(N)}, h^{(N)}\right\rangle+\left\langle h^{(N)}, \mathcal{D}(t) h^{(N)}\right\rangle=0, \\
& \left|\left\langle T_{a} \partial_{x} h^{(N)}, h^{(N)}\right\rangle+\left\langle h^{(N)}, T_{a} \partial_{x} h^{(N)}\right)\right| \lesssim\|a\|_{\sigma}\left\|h^{(N)}\right\|^{2} .
\end{aligned}
$$

Moreover

$$
\begin{aligned}
& \left|\left\langle\left(\Pi_{N+1}-\Pi_{N}\right) \Pi_{\perp} T_{a} \partial_{x} w^{(N)}, h^{(N)}\right\rangle+\left\langle h^{(N)},\left(\Pi_{N+1}-\Pi_{N}\right) \Pi_{\perp} T_{a} \partial_{x} w^{(N)}\right\rangle\right| \\
& \lesssim\left\|\left(\Pi_{N+1}-\Pi_{N}\right) \Pi_{\perp} T_{a} \partial_{x} w^{(N)}\right\|\left\|h^{(N)}\right\| \lesssim\left\|h^{(N)}\right\|^{2}+\left\|\left(\Pi_{N+1}-\Pi_{N}\right) \Pi_{\perp} T_{a} \partial_{x} w^{(N)}\right\|^{2} \\
& \lesssim\left\|h^{(N)}\right\|^{2}+\left(N^{-2}\left\|T_{a} \partial_{x} w^{(N)}\right\|_{2}\right)^{2} \stackrel{(2.2),(\mathrm{D} .17)}{\lesssim}\left\|h^{(N)}\right\|^{2}+\left(N^{-2}\left\|w_{0}\right\|_{3}\right)^{2} \\
& \quad \stackrel{\sigma \geq 3}{\lesssim}\left\|h^{(N)}\right\|^{2}+\left(N^{-2}\left\|w_{0}\right\|_{\sigma}\right)^{2} .
\end{aligned}
$$

Hence (D.19)-(D.21) imply that

$$
\partial_{t}\left\|h^{(N)}(t)\right\|^{2} \lesssim\left\|h^{(N)}(t)\right\|^{2}+N^{-4}\left\|w_{0}\right\|_{\sigma}^{2}
$$

and, since $\left\|h^{(N)}(\tau)\right\| \leq N^{-2}\left\|w_{0}\right\|_{2}$, we deduce from the Gronwall Lemma that

$$
\left\|w^{(N+1)}-w^{(N)}\right\|_{\mathcal{C}_{t}^{0} L_{x}^{2}} \lesssim N^{-2}\left\|w_{0}\right\|_{\sigma} \exp (C T)^{\frac{1}{2}}
$$

for some constant $C>0$. The above inequality, together with a standard telescoping argument implies that $w^{(N)}$ is a Cauchy sequence in $C^{0}\left([-T, T], L_{\perp}^{2}\left(\mathbb{T}_{1}\right)\right)$. Hence $w^{(N)} \rightarrow \tilde{w} \in C^{0}\left([-T, T], L_{\perp}^{2}\left(\mathbb{T}_{1}\right)\right)$. By (D.18) we have

$$
\tilde{w}=w \in C^{0}\left([-T, T], L_{\perp}^{2}\left(\mathbb{T}_{1}\right)\right) \cap L^{\infty}\left([-T, T], H_{\perp}^{s}\left(\mathbb{T}_{1}\right)\right) .
$$

Next, for any $\bar{s} \in[0, s)$ one has by the interpolation inequality

$$
\left\|w^{(N)}-w\right\|_{L_{t}^{\infty} H_{x}^{\bar{s}}} \leq\left\|w^{(N)}-w\right\|_{L_{t}^{\infty} L_{x}^{2}}^{1-\lambda}\left\|w^{(N)}-w\right\|_{L_{t}^{\infty} H_{x}^{s}}^{\lambda}, \quad \lambda:=\bar{s} / s,
$$

and, since $w^{(N)}$ is bounded in $L^{\infty}\left([-T, T], H_{\perp}^{s}\left(\mathbb{T}_{1}\right)\right)$ (see (D.17)), w $\in L^{\infty}([-T, T]$, $\left.H_{\perp}^{s}\left(\mathbb{T}_{1}\right)\right)$, and $w^{(N)} \rightarrow w \in C^{0}\left([-T, T], L_{\perp}^{\frac{2}{2}}\left(\mathbb{T}_{1}\right)\right)$, we deduce that $w^{(N)} \rightarrow w$ in $C^{\overline{0}}\left([-T, T], H_{\perp}^{\bar{s}}\left(\mathbb{T}_{1}\right)\right)$. Moreover we deduce

$$
\begin{aligned}
\partial_{t} w^{(N)}= & \Pi_{N}\left(\mathcal{D}(t)\left[w^{(N)}\right]+\Pi_{\perp} T_{a} \partial_{x} w^{(N)}\right) \rightarrow \\
& \mathcal{D}(t)[w]+\Pi_{\perp} T_{a} \partial_{x} w \quad \text { in } C^{0}\left([-T, T], H_{\perp}^{\bar{s}-m}\left(\mathbb{T}_{1}\right)\right), \quad \forall \bar{s} \in[0, s) .
\end{aligned}
$$

As a consequence $w \in C^{1}\left([-T, T], H_{\perp}^{\bar{s}-m}\left(\mathbb{T}_{1}\right)\right)$ and $\partial_{t} w=\mathcal{D}(t)[w]+\Pi_{\perp} T_{a} \partial_{x} w$ solves (D.4).

Finally, arguing as in [42], Proposition 5.1.D, it follows that the function $t \rightarrow\|w(t)\|_{s}^{2}$ is Lipschitz. Furthermore, one can show that if $t_{n} \rightarrow t$ then $w\left(t_{n}\right) \rightarrow w(t)$ weakly in $H_{\perp}^{s}\left(\mathbb{T}_{1}\right)$, because $w\left(t_{n}\right) \rightarrow w(t)$ in $H_{\perp}^{\bar{s}}\left(\mathbb{T}_{1}\right)$ for any $\bar{s} \in[0, s)$. As a consequence the sequence $w\left(t_{n}\right) \rightarrow w(t)$ strongly in $H_{\perp}^{s}\left(\mathbb{T}_{1}\right)$. This proves that $w \in C^{0}\left([-T, T], H_{\perp}^{s}\left(\mathbb{T}_{1}\right)\right)$ and therefore $\partial_{t} w \in C^{0}\left([-T, T], H_{\perp}^{s-m}\left(\mathbb{T}_{1}\right)\right)$. 
UNIQUENESS. If $w_{1}, w_{2} \in C^{0}\left([-T, T], H_{\perp}^{s}\left(\mathbb{T}_{1}\right)\right) \cap C^{1}\left([-T, T], H_{\perp}^{s-m}\left(\mathbb{T}_{1}\right)\right), s \geq \sigma$, are solutions of (D.4) with $w_{1}(\tau)=w_{2}(\tau) \in H_{\perp}^{s}\left(\mathbb{T}_{1}\right)$, then $h:=w_{1}-w_{2}$ solves

$$
\partial_{t} h=\mathcal{D}(t) h+\Pi_{\perp} T_{a} \partial_{x} h, \quad h(\tau)=0 .
$$

Arguing as in the proofs of the previous energy estimates, we deduce the energy inequality $\partial_{t}\|h(t)\|^{2} \leq C\|h(t)\|^{2}$. Since $h(\tau)=0$, the Gronwall Lemma implies that $\|h(t)\|^{2}=0$, for any $t \in[-T, T]$. This shows the uniqueness.

The estimate for $\|w\|_{s}$ in (D.5) then follows by (D.17)-(D.18) and the one of $\left\|\partial_{t} w\right\|_{s-m}$ in (D.5) by using the equation.

In the next lemma we consider the inhomogeneous equation (D.1).

Lemma D.2. Let $\sigma \geq m$ and $m$ be given as in Lemma D.1 and assume that for some $0<T \leq 1$ and $s \geq \sigma$, (D.2)-(D.3) hold and $\|a\|_{\mathcal{C}_{t}^{0} H_{x}^{\sigma}} \leq 1$. Then for any $w_{0} \in$ $H_{\perp}^{s}\left(\mathbb{T}_{1}\right)$, there exists a unique solution $t \mapsto w(t)$ of $(\mathrm{D} .1)$ in $C^{0}\left([-T, T], H_{\perp}^{s}\left(\mathbb{T}_{1}\right)\right) \cap$ $C^{1}\left([-T, T], H_{\perp}^{s-m}\left(\mathbb{T}_{1}\right)\right)$, with $w(0)=w_{0}$. For any $t \in[-T, T]$ it satisfies,

$$
\begin{aligned}
& \|w(t)\|_{s} \lesssim_{s}\left\|w_{0}\right\|_{s}+\int_{0}^{t}\|f(\tau)\|_{s} d \tau \lesssim_{s}\left\|w_{0}\right\|_{s}+T\|f\|_{\mathcal{C}_{t}^{0} H_{x}^{s}}, \\
& \left\|\partial_{t} w(t)\right\|_{s-m} \lesssim_{s}\left\|w_{0}\right\|_{s}+T\|f\|_{\mathcal{C}_{t}^{0} H_{x}^{s}}, \quad \forall t \in[-T, T] .
\end{aligned}
$$

Proof. For any $t, \tau \in[-T, T]$, denote by $\Phi(\tau, t)$ the flow map of the para-differential equation (D.4),

$$
\partial_{t} w=\mathcal{D}(t)[w]+\Pi_{\perp} T_{a} \partial_{x} w, \quad w(\tau, \cdot)=w_{0}(\cdot) .
$$

By Lemma D.1, $\Phi(\tau, t)$ is a bounded linear operator $H_{\perp}^{s}\left(\mathbb{T}_{1}\right) \rightarrow H_{\perp}^{s}\left(\mathbb{T}_{1}\right)$ for any $s \geq \sigma$. The estimate (D.5) implies that

$$
\left\|\Phi(\tau, t) w_{0}\right\|_{s} \lesssim_{s}\left\|w_{0}\right\|_{s}, \quad\left\|\partial_{t} \Phi(\tau, t) w_{0}\right\|_{s-m} \lesssim_{s}\left\|w_{0}\right\|_{s} .
$$

The unique solution of the Eq. (D.1) in $C^{0}\left([-T, T], H_{\perp}^{s}\left(\mathbb{T}_{1}\right)\right) \cap C^{1}\left([-T, T], H_{\perp}^{s-m}\left(\mathbb{T}_{1}\right)\right)$ with initial data $w(0)=w_{0}$ is then given by the Duhamel formula $w(t)=\Phi(0, t) w_{0}+$ $\int_{0}^{t} \Phi(\tau, t) f(\tau) d \tau$ and the claimed estimates easily follow.

\section{References}

1. Baldi, P., Berti, M., Montalto, R.: KAM for autonomous quasi-linear perturbations of KdV. Ann. Inst. H. Poincaré Analyse Non Linéaire 33(6), 1589-1638 (2016)

2. Bambusi, D.: Nekhoroshev theoem for small amplitude solutions in nonlinear Schrödinger equations. Math. Z. 130, 345-387 (1999)

3. Bambusi, D.: Birkhoff normal form for some nonlinear PDEs. Commun. Math. Phys. 234(2), 253-285 (2003)

4. Bambusi, D., Grébert, B.: Birkhoff normal form for partial differential equations with tame modulus. Duke Math. J. 135(3), 507-567 (2006)

5. Bernier, J., Faou, E., Grébert, G.: Rational normal forms and stability of small solutions to nonlinear Schrödinger equations, arXiv:1812.11414 (2020)

6. Bernier, J., Grébert, B.: Long time dynamics for generalized Korteweg-de Vries and Benjamin-Ono equations, arXiv:2006.04397 (2020)

7. Berti, M., Delort, J.: Almost global solutions of capillary-gravity water waves equations on the circle. Lecture Notes of the Unione Matematica Italiana, 24, Springer (2018)

8. Berti, M., Kappeler, T., Montalto, R.: Large KAM tori for quasi-linear perturbations of KdV. Arch. Ration. Mech. Anal. 239, 1395-1500 (2021) 
9. Berti, M., Montalto, R.: Quasi-periodic standing wave solutions for gravity-capillary water waves, Memoirs of the Amer. Math. Society, Vol. 263, Number 1273 (2020)

10. Biasco, L., Coglitore, F.: Periodic orbits accumulating onto elliptic tori for the $(N+1)$-body problem. Celest. Mech. Dyn. Astronom. 101(4), 349-373 (2008)

11. Bikbaev, R., Kuksin, S.: On the parametrization of finite-gap solutions by frequency vector and wave number vectors and a theorem by I. Krichever. Lett. Math. Phys. 28, 115-122 (1993)

12. Bourgain, J.: Quasi-periodic solutions of Hamiltonian perturbations of 2D linear Schrödinger equations. Ann. Math. 148, 363-439 (1998)

13. Bourgain, J.: On diffusion in high dimensional Hamiltonian systems and PDE. J. Anal. Math. 80, 1-35 (2000)

14. Boussinesq, J.: Théorie de l'intumescence liquid appelée onde solitaire ou de translation, se propageant dans un canal rectangulaire. C. R. Acad. Sci. (Paris) 72, 755-759 (1871)

15. Chierchia, L., You, J.: KAM tori for 1D nonlinear wave equations with periodic boundary conditions. Commun. Math. Phys. 211(2), 487-525 (2000)

16. Colliander, J., Keel, M., Staffilani, G., Takaoka, H., Tao, T.: Transfer of energy to high frequencies in the cubic defocusing nonlinear Schrödinger equation. Invent. Math. 181(1), 3-113 (2010)

17. Cong, H., Liu, J., Shi, Y., Yuan, X.: The stability of full dimensional KAM tori for nonlinear Schrödinger equation. J. Differ. Equ. 264(7), 4504-4563 (2018)

18. Delort, J.-M.: A quasi-linear Birkhoff normal forms method. Application to the quasi-linear Klein-Gordon equation on $\mathbb{S}^{1}$, Astérisque No. 341 (2012)

19. Dispersive Wiki, http://wiki.math.toronto.edu/DispersiveWiki/

20. Dubrovin, B., Krichever, I., Novikov, S.: Integrable systems I. In: Arnold, V., Novikov, S. (eds.) Dynamical Systems IV, Encyclopedia of Mathematical Sciences vol. 4, pp. 173-280. Springer (1990)

21. Euler, L.: Elements of Algebra, 3rd edn. Longmans, London (1822)

22. Faddev, L., Takhtajan, L.: Hamiltonian Methods in the Theory of Solitons. Springer, Berlin (1987)

23. Feola, R., Iandoli, F.: A non-linear Egorov theorem and Poincaré-Birkhoff normal forms for quasi-linear pdes on the circle, arXiv:2002.12448 (2020)

24. Gardner, C., Greene, J., Kruskal, M., Miura, R.: Korteweg-de Vries equation and generalizations.VI. Methods for exact solution. Commun. Pure Appl. Math. 27, 97-133 (1974)

25. Guardia, M., Hani, Z., Haus, E., Maspero, A., Procesi, M.: A note on growth of Sobolev norms near quasiperiodic finite-gap tori for the 2D cubic NLS equation. Atti Accad. Naz. Lincei Rend. Lincei Mat. Appl. 30(4), 865-880 (2019)

26. Kappeler, T., Molnar, J.: On the wellposedness of the $\mathrm{KdV} / \mathrm{KdV} 2$ equations and their frequency maps. Ann. Inst. H. Poincaré Analyse Non Linéaire 35(1), 101-160 (2018)

27. Kappeler, T., Montalto, R.: Normal form coordinates for the KdV equation having expansions in terms of pseudodifferential operators. Commun. Math. Phys. 375, 833-913 (2020)

28. Kappeler, T., Pöschel, J.: KdV \& KAM. Springer-Verlag, Berlin (2003)

29. Korteweg, D., de Vries, G.: On the change of form of long waves advancing in rectangular canal, and on a new type of long stationary waves. Philos. Mag. Ser. 5 39, 422-443 (1895)

30. Kruskal, M., Zabusky, N.: Interactions of 'solitons' in a collisionless plasma and the recurrence of initial states. Phys. Rev. Lett. 15, 240-243 (1965)

31. Kuksin, S.: Hamiltonian perturbations of infinite-dimensional linear systems with an imaginary spectrum. Funct. Anal. Appl. 21, 192-205 (1987)

32. Kuksin, S.: A KAM theorem for equations of the Korteweg-de Vries type. Rev. Math. Phys. 10(3), 1-64 (1998)

33. Kuksin, S.: Analysis of Hamiltonian PDEs. Oxford University Press, Oxford (2000)

34. Lax, P.: Integrals of of nonlinear equations of evolution and solitary waves. Commun. Pure Appl. Math. 21, 468-490 (1968)

35. Liu, J., Yuan, X.: A KAM theorem for Hamiltonian partial differential equations with unbounded perturbations. Commun. Math. Phys 307(3), 629-673 (2011)

36. Maspero, A., Procesi, M.: Long time stability of small finite gap solutions of the cubic nonlinear Schrödinger equation on $\mathbb{T}^{2}$. J. Differ. Equ. 265(7), 3212-3309 (2018)

37. Métivier, G.: Para-differential calculus and applications to the Cauchy problem for nonlinear systems, Publications of the Scuola Normale Superiore (Book 5), Edizioni della Normale (2008)

38. Miura, R., Gardner, C., Kruskal, M.: Korteweg-de Vries equation and generalizations. II. Existence of conservation laws and constants of motion. J. Math. Phys. 9, 1204-1209 (1968)

39. Procesi, C., Procesi, M.: A KAM algorithm for the resonant non-linear Schrödinger equation. Adv. Math. 272, 399-470 (2015)

40. Rayleigh, L.: On waves. In: Report of the fourteenth Meeting of the British Association for the Association for the Advancement of Sciences, pp. 311-390. John Murray, London (1844) 
41. Wayne, E.: Periodic and quasi-periodic solutions of nonlinear wave equations via KAM theory. Commun. Math. Phys. 127(3), 479-528 (1990)

42. Taylor, M.E.: Pseudo differential operators and nonlinear PDEs, Progress in Mathematics, Vol. 100. Birkhäuser, Boston (1991)

Communicated by C. Liverani 